(C) Copyright 2014

Hugo Solis 



\title{
Axial; \\ a Sound Art Work of an Oceanographic Expedition
}

\author{
Hugo Solis
}

A dissertation

submitted in partial fulfillment of the requirements for the degree of

Doctor of Philosophy

University of Washington

2014

\author{
Reading Committee: \\ Juan Pampin, Chair \\ Richard S. Karpen \\ James Coupe
}

Program Authorized to Offer Degree:

Digital Arts and Experimental Media 



\title{
University of Washington
}

\author{
Abstract \\ Axial; \\ a Sound Art Work of an Oceanographic Expedition \\ Hugo Solis \\ Chair of the Supervisory Committee: \\ Dr. Juan Pampin \\ DXARTS
}

Axial is a sound installation in which a cargo container is employed as a resonant object: resonant in its acoustic sense because the container is used as sound generator, but also resonant in its metaphorical connotation because the container and the generated sounds translate and represent the geological properties of Axial, an active submarine volcano on the Juan de Fuca Ridge located about 250 miles off the coast of Oregon. The container is also the space-instrument for interpreting the scientific data obtained during the oceanographic expedition Enlighten10, during which the artist recorded the sounds of the hydrothermal vents located in the area at depth of over 4,500 feet.

This thesis describes in detail the entire compositional process as well as the concepts, procedures, and context of the artwork. Chapter one offers a general description of the project and should be the starting point for a first-time reader because it helps to establish the context. Chapter two describes the concept of digital plurifocality and presents the properties of the Plurifocal Events Controller, a technological infrastructure for creating spatially distributed sound works. Chapter three describes Juum, a piece of software that helps to organize and create digital artworks using an extended timeline logic employing scripting programming. Chapter four presents the concepts, logic, influences, and preoccupations behind Axial including personal preoccupations about sound art and digital art. Chapter five presents the preparation process required for the creation of the work, explaining the different versions and explorations that were required to achieve the final iteration. Chapter six describes the compositional process of the 

final version as well as the installation and exhibition process. The last chapter presents a set of conclusions that summarizes the experience, the contributions to the field, and the obtained artistic achievements. This chapter relates the work to the artist's current artworks.

A set of five appendixes complement the thesis, offering technical details, links to digital materials and digital scores that help to round the description, and explanations of the realized work which was developed during the doctoral program in Digital Art and Experimental Media at the University of Washington. One of these appendixes presents a basic marine geology context and presents the Ocean Observatory Initiative, an ambitious oceanographic research program that embraced the scientific aspect of Axial.

Resumen Axial es una instalación sonora en donde un contenedor de barco es usado como objeto resonante. Resonante en su sentido acústico es utilizado como generador sonoro, pero también refiere a una connotación metafórica, ya que el contenedor y los sonidos generados en él traducen y representan las propiedades de Axial, un volcán activo submarino localizado en la placa de Juan de Fuca a unas 250 millas de las costas de Óregon. El contenedor también es utilizado como espacio-instrumento para interpretar una serie de datos científicos obtenidos durante la expedición oceanográfica Enlighten10, en la cual el artista grabó los sonidos de las ventilas hidrotermales localizadas en dicha área a unos 4500 pies de profundidad.

Esta tesis describe a detalle el proceso composicional, así como los conceptos, procedimientos y el contexto de la obra artística. El capítulo uno presenta una descripción general de todo el proyecto y debe de tomarse como punto de partida para la lectura inicial, ya que ayuda a establecer el contexto general. El capítulo dos describe los conceptos de la Plurifocalidad Digital así como las propiedades de una tecnología denominada Controlador de Eventos Plurifocales, una infraestructura diseñada para crear obras de sonido distribuido espacialmente. El capítulo tres describe a Juum (un software que ayuda a la creación y organización de trabajos artísticos digitales que extiende la lógica de la línea de tiempo), utilizando programación script. 

El capítulo cuatro presenta los conceptos, lógica, influencia y preocupaciones detrás de Axial, incluyendo pensamientos referidos al arte sonoro y al arte digital. El capítulo cinco presenta el proceso de preparación y describe las diferentes versiones y exploraciones que fueron requeridas para llegar a la versión final. El capítulo seis describe el proceso composicional de la versión final así como su instalación y proceso de exhibición. El último capítulo presenta una serie de conclusiones que resumen la experiencia, las contribuciones al área de estudio y los logros artísticos obtenidos. Este capítulo relaciona la obra con trabajos actuales del artista.

Una serie de cinco apéndices complementan la tesis presentando detalles técnicos, referencias de materiales digitales y partituras digitales que ayudan a redondear la descripción y explicaciones del trabajo realizado durante el doctorado en arte digital y medios experimentales en la University of Washington. Uno de estos apéndices presenta un contexto de geología marina básica y presenta la Ocean Observatory Initiative, una ambiciosa iniciativa de investigación que cobijó los aspectos científicos de este proyecto artístico. 



\section{TABLE OF CONTENTS}

Page

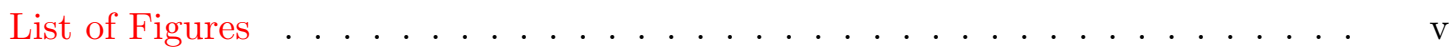

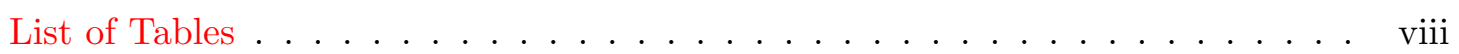

Chapter 1: Introduction . . . . . . . . . . . . . . . 1

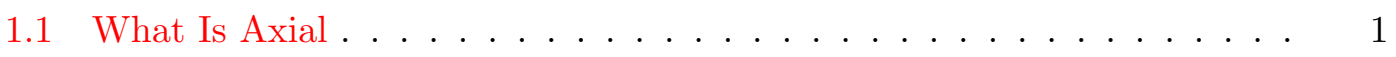

1.2 Organization of the Thesis . . . . . . . . . . . . 4

Chapter 2: Digital Plurifocality and the Plurifocal Events Controller . . . . 7

2.1 Inspirations . . . . . . . . . . . . . . . 7

2.1.1 The Work of Pampin, Karpen, and Coupe . . . . . . . . . . 8

2.1.2 La Ruta del Tambor y el Bombo . . . . . . . . . . . . . . . 9

2.1.3 Ecos del Milenio . . . . . . . . . . . . . . . . . . . . 10

2.1.4 Previous Work by the Author . . . . . . . . . . . . . 11

2.2 Digital Plurifocality . . . . . . . . . . . . . . . . . 11

2.2.1 Physical Space as a Musical Variable . . . . . . . . . . . . 13

2.2.2 Plurifocal Music . . . . . . . . . . . . . . . . . . . . . . 14

2.2.3 Logic of Digital Plurifocality . . . . . . . . . . . . 16

2.3 The Plurifocal Events Controller _. . . . . . . . . . . . . . . 17

2.3.1 Motivations for Creating the Plurifocal Events Controller . . . . 17

2.4 Conclusions . . . . . . . . . . . . . . . . . . . . . . . . 21

Chapter 3: Juum Software . . . . . . . . . . . . . . . . . 22

3.1 Motivation . . . . . . . . . . . . . . . . . 22

3.2 Digital toolkits and similar software . . . . . . . . . . . . 24

3.2 .1 Field . . . . . . . . . . . . . . . . . . . 24

3.2.2 MaxMsp, PureData, VVV, and other graphical software . . . . 25

3.2.3 SuperCollider and other sound-oriented software . . . . . . . . 26

3.2 .4 Software with timelines . . . . . . . . . . . . 26

3.3 Description . . . . . . . . . . . . . . . . . . . 27

3.3 .1 Architecture ........................ 28 
$3.3 .2 \quad$ Modules . . . . . . . . . . . . . . . . . . . . . . . . . 29

3.3.3 Graphical user interface . . . . . . . . . . . . . . . . . . 29

3.4 Coding inside Juum . . . . . . . . . . . . . . . . . . . . . . 31

3.5 Use of Juum in artistic contexts . . . . . . . . . . . . . . . . . . 31

3.6 Conclusions . . . . . . . . . . . . . . . . . . . . 33

Chapter 4: Artistic Methodology . . . . . . . . . . . . . . . 34

4.1 Artistic Questions and Concerns . . . . . . . . . . . . . . . . 34

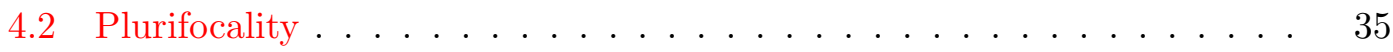

4.3 Public Art and Open Space . . . . . . . . . . . . . . . . 36

4.4 Journeys and Errands . . . . . . . . . . . . . . . . . . . . 36

4.5 Improvisation, Interactivity, and the Open Work . . . . . . . . . . 37

4.6 Sound Art . . . . . . . . . . . . . . . . . . . . . 40

4.7 Electroacoustic Music . . . . . . . . . . . . . . . . . 42

4.8 The Electronic Sound of the Analog World, Paradigms, and Formats . . 42

4.9 Mechanical Music . . . . . . . . . . . . . . . . . . . . . 43

4.10 Data Sonification, Translation, Mapping, and Modulation . . . . . . . . 44

4.11 Science and Technology as Manifestations of Art . . . . . . . . . . 46

4.12 Obsolescence . . . . . . . . . . . . . . . . . . 46

4.13 The Ocean . . . . . . . . . . . . . . . . . 47

4.14 Chapter conclusion . . . . . . . . . . . . . . . . . . 48

Chapter 5: Preliminary Work . . . . . . . . . . . . . . . . . . . 49

5.1 Explorations and Sketches . . . . . . . . . . . . . . . . . 49

$5.1 .1 \quad$ Jason Expedition . . . . . . . . . . . . . . . . . . . 50

5.1 .2 Sea Journey at the Wood Ship . . . . . . . . . . . . . . 51

5.1 .3 Exhalation-Water . . . . . . . . . . . . . . . . . . 51

5.1 .4 First Expedition, Imagery . . . . . . . . . . . . . . . . 55

5.2 Initial Proposals . . . . . . . . . . . . . . . . . . 58

5.2.1 Proposal One: Laboratory Performance over the Sea . . . . . . 58

5.2 .2 Proposal Two: Multichannel Telematic . . . . . . . . . . . . 60

5.3 Axial's Concerns . . . . . . . . . . . . . . . . . . . 62

5.4 Undersea Soundscape . . . . . . . . . . . . . . . . . . . . . . . . . . . . 63

5.4 Recording Preparation . . . . . . . . . . . . . . . 63

5.4 .2 The Recording Gesture . . . . . . . . . . . . . . . 68

5.4 .3 The Recorded Material . . . . . . . . . . . . . . . . . 70

5.5 Sound Recordings on the Thompson . . . . . . . . . . . . . 71 
5.6 Data at the Ship . . . . . . . . . . . . . . . . 72

5.6 .1 DAS ........................... 73

5.6 .2 Bathymetry ...................... 76

5.6 .3 Sub-bottom Profiles . . . . . . . . . . . . . . 77

5.7 Cloud Synthesis . . . . . . . . . . . . . . . . . . . . . 78

5.8 Conclusions . . . . . . . . . . . . . . . . . . . . . 79

Chapter 6: Composition and Deployment . . . . . . . . . . . . 80

6.1 Ideas About Composition and Creativity . . . . . . . . . . . . . 81

6.2 Compositional Process . . . . . . . . . . . . . . . . . . . 82

6.2.1 Planning of the Physical Manifestation . . . . . . . . . . . . . 84

6.2.2 Compositional Process of the Sound Material . . . . . . . . . 84

6.3 Axial Structure and Parts . . . . . . . . . . . . . . . . . 95

6.3.1 Physical Manifestation . . . . . . . . . . . . . . 95

6.3 .2 Compositional Structure . . . . . . . . . . . . . . 97

6.4 Deployment . . . . . . . . . . . . . . . . . . . . . . 98

6.4.1 Location . . . . . . . . . . . . . . . . . . . . . . . . . . . . . . . . . . . . . . .

6.4 .2 Setup .............................. 100

6.4.3 Presentation ........................ 101

6.4.4 Feedback and Comments . . . . . . . . . . . . 105

6.5 Chapter Conclusions . . . . . . . . . . . . . . . . . . 106

Chapter 7: Conclusions and Future Work . . . . . . . . . . . . 108

7.1 Conclusions . . . . . . . . . . . . . . . . . . . . . . . 108

7.2 Contributions . . . . . . . . . . . . . . . . . . 109

7.3 Future Work . . . . . . . . . . . . . . . . . . . . . 110

7.3 .1 Delta $16.57 /-99.00 \ldots \ldots \ldots \ldots 110 \ldots \ldots$

7.3 .2 ATL . . . . . . . . . . . . . . . . . 114

Appendix A: Digital Materials . . . . . . . . . . . . . . . . . 116

Appendix B: Plurifocal Events Controller Specification . . . . . . . . . . . 117

B.1 Technology of the Plurifocal Events Controller . . . . . . . . . . . . . 117

B.1.1 Microcontrollers . . . . . . . . . . . . . . . . 117

B.1.2 ZigBee and BitCloud . . . . . . . . . . . . . . . . 118

B.2 Fabrication of Electronics . . . . . . . . . . . . . . 120

B.2.1 Prototype . . . . . . . . . . . . . . . . 120

B.2.2 First Version . . . . . . . . . . . . . . . 120 
B.2.3 Second Version . . . . . . . . . . . . . . . . . . . . . . 122

B.3 Fabrication of Mechanical Components . . . . . . . . . . . . . . 125

B.4 Applications of the Plurifocal Events Controller . . . . . . . . . . . . . . 131

B.5 Materials . . . . . . . . . . . . . . . . . 133

B.6 Diagrams . . . . . . . . . . . . . . . . . . . 135

B.7 Protocol . . . . . . . . . . . . . . . . . . . . . . . . . 141

B.7.1 ZigBee Protocol . . . . . . . . . . . . . . . . . . . 141

B.7.2 Rhythm Protocol . . . . . . . . . . . . . . . . . . . 141

B.8 Code . . . . . . . . . . . . . . . . . . . . . . . . . . 141

Appendix C: Juum scores . . . . . . . . . . . . . . . . . . . . . . 150

Appendix D: Cloud Synthesis . . . . . . . . . . . . . . . . . . . 158

D.1 Amazon Web Services . . . . . . . . . . . . . . . . . . . 158

D.2 Grails . . . . . . . . . . . . . . . . . . . 158

D.3 The Cloud Synthesis Infrastructure . . . . . . . . . . . . . . 158

D.4 Audio processes . . . . . . . . . . . . . . . . . . . . . 162

Appendix E: Marine Geology Context and the Ocean Observatory Initiative . 173

E.1 Art, Ocean, and Myths _. . . . . . . . . . . . . . . . . 173

E.2 Marine Geology . . . . . . . . . . . . . . . . . . . . . . . 175

E.2.1 Oceanography . . . . . . . . . . . . . . . . 175

E.2.2 Earth Origins . . . . . . . . . . . . . . . . . . 176

E.2.3 Earth's Structure and Composition . . . . . . . . . . . . 178

E.2.4 Movement of the Continents and Plate Tectonics . . . . . . . . . 178

E.2.5 Hydrothermal Vents . . . . . . . . . . . . . . . . . . . . 180

E.2.6 Bathymetry of the Seafloor . . . . . . . . . . . . . . . . 182

E.2.7 Sea Water . . . . . . . . . . . . . . . . . . . . . . . . . . . . . 182

E.2.8 Sound in Seawater . . . . . . . . . . . . . . . . . . . . 185

E.3 The Oceans Observatory Initiative . . . . . . . . . . . . . . . 186

E.3.1 Ocean Observatories Initiative's Regional Scale Nodes . . . . . . 189

E.3.2 History . . . . . . . . . . . . . . . . . . . . . . . . 190

E.3.3 Research Within the OOI . . . . . . . . . . . . . . . . . . . 191

E.3.4 Technology at the OOI . . . . . . . . . . . . . . . . 191

E.3.5 Expeditions of the OOI . . . . . . . . . . . . . . 193

Bibliography . . . . . . . . . . . . . . . . . . . . . 199 


\section{LIST OF FIGURES}

Figure Number $\quad$ Page

1.1 Conceptual elements . . . . . . . . . . . . . . . . . 2

1.2 Axial container . . . . . . . . . . . . . . . . 3

2.1 Equipment distribution of OnSpace by Juan Pampin . . . . . . . . . . . . . . 8

2.2 Fragment of Calandra by Buñuel . . . . . . . . . . . . . . . . . . 10

2.3 Initial section of Pulsante Fragor by Llorenç Barber . . . . . . . . . . . 12

2.4 Sound paths for Poème électronique . . . . . . . . . . . . . . . 15

2.5 Sketch for Swarm distribution . . . . . . . . . . . . . . . . 19

2.6 Tell-Tale Piano at the McLeod residency . . . . . . . . . . . . . . 20

3.1 Field canvas . . . . . . . . . . . . . . . . . . . 25

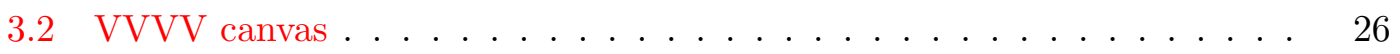

3.3 Juum initial sketch . . . . . . . . . . . . . . . . . . . . 27

3.4 Logic of Juum software . . . . . . . . . . . . . . . . . . . . . . . . . . . . . . . . . . .

3.5 Juum elements . . . . . . . . . . . . . . . . . . . . . . . 30

3.6 Juum software during concert . . . . . . . . . . . . . . . . . 33

4.1 Fork2 by Richard Serra . . . . . . . . . . . . . . . 37

4.2 Recollector by James Coupe . . . . . . . . . . . . . . . . . . . . 39

4.3 Entanglement by Juan Pampin, Eunsu Kang, and Joel S. Kollin . . . . 40

4.4 Score of Exchange by Richard Karpen . . . . . . . . . . . . . . . . . . . . . . . . 43

4.5 Trimpin's player piano . . . . . . . . . . . . . . . . . . . 44

4.6 Testpattern by Ryoji Ikeda . . . . . . . . . . . . . . . . . . . 45

4.7 Old ships . . . . . . . . . . . . . . . . . . 47

5.1 Jason expedition in $1993 \ldots \ldots \ldots$. . . . . . . . . . . . 50

5.2 First sketches for the sea journey . . . . . . . . . . . . . 52

5.3 Scheme of sea journey . . . . . . . . . . . . . . . . 52

5.4 The Silver Wave . . . . . . . . . . . . . . . . 53

5.5 Puget sound nautical chart . . . . . . . . . . . . . 53

5.6 Exhalation-Water's audio analysis box . . . . . . . . . . . 55

5.7 Picture of Lumens expedition $(\mathrm{A}) \ldots \ldots \ldots$. . . . . . . . . . 56

5.8 Picture of Lumens expedition $(\mathrm{B}) \ldots \ldots \ldots \ldots$ 
5.9 Picture of Lumens expedition $(\mathrm{C}) \ldots \ldots \ldots \ldots \ldots$

5.10 Picture of Lumens expedition (D) . . . . . . . . . . . . 57

5.11 Sketch of proposal one . . . . . . . . . . . . . . 59

5.12 Diagrams of proposal two . . . . . . . . . . . . . . . 60

5.13 Sketch of proposal two . . . . . . . . . . . . . . . . . . . . . . . . . . . . 61

5.14 Container at ship . . . . . . . . . . . . . . . 62

5.15 Preparation of recording . . . . . . . . . . . . . . . 65

5.16 VentPhone and $\mathrm{H} 2$ recorders . . . . . . . . . . . . . 66

5.17 Recorder in tripod . . . . . . . . . . . . . . . . . . . . . . . . . . . . 67

5.18 Jason arm with hydrophone . . . . . . . . . . . . . . . 68

5.19 Guidance of Jason . . . . . . . . . . . . . . . . . . . . . . . . . . . . . . . . . . . .

5.20 Jason's cameras . . . . . . . . . . . . . . . . . . . . 70

5.21 Waveform of underwater recordings . . . . . . . . . . . . . . . . . . . . . . . . 71

5.22 Sound recording at Thompson . . . . . . . . . . . . . . 73

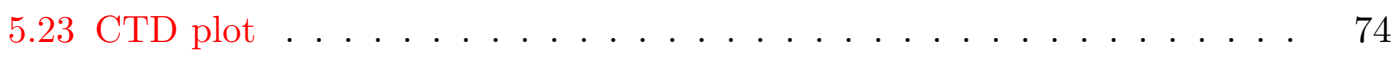

5.24 Plot of sound velocity . . . . . . . . . . . . . 76

5.25 Bathymetry of Axial seamount . . . . . . . . . . . . . . . . . . 77

5.26 Sub-bottom profile . . . . . . . . . . . . . . 78

6.1 Axial's production timeline . . . . . . . . . . . . . . 83

6.2 Sketches of ideas . . . . . . . . . . . . . . . . . 85

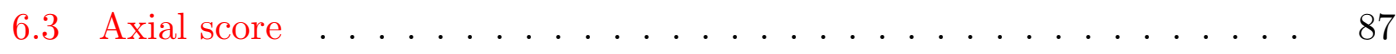

6.4 Axial's diagram connections and parts . . . . . . . . . . . . 96

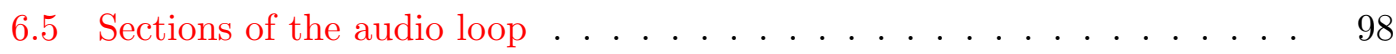

6.6 Container's view located at Center for Urban Horticulture . . . . . . . . 99

6.7 Map of the Center for Urban Horticulture . . . . . . . . . . . . . . . . . 101

6.8 Solenoid mounted on the wall of the container . . . . . . . . . . . . . 102

6.9 Set of four solenoids and one PEC . . . . . . . . . . . . . . . . 102

6.10 Wall of solenoids, PEC, and power supply . . . . . . . . . . . . . . 103

6.11 Internal Setup of Axial . . . . . . . . . . . . . . . . . . 103

6.12 Inside view of Axial installation $(\mathrm{A}) \ldots \ldots \ldots$. . . . . . . . 105

6.13 Inside view of Axial installation $(\mathrm{B}) \ldots \ldots \ldots \ldots$

6.14 Postcard invitation . . . . . . . . . . . . . . . . . . 107

7.1 Delta location . . . . . . . . . . . . . . . . . . . . 111

7.2 Sea and fresh water at the Copala delta . . . . . . . . . . . . 112

7.3 Copala river . . . . . . . . . . . . . . . . . . . . . . . 113

7.4 Location for the Delta main node . . . . . . . . . . . . . . . . . 113 
7.5 Distribution of sonic elements in ATL . . . . . . . . . . . . . . 115

B.1 Mesh topology of a BitCloud Network . . . . . . . . . . . . . . . 119

B.2 First prototype of the PEC . . . . . . . . . . . . . . . . 121

B.3 First version of the PEC . . . . . . . . . . . . . . . . . . . 122

B.4 Second version of the PEC . . . . . . . . . . . . . . . . 123

B.5 Second version of PEC finished . . . . . . . . . . . . . . . . . 124

B.6 Initial sketches for solenoid mechanism . . . . . . . . . . . . . . . . 126

B.7 Sketch of solenoid mechanism . . . . . . . . . . . . . . . . 127

B.8 Wood bases . . . . . . . . . . . . . . . . . . . . 127

B.9 Solenoids . . . . . . . . . . . . . . . . . . . . . . 128

B.10 Drilling stricks . . . . . . . . . . . . . . . . . . 128

B.11 Damper heads . . . . . . . . . . . . . . . . . . . . . . . 129

B.12 Springs for solenoids . . . . . . . . . . . . . . . . . . . . 129

B.13 Assembly of bases . . . . . . . . . . . . . . . . . . 130

B.14 Set of solenoids and sticks . . . . . . . . . . . . . . . 130

B.15 Metaphors for Dead Pianos . . . . . . . . . . . . . . . . . 132

B.16 Objetos resonantes . . . . . . . . . . . . . . . . . . 133

E.1 Theatrum Orbis Terrarum . . . . . . . . . . . . . . . . . . . . . 174

E.2 Alexander Calder, Sea Life _. . . . . . . . . . . . . . . . . 176

E.3 First physiographic chart of the world's oceans . . . . . . . . . . . . 179

E.4 Earth's lithosphere plates . . . . . . . . . . . . . . . . . . . . 180

E.5 El guapo hydrothermal vent . . . . . . . . . . . . . . . . . . . 181

E.6 Bathymetry of the world's ocean basins . . . . . . . . . . . . . 183

E.7 OOI RSN Essential Elements . . . . . . . . . . . . . . . . . 187

E.8 OOI Network plan . . . . . . . . . . . . . . . . . . . . . . . . 189

E.9 Enlighten Leg 2 Science Party . . . . . . . . . . . . . . . . . . . . . . . . . . . . 195

E.10 Axial Caldera infrastructure . . . . . . . . . . . . . . . . . 196 


\section{LIST OF TABLES}

Table Number $\quad$ Page

2.1 Pieces with spatial preocupations . . . . . . . . . . . . . . 14

5.1 Recording System Specifications _. . . . . . . . . . . . . . . 64

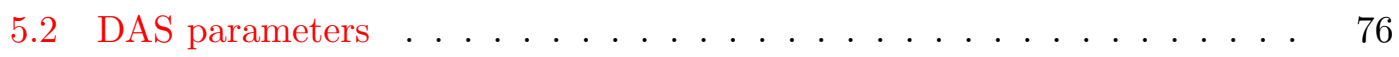

A.1 List of materials . . . . . . . . . . . . . . . . 116

B.1 PEC list of components . . . . . . . . . . . . . . 135

B.2 PEC list of power module . . . . . . . . . . . . . 136

B.3 PEC header protocol . . . . . . . . . . . . . . . . . . 147

B.4 PEC body protocol . . . . . . . . . . . . . . . . . . . 148

B.5 PEC examples of protocol use . . . . . . . . . . . . . . . 149

E.1 Major constituents of seawater . . . . . . . . . . . . . 183

E.2 Abundance of gases . . . . . . . . . . . . . . . . . . . . . . . 184 


\section{ACKNOWLEDGMENTS}

Profound and sincere thanks to Juan Pampin for the support and trust in the work of your students, and for the opportunity you gave me to achieve a Ph.D. at the Center for Digital Art and Experimental Media (DXARTS). Thanks for sharing your experience and knowledge about sound. Thanks to Richard Karpen for the vision of creating such an amazing program and for the constant observations on the essence of art. Thanks to James Coupe and all the faculty at DXARTS for the courses and the support.

I would like to share my profound gratitude to Bruce Hemingway professor at the Computer Science department for his support and help. His course and coaching provided important guidance toward the realization of the $\mathrm{Ph}$.D. I also would like to thanks John Delaney, professor of Oceanography at the University of Washington for his extraordinary support of this project. This work would not have happened without his openness to exploration and experimentation.

Thanks to Tim Crone, Erick Thompson, Brian Mayton, Nicolas Varchausky, Stelios Manousakis, Tivon Rice, James Hughes, Michael McCrea, Josh Parmenter, Heather Raikes, Eunsu Kang, Donal Craig, Annabel Castro, Los Padrinos Carlos y Rubi, Susie \& Pancho, Mirta \& Eloy, Los Capurro, Steve Peters, Blake \& Rosio, Lele Barnett, Joan Rabinowitz \& Levi Fuller, Lauren Fortune, Deborah Kelley, Mitch Elend, Nancy Penrose, Trimpin, Ginger Armbrust, Robley Carr, and all the people, friends, and Seattlelites who made this project possible and who made living in Seattle an extraordinary experience. Thanks to Arcangel Constantini, Grace Quintanilla, Juan Diaz Infante, and Alejandro Ramos who, in one way or another, have contributed to the creation of artwork in Mexico City. Thanks to Gabriela and Mateo, the lights of my life, not only for daily companionship and support but for giving reason to my existence. Gracias a mi musa que está en todos lados y a todas horas desde hace varias vidas. 


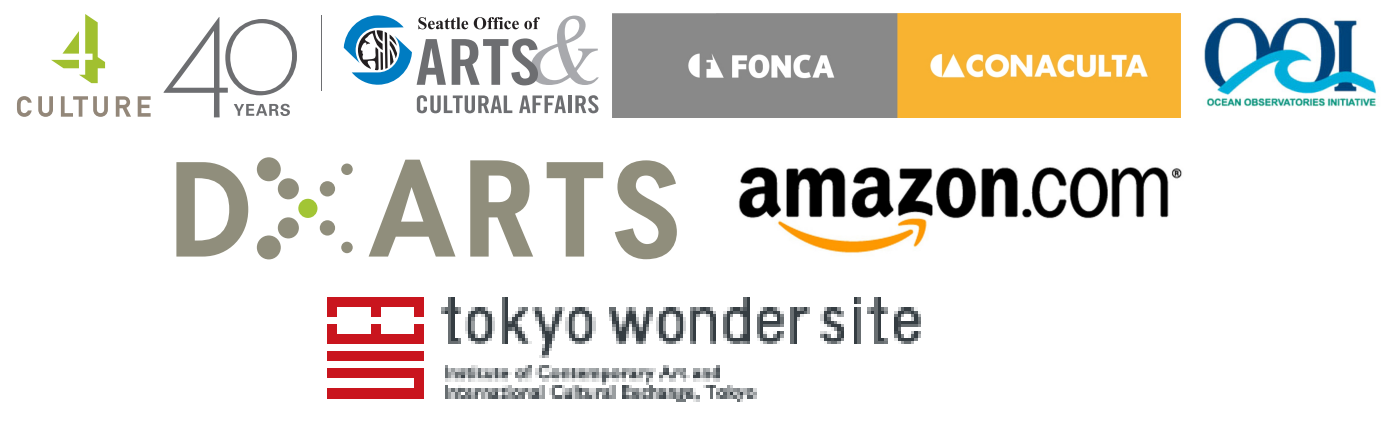

Elements of this project were supported by the following organizations:

- The City of Seattle Office of Arts \& Cultural Affairs

- 4 Culture

- The Ocean Observatories Initiative

- The Center for Digital Art and Experimental Media

- Proyecto apoyado por el Fondo Nacional para la Cultura y las Artes bajo el programa de Jóvenes Creadores 2008

- Proyecto apoyado por el Fondo Nacional para la Cultura y las Artes bajo el programa de Residencias Artísticas 2010 con el cual se realizó una residencia en Tokyo Wonder Site

- Proyecto realizado a través del Programa de Apoyo a la Producción e Investigación en Arte y Medios 2010 del Centro Multimedia del Centro Nacional de las Artes

- Use of Amazon Cloud was made possible by the Amazon Education Grant.

The author is a current member of the Sistema Nacional de Creadores de Arte of Mexico. 


\section{DEDICATION}

To my first teachers, Jiu and Chichi 
Chapter 1

\section{INTRODUCTION}

And God said, "Let there be an expanse between the waters to separate

water from water".

Genesis 1:6

This introductory chapter explains the logic, development, and compositional process of a sound art work created as a final piece for the doctoral program in Digital Arts and Experimental Media (DXARTS). The program is offered by the Center for Digital Arts and Experimental Media at the University of Washington.

By its nature, the entire process of creating the work was full of exploration, challenges, and open questions. The experimentation, the original problem solving, and the consideration of aesthetic implications and relationships between different fields were core parts of the creation of the work. This work, named Axial, continues the artist's personal research into the central role sound plays in creating exploratory dialogs between art, science, technology, physical space, and the ocean.

\subsection{What Is Axial}

Axial is an installation that uses sound to talk abstractly about the power of the ocean and the deep sea. Its name comes from the Axial Volcanic Ridge located on the Juan de Fuca Ridge where the sound recordings and oceanographic data were obtained with the support and help of the Ocean Observatories Initiative. Among the recordings, we encounter a set of sound registers obtained from hydrothermal vents located at the deep sea floor.

The material obtained during the oceanographic expedition was later interpreted and converted into a sound installation, in which a cargo container was employed as a 


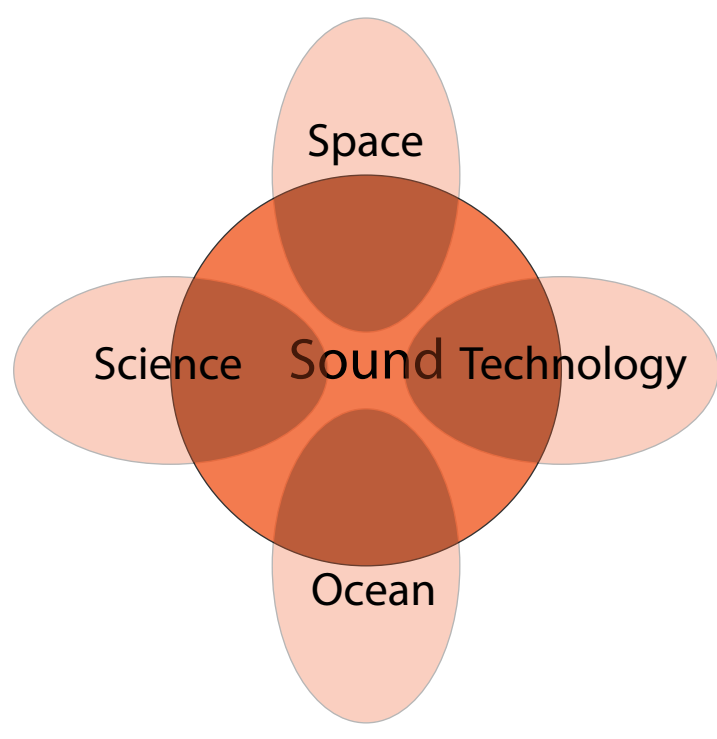

Figure 1.1: Conceptual elements.

sonic resonator as well as a physical delimiter that conceptually simulates the void and border the sea generates with the rest of the environment.

Axial is also a sonic exploration where two distinct sonic layers merge and dialog in order to generate the acoustic experience. On one side, a set of 80 evenly distributed solenoids with sticks attached to them hit the walls of the shipping container, producing rhythmic patterns. At the same time, a second layer of digital material is generated by six speakers hanging from the walls of the container. The container was placed in an open field in order to maximize the sensation of void, distance, and open space. The selected location was the Center for Urban Horticulture at the University of Washington.

The creation of this work required an artistic exploration that lasted more than two years. Axial is indebted to a wide set of influences including the work of Juan Pampin, Richard Karpen, and James Coupe. It follows the paradigm of Trimpin's work and it is based on the sonic ideas of Llorenç Barber about sound in the public space. In addition to the aesthetic exploration, a piece of software and a set of electronic devices were crafted and gradually developed in order to produce this work. 


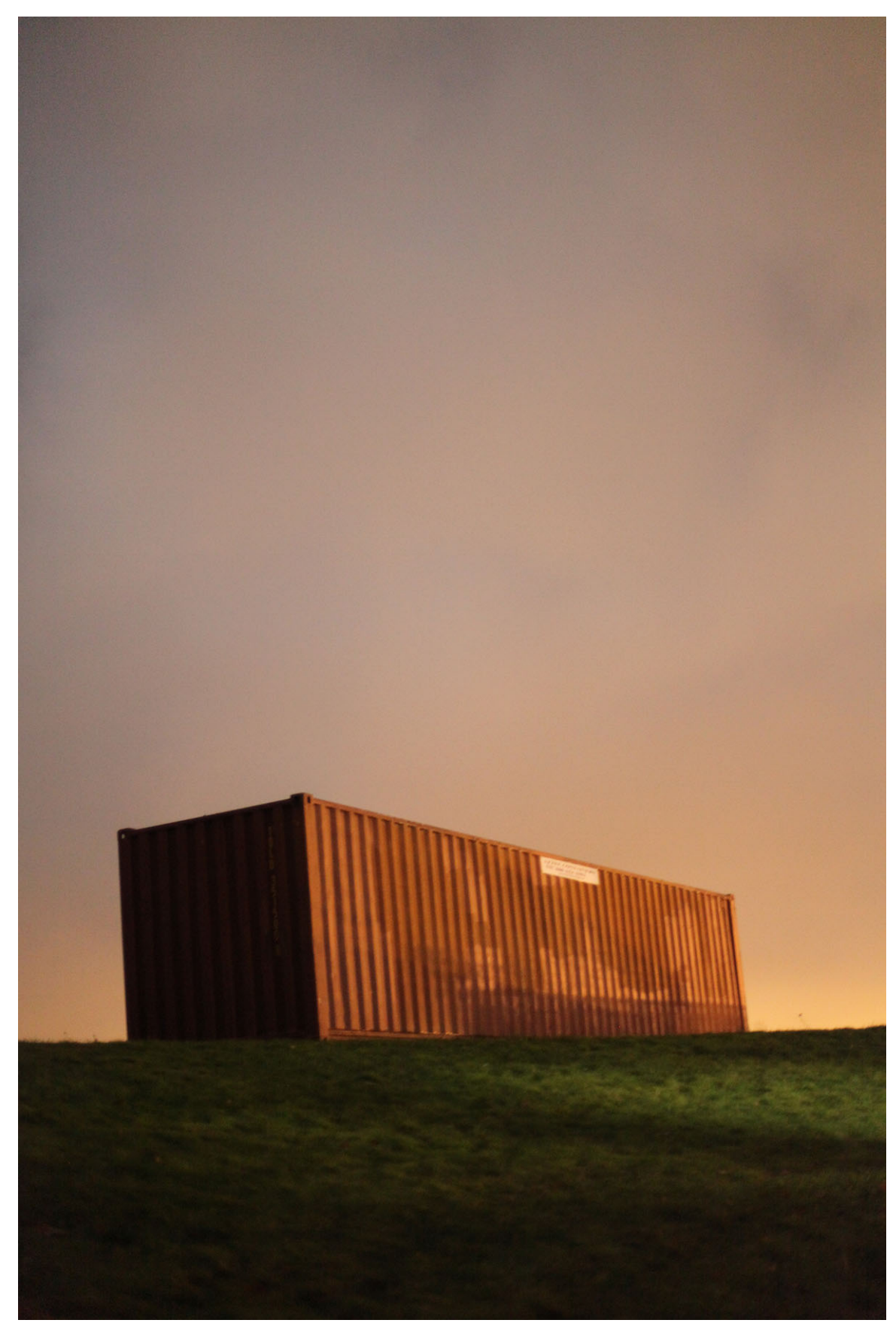

Figure 1.2: Axial container. 


\subsection{Organization of the Thesis}

This document is divided into seven chapters and five appendixes. This chapter condenses the logic of the entire document and offers a succinct description of the project. This chapter functions as an abstract and nutshell description of the artwork and the creative process.

The chapter Digital Plurifocality and the Plurifocal Events Controller presents a description of the core elements of sound and space. The element of space was of extreme importance during the creative process, not only as a physical distribution of sound sources, but most importantly as a conceptual logic and interconnected variable.

In the same chapter, the Plurifocal Events Controller is described and detailed. This technology was created for this work with the goal of having an infrastructure that permits the generation and manipulation of analog sound sources spread over large areas. The concept of digital plurifocality was important for the creation of Axial and a constant dialog between such logic and the artwork was part of the evolution of ideas that gave shape to the artwork. The concept is therefore also presented in this chapter, explaining the influences and previous experience that led to this particular work. The technical details and the building process of the electronics of such technology are presented in Plurifocal Events Controller Specification.

In order to control, shape, and synchronize both the digital audio layer and the mechanical solenoids, a piece of software was created. The chapter Juum Software explains the direct influences, logic, and capabilities of this piece of software, which follows the paradigm of timeline representation and offers a personal solution of merging time control with scripting programming. Juum derives most of its influence from the Field software created by Marc Downie. Juum scores presents a set of digital scores created with Juum and presented by the artist in shows and concerts while a student at DXARTS.

Axial is a complex, multilayered piece and is directly influenced by many previous works and proposals of numerous artists and creators. Axial gives a personal interpretation to many concerns and artistic preoccupations including, among others, the meaning of the public space, the aesthetics of data sonification, the potential and limitations of 
mechanical music, the meaning of plurifocal sound distribution, and the intersections of art, science, and technology. The chapter Artistic Methodology describes such concerns pointing out artistic references and direct influences to the work.

Axial was created over a long period of time. Several years passed between the seminal idea and the deployment of the final version. Over this period of time, several manifestations were tried out and several proposals submitted for discussion and analysis to the faculty committee. Axial did not bloom from the void, and the authors's concern to work with the ocean and the deep sea was old. Between the initial oceanographic expedition during high school in 1993, to the Enlighten expedition in 2010, several projects related to water, journeys, and sound were produced including Exhalationwater, a piece for the general exam of the DXARTS program. The chapter Preliminary Work presents a detailed description of the process that led to the final version of Axial.

The same chapter also describes the process of recording the hydrothermal vents with the help and support of the oceanographic party during the oceanographic expedition. The chapter explains how such recordings, in addition to other scientific data, were employed for the creation of the Axial sound layer. Among the information we encounter DAS data, bathymetry information, and sub-bottom profiles which together were used as modulators of sonic parameters. A marine context about these and other oceanographic elements is presented at Marine Geology Context and the Ocean Observatory Initiative.

The chapter also describes how the data was parsed using an scripting language called Groovy that controlled SuperCollider sound renderers, all running on a cloud computer system leading to the concept of Cloud Synthesis. The technical details of the entire rendering system are presented in Cloud Synthesis.

Chapter Composition and Deployment presents the compositional process of the final work. Starting from some ideas about composition and creativity, the chapter relates how each element of the work was produced and how all the layers were interconnected sonically, artistically, and conceptually. The organization, structure, and parts of the work are also described, including how the final tuning of the piece was created on the exhibition site. The chapter also describes the deployment process including the location selection, setup, and public presentation.

Chapter Conclusions and Future Work offers a set of final thoughts explaining the 
experience of having created, built, and shown the work. The chapter analyzes the experience, pointing out the elements that the author considers the right decisions as well as elements that could have been different or could have worked better with changes, variations, simplifications, or extensions. The chapter finishes with the description of two works in progress that have been directly influenced by Axial. 
Chapter 2

\title{
DIGITAL PLURIFOCALITY AND THE PLURIFOCAL EVENTS CONTROLLER
}

\author{
"Aprendí a base de equivocaciones, \\ de estudiar las formas de propagación y reflexión de la onda sonora \\ cuando tropieza con una pared, con la humedad, con el viento. \\ ...Ya no había que distinguir entre virtuosos y no virtuosos, \\ sino entre atentos y no atentos." \\ Llorenç Barber
}

This chapter presents inspirations, references, and thoughts about digital plurifocal music, a concept that embraces a set of sonic logics derived from plurifocal music, and which was the basis for many of the aesthetic decisions of Axial. The chapter also discusses historical references regarding sound and space.

The chapter also presents details of a custom electronic device called the Plurifocal Events Controller, which was designed and fabricated by the author with the intention of creating a kit that allows the wireless control of analog mechanical devices such as motors and solenoids.

\subsection{Inspirations}

One of the strongest conceptual bases for Axial is the concept of digital plurifocality, a hybrid term used to describe not only a type of composition and creation but also a type of listening. Where does this term come from? Why was it important for the author to describe it and translate it into the digital world? In this section, the inspirations that led to the term digital plurifocality are explained by way of describing its origins and also as a way of describing its internal logic. 


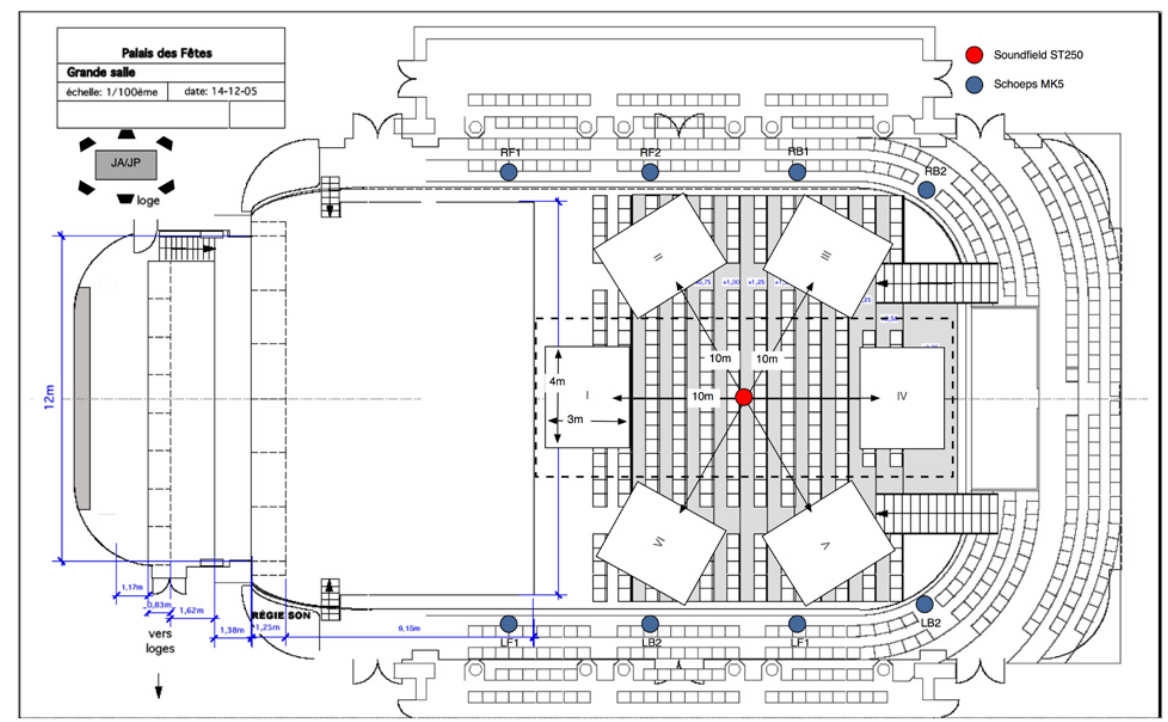

Figure 2.1: Equipment distribution of OnSpace by Juan Pampin.

\subsubsection{The Work of Pampin, Karpen, and Coupe}

When the author entered the DXARTS program, he had the opportunity to study and observe the creative process of Juan Pampin, Richard Karpen, and James Coupe. In the course of creating Axial, discussion and feedback with these three artists but most importantly the example of their artistic work was crucial in shaping, analyzing, and developing a profound concern for the relationship between sound and space, and more generally about art in the digital era.

Listening to the multichannel works of Karpen and Pampin during DXARTS concerts was vital to the appreciation of proposals where space works as an interconnected element in constant dialog with the sonic material. Among all the works that DXARTS students were exposed to, the piece OnSpace by Juan Pampin was the work that influenced the author most because it handled the use of space from many perspectives.

Other powerful influences on the work of the author were the courses "Sound and Space" and "Sound Art," offered by Juan Pampin as part of the academic program. During these courses, advanced students in the program discussed topics and concerns about sound and space and also about sound art in general. During reunions the group 
focused on concerns regarding the use of sound, the meaning of sound in modern society, and the borders between artistic fields of different media.

Another influence on this proposal is the work of James Coupe and his course "Mechatronic Art." In this course, students produced a set of works that used new media in order to express a profoundly artistic narrative. Criticism and challenge were employed as incentives to search for original works. This course began an exploration of the core concepts not only of digital plurifocality but also of the entire Axial project. Every step and idea of the project was questioned, gradually forcing the author to understand that strong projects required a profound exploration. In the words of Professor Pampin, the important element was to find the appropriate questions.

\subsubsection{La Ruta del Tambor y el Bombo}

In 2006, while student of Music Technology at the Pompeu Fabra University, the author had a Spanish roommate who invited me to his hometown of Andorra de Teruel during the Catholic celebration of Semana Santa. During this celebration, the entire population of the town takes their snare and bass drums and plays rhythmic patterns through the night. The children of Andorra de Teruel learn to play the snare drum at school in order to preserve this celebration, which lasts for two days. The celebration starts with silence until the Municipal President plays a huge bass drum on the balcony of the government palace. This moment is called "romper la hora" (breaking the hour). The experience of having a stony silence in a crowd of hundreds of drums and suddenly hearing all the drums begin to play together is spectacular. In this sonic cult to God, throughout the night small groups of people stroll around the narrow streets of the town. Each group is playing a set of rhythmic patterns that distinguish them from the rest of the groups. After several hours, the entire town walks up a hill to the church and the groups dissolve, forming two parallel lines. Between this sonic corridor the figure of Jesus will be moved from downtown to the church.

Later, after listening this spectacular experience, I learned that this celebration started in the 12 th century and spread over nine towns of Spain. They are now known as the "ruta del tambor y el bombo" (the route of the snare and bass drum) and are considered a national festivity and touristic attraction. The aesthetic and sonic attraction 


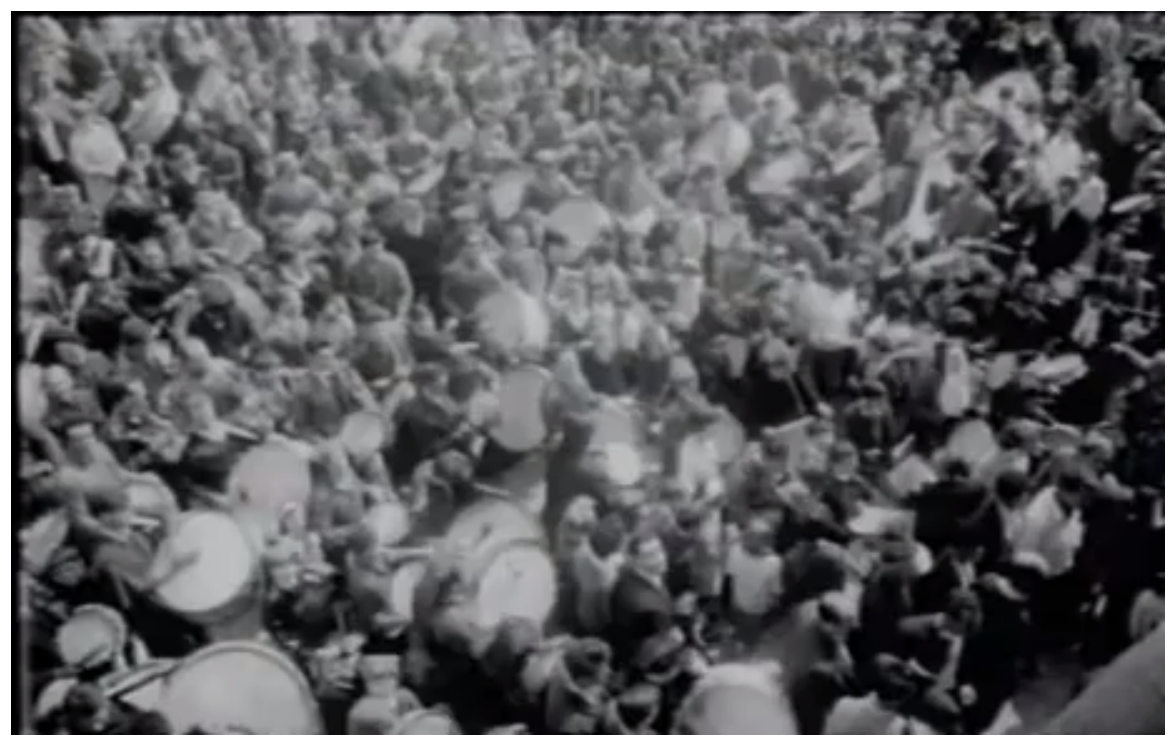

Figure 2.2: Fragment of Calandra by Luis Buñuel, 1966.

of this celebration inspired several Luis Buñuel's movies. La Edad de Oro, Nazarin, and Simón del Desierto use sound recordings of the celebration and Calandra has long shots of the celebration.

Having a special interest in the spatialization of sound, attending this stunning experience was extremely important to the author in shaping a sound imagery. How to reinterpret it? How to appropriate and translate the experience to a personal gesture? These questions were also prominent in my created work since then.

\subsubsection{Ecos del Milenio}

In 1999, while the author was a student of music at the Escuela Nacional de Música, the piece Ecos del Milenio by the Spanish composer Llorenç Barber was performed at the Zocalo of Mexico City. Several students of music were invited to help perform the piece. The work required about 100 performers because one person was required for each bell of the cathedral. The experience of working hand in hand with the composer during the rehearsal and the concert opened an entirely new way of thinking about sound, of thinking about sound in relationship to space, and more important, of thinking about 
spatial sound in relationship to collective rituals.

The small town of Cholula, near Mexico City, contains more than 300 churches. This gave and opportunity to Barber to work with an enormous amount of sound sources, and in 1993 he composed "Vaniloquio Campanero" for World Music Days. The work became something of a tradition and tourist attraction and was performed every year for a long time. On some of his visits to Mexico, Barber gave talks about his work and his concepts at the School of Music. His idea of distributing sound over big areas has been a strong influence on the author's work.

\subsubsection{Previous Work by the Author}

Between 1994 and 2001, the author was a student of music and had the opportunity to study with the Mexican composer Julio Estrada. He encouraged his students to explore sound with outside logics far away from the mainstream. As a young student, listening to his work "Canto Naciente" (1975-1978) performed live was extremely inspiring. This piece distributed eight brass players around the hall. The piece plays with the panning of sounds between the instruments. Using the physical movement of sound became a strong interest of the author and this element has become more important over time. The interest of the author in the use of the spatialization of sound resulted during that time in the article El espacio físico como variable estructural en música[36] and the thesis "GAB: Sistema de reinterpretación para pianos" [37]. While a student of Estrada, two other inspirations from that time were important: the visit of Trimpin and the symposium about Conlon Nancarrow's work. Trimpin was the first person I had ever met in person that build mechanical devices that produced sound using custom electronics; Nancarrow's work was my first approach to a proposal that required building technical solutions in order to solve sonic ideas.

\subsection{Digital Plurifocality}

The term digital plurifocality has been coined as a way to express an interest in using digital technologies to extend the possibilities and sonic aesthetics of plurifocal music. The technological aspect of this thesis intends to contribute to the field by offering a personal answer to the question: Can digital technologies support and help to enrich 


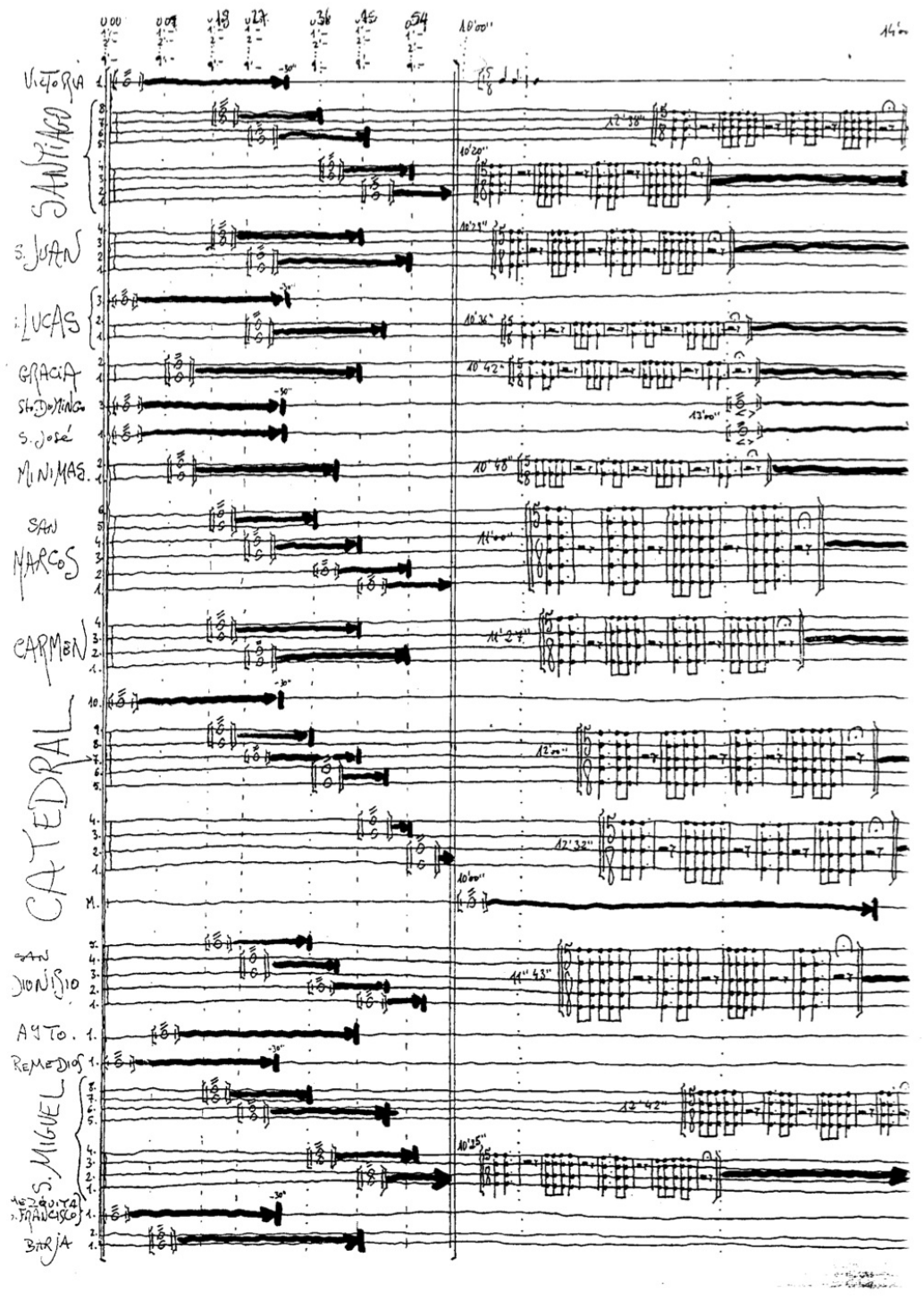

Ejemplo 6. Introducción de Pulsante fragor (Jerez, septiembre de 1996) $0^{\prime}$-14".

Figure 2.3: Initial section of "Pulsante Fragor" by Llorenç Barber, 1996. 
the notions and sonic possibilities of plurifocal music?

\subsubsection{Physical Space as a Musical Variable}

It is extremely important to mention that the aesthetic sonic concepts of spatial sound rely on a strong and long tradition regarding the use of the physical space in sound composition. The perceptual recognition of location in sound is physiologically well known and documented[19][18]. In music, the use of distributed sound sources has been present for hundred of years. We can point to the works of Giovanni Gabrieli and the polichoral style of the Venetian School as a key example previous to the 14th century, to mention just one. Table 2.1 lists some works with a notable preoccupation regarding the use of physical space ${ }^{1}[14]$.

As will be seen in the following chapters, Axial is a work with a preoccupation of sound in several levels of interpretation, from the use of spatialize sound to the reinterpretation of the ocean area, from the use of a close container in the land to the recording of distant regions.

\begin{tabular}{l|l}
\hline Composer & Piece \\
\hline Gustav Mahler & "The Last Trump" in Symphony No.2 \\
Charles Ives & Symphony No. 4 \\
Béla Bartók & Music for Strings, Percussion, and Celeste (1937) \\
Henry Brant & Antiphony I (1953/1968) \\
Henry Brant & Millennium II (1954) \\
Iannis Xenakis & Pithoprakta (1955-56) \\
Edgar Varèse & Poème électronique (1958) \\
Luciano Berio & Circles (1960) \\
Elliott Carter & Doule Concerto (1961) \\
Karlheinz Stockhausen & Carré (1959/1960) \\
Karlheinz Stockhausen & Musik in space (1959/1961)
\end{tabular}

\footnotetext{
${ }^{1}$ It is beyond the scope of this document to delve into the use of space in music but the thesis "Space and Spatialization in Contemporary Music; History and Analysis, Ideas and Implementations" is a valuable reference to explore the notion of sound in music.
} 


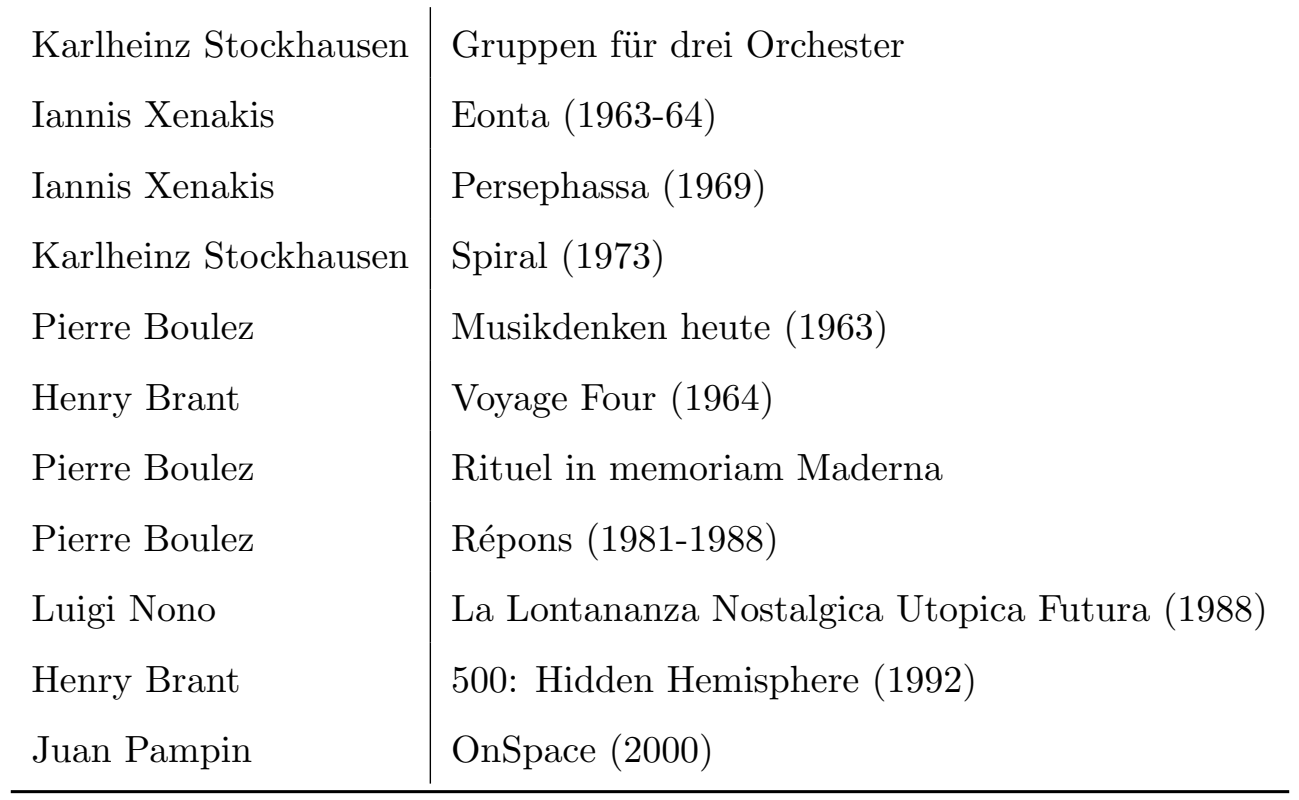

Table 2.1: Pieces with spatial preocupations.

\subsubsection{Plurifocal Music}

Several of the compositional concepts described in the context of this work were inspired by the sonic images described earlier on this chapter. From these images, the compositional process of Llorenç Barber plays an important role in the imagery of the author. Starting in 1988, Barber initiated a series of works where the entire city worked, acoustically and conceptually, as the sound resonator. Following this logic, Barber wrote scores in which each person controls the behavior of one set of bells in the bell tower of a church. Some scores were for only one church; however, most of them were for many churches within the city, opening and entirely new way of listing and living the sonic experience of the location. Over time, with the maturity of the idea, the concept of plurifocal music was coined in [27]. Barber defines three types of listening to his concerts: static audition where the public remains in a fixed location on balconies or street corners, panoramic audition, where the public leaves the center of the city and goes to the surrounding hills or lookouts, receiving the sound as a unity from afar, and 


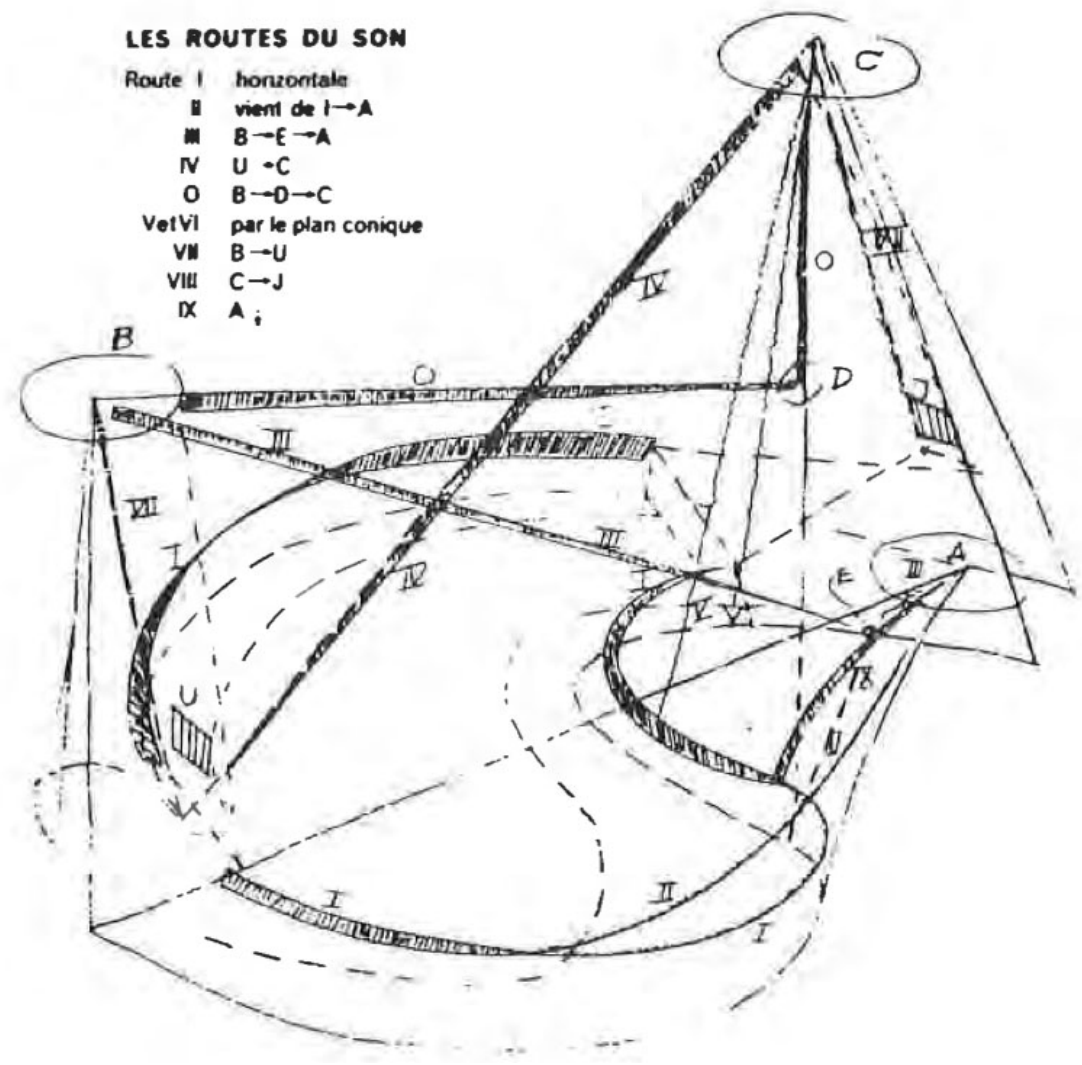

Figure 2.4: Sound paths for Poème électronique. 
peripatetic audition where the audience strolls about while listening to the echoes of sounds, immersed in the sonic tubes which are a product of the narrow streets [27].

\subsubsection{Logic of Digital Plurifocality}

While it is always possible to gather a team of musicians in order to perform physically distributed sound sources, what happens if we intend to generate algorithmic processes, hybrid works that have a digital layer of sound, or more intimate works of medium size where precise synchronization is required and we want to simplify the testing-exploringlistening loop?

Certainly putting together a set of isolated technologies to generate analog distributed sound works is always feasible, but knowing the exploration in this field was going to be long and also that several works were probably going to be produced, the best strategy was to produce a generic solution that might help to focus on the artistic process once the infrastructure was finished. With this in mind, a feedback loop between technology and sound aesthetics flourished and the term digital plurifocality emerged as a personal desire to extend the concepts of plurifocal music. The term encompass the following elements:

- Digital plurifocality extends the compositional and listening concepts of plurifocal music.

- Digital plurifocality focuses on distributed sound sources of medium and large spaces where there is an interest about exploring the relationships between the location and the propagation of the sounds.

- Digital plurifocality is mainly concerned with the use of digital elements in order to control analog sound sources in order to explore games and levels of synchronization between multiple sound sources.

- Digital plurifocality is a sonic idea supported by a specific technological solution made by the Plurifocal Events Controller and working in conjunction with the Juum software. 


\subsection{The Plurifocal Events Controller}

In order to create works based on the logic described earlier, it was necessary to design and fabricate a tool that allowed the author to test and deploy plurifocal works. The Plurifocal Events Controller is a custom electronic device that allows the generation of analog sound spread over big spaces. Objects, containers, jars, walls, windows, etc. can be struck, rubbed, brushed, or scratched with solenoids, and modulated DC motors. A kit with these electronic devices allows precise control of the mechanical equipment.The Plurifocal Events Controller was designed, built, and programmed by the author. Each module has a main microcontroller, a wireless communication module, a set of voltage regulators, and a set of input and output ports. The module works in conjunction with a power board. Together, the kit allows the control of motors and solenoids. With a computer and using a custom protocol, a collection of these modules can be controlled wirelessly in order to generate synchronized events spread over long distances. Fifty modules were fabricated during 2011 and used on several projects, Axial being one of them. Two different version have so far been created; the first version worked as a proof of concept and the current version has been used on several installations that spread analog sounds over areas of different dimensions.

\subsubsection{Motivations for Creating the Plurifocal Events Controller}

As explained earlier in this chapter, the main reason for creating the Plurifocal Event Controller was the necessity of having equipment that allows the precise control of analog sonic events spread over distances of up to 100 meters. The commercial options available did not solve the requirements or were extremely expensive. Building a custom solution ended up being the best solution, especially because it was introduced as part of the creative process.

The journey of creating the device started several years ago with the author's desire to learn electronics. The first musical approach by the author to the electronics field was the design of "GAB: Sistema de reinterpretación para pianos" [37], a small device of eight knobs and eight analog inputs that, in conjunction with custom software, allows a computer to generate variations on top of a piano improvisation. 
Several years later, the Arduino board became popular in the art community. While it is an interest concept, it is clear that the developers sacrificed the potential of the AVR microcontroller in order to make it accessible to the general public. Being frustrated about how hard it was to generate parallel time control with it (at least with the version available at that time), the author decided to get a better understanding of the use of microcontrollers.

The Swarm

In 2007, the author enrolled in a set of courses at the Computer Science department at the University of Washington. Among these courses, the author took the course "Software for Embedded Systems" with Bruce Hemingway. In this course, and in subsequent independent study courses with Hemingway, the author acquired the knowledge needed to build and program the board.

The last part of Professor Hemingway's course involved the programming of the Swarm boards in order to produce a sound experience. Developed by Bruce Hemingway, Waylon Brunette, Gaetano Borriello, and other people at the Computer Science department at University of Washington, these boards are small Linux microcomputers with wireless connections. They have a small screen and also an audio port. They have been used for several scholarly projects including soccer bots and flock audio generation.[15][16] The possibility of spreading sound over an entire building was so attractive to the author that I worked with the boards for several quarters. The author helped to embed the Pure Data audio software into the device, making sound production easier and faster on the board. A collaboration between Professor Hemingway and the author ended as a sound installation whose description can be read on the artist's web page.

The board allows the generation of digital sound and the reproduction of sound with speakers but it was not planned for controlling analog devices such as motors and solenoids that could trigger analog sound by hitting or moving physical bodies. Therefore the author began to dream of having such capabilities. 


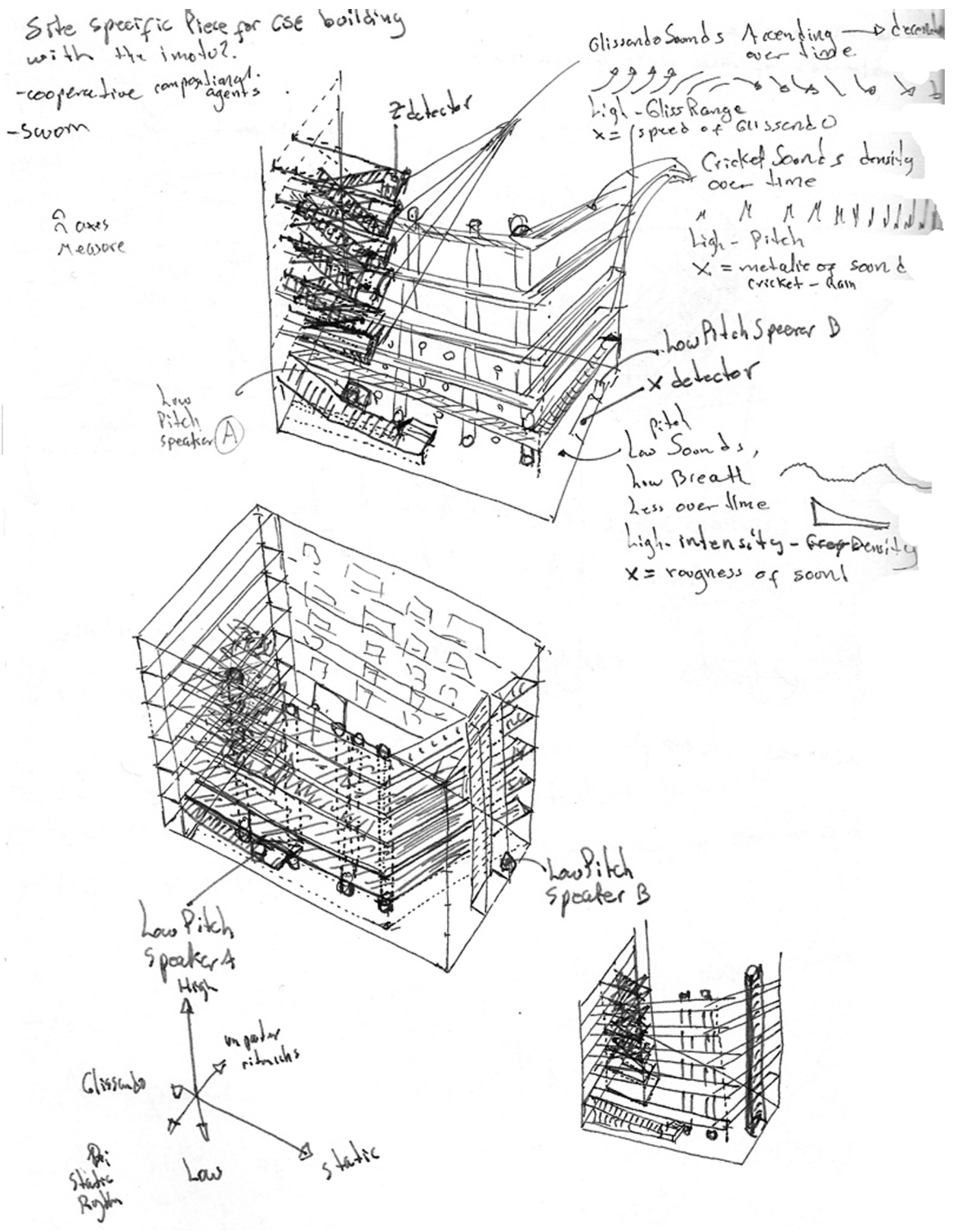

Figure 2.5: Sketch for Swarm distribution at the CSE building. 


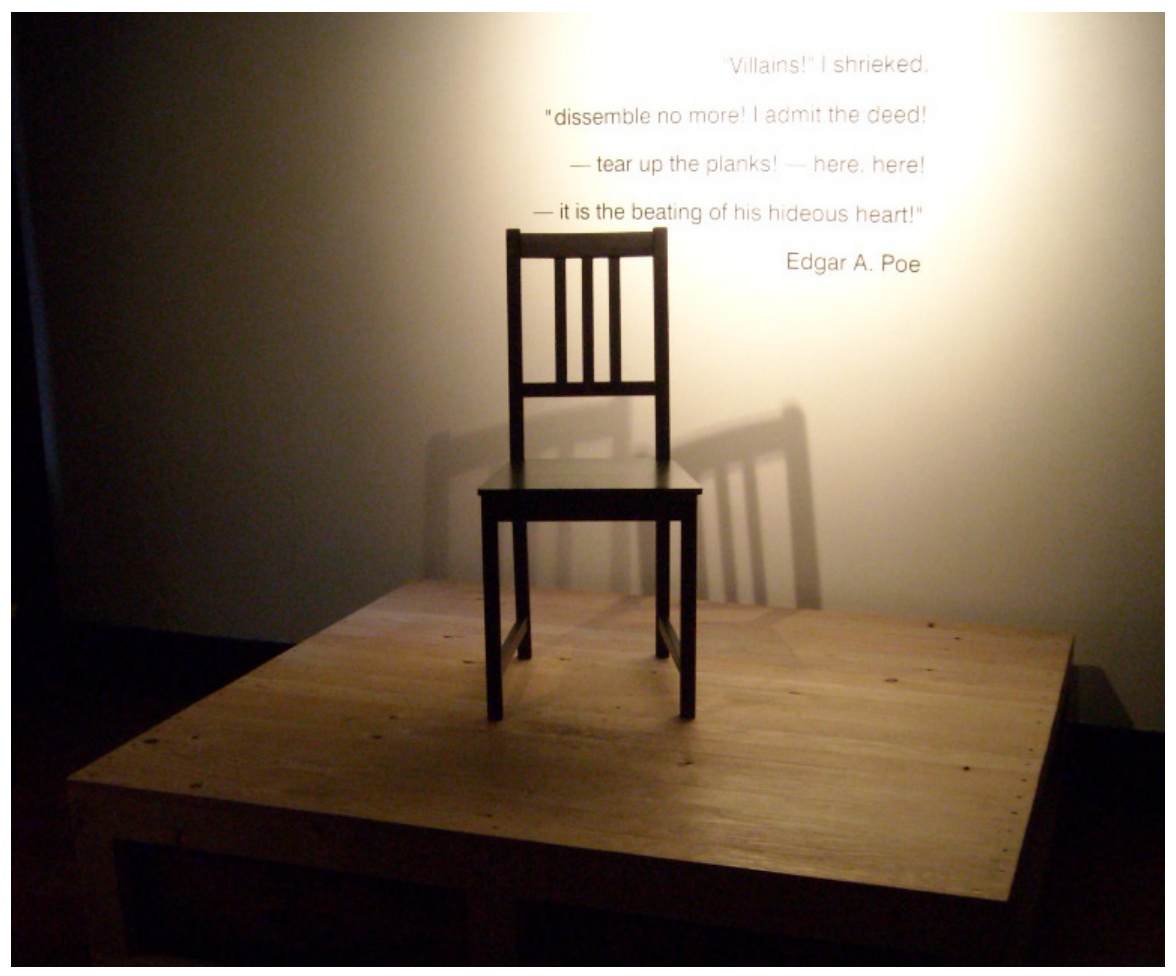

Figure 2.6: Tell-Tale Piano at the McLeod residency, 2008 version.

\section{Tell-Tale Piano}

At the beginning of 2008, the author was student in the "Mechatronic Art" course taught by professor James Coupe at DXARTS. During the course, students were encouraged to build artwork with mechanical and electronic platforms. With a piano performance background, the author was curious about exploring and treating the musical instrument as an object. With the influence of Conlon Nancarrow's work, the author worked on an art piece where several solenoids hit the strings directly. With the feedback and support of Coupe and the rest of the students, the work Tell-Tale Piano, described on the artist's web page, was finished in the spring of 2008.

In order to control the solenoids, a custom electronic board was built and programmed. The board used an AVR microcontroller a shift register circuit and a set of transistors for amplification. The piece worked fine and there were not any kind of problems during the exhibition of the work; however the electronic circuit was crafted 
in an artisan manner. The wiring took a significant amount of time, the board had to be treated with extreme care to avoid causing a cable to be unsoldered, and, more importantly, if at some point the author wanted to work with several pianos, the building process needed to be repeated again from scratch.

Knowing that situation and having a rapid-pcb-prototyping machine at the warehouse of the university, it appeared obvious that the next board should be fabricated with better and more precise technologies. Having in mind the desire to build a work for several pianos, it was also logical to work on a wireless solution. These facts lead to the fabrication of the first version of the Plurifocal Events Controller. The entire building process and the technical details are presented in Plurifocal Events Controller Specification.

\subsection{Conclusions}

This chapter presented details regarding concepts and preoccupations about sound and space. The concept of digital plurifocality was placed in its historical perspective, a set of authors and works were presented, and their influence on the author's personal work was described. Also discussed was the description, fabrication process, and use of the Plurifocal Event Controller, a piece of technology that helps to generate wireless networks for activating and controlling mechanical devices such as DC motors and solenoids. Last but not least, it was mentioned that the set of Plurifocal Event Controllers can and most of the time have been controlled with a computer. Such coordination and control have been realized with a piece of software also created by the author named Juum. This piece of software is descried in the next chapter. 
Chapter 3

\title{
JUUM SOFTWARE
}

\author{
“Tan u-juum a' ja'-a., \\ Está retumbando el río. \\ The water is sounding."
}

This chapter presents the properties and characteristics of "Juum", the software developed by the author as a personal solution for organizing and composing sonic and audiovisual artwork. The chapter presents context regarding compositional frameworks and toolkits similar to "Juum" and also technical properties of this piece of software. This chapter also explains the aesthetic and conceptual elements that led the author to develop a custom solution for his artistic work. Special attention is paid to the concept of the "art-technology feedback loop" produced when custom software is created for artworks and these created artworks are then used to produce new iterations of the software. Finally, the chapter presents some of the art projects created with "Juum" to date.

\subsection{Motivation}

If the medium permeates the meaning, and the characteristics of the medium are given by its internal configuration, then in the digital context the code that generates the medium will impact the final meaning. This is true at different levels of the analysis: technically, by defining our pallet of possibilities and constraining the resources depending on the software we employ for creating a work; socially, by subscribing the user to a particular group that employs the same tools; and pedagogically, by the influence of the previous works of the community on our work.

In a historical moment where specific code is developed for specific works of art, we can suggest that the code that generates the works is a hidden part of the works themselves. If the code changes the artwork changes, and the artwork would not exist 
if the piece of code - created with the artwork in mind - would not have been written. That said, it is possible to see the action of coding as an extension of art production. Therefore, the importance of developing our coding environments is not only technical but also aesthetic and conceptual.

In the last century, with the gradual incorporation into society of digital technologies, Wagner's idea of total art has become at least partially achieved. From superficial entertainment to sensual and original artworks, creators are exploring and creating works of art composed of several modules, each in different media. Audio, video, sculpture, painting, dynamic graphics, dance, theatre, electronics, mechanics, poetry, literature, methodological science - these are among a huge list of human activities that are now digitally interconnected and open for artistic exploration.

While working in a single media is still attractive and open to discovery and creativity, the expressive possibilities of interrelating different areas are more attractive for many creators and offer a bigger space for original and challenging expressions. Having this in mind and after a period of study in the field of computer science, the author decided to create a personal solution that integrates the different requirements that emerged constantly in the author's productions: sound generation, control of mechanical devices, access to databases, graphic control, and importantly, time coordination between all the parts.

Since the beginning of the computer era, artists have been interested in employing computer applications for creative purposes. An imposing number of pieces of code have been written in a wide variety of programming languages addressing artistic questions from a broad range of perspectives. The use of digital technologies has opened a entire world of creativity, offering a set of previously unavailable options, a sometimes misconceived potential, a way to speed up certain processes, and many other benefits, constraints, and paradigms of art production. The intersection of art and computer science has produced, among other things: pieces of code that generates materials to be executed by analog media; routines that generate pseudo-random motives or stochastic patterns, sequences, and motives; code that responds in a trivial or sophisticated manner to external analog inputs; and pieces of code that models analog representations or try to mimic human behaviors such as memory, expression, and improvisation, to mention 
just a few.

The approach has varied significantly over the years and nowadays we as digital artists coexist with a variety of solutions for art production including use of generic programming languages such as Java, Python, Ruby, C, C++, and LISP; use of free and commercial libraries for programming languages such as JSyn, pyAudio, and PortAudio; use of object-oriented programming languages such as Processing, openFrameworks, CSound, SuperCollider, PureData, and MaxMSP; and use of devoted hardware such audio samplers, sensors kits, and multimedia equipment. With such a quantity of possibilities, we face not simply the question of how to solve a particular technical solution, but which option will best allow us to focus on the artistic essence. However, the decision will inevitably shift the artistic result and as mentioned earlier the decision could be part of the artistic process.

\subsection{Digital toolkits and similar software}

A significant number of toolkits oriented toward artistic creation have been created over time. After all, a huge amount of artwork shares the same technology and same technical paradigm. Given a number of iterations of the process, it was natural to reuse the same pieces of code, leading first to the creation of APIs and libraries and later to pieces of software with a clear and defined orientation toward artistic production. "Juum" takes inspiration from several of these technologies and integrates some of their individual properties into a single framework. It may be important to mention similar technologies, inspirations, and related compositional paradigms.

\subsubsection{Field}

Field (http://openendedgroup.com/field/) is the most direct influence on "Juum"; in fact Juum was initiated at a time when Field was not yet public and it probably would not have been created otherwise. Field was created by Marc Downie over several years while a student at the MIT Media Laboratory. Downie has produced a variety of artwork using this framework and currently it is used for all of the projects realized by the OpenEndedGroup comprised of Marc Downie, Shelley Eshkar, and Paul Kaiser (http://www.openendedgroup.com). Field allows the time organization of short pieces 


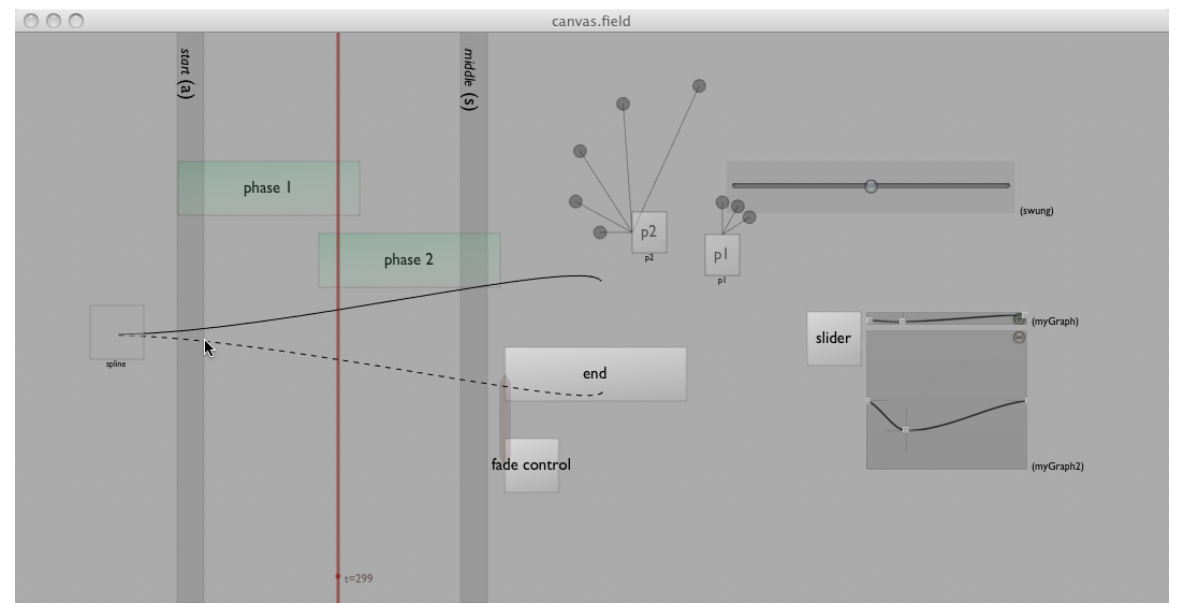

Figure 3.1: Field canvas.

of code and creates interconnected relationships between each element. Field allows the manipulation of variables through graphical objects, reducing the gap between graphical object oriented programming and written programming. In this way, Field code is the score and is the piece. Field allows the control of other programs such as Processing and Max, where time control tends to be problematic and less intuitive. The community of Field users is still small but certainly it is an extraordinary tool for creating digital artwork.

\subsubsection{MaxMsp, PureData, VVV, and other graphical software}

MaxMsp (http://cycling74.com/), PureData (http://puredata.info/), and VVV (http://vvvv.org/) are the most representative pieces of software for interactive art development based on a graphical paradigm. Easier to learn than written programming, graphical programming allows the connection of graphical objects in order to create patches that are the code and allows the modification of variables through GUI objects. While creating small structures and brief architectures on these languages is simple, producing complex and long-term architectures is, in the author's opinion, a problem and a constraint ${ }^{1}$.

\footnotetext{
${ }^{1}$ After several years of teaching and using both graphical programming and text programming, I tend to think that even though the learning curve for text programming is steeper, in the end the logic,
} 


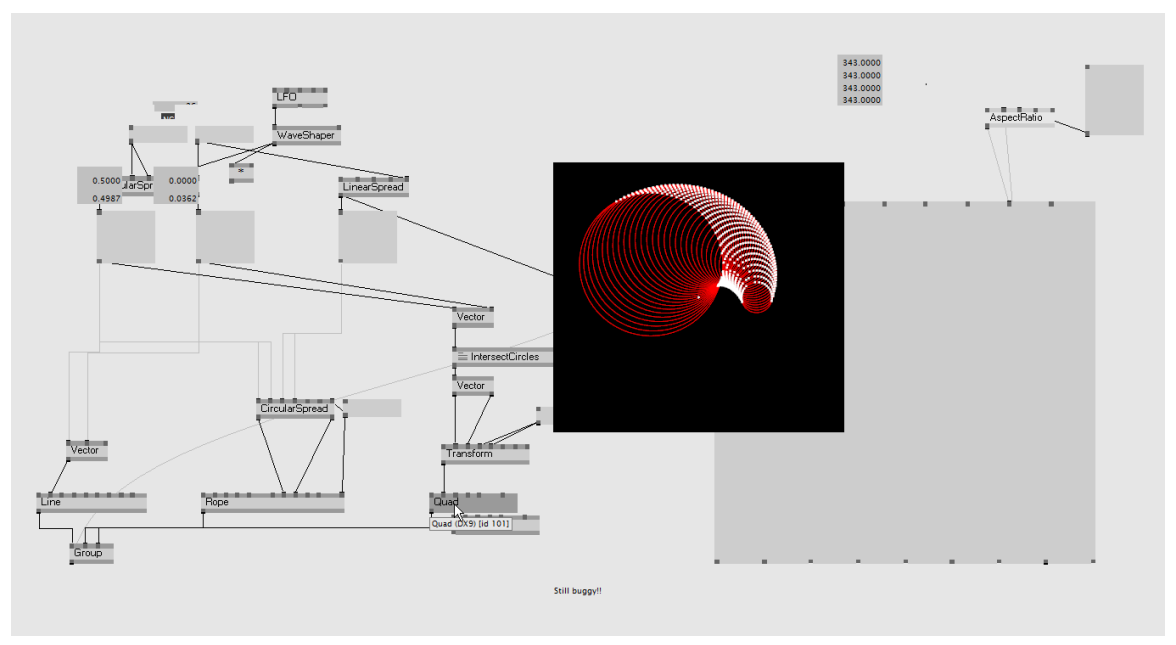

Figure 3.2: VVVV canvas.

\subsubsection{SuperCollider and other sound-oriented software}

SuperCollider (http://supercollider.sourceforge.net/) is one of the most popular, and most powerful, pieces of code for sound synthesis and manipulation. ${ }^{2}$ Started by James McCartney and maintained by a group of developers, SC is now open source code with some similarities to C Sound and other older software created specifically for sound control. SuperCollider retains the logic of chaining small unit generators in order to create structures of sound generation or transformation that are controlled in real time or by a score.

\subsubsection{Software with timelines}

The timeline paradigm is present in a wide variety of software. Traditional video and audio editors all rely on static timelines for representing fixed time evolution. On the other hand there are few pieces of software with non-linear timelines based on logical operators that can change the order of the different scenes. Having such versatility was

clarity, and understanding is much greater. For this reason the author leaves graphical programming for introductory courses only.

${ }^{2} \mathrm{~A}$ great resource for this piece of software is the SuperCollider Book[41] to which former Center for Digital Arts and Experimental Media (DXARTS) instructor Joshua Parmenter is a contributor. 


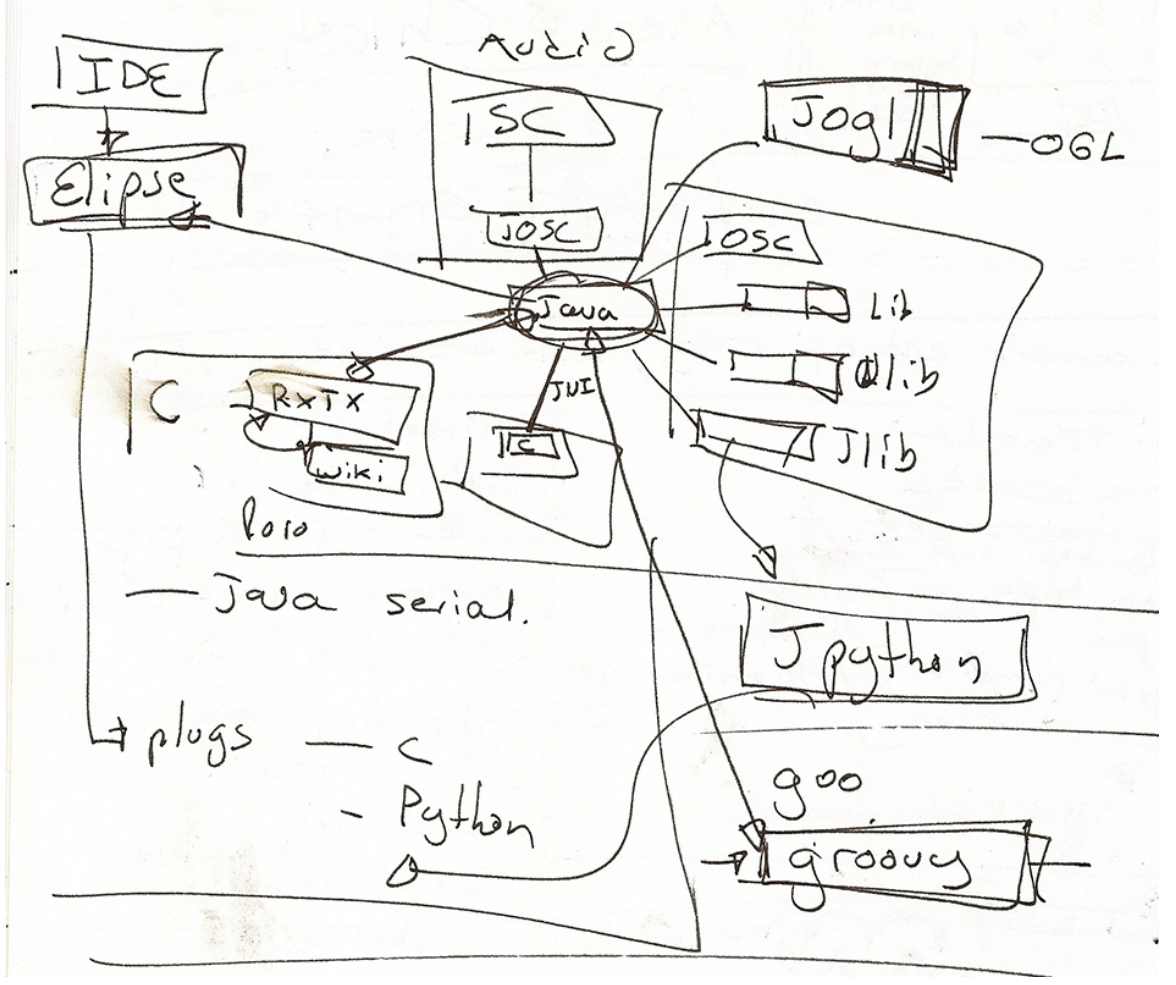

Figure 3.3: Juum initial sketch diagram.

one of the logics behind Juum.

\subsection{Description}

As mentioned before, Juum, which means noise in Maya, is a compositional framework strongly inspired by the Field software created by Marc Downie. Juum allows the easy and intuitive organization of behaviors over time. These behaviors are conceived as short pieces of code where sonic, visual, and hardware control events are algorithmically and programmatically described. Each of these behaviors (fragments of code) is encapsulated into one geometrical shape that can be manipulated into one timeline and space-field area. These pieces of code are written in a condensed scripting language, allowing for agile and dynamic programming.

These encapsulated pieces of code can be either time-based events or spatially driven events. Time events are represented as rectangular shapes and are interpreted over time 


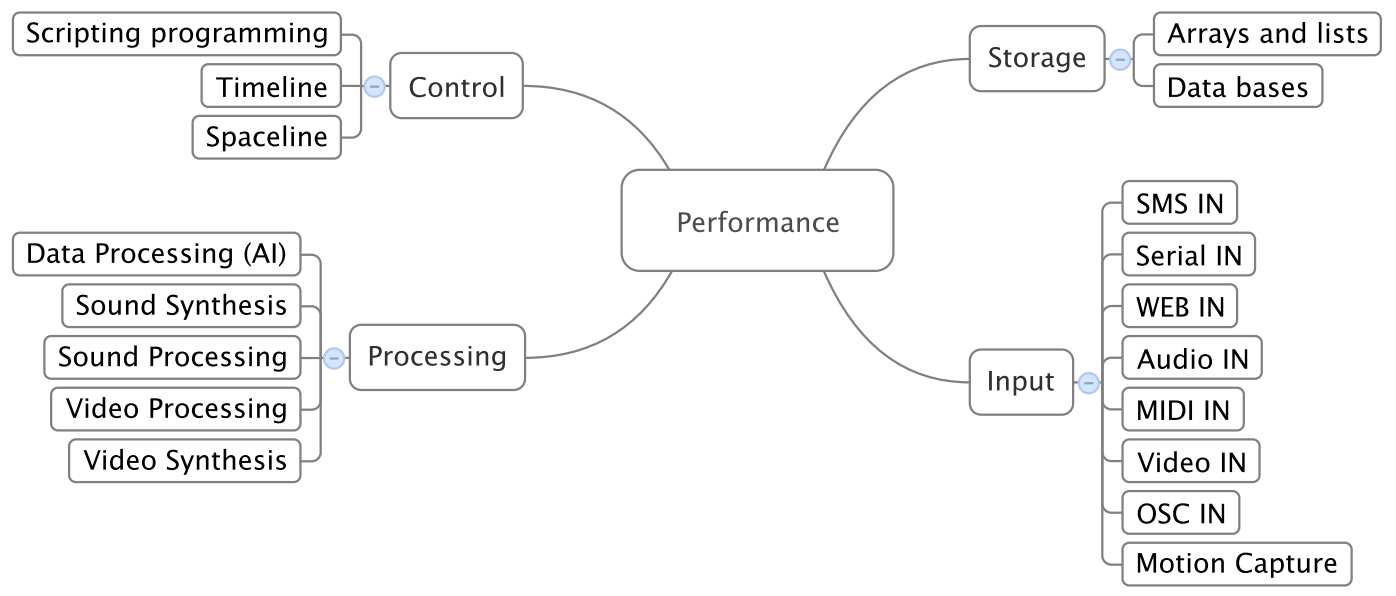

Figure 3.4: Logic of Juum software.

while the artwork evolves. Spatial events are represented as circular shapes and are interpreted along the entire duration of the work. In both cases size and location of the shape are variables and customizable in real time. Shape and location are variables that can be used as parameters inside the encapsulated code.

\subsubsection{Architecture}

Juum is written in Java and makes use of open source libraries and software, incorporated over time as new features were required. The specific capabilities and options of each library have been wrapped into prebuilt methods that are available within the Groovy console. Exposing only the methods of interest simplifies the user experience.

The materials, data, and behaviors are organized and composed by organizing the graphical elements and the corresponding Groovy code for each. The overall result is then produced in any combination of the available media and reflects any real-time modification that happens during the rendering.

The libraries and software that Juum uses internally are Groovy for the scripting parser; JOGL for the OpenGL drawing engine; Commons-Math for the statistic analysis; SuperCollider as audio engine with jCollider as SC-Java bridge; Midi Share for MIDI 
control; NetUtil for OSC control; HSQLDB as database; JMyron and OpenCV for video control; RXTXcomm for the Serial Communication; SMSlib for the cell phone messaging control; NekoHTML for the web parsing; and Substance for the GUI skin.

\subsubsection{Modules}

Juum contains several modules for retrieving, organizing, and accessing external information. It has audio, video, MIDI, OSC, serial, and video modules that allow the input, output, and transformations of several types of data. It also has methods for accessing and parsing web pages, interfacing with SMS phone messages, and parsing Motion Capture files. Juum has a database where all the data can be stored and retrieved if needed. The database is effective when working with large amounts of information that must be quickly accessed and organized. The retrieved and generated data can also be analyzed statistically to help the user to infer properties and singularities of the information.

\subsubsection{Graphical user interface}

Graphically, the software is organized into five windows. The larger, central window is the timeline and space area. This is where objects are added, edited, and moved around to organize the composition. The area works simultaneously as a timeline where a red vertical line travels from left to right during the progress of the piece, interacting with the rectangular objects, and also as a two-dimensional space where the location of the elements create spatial relationships among the circular elements.

The control window (top right) shows the script corresponding to the object currently selected in the main window. This is where the user writes all the code that has to be evaluated for that particular object. The code can be modified in real time and incorporated into the composition. Compilation problems with the geometrical object are reflected by changes in its color and contour line. This window has also the start, stop, and file management buttons, as well as options such as full screen and serial data monitor.

Juum share several properties with other programming languages that historically have been shown to be intuitive and easy to use. Juum has a console window (bottom right) where the user can read text output from the program. This is good for getting 


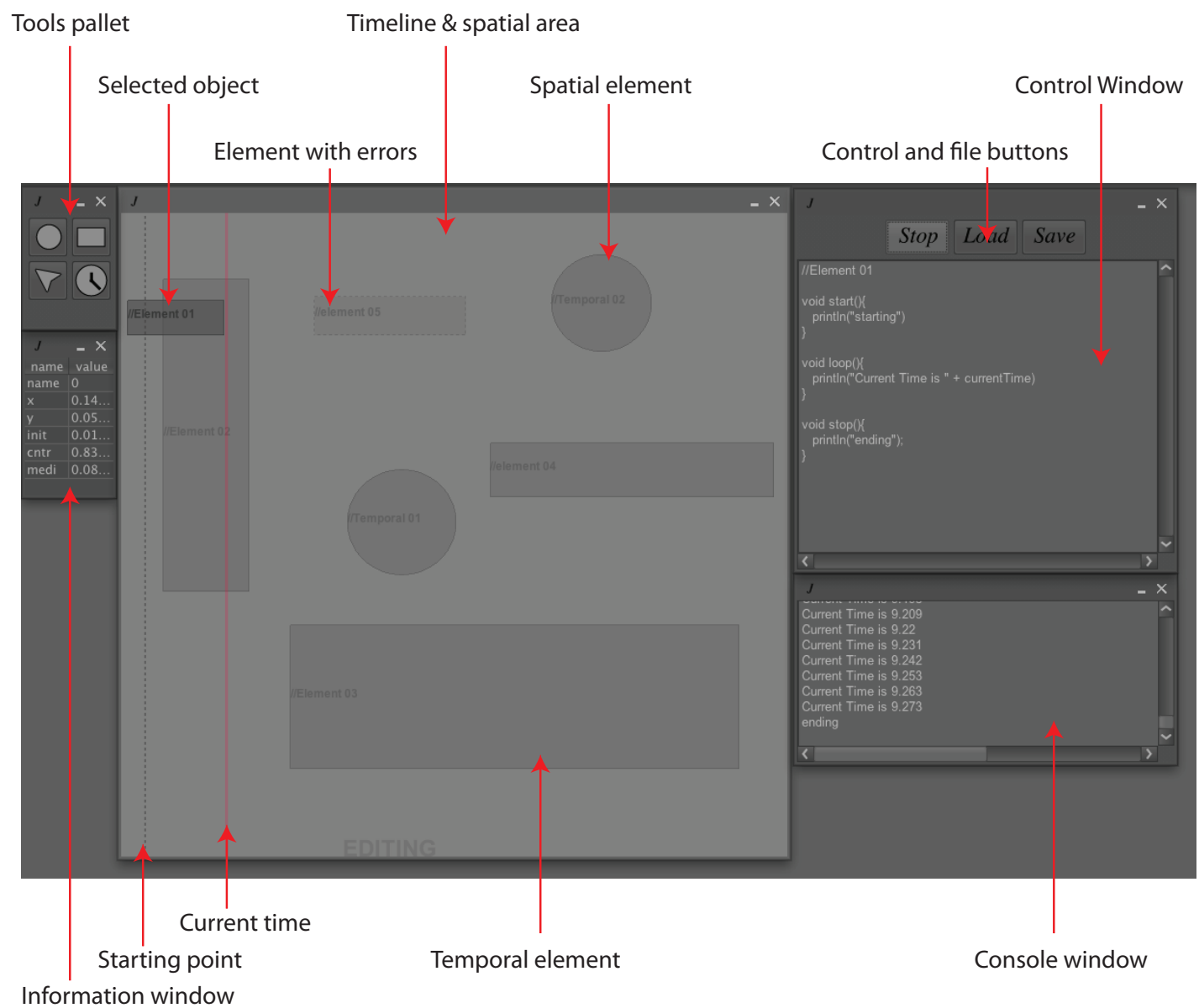

Figure 3.5: Description of windows and sections of the Juum graphical interface. 
text information from the software and also for debugging purposes. Juum has also a tools pallet window (top left) where the user selects the tool to be employed. The current version includes four tools: adding temporal elements, adding spatial elements, selecting an already created element, and changing the current moment of time. An information window (bottom left) gives details about the time/space location of each element. The numbers can be directly changed in this window and the results are reflected in the graphical objects in the main window.

If selected at launch, Juum also generates two other windows. The video window displays the video monitor and some properties of the camera such as saturation, hue, and contrast. The canvas window displays the dynamic graphics, which are shown in full screen if a second monitor is set up.

\subsection{Coding inside Juum}

Users interact with the Juum software in two ways: by laying out and distributing the geometrical objects in the timeline/space canvas, or by writing code for each of the individual graphical objects. The language used for writing the code for the objects is Groovy [21]. Groovy is a scripting language with features like closures, builders, and dynamic typing which make it agile and compact. It is an ideal option for developing short pieces of code where you do not need variable declarations or type checking. The following example shows a simple basic routine with printing elements at the start and end moments, and a variable counter that is increasing at a controlled rate inside the loop method.

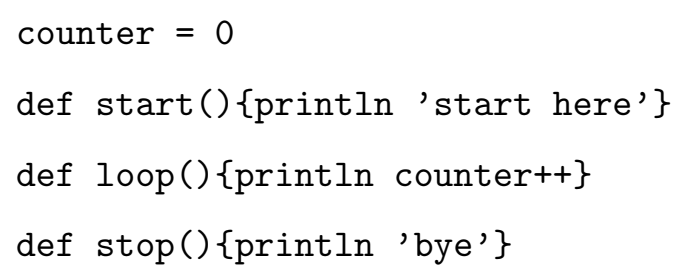

\subsection{Use of Juum in artistic contexts}

Juum has been used over the last two years in several performances and projects and it is now integral part of the authors solo improvisations for piano and electronics. Duo 
Juum, consisting of Gabriela Villa on viola and poetry and the author on electronics, also employs this tool regularly.

The first iteration of the software was developed as a project for controlling parametric curves during the Digital Sound course at DXARTS. This initial sketch had only a static control for sound events and did not have any real-time manipulation or scripting control. The MIDI input/output and the first graphics generator were included. The second version was used in the Seattle Latin American Music Festival by Duo Juum in September 2007. At that time, the software allowed the generation of basic geometrical shapes and the pitch manipulation of prerecorded samples using a MIDI keyboard.

In March 2008, a new iteration was created now including the Groovy scripting parser, statistical analysis, serial input, and direct access to SuperCollider synth definitions. The new sound module allowed a better control of the sound events. This version was used during the composition, production, and presentation of the Duo Juum's CD Vértice. With minor modifications, this version was used in the piano and electronics improvisation during a DXARTS concert at the University of Washington. For that performance, the author wore a device that senses the movement of the wrist and this body movement was used as another layer during the computational analysis.

In May 2008, the video input and a second more developed graphics generator were included for the project Just a Minute, a collaborative work for the Dance in the Digital Domain show. This artistic collaboration by Maria Cynthia, Devin McDermott, and the author explores real and metaphorical dualities between physical and graphically projected virtual stagescapes, as well as acoustic and electronic sounds.

The software was used without modifications for a piano and electronics improvisation at the Pixilerations Festival in Providence, Rhode Island, in October 2008. The following month, Conjuration of the Memory was performed by Duo Juum. For this work, the stage configuration, the walking paths of the viola player along the hall, and the location of two pianos is artistically important. The spatial graphical objects and global variables were included as technical solution for the artistic requirements of the work. This was also the first time that real-time live audio transformations were organized and distributed over long sections of the work. In April 2009, Juum was used for a piano and electronics improvisation during the DXARTS concert at the Chapel Perfor- 


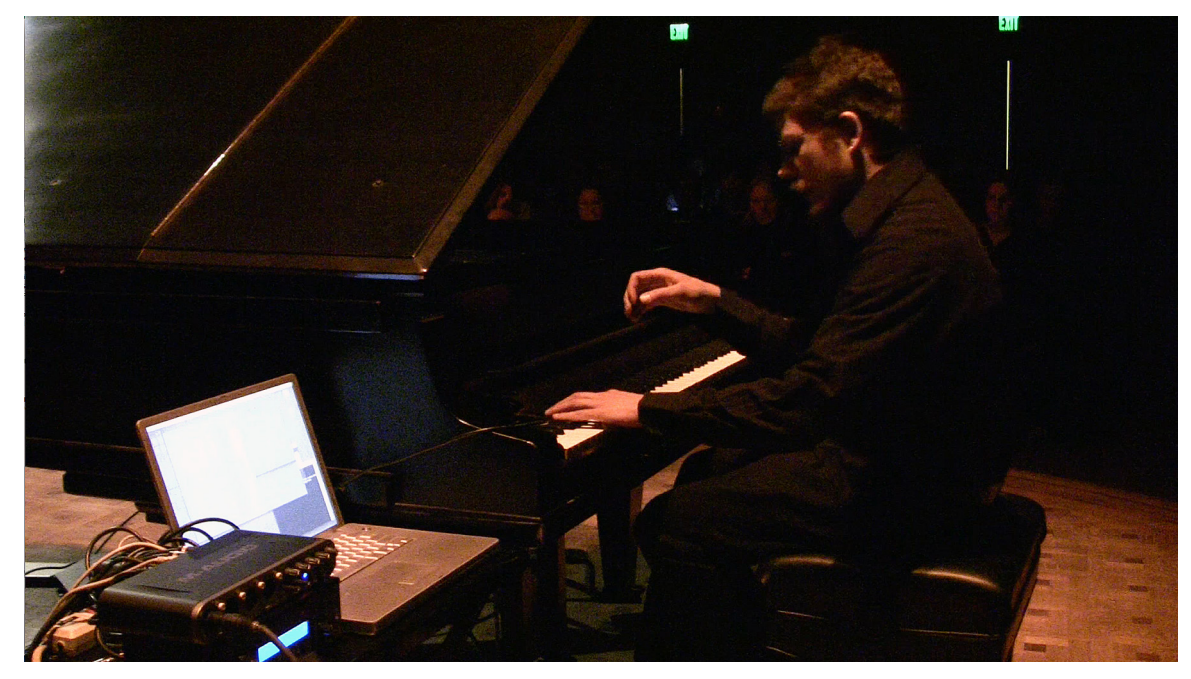

Figure 3.6: Juum software during a concert at the Chapel Performance Space, April 2009 .

mance Space. For this concert, there were no technical modifications but the software was tested with a much more complex audio layering and organization.

\subsection{Conclusions}

The characteristics, properties, and singularities of the Juum software have been presented, as well as its evolution over time and the artistic contexts where it has been used. Because of its nature, Juum will be always a work in progress. However, it is also clear that Juum benefits from the art-technology feedback loop that is occurring with the constant artistic use and updates of this framework. On the technical side, several elements need to be improved, optimized, and extended. There are also some GUI elements that are not ideal for live situations and they will be change in future versions. 
Chapter 4

ARTISTIC METHODOLOGY

"the rough sea

stretching out towards Sado

the Milky Way"

Matsuo Bashō ca. 1689

This chapter addresses the artistic concerns and questions that drove the artist's curiosity and shaped the artwork. In order to explain why Axial is as it is now, we have to do the complex experiment of introspection and analysis of the creative process. While some threads of the artwork can be explained easily and traced by clear progressive evolution of thoughts over time or by clear technical constraints and practical compromises, others are hard the track and hard to define. They belong to some images, intuitions, personal memories and evanescent dreams that belongs to the sensorial and emotional side more than the intellectual or academical paths. In any case, this chapter tries to dissect the core concepts, generators, and ideas that led to the Axial artwork, pointing to influences, historical references, or previous works of the artist as is the case.

\subsection{Artistic Questions and Concerns}

As can be seen over the previous chapters, a significant amount of research, topics, and development was applied to the creation of this work. However the modules, parts, and isolated elements should ideally constitute a piece of art, a unique and defined work that integrates all the elements into an expressive and poetic unity. The creative logic of the piece is to produce a perceptual experience, mainly sonic, using the ocean as metaphor and using the scientific research and technological development as vehicle of exploration and detonator of ideas, concepts, and materials. Several questions are intended to be answered with this artistic proposal, among them: 
- How can the sea be treated as a contemporary artistic object, with all its metaphors, historical and artistic references, and omnipresence?

- How can we use scientific research as vehicle of inspiration for artistic works?

- How can we use new technologies to produce contemporary paradigms in the artistic domain?

- How can we produce works with multiple layers of lecture and interpretation based on the interrelationship of the parts that constitute the work?

- Can we produce narratives, drama, tension, and ideally sublimation with artistic works that are not constrained to the compositional methods of previous centuries?

- Where are the boundaries between conceptual art, perceptual experience, new media, composition, and sound art?

- Can we frame technology development in such way that it becomes part of the artistic experience?

The next sections present details and artistic ideas that integrate the imagery or grammar of the artist. Some ideas are clearly observable in the artistic work, while others are subtle or even subjective detonators that are evident in the creative process but not in the result.

\subsection{Plurifocality}

Plurifocality is such a core element in the imagery of the artist that the chapter Digital Plurifocality and the Plurifocal Events Controller is entirely devoted to the definition, extension, and adaptation to the digital domain of such interpretation and conceptualization of sound. Plurifocal music relies on the distribution of sound over bigger territories, offering several types of listening and several multilayer relationships between the sound, the space, the audience, and the musical structure. Although Axial is not per se a purely plurifocal sound work, the structure of the composition and the triggered ideas are based on a plurifocal logic. 


\subsection{Public Art and Open Space}

The German philosopher Hans-Georg Gadamer theorized about art and beauty in Die Aktualität des Schönen[12]. He analyzed the aesthetic process from three perspectives: art as game, symbol, and party. For him, contemporary art requires or produces these three elements in order to generate beauty. While an explication of the nature of public art necessarily occasions an array of arguments and theoretical approximation, the author advocates for Gadamer's explanation of the expressive potential of public art in open spaces ${ }^{1}$.

Historically, at least in Western culture, the most refined artistic expressions have taken place in concert halls, museums, galleries, and collections. Secondary expressions have been relegated to public spaces: street concerts, circuses, street theater, minstrels and goliards, jugglers, and many other minor expressions. These manifestations belong to the public street, and open the possibility of generating new forms of artwork and alternative types of hybrid art.

With the slow but constant changes of the twentieth century, the paradigm has changed. The street and open spaces have gradually acquired an aesthetic value impossible to replicate in the spaces to which art has been traditionally confined. Richard Serra's steel sculptures, Banksy's graffiti, Lozano-Hemmer's public art, Christo and Jeanne-Claude's interventions, and James Turrell's concerns about light and space are just some of the many valuable expressions that cannot be explained without referencing the paradigm shift regarding the use of public space. In this line, the intersection between open space, electronic art, and interactivity has become a natural and fecund field of exploration[35][39][6][31][9].

\subsection{Journeys and Errands}

Errands have been part of human behavior since nomadic times. In some ways, "a short journey undertaken in order to deliver or collect something," as the noun is defined in the dictionary, was the first type of research and also a mechanism for expanding the knowledge of one's surroundings through exploration.

\footnotetext{
${ }^{1} \mathrm{~A}$ much more pragmatical approximation is the use of public art as a catalyst for social interaction in locations such as Mexico City, where public space is threatened by violence and lack of trust.
} 


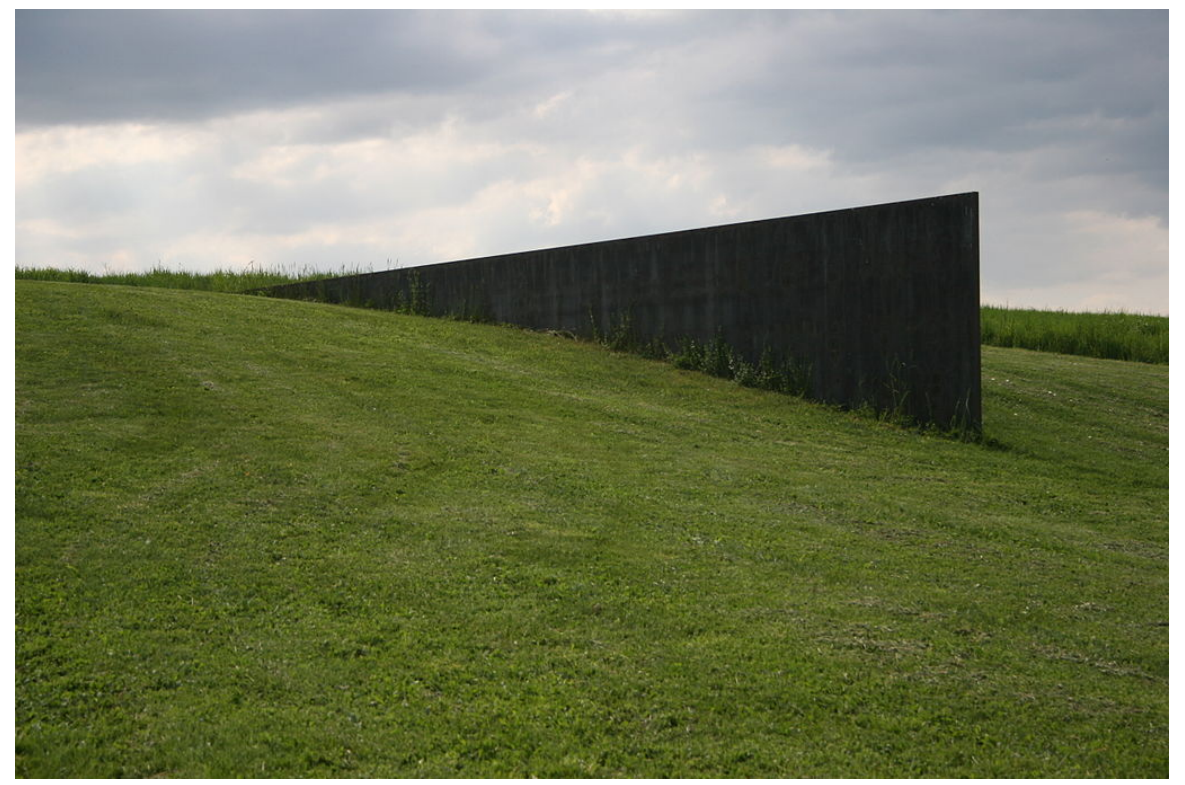

Figure 4.1: Fork2 by Richard Serra.

Also, walking was the first way of traveling and the most natural procedure to reach another location. Walking is primitive, simple, natural, and intrinsic to humans behaviors. Walking as a way of escaping, walking in order to approach somebody, some place, or someone else. Walking by surviving, by pleasure, by necessity. Walking to reach a fixed location in the shortest amount of time, walking as exercise and why not as an artistic experience.

In the first part of the twentieth century, walking was experienced as a form of anti-art. In 1921, members of the Dada movement organized a series of "visit-excursions" to banal sites within the city of Paris. This was the first time art rejected its assigned places, setting out the reclaim urban space.[3]

\subsection{Improvisation, Interactivity, and the Open Work}

Improvisation is a core element in the creative domain[7]. Comprising a broad variety and levels of use, it is present in several artistic explorations. Sometimes as a researching system during the compositional process, sometimes as final manifestation, sometimes 
with rigid set of rules and sometimes with absolute liberty, improvisation is a technique that helps to explore and search out new materials and new forms of organization. Widely used by notorious figures in art history and a core technique in some periods in history, improvisation allows artists to shape materials, regenerate ideas, and generate variations without the obligation of fixing it as a final version.

The discussion of improvisation as a technique is always an active one. Can we improvise elements that we do not master? Is improvisation as free as stated or it is only a recombination of elements in real time? Would it not be better to slaughter spontaneity in order to produce in advance a unique and final version, judged as the best version? Can improvised materials recorded on static media still be considered improvisation, even though they become rigid works? ${ }^{2}$ Will a creative state unrestrained by thoughts of the end result yield a performance with artistic merit, and does it justify the risk of not offering an ideal version?

In addition to some practical considerations, improvisation as an aesthetic is based on several precepts that need to be accepted and adopted to ensure the proper flow of the improvisational paradigm. The result is directly or indirectly, realistically or metaphorically, influenced by a feedback loop with the receiver of the work. Therefore, the material could not be compared to planned material; both situations belong to different domains of creation. While it is true that improvisation is a recombination of elements, the value of such activity relies on the fact that such recombination happens only in a particular reconfiguration with the improvisational act. It cannot happen in the same manner as other activities. Finally, improvisation, from the chaotic random version to the structured real-time composition, is also an statement about spontaneity and liberty. Ultimately, improvisation is the reflection of the fragility of life; it manifests the perishable existence and the view of living in a world in constant change without any real true. In this way, improvisation is related to the notion of the Open Work.

More than ever, the notion of having a work that needs to be finished by the spectator is conceptually paired with a society that tends to generate horizontal relationships between its members. In some way, all artistic manifestations are finished by the specta-

\footnotetext{
${ }^{2}$ The Koln concert by Keith Jarrett, for example, has been transcribed and the score performed by other pianists as a fixed composition.
} 


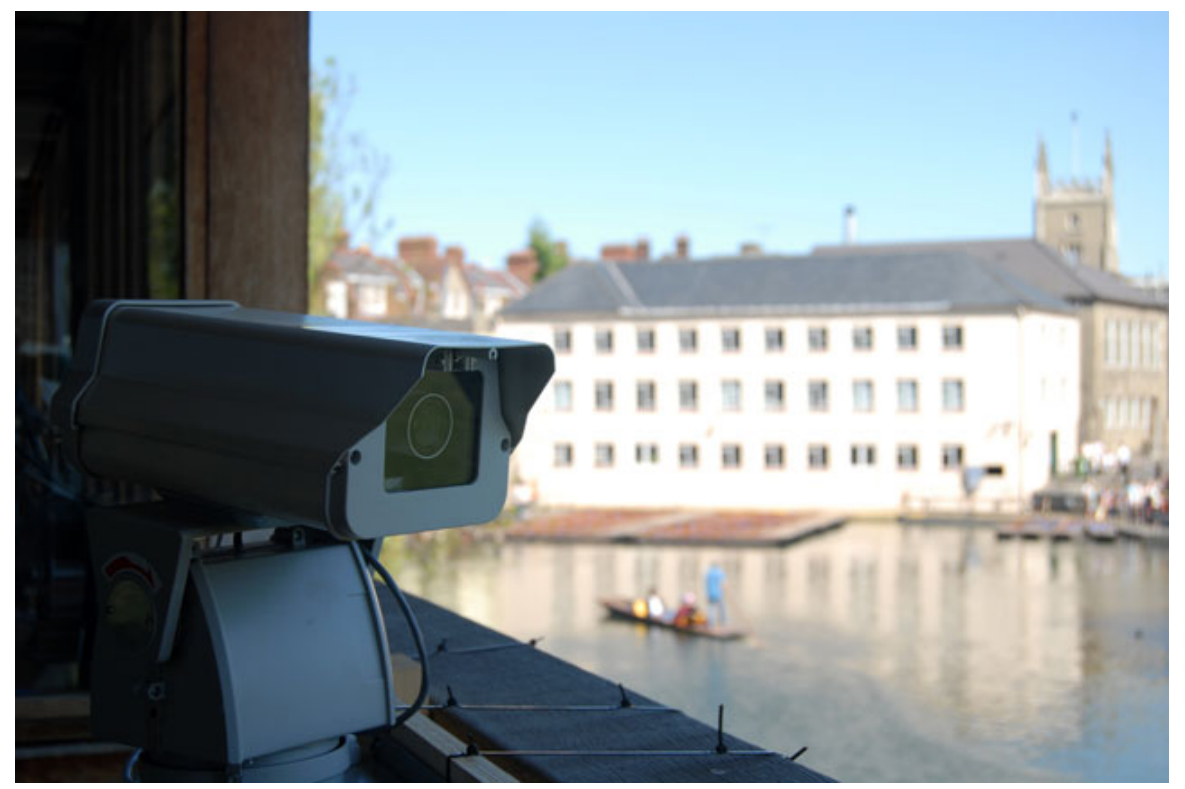

Figure 4.2: Recollector by James Coupe.

tor; therefore, all works are in some sense interactive. However, the notion of the Open Work as defined by Umberto Eco goes further by stating that "certain cultural contemporary objects participate in the common taste for instability, imprecision, complexity, transfer between other categories, and for the insistent and spatial invocation to choice and active intervention by the consumer" [10].

Improvisation as a creative mechanism has been a factor in the artist's work for many years and Axial adopted some elements of this practice in the form of algorithmic processes within the work. While the piece has a fixed structure, the interrelated sections exhibit subtle variations on each repetition. In this sense, another strong influence in the author's work is the proposal of James Coupe. Coupe takes the meaning of interactivity to a higher level with his works, in which pre-established narratives are refigured within the public space by the casual participant. 


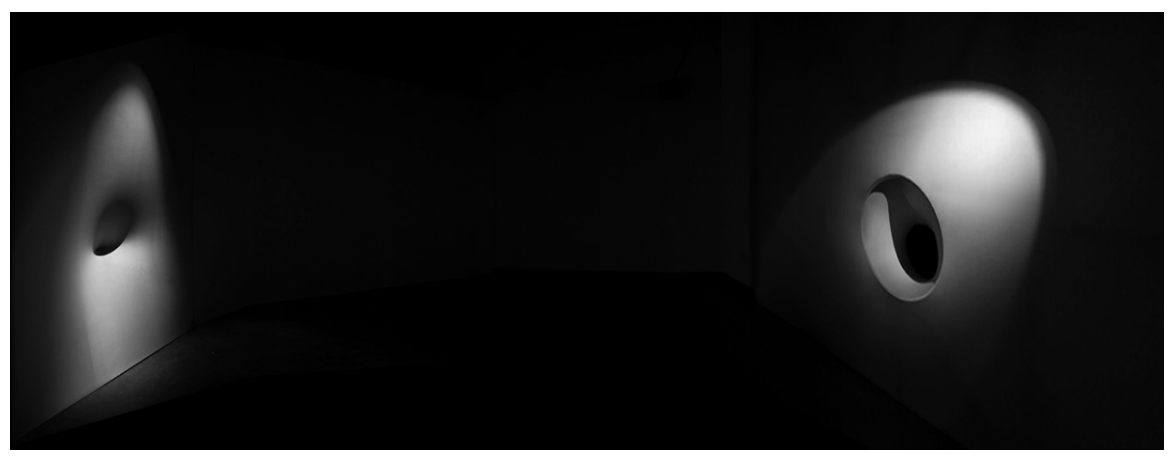

Figure 4.3: Entanglement, Juan Pampin, Eunsu Kang, and Joel S. Kollin.

\subsection{Sound Art}

The term sound art is new in music history; the definition is still not clear and is open to discussion and analysis. ${ }^{3}$ The term was coined by Dan Lander in the mid-1980s [26] with the intension of defining an artistic practice started in the 1960s. In a general sense, the term refers to an artistic practice wherein the use or evocation of sound is fundamental but different from traditional music composition. The use of time as a discursive element is diluted by the use of external references that could be conceptual, spatial, visual, or metaphorical, among other possibilities. In this way, sound art refers to expressions that bridge categories that are hard to classify with traditional terms and tend to generate hybrid intersections between sound expressions and other fields such as visual art, architecture, ephemeral art, and land art, among others[17].

The use of the term becomes even more problematic since its meaning and use tend to vary between different communities and between different languages. With a narrower definition than its English counterpart, the German word Klangkunst[22], which literally translated means "sound art," describes a practice with an aesthetics similar to that of Christina Kubisch, Hans Peter Kuhn, and Felix Hess, to name a few[33]. In this sense, the work of Juan Pampin has been of extreme importance due to his profound preoccupation with sound and space.

\footnotetext{
${ }^{3}$ The graduate seminar Sound Art at DXARTS by Juan Pampin is and advanced course where books, bibliographies, references, and works about the topic are discussed and analyzed.
} 
Artists coming from the domain of visual art have adopted the term as an expansion of their visual paradigms. They add an acoustic element to their visual proposal and explore the relationships between visual elements and sound, with a tendency to keep their focus on the visual content. Sometimes musicians with traditional background have also had the tendency to favor traditional compositional paradigms over an exploration of new relationships between time and sonic material.

Conceptual artists have entered into the field, with the result of expanding our thinking about sound and our relationship to acoustic material. The theorization of non-cochlear sound [20] is an interesting manifestation of works where the essence and inner logic tends more toward our thinking about sound than to the real acoustical phenomena.

Should we be worried about finding a precise definition of the term sound art? Perhaps we should not be extremely worried about trying to define the term, but see it as a historical opportunity to explore new possibilities for expression and perception. In coming years, the term will inevitably stabilize and define a particular artistic practice. Artist should take advantage, in the meantime, of the field's openness to exploration and personal search.

If the process of rethinking the use of sound in art started in the 1960s, the late 1990s was a period of dissemination and expansion. The logic and use of sound in nontraditional forms is now widely adopted and used in art[24][23]. In addition, the phenomena of massive music production, dissemination, and consumption are permeating the mechanisms of perception of the younger generation[29]. The crafting of sound pieces, the compositional logic, and the relationships between sound and the rest of our senses cannot be deaf to such human changes and social evolutions. It is under these conditions that the field of sound art has its full expressive potential and poetic bonhomie. Axial follows many elements of this artistic practice.

It is important to mention that, in this field, the influence and impact of the work created at the Center for Digital Arts and Experimental Media (http://www.dxarts. washington.edu/) is important for the artistic community's interest in long-term works that go beyond the logic of the gallery or the festival production. Support from an academic institution can assist in the planning and creation of experimental works, and 
as a result artistic productions can be more open to deeper exploration.

\subsection{Electroacoustic Music}

The exploration between analog sound and digital sound has spread to highly diverse levels of complexity and curiosity [4][5]. Plunderphonics, Concatenative Synthesis, and Sound Mosaicing, are a few of the artistic proposals that have emerged recently that use Machine Listening and digital sound analysis in order to generate artistic proposals.

From the experiments of Pierre Schaeffer to works by Trevor Wishart[43], Francis Dhomont, Jonty Harrison, Richard Karpen, and many others, the exploration, manipulation, transformation, and use of timbre as shaper and compositional unity has evolved and matured, producing an extraordinary timbre palette. Some of these works have a fixed and finished output in digital format to be experienced with speakers; others are hybrid works with interaction between acoustic instruments and digital counterpoints sometimes fixed and sometimes interactive. In any case the speaker is the core element of diffusion and reproduction for the electroacoustic music. If there is a spatial concern within the work, then a set of speakers is required, generating multichannel or ambisonics works ${ }^{4}$. Regarding this form of expression the work of Richard Karpen has been a strong influence on the author, especially his works that involve real-time digital processing, as in his collaborations with Gard Knox.

\subsection{The Electronic Sound of the Analog World, Paradigms, and Formats}

At this point in music history it is clear that the boundary between traditional media and new technologies is more and more diffuse, and feedback between traditional composition and digital music is more evident. As an anecdote, György Ligeti, who has few electronic works, has said that his analog work was influenced in some degree by electronic music. Xenakis, Grisey, and Murail, to mention few, are some of the composers whose musical thoughts intersect with the analog and the digital territories. Such intersections could be structural, technical, conceptual, timbrical, or a mix of all these.

Axial and other works by the artist explore obliquely the production of electronic sonorities by mechanical mediums. While it is not central to the aesthetic proposal, still

\footnotetext{
${ }^{4}$ The ambisonics technique is also a specialty and area of research at DXARTS.
} 


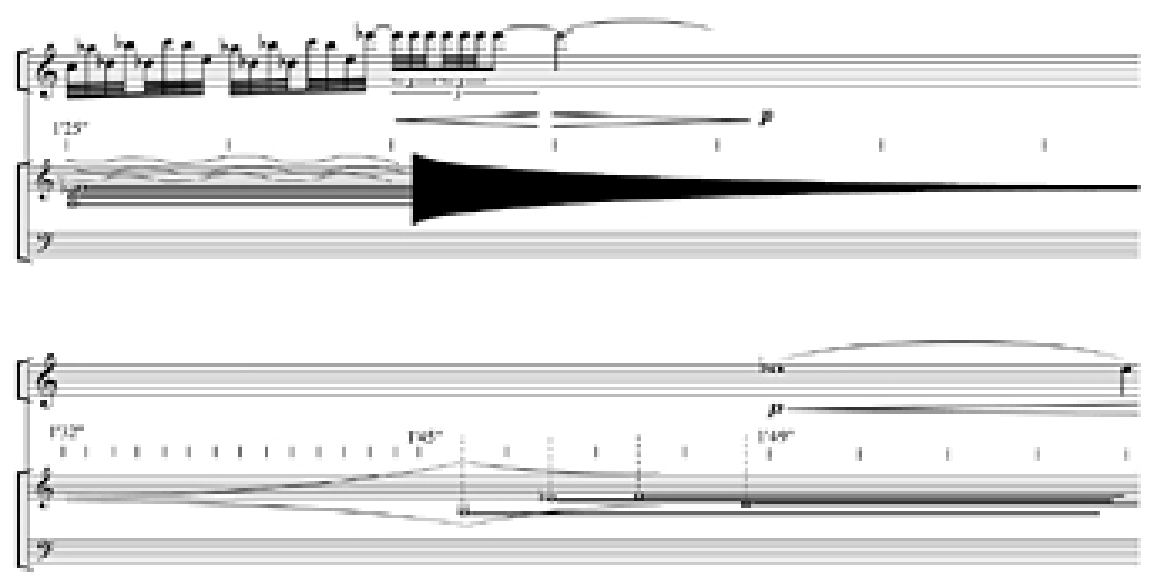

Figure 4.4: Score of "Exchange" by Richard Karpen.

it is a curiosity present in the logic. Can we generate, beyond an electroacoustic palette of timbre, an electroacoustic logic and discourse using mechanical devices? Can we use motors and solenoids to generate distributed sonorities with electronic sound properties that keep the logic of digital composition? As with other works by the artist, Axial is based on the logic of searching and exploring within the communion of digital sound, mechanical music, and electroacoustics.

\subsection{Mechanical Music}

Another field of music composition and exploration is mechanical music, which is concerned with the use of physical devices as sound generators. Such explorations could be product of a compositional curiosity as is the case of Conlon Nancarrow[13], or the result of a technocentric thought as is the case of Tod Machover, or could be coloristic as is the case of Trimpin[11], among other kinds of musical thinking. How should we list these proposals? Which musical compromises and benefits are generated by the use of mechanical objects that tend to have less timbre control but better temporal accuracy and speed?

Mechanical objects offer the possibility of generating many sound sources and spreading them over space, allowing the generation of spacial sound experiences. Multichannel digital music, and analog multi source music works different sonically, conceptually, and 


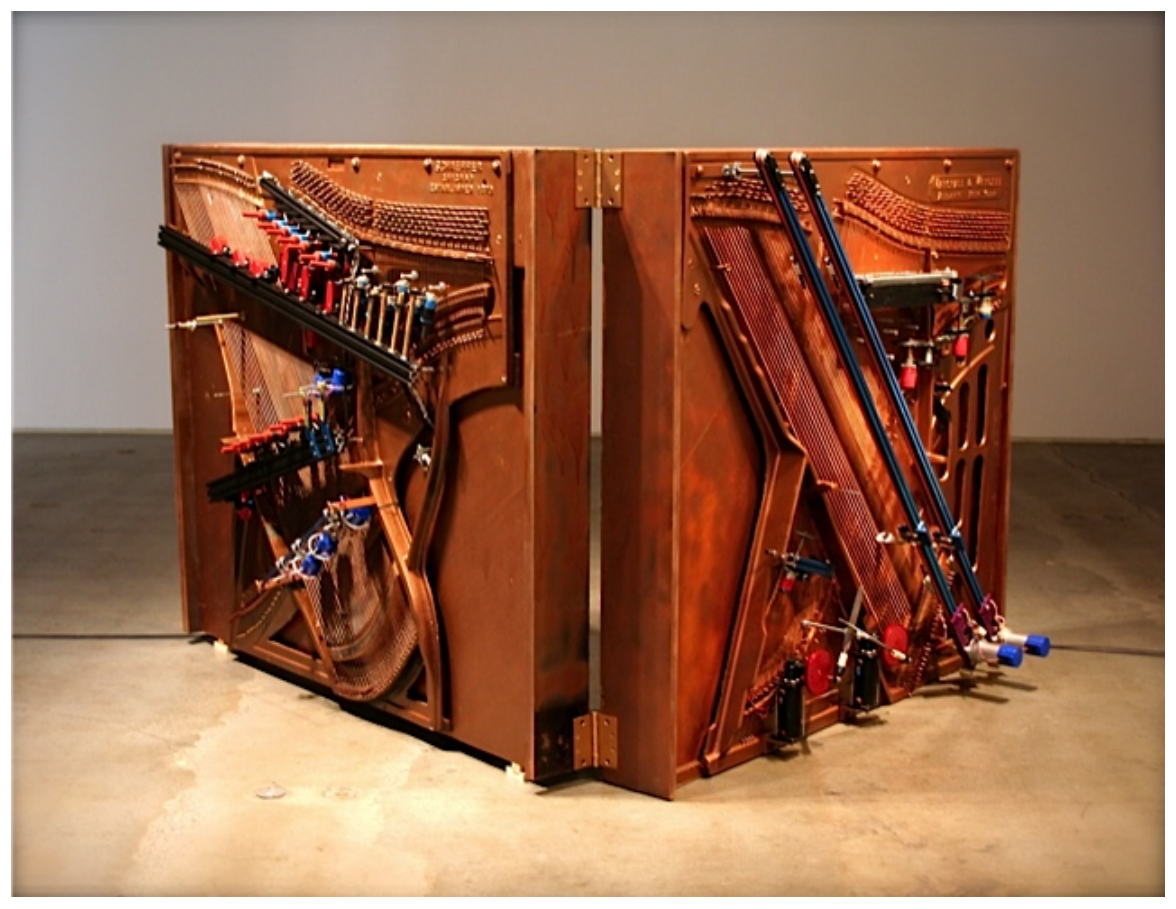

Figure 4.5: Trimpin's player piano.

physically. Each medium has its own logic with its own limits, sophistication, and constraints. Can we blend them? Can we push the limit among both domains? Can we shift the borderline between both territories compositionally, conceptually, and perceptually? Axial and other works by the author laid on this territory.

\subsection{Data Sonification, Translation, Mapping, and Modulation}

Can we extract expressive acoustical material from data that represent events in other media? It depends not only on the nature of the original data source but also on the mechanisms and decisions regarding which data sets should be applied to which sonic parameters. Moreover, the simplicity or complexity of the translation function can range from linear and evident representations of the source, to sonic materials that only indirectly evoke the original source. Linear functions make evident the source but also tend to be obvious and naive. On the other hand, complex functions may produce or help to produce sophisticated materials. The balance in the complexity of the mapping 


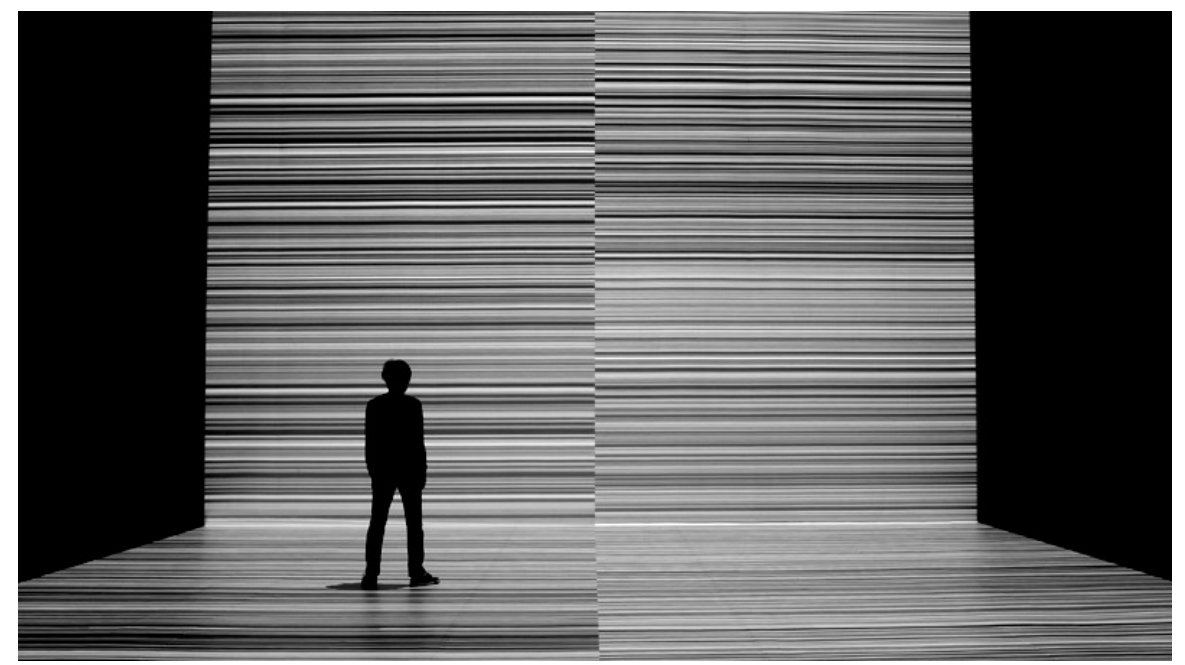

Figure 4.6: "Testpattern" by Ryoji Ikeda.

depends on the source, the handle concept, and the artistic desire.

There is always a risk involved in using functions where the source is transformed to such degree that it completely disappears from the sonic result. If that is the case, we may question the use of the original source at all, if it leaves no vestigial effect in the result. There is no simple answer to this question and there could be multiple approaches to solving it. While some people find no justification for the use of data sources that are not evident in the artistic result, for others using data as creative detonators is the only way to trigger concepts and materials to be shaped into a finished work. A deep analysis of this situation is out of the scope of this work but we can see some resemblance with the use of matrices and series in the dodecaphonic school. Are listeners able to acoustically decode the rules behind a piece? Do twelve-tone composers dogmatically follow the rules or do they bend them in some cases in favor of the musical result?

One focus in the process of creating Axial was the sonification of scientific data, specifically of oceanographic information produced by research expeditions. Prior to Axial the author had already applied, in several works, elements derived from external contexts in order to create modulations over time. Selecting the sources, applying filters for smoothing signals, choosing appropriate ranges, and finally defining a translating function becomes part of the creative process and defines the resulting material. 
Mapping can be seen as the sonification of real-time information coming from external stimuli such as light, movement, temperature, color, and position, among others. Axial is not a digitally responsive piece, one that replays external stimuli or that changes according to audience behaviors identified by some kind of sensor. Nevertheless, Axial preserves some of the responsive logic present in other works by the author.

\subsection{Science and Technology as Manifestations of Art}

Scientific and technological development are part of human evolution. Humans have created and will constantly create tools to simplify activities or leverage duties and will explore and study their physical world. The aspects of creativity and invention in science and technology are amenable to observation through artistic eyes, and can be extraordinary detonators of narrative, surprise, and drama. The intersection of art, science, and technology is certainly a fresh and fertile field of work, as can be seen in the amount of works, publications, symposiums, and courses that gradually are emerging all around the world[42]. The author is active in this field and several of his works rely on these intersections.

\subsection{Obsolescence}

An important element that the author wanted to have present in the work, at least in certain level, was the concept of obsolescence. During the first two proposals, the use of lld technology was important not as a main discourse but more as a gesture. Using old devices and refurbished equipment was important as a manifestation and label of perishable existence. It was also important as a way of give voice to the forgotten side of reality: out of fashion, out of the mainstream, away from the mass media, away from fame. That is to say, the gesture was not, at least as it was used in the first two proposals, as strong as to add artistic value to the final result. Obsolesence turned out, however, to be one of the ideas that required reevaluation during the next iterations of the work. 


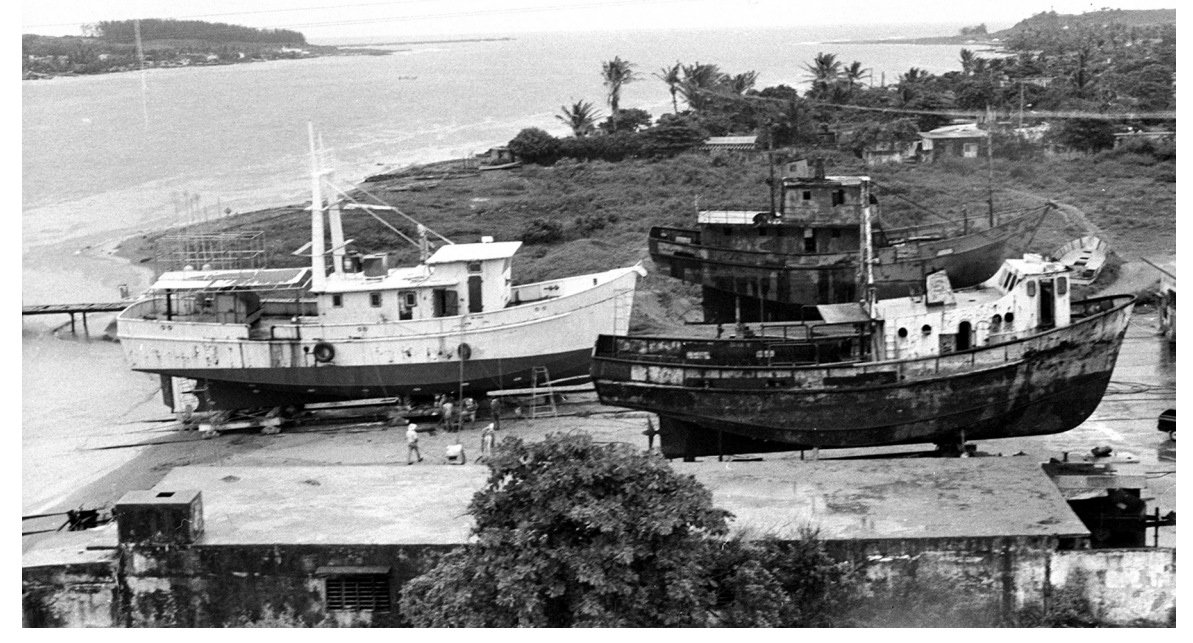

Figure 4.7: Old ships in the port of Veracruz.

\subsection{The Ocean}

The ocean is certainly a strong inspiration for art production. As discussed in previous chapters, the sea is an evocative force in human history. The sea is omnipresent and has triggered a vast amount of artwork in all imaginable formats.

The author has been exposed to much of this sea-related art production, and there is another important element that should be mentioned, which is the personal relationship between the author and the sea. The author did not grow up on the coast, but paid a few visits during childhood, and more regular visits during adolescence. Then, in 2002, the author first lived in a city near the sea. That same year the author visited the port of Veracruz and saw a pair of old boats, an image whose memory has remained strong up to the present.

Boston, Barcelona, Seattle, and Tokyo all border the sea and each has a different kind of relationship to it. Each city has a port section with cargo containers and big vessels and ships. Until this project, the sea had been a distant but delightful reference, an unknown recurrent image that had to be discovered, appropriated, and exorcised with 
a personal work. The author lives now in Mexico City where there is no sea. However, the author is starting the project Delta 16.57/-99.00 in Guerrero on the coast of the Pacific Ocean as a continuation of Axial.

\subsection{Chapter conclusion}

This chapter presented the logic behind the Axial work. It presented the questions and concerns that motivated the creation of the work as well as the concepts, references, and fields that constituted the artwork. This chapter aimed to explain the personal and subjective images that triggered the production of the artwork that will be presented over the next chapters. 
Chapter 5

\section{PRELIMINARY WORK}

"If I had eight hours to chop down a tree,
I would spend six sharpening my axe."

Abraham Lincoln

This chapter presents the process of composing the work Axial. It describes the artistic explorations, questions, concerns, and decision making which led to the finished concept. Since Axial is made of several layers, this chapter presents the concerns and development of each of these layers and each step of the compositional process. In addition, since this work was conceived with several manifestations and exhibition formats, the sketches and alternatives are also explained.

This chapter presents the reasons for the piece, the decisions made as the piece was constructed, and also the nature and logic of the incarnation of the work. This section addresses the compositional process of the final version from several approaches including conceptual, artistic, sonic, aesthetic, and technical among others. The chapter explains why Axial is as it is and how it works artistically speaking.

\subsection{Explorations and Sketches}

Art creation and probably all fields of human creativity are rarely a linear and straight path of exploration. Usually human discovery, especially art, tends to be a sinuous path with changes of direction, updates of ideas, and incorporation of discoveries at each step of development.

The logic of Axial was clear from the beginning but the process of realization experienced several changes. During the creative process, it was not clear which would be the best frame for showing the concept. Therefore several alternatives were explored over several months, based on the skills learned during the doctorate program. 


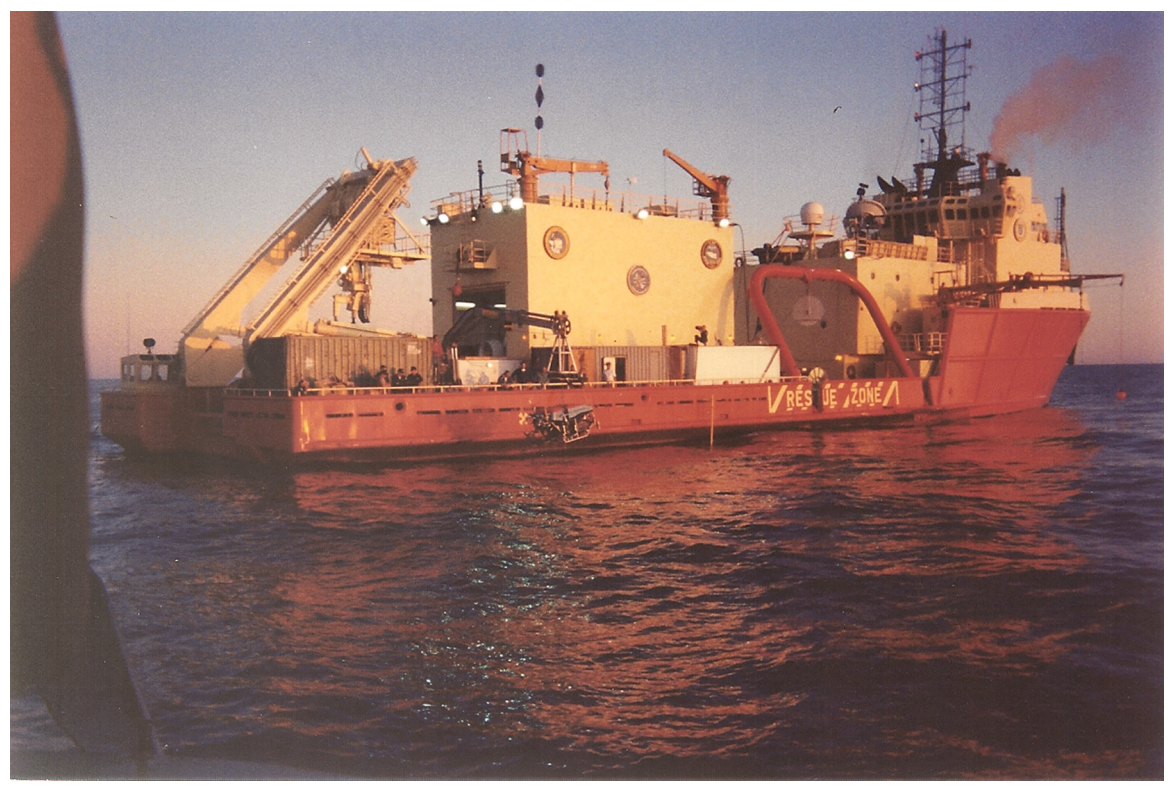

Figure 5.1: Trip expedition as part of the Jason Project in 1993.

\subsubsection{Jason Expedition}

While a high school student at the CCH UNAM, the author had the opportunity to participate in the Jason Project (http://www. jason.org/), a U.S. middle school science program started in 1989 by Dr. Robert Ballard with the goal of encouraging students to explore the fields of science and technology. Every year the Jason Project developed an expedition organized around a particular scientific interest. In 1993 the expedition was to the Gulf of California to study the hydrothermal vents at the Bay of Conception.

Students spent a week developing a research project with the support of the scientific crew at the same time that they explored and learned about the oceanographic equipment. During that period, the author assisted the ROV team and participated in observations of the hydrothermal vents.

It was during that same time that the author started to be interested in music composition. The images, sounds, and overall experience of the ship and exploration of the seafloor were approached not only from a scientific point of view but also as a trigger for artistic images. It took almost 20 years to materialize this imagery as artwork. The 
experience of the Jason Project was influential at the subconscious level for the Axial project and reminiscences of that primary exploration are observable in the work.

\subsubsection{Sea Journey at the Wood Ship}

The initial idea of Axial started during the Mechatronic Art course thought by James Coupe. During this course, the author tried to generate a sonic experience in a wood ship. The author visited the fishing port in Seattle and, by asking owners and drivers, got in touch with a Native American driver who was willing to rent an old wood ship. The driver invited the author to see the boat at Bainbridge Island and narrated several Native American histories and legends which had potential to become narrative lines for the project. Negotiations did not continue but the idea of creating a sonic journey became stronger ${ }^{1}$. The original proposal presented during the mechatronic course was the following:

The motivation behind The Voice of The Ship is to generate an original sonic experience where the object that "sings" is a ship. Customized software and a piece of hardware will be created for generating this experience. The flowchart of the work is as follows: A collection of contact microphones will be set in different resonating structures of a ship. A solenoid will be placed close to each microphone, enforcing the generation of sound. Using the software, the "player" will control the activity of the solenoids and also the transformations of the signal coming from the microphones. The "composition" will be broadcast in two speakers located on the deck of the ship. A team of sound artists will be invited to produce material that will be performed during a short trip on the high seas, where the passengers will simultaneosly be listeners.

\subsubsection{Exhalation-Water}

Exhalation-Water was the artistic part of the qualifying exam for the doctoral program in Digital Arts and Experimental Media. Knowing the author's interest in developing a

\footnotetext{
${ }^{1}$ Rheinklä nge by Christina Kubisch follows the same logic
} 


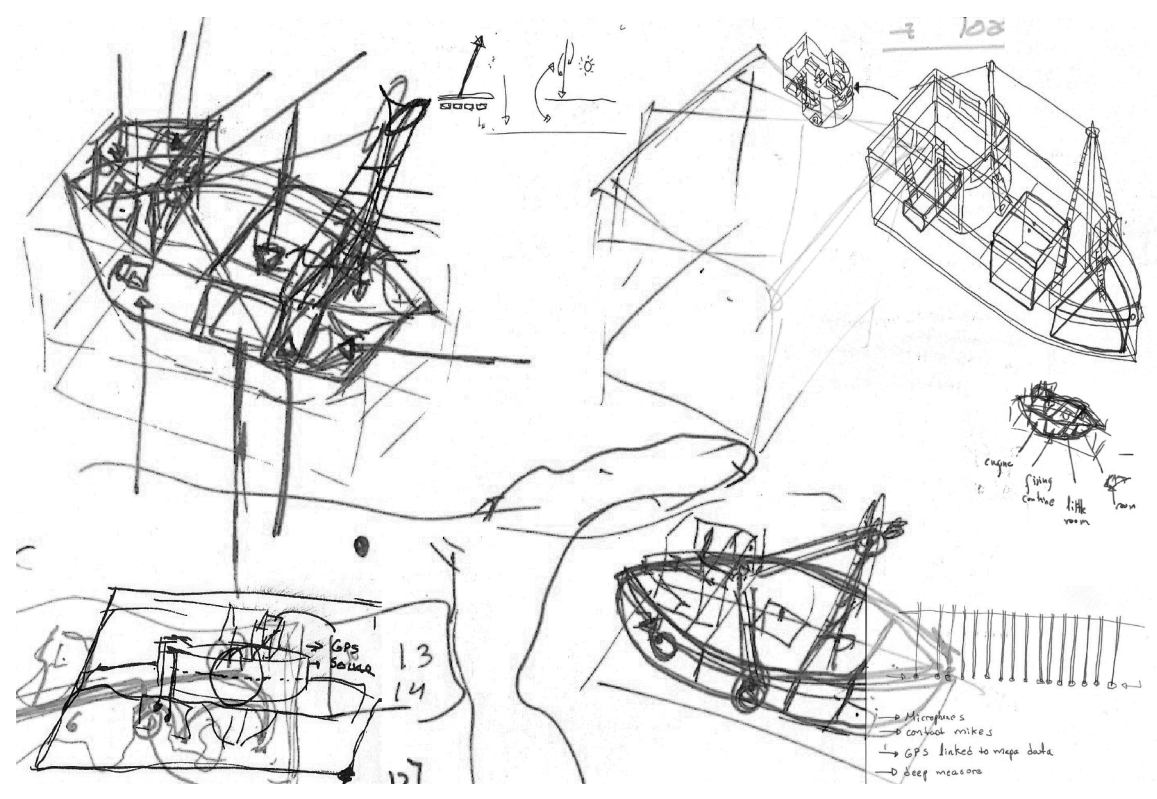

Figure 5.2: First sketches for the sea journey.

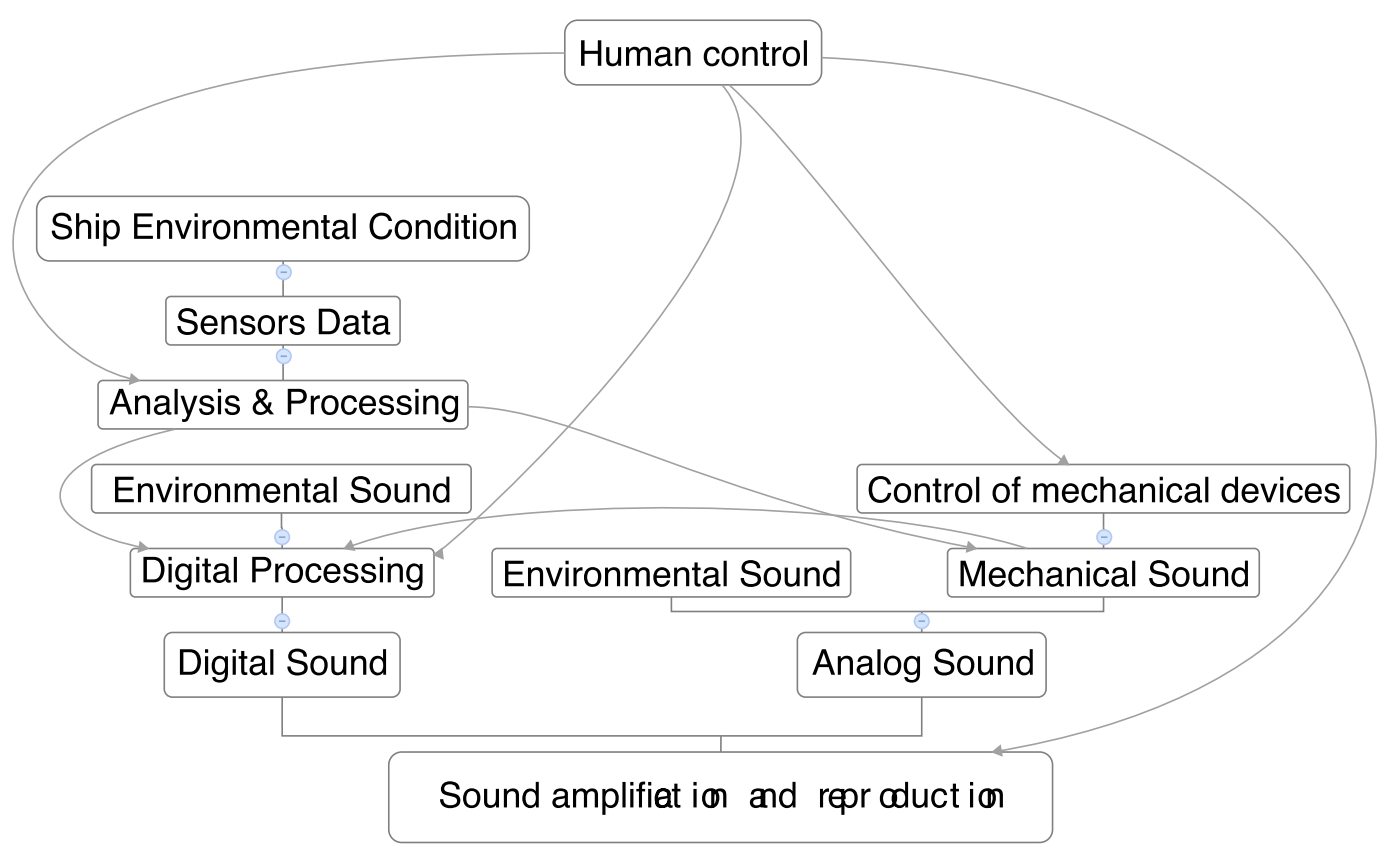

Figure 5.3: Structure scheme of sea journey developed during the Mechatronics Art course. 


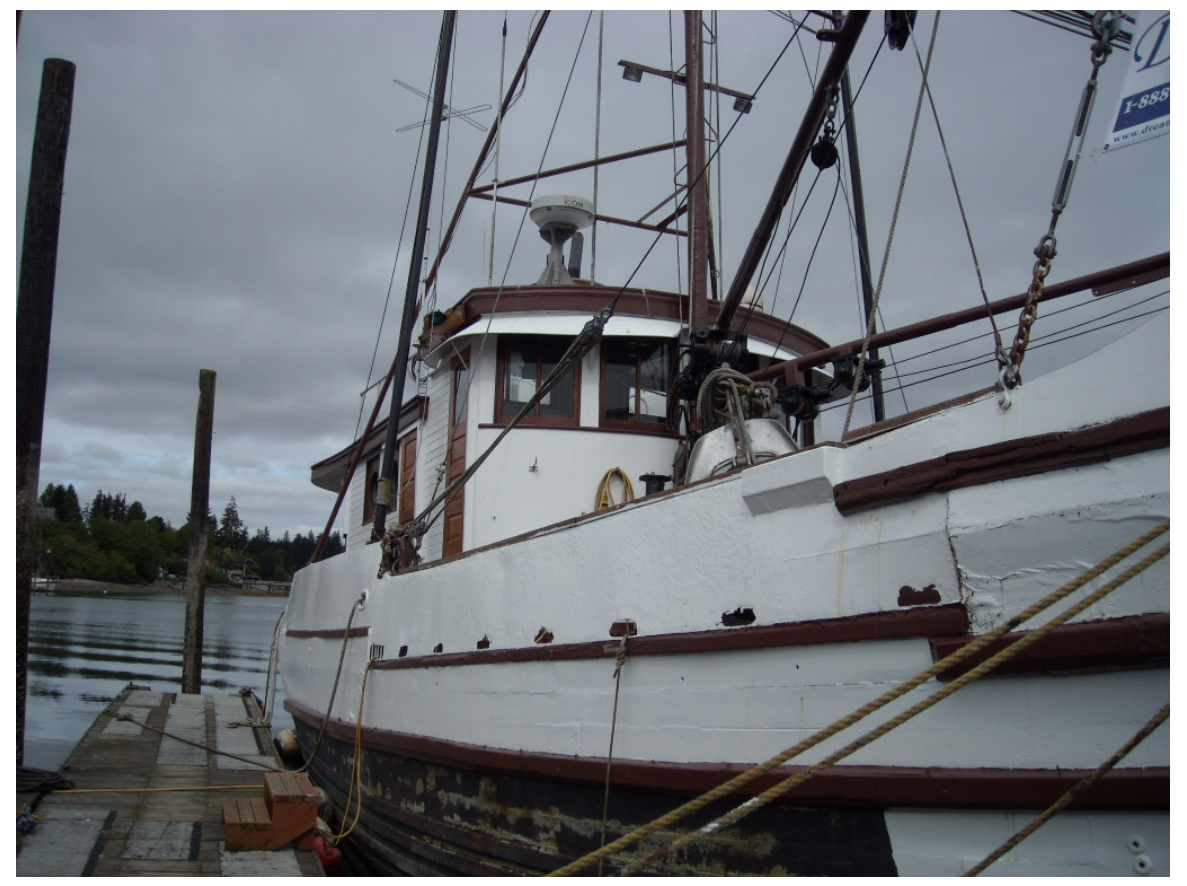

Figure 5.4: The Silver Wave halibut schooner.

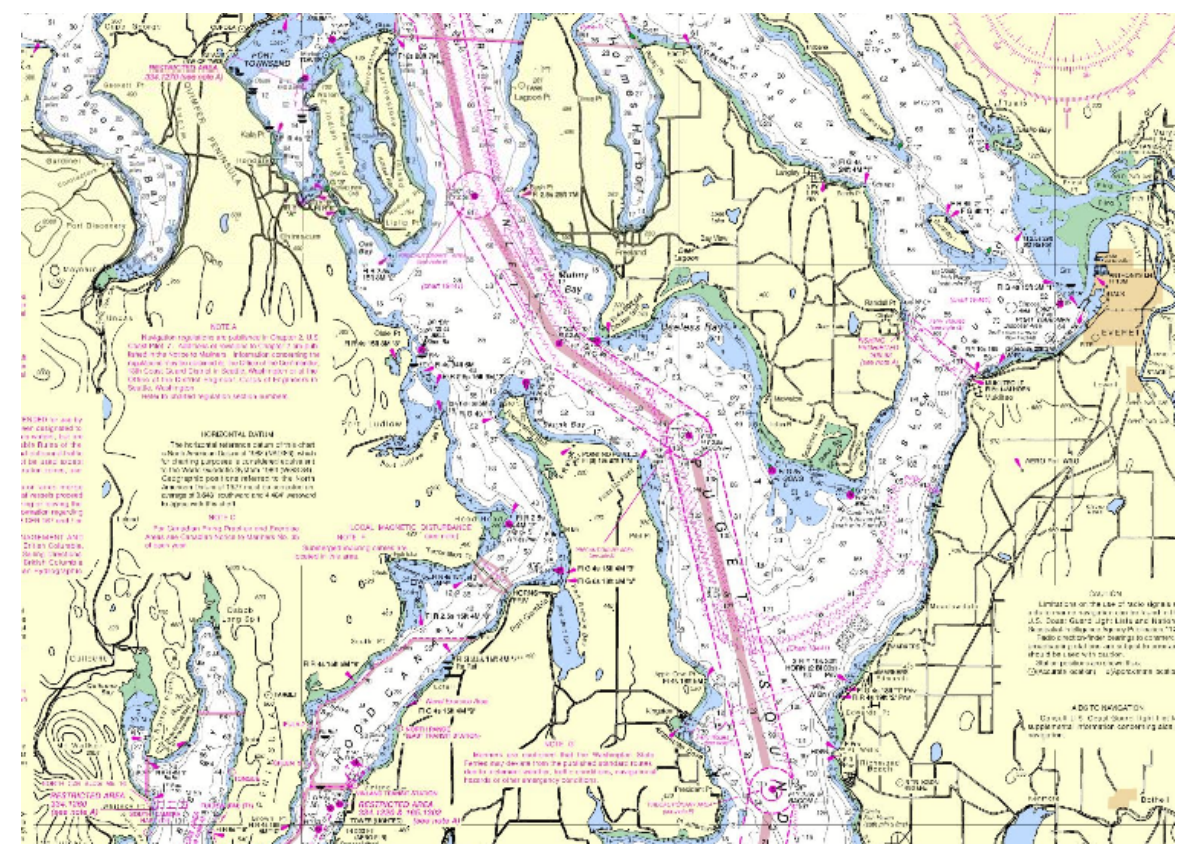

Figure 5.5: Puget sound nautical chart for planning the sonic journey. 
sonic journey, the committee formulated the following question:

Create a piece that can dynamically adapt to different environments using sound as its main medium of communication (not necessarily just through audio, other means of sound production could also be used). You are asked to set up two working instances of your piece in two on-campus sites of your choice. These two instances of your piece should absorb as much information as possible from their environment, reflecting salient aspects of the sites. Finally, the two sites should be stops on a walk you will take the committee on, starting and ending in Raitt Hall. This on-campus walk, not unlike a cinematic experience, should be a time-based poetic journey in which the two sites are integrated, having sound as its central component.

While you should be completely in charge of the production of this project, you are welcome to ask for help to deal with some of its logistics, especially moving equipment to the sites and setting things up (you might also want to have people helping you during the walk, supervising equipment left in the sites, etc.). You should also have a small budget for electronic components or other things needed to complete your project.

As a response to the question, the author designed a sonic experience that generates a dialog between the space, the sound, the environment and the movement not only of the participants but also of the sonic components all along the location where the experience happened. The details about the piece can be seen on the artist's web page. Exhalation-Water has several logical threads that continued throughout the conceptualization, development, and realization of Axial. In spite of the fact that the final version of Axial is not a journey in the sense that there is not a physical displacement of audience during the presentation experience, the idea of traveling, displacement, and movement remains a part of the presentation of the piece. The journey as a metaphorical and conceptual idea started in Exhalation-Water and remains present in Axial. In this sense, Axial is a shadow and a transfiguration of the journeys the author realized aboard the Thompson vessel. 


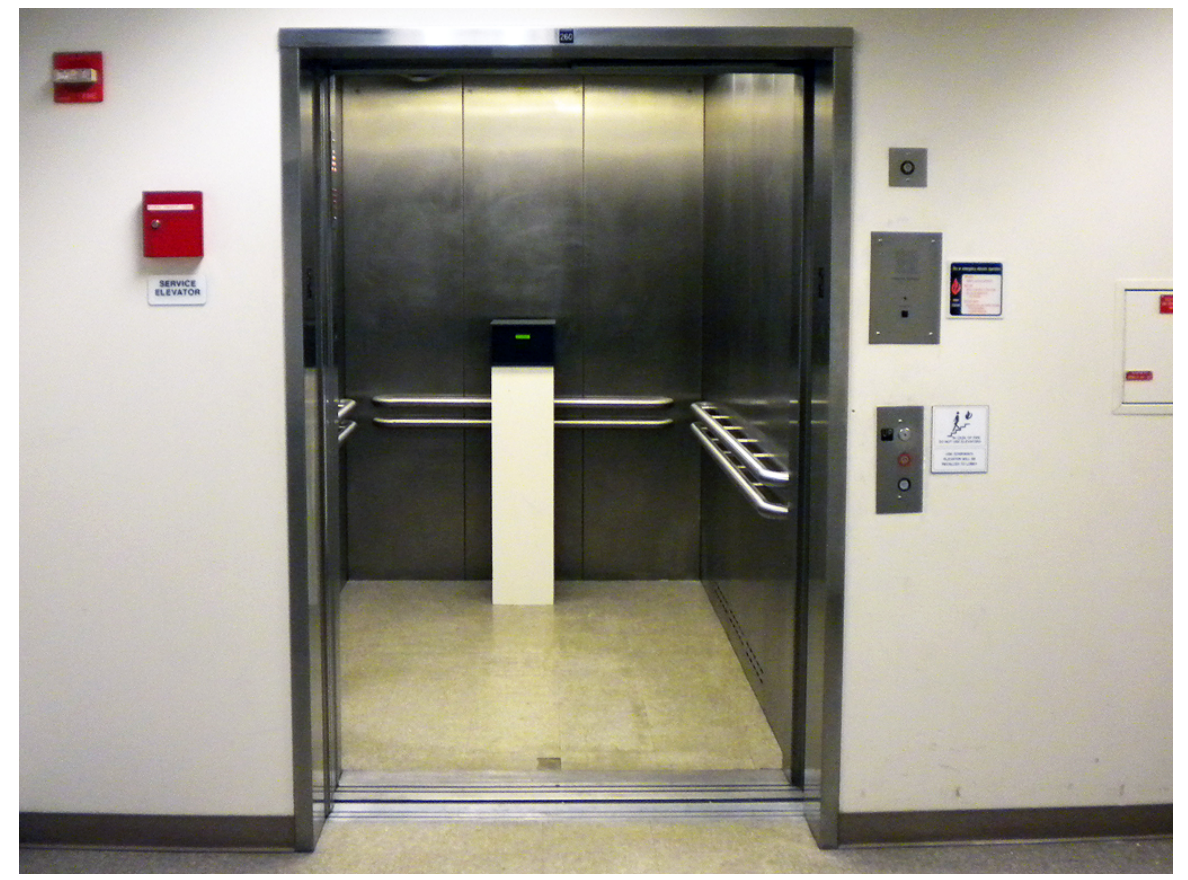

Figure 5.6: Exhalation-Water's audio analysis box inside an elevator at UW.

\subsubsection{First Expedition, Imagery}

During the summer of 2009, the author took part in the Lumens expedition. However the expedition got only as far as the Ballard Locks before the R/V Thompson experienced serious mechanical problems and the cruise was canceled.

Although the expedition was canceled, students spent a couple of full days aboard the vessel and a set of lectures and talks took place on the ship. During this period, the author did a set of sound recordings and also an intense photo documentation that was important as a visual mental reference. While not scientific, the acoustic, spacial, and sensorial relationships between the Thompson, the Ocean Observatory Initiative, and the author started during this trip. Walking, listening, and understanding the evocative images of the location helped to have a much better idea about how to approach the experience for the next expedition. 


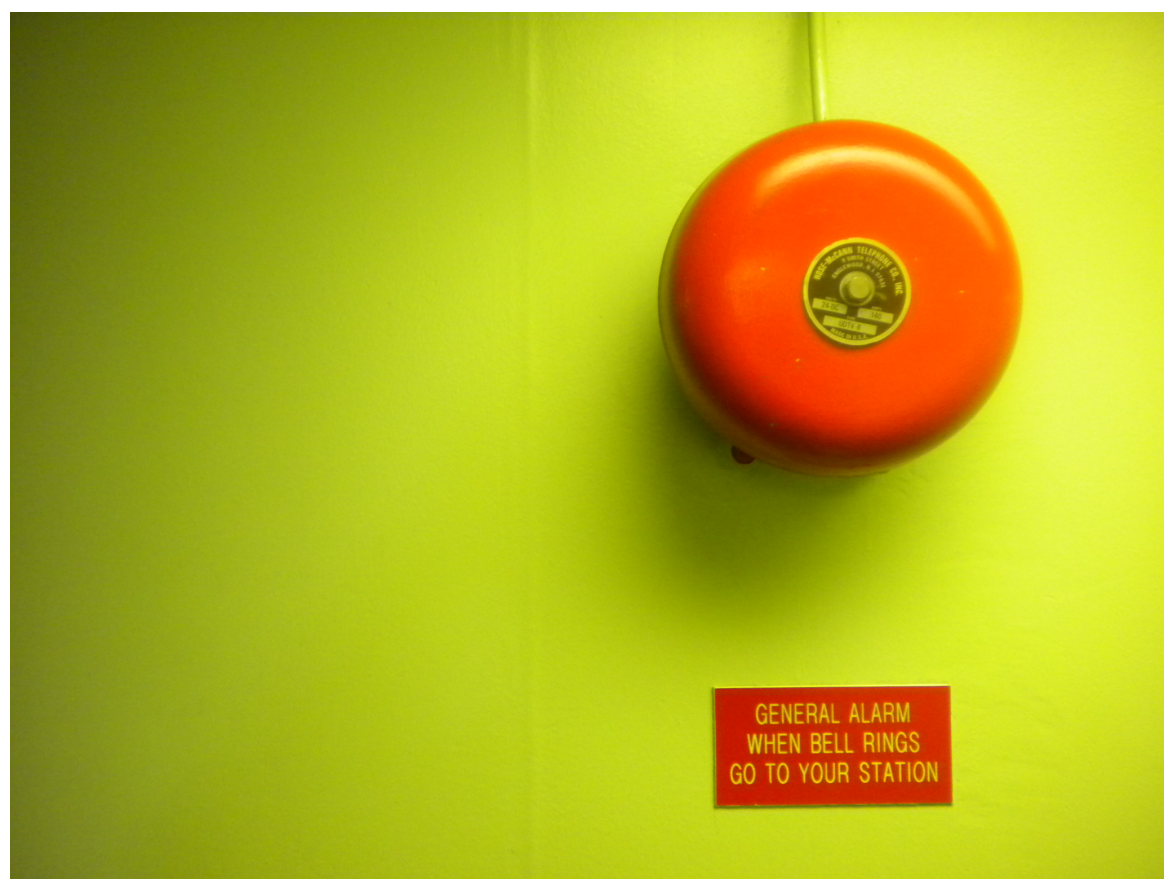

Figure 5.7: Picture taken during the Lumens expedition.

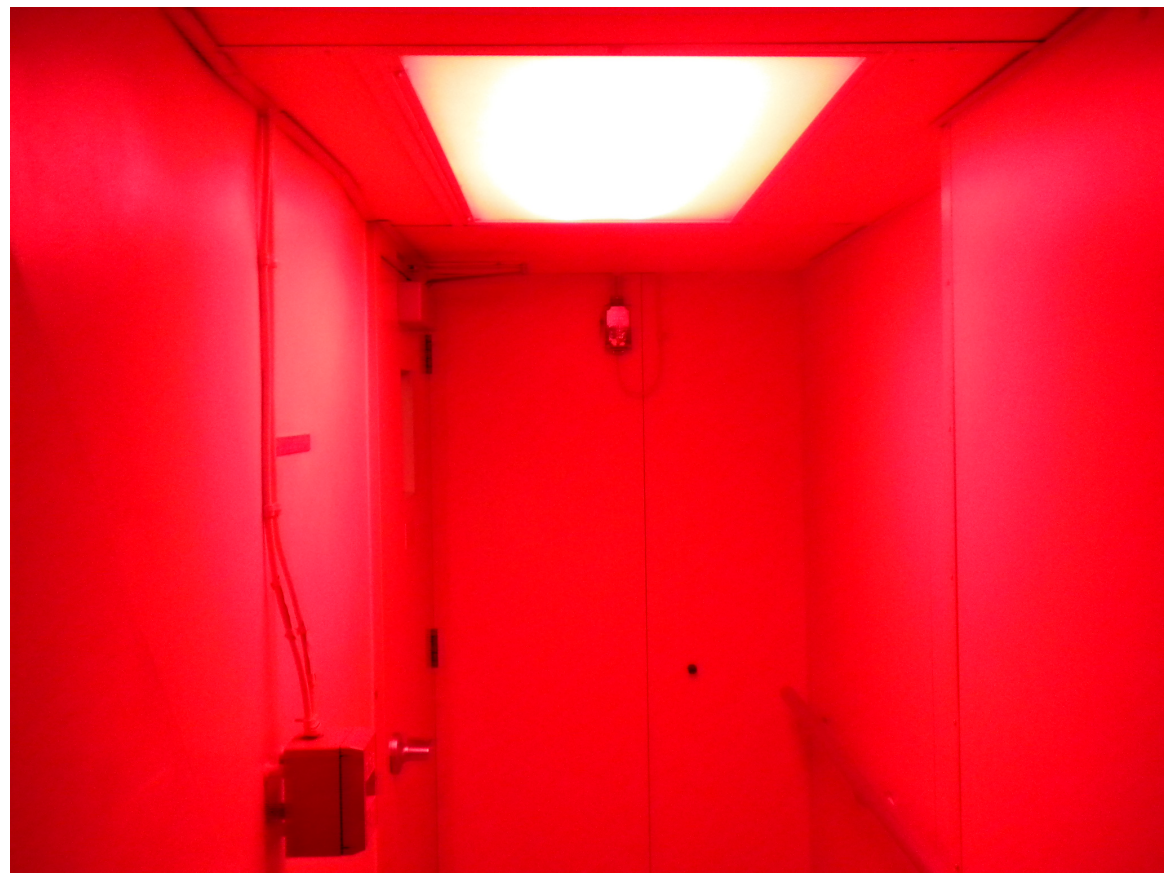

Figure 5.8: Picture taken during the Lumens expedition. 


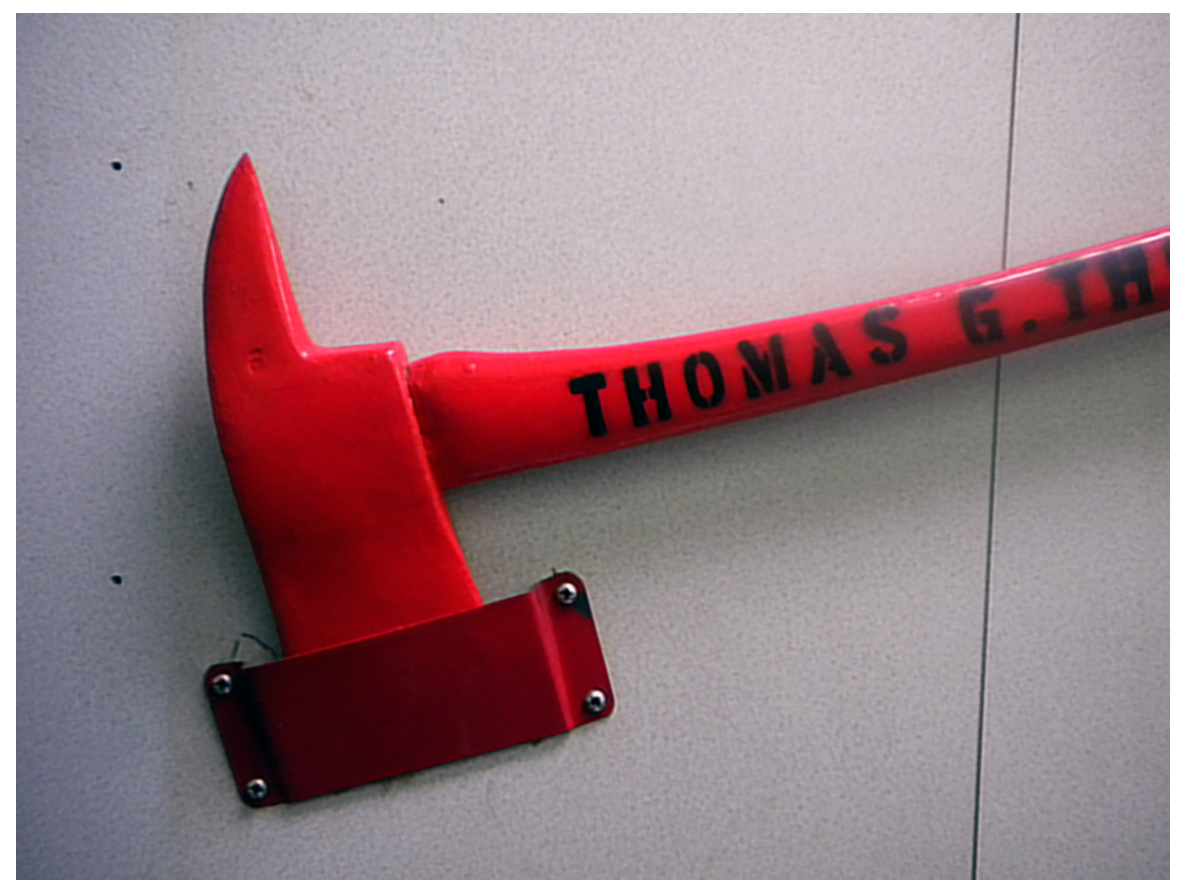

Figure 5.9: Picture taken during the Lumens expedition.

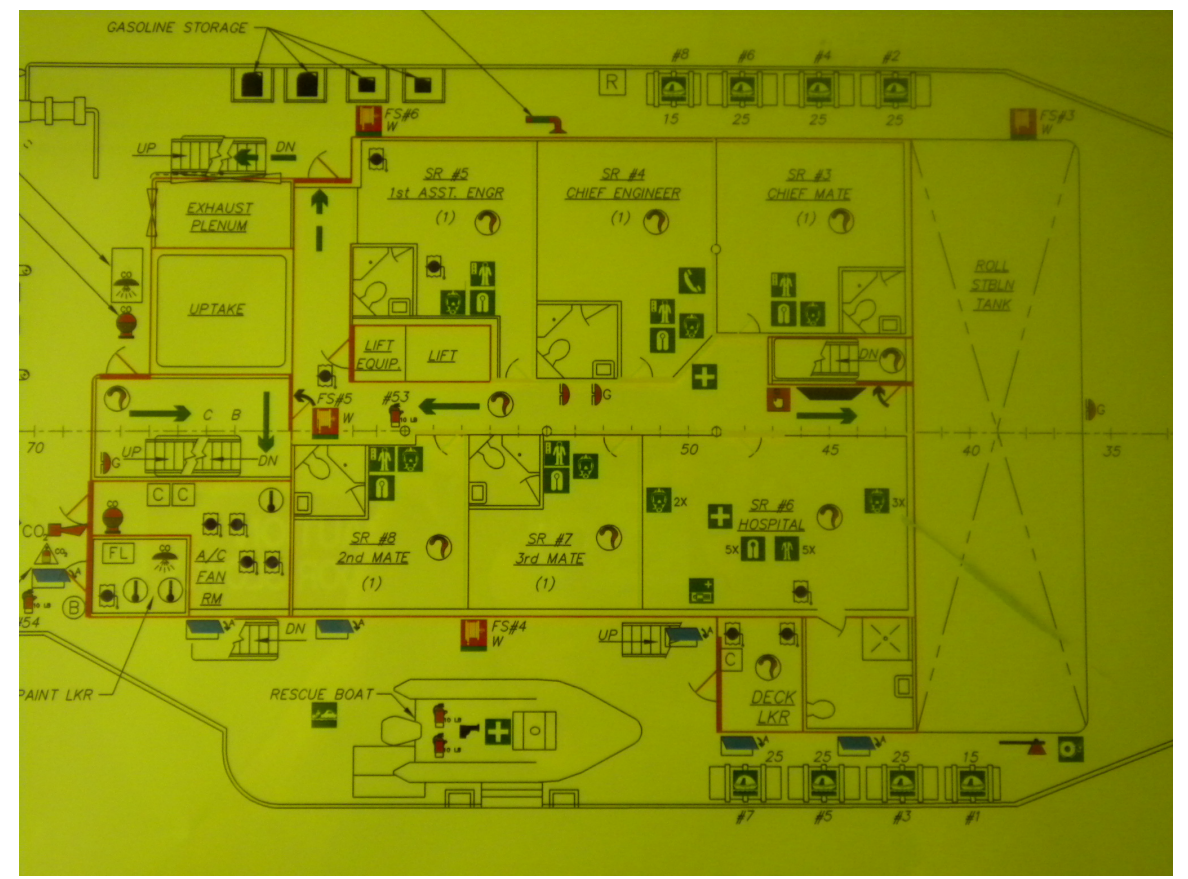

Figure 5.10: Picture taken during the Lumens expedition. 


\subsection{Initial Proposals}

\subsubsection{Proposal One: Laboratory Performance over the Sea}

The first idea for the implementation of the concept was setting up a kind of replication of a communication booth on the deck of an oceanographic ship. Adapted inside a cargo container, the space would be the hub for creating and delivering content to the external world. It would also be filled with different kind of objects that could produce sound such as sticks, tubes, hammers, and metallic plates, among others. In the form of audio improvisations, the artist would work as a translator of impressions between the human activity at the ship and the audience at land.

Inspired by Matthew Barney's Cremaster 3 (http://www.cremaster.net/), the idea of the proposal was to create a narrative of images with a high level of abstraction; a journey of experiences molded by the human body but at the same time with constant references to the sea. The performances would not be predefined, they would emerge from the actions and events happening on the ship as a set of unplanned improvisations.

Technologically extended by digital communication in real time, the artist (performer) would act as an observer of life aboard the ship and would respond with sound improvisations using both analog and digital sound sources. The improvisation would be broadcast over high-band Internet and people would be able to see the activity and products over the web.

Although some elements of this proposal were adopted in the final version, there were several obstacles to the realization of this version. There were concerns about the feasibility of the work, about lost Internet connections, and about the logistics of moving a loaded container. Conceptually the proposal did not make evident the importance of doing the process in real time, and the element of improvisation appeared to be more a sign of disorganization than a solid mechanism for sound production. In addition, there were concerns about all the empty periods during the day when no events or performances would be happening. The suggestion of the committee was to shape the experience into a more succinct work with fewer uncontrolled variables. 


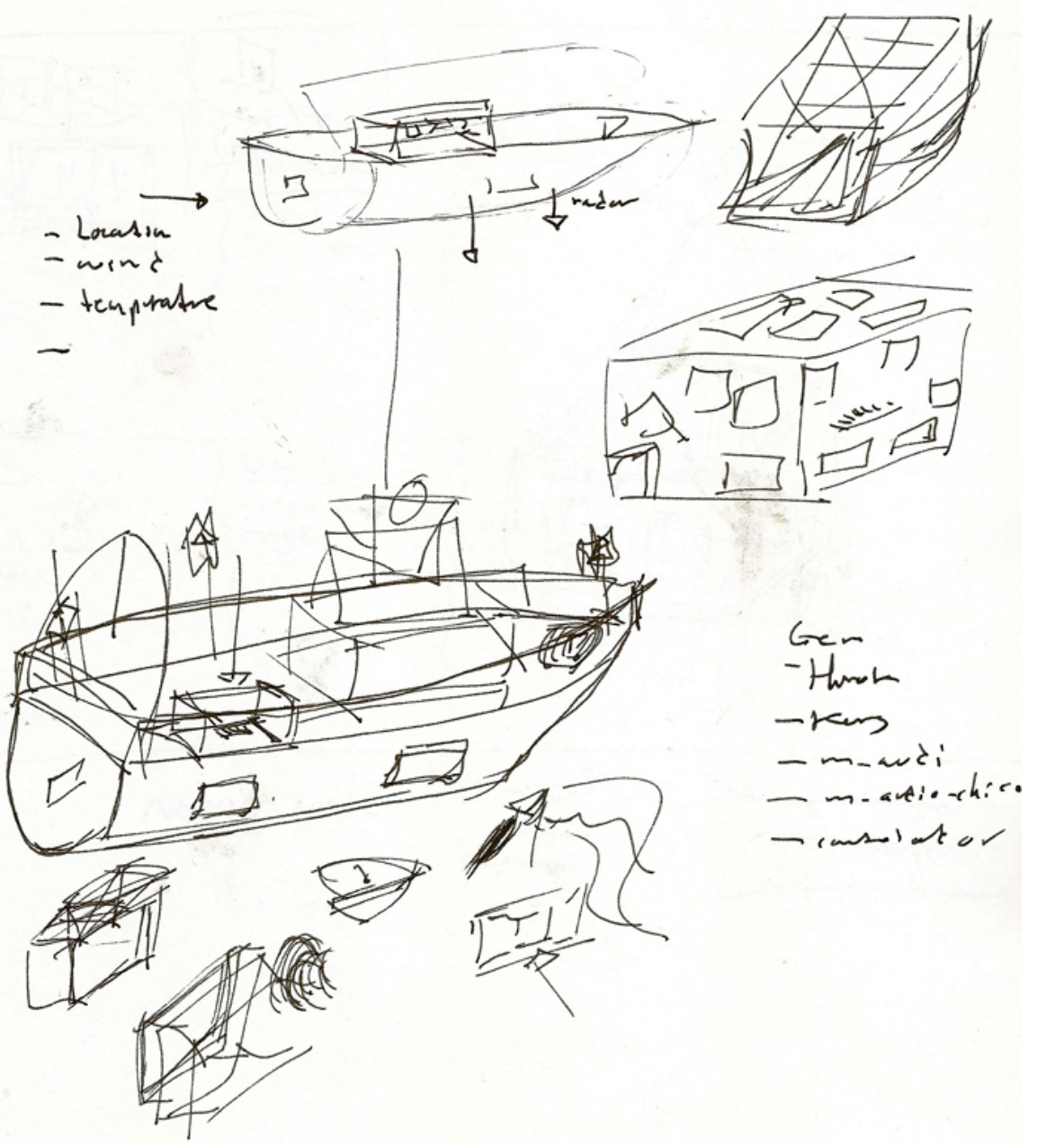

Figure 5.11: Sketch of proposal one. Laboratory performance over the sea. 


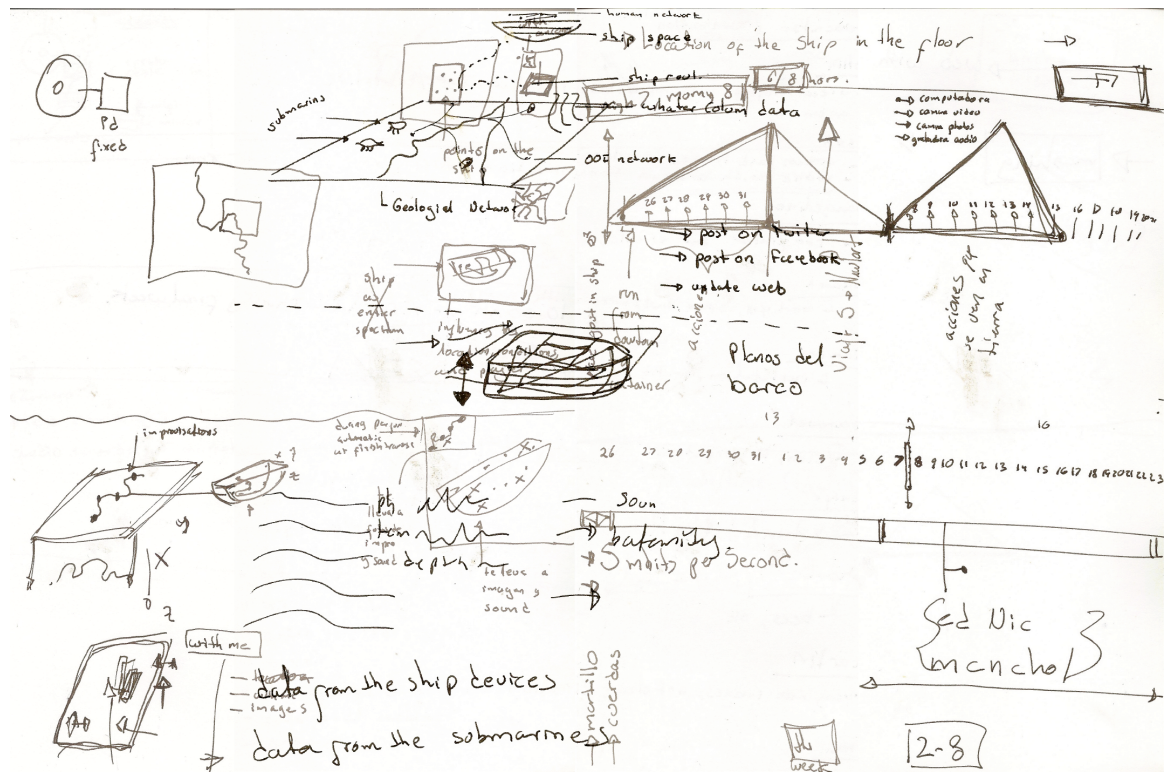

Figure 5.12: Diagrams of proposal two: multichannel telematic.

\subsubsection{Proposal Two: Multichannel Telematic}

Adapting the initial proposal into another format was a complicated process, mainly because some of the ideas became fixed in the imagination of the artist. It was hard to abandon some of the choices that originally seemed good selections. For this reason the second proposal kept several of the core ideas of the first version.

This proposal translated the communication hub and the performance activities to land. For this iteration, the artist proposed to receive the scientific data produced by the oceanographic ship and use it to modulate parameters of daily actions that should happen at the cargo container. The cargo container would be placed in an open field and would be used as data receiver hub, receiving data from the ship over an Internet connection. At one point the artist proposed to actually live inside the cargo container for a week, which would promote a physiological mood. A daily improvisation would be offered at sunset, using the container as a stage. It was suggested that this idea bore a resemblance to the work Two Undiscovered Amerindians by Coco Fusco and Guillermo Gómez-Peña (http://en.wikipedia.org/wiki/Coco_Fusco).

On the technical side, this version proposed the use of microcomputers, specifically 


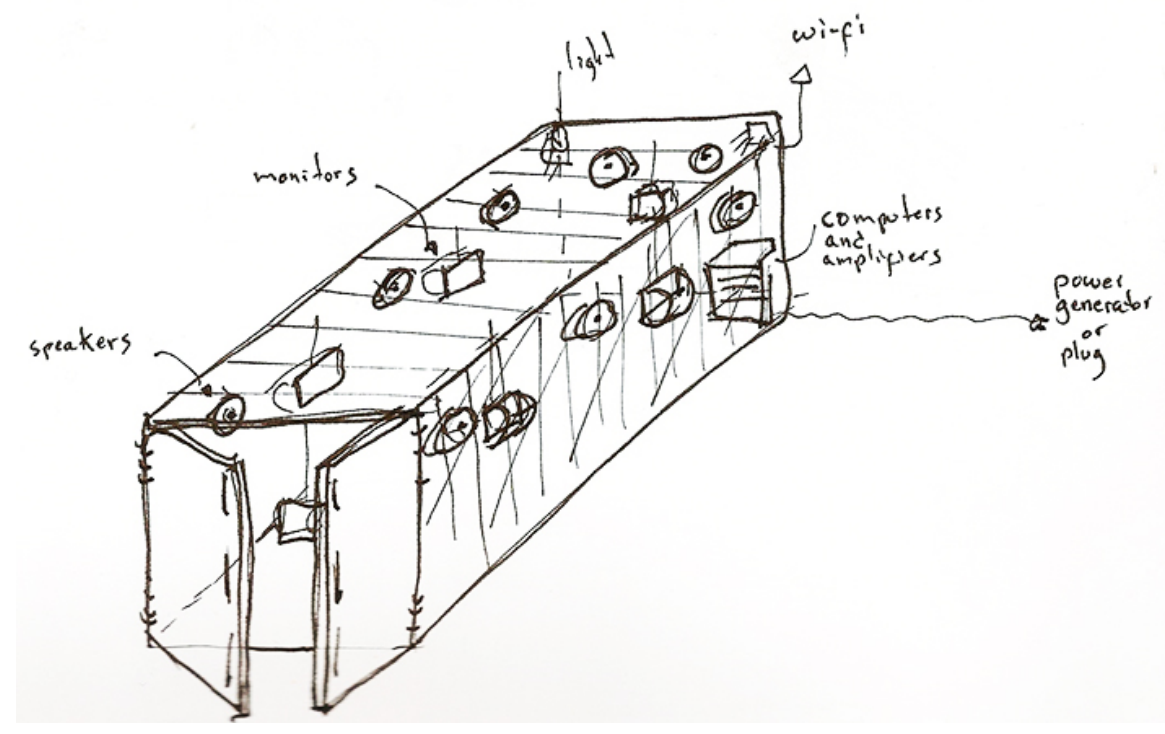

Figure 5.13: Sketch of proposal two: multichannel telematic.

the Mini2440 (http://www.friendlyarm.net/) as a economical solution for data processing, knowing that the majority of analysis and transformation could be performed on Amazon Cloud (see Referencesapp:cloudSynthesis). These microcomputers would also be a kind of embedded system inside the container, avoiding the use of obvious digital paraphernalia inside the container.

This version was also discussed with the university faculty and while feasibility was not a problem anymore, the ideas and artistic logic were still not clear. There was a lack of precision in the concepts. Too many ideas, too many media, and too many undefined situations made the proposal unclear. The main goal was diluted in the chaos of improvisation and the notion of liberty was misconceived.

At this point, the project was placed on hold for some months in order to decant some of the ideas and filter the elements that were not required and which were diluting the core idea. After this hiatus, with the influence of the works created during the residency at the Tokyo Wonder Site (http://www.tokyo-ws.org) and in Mexico City for the Transitio Festival (http://transitiomx.net), the final version was conceived and executed. 


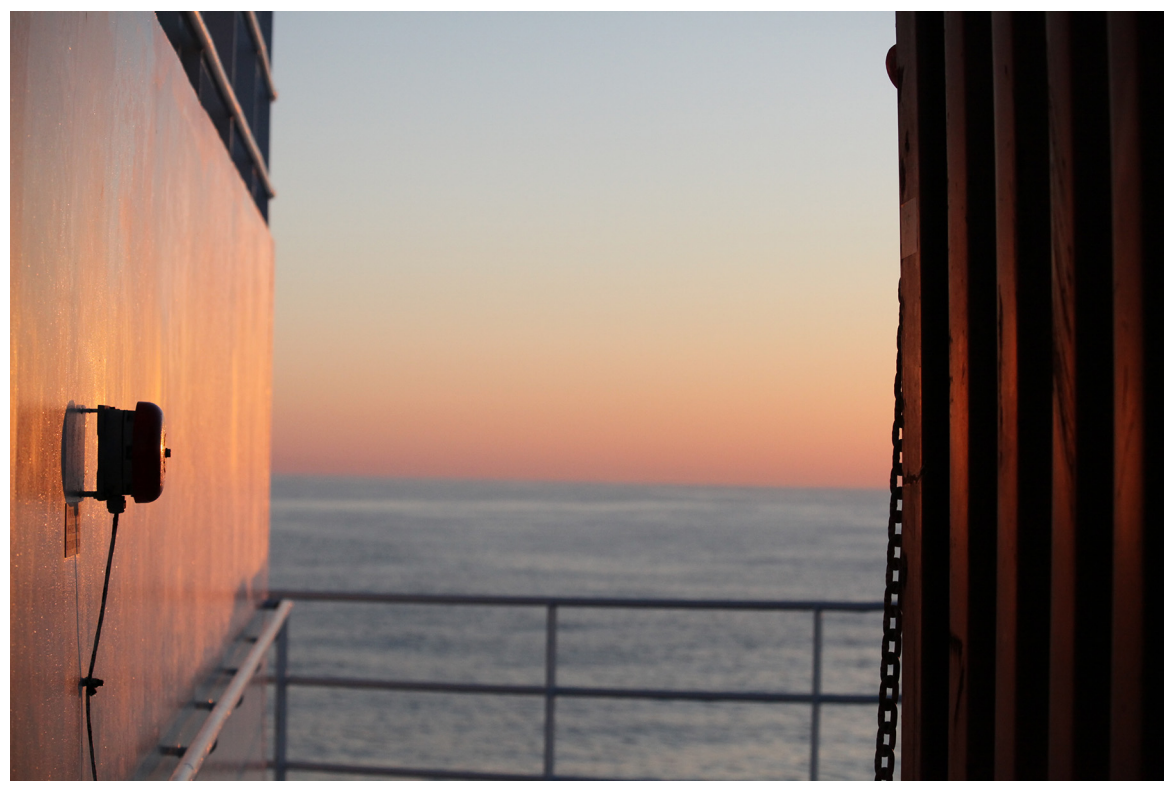

Figure 5.14: Container at ship. This image represents boundaries of space and location.

\subsection{Axial's Concerns}

After these initial stages of experimentation, trial and error, and conceptual evolution, the piece reached its final manifestation during the fall of 2011. The performative experience was sidelined and the aggressive sonic gesture emerged naturally as a sensorial translation of the sea force, the geological power, and the surrounding magnificence of the oceans. At the same time, the work acquired a simplicity of exposition in order to keep a natural dialog between the work and its natural surroundings.

As a complex work, the piece has several layers of interpretation, several relationships between the internal constitutive elements, several lectures according to the aesthetic perspective of the viewer, and many intersections between the inner logic of the piece and the surrounding reality where the piece exists.

As mentioned before, and as can be seen from the explorations and sketches that led to this final version, the work explores solutions, offers artistic responses, and generates artistic knowledge with the following concerns in mind:

Sound exploration This work uses sound as a medium to represent and manifest 
hidden elements and metaphors of visual and hidden reality. In this way, recording the hydrothermal vents and moving the robotic arm is part of this never-satisfied curiosity of sound art.

Dimension, proportion, and boundary The piece explores the notions of dimension, proportion, and boundary in order to represent the perceptual relationship between humankind and the rest of the world, specifically the ocean.

Space and void The piece explores the hidden contents of the void and matter that exist behind the surface (again, specifically the sea). Along these lines, the piece represents a gesture of curiosity and discovery.

Minimal gestures The piece searches for minimal and reductionist gestures in the compositional structures, rhythmic patterns, shapes, and display format as a mechanism to show the simplicity and long-term evolution observed in many natural processes.

Art, science, and technology This piece explores the intersection of art, science, and technology as a field to search and unearth the expressive relationships between the human and the environment.

\subsection{Undersea Soundscape}

\subsubsection{Recording Preparation}

After the first expedition, having collected a set of images and sounds, the oceanographic team introduced the author to Dr. Timothy Crone. Dr. Crone had recorded acoustic signals at black smoker vents as a way to study flow through sulfide structures, proposing that "changes in broadband components could be indicative of discharge rate variations related to the mechanics of tidal loading".[8]

Although Crone's concern was not musical or artistic, he had recorded and analyzed acoustic signals as a way to measure tidal patterns and also as a way to suggest biological implications. In order to realize these recordings, Dr. Crone and his team had to design and fabricate an acoustic recording system capable of producing long-term recordings 


\begin{tabular}{lll}
\hline & 1st Generation $(2004)$ & 2nd Generation (2005) \\
\hline Microcontroller & Persistor CF2 & Persistor CF2 \\
Hydrophone Element & Bentos AQ-2000 & Bentos AQ-2000 \\
Sample Rate & $1000 \mathrm{~Hz}$ & $1920 \mathrm{~Hz}$ \\
Number of Channels & 3 & 2 \\
A/D Converter & 12 -bit $(\mathrm{R} 212)$ & 16 -bit $(\mathrm{OES} \mathrm{AD24})$ \\
Anti-Aliasing & 3 -pole Bessel $(-3 \mathrm{~dB}$ at $500 \mathrm{~Hz})$ & 3 -pole Butterworth $(-3$ \\
& & $\mathrm{dB}$ at $500 \mathrm{~Hz})$ \\
Programmable Gain & No & Yes \\
Overall Gain & $-161 \mathrm{~dB}$ re $1 \mathrm{~V} / \mu \mathrm{Pa}$ & $-181-\quad-145 \mathrm{~dB}$ re 1 \\
& & $\mathrm{~V} / \mu \mathrm{Pa}$ \\
Dynamic Range & $\approx 72 \mathrm{~dB}$ re $1 \mu \mathrm{Pa}$ & $\approx 127 \mathrm{~dB}$ re $1 \mu \mathrm{Pa}$ \\
Storage Medium & 4 GB Compact Flash & $4 \mathrm{~GB}$ Compact Flash \\
Recording Capacity & $\approx 10$ days & $\approx 6$ days \\
\hline
\end{tabular}

Table 5.1: Recording system specifications. Table extracted from "The Sound Generated by Mid-Ocean Ridge Black Smoker Hydrothermal Vents". [8]

and able to record frequencies below $\tilde{1} 00 \mathrm{~Hz}$ with good quality. Between 2004 and 2005 Crone's team produced two versions of the recorder and captured several vent recordings. Figure 5.1 shows the different specifications of the two recorders.

After hearing about Crone's research, the author became fascinated with the possibility of producing sound field recordings at the seafloor, specially at vents and surrounding areas. Skip Denny from the OOI RSN engineering team lent the author some hydrophones in order to create some initial recordings during a testing period on Lake Washington and Puget Sound. Some hydrophones worked better than others but all of them registered boat motors and engines, mostly due the proximity to the sound sources. The author started to get familiar with the devices, equipment, and acoustic properties of sound recording in water. Recording below water produces a different kind of sonic material, especially with respect to echoes, resonances, reverberations, and low 


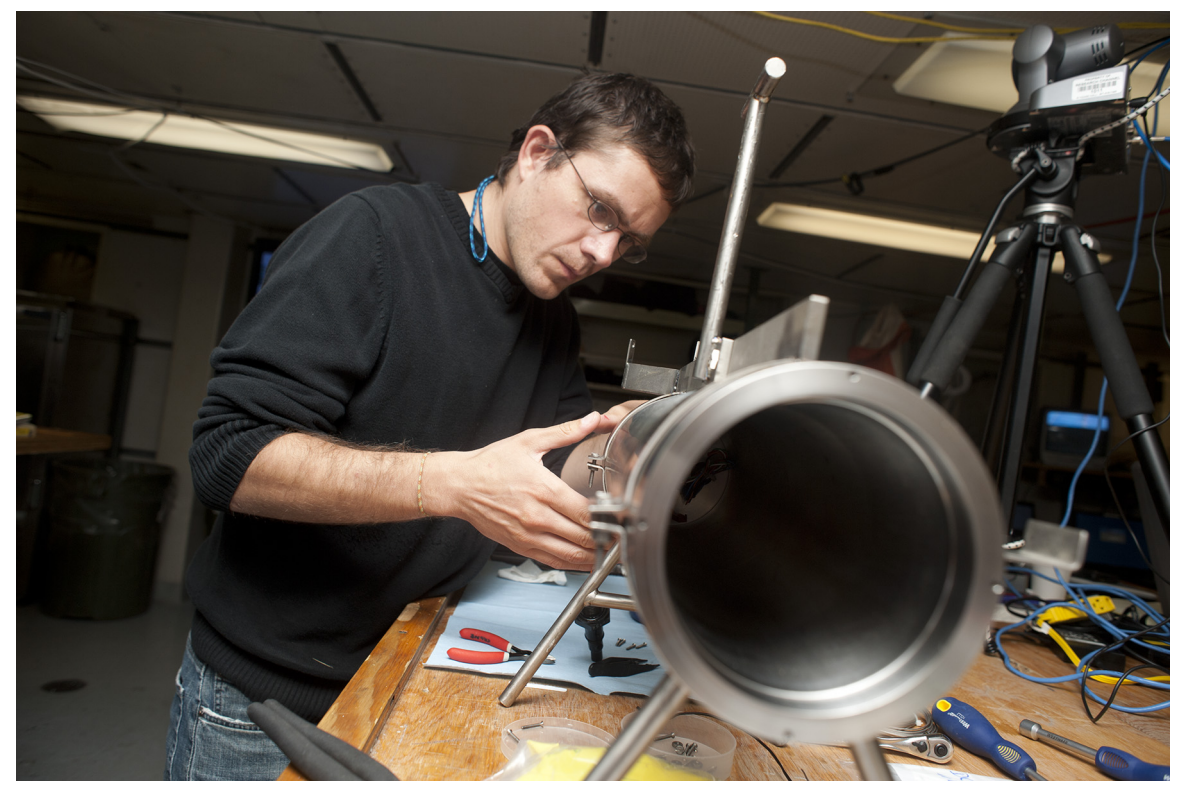

Figure 5.15: Preparation of recorders, placing two recorders inside the pressurized host.

frequencies.

Tim Crone and his equipment arrived in Seattle the day before the author departed on the Enlighten 2010 expedition. His work during the trip was testing and using video and computer vision analysis to calculate and infer flow speed at smokers and vents. Crone brought with him one of the audio recorders and the required equipment for creating sound recordings.

During the first couple of days aboard the ship, Crone explained to the author the procedures and techniques for setting up the recording including the deployment, placement in the ROV, recovery, and digital data treatment with Matlab. In addition to assisting Crone with his video experiments, the author and Crone set up the recorder known as VentPhone 2.0, which can record up to 48 interrupted hours of audio. However, it has a sampling rate of $1920 \mathrm{~Hz}$. While this sampling rate was good for the original purposes of the recorder, it was not ideal for signals in the audible range. Therefore, a second commercial audio recorder was employed in conjunction with the VentPhone.

The Zoom H2 is a portable commercial audio recorder. It records to an external memory card and can record compressed MP3 files of considerable size, depending on 

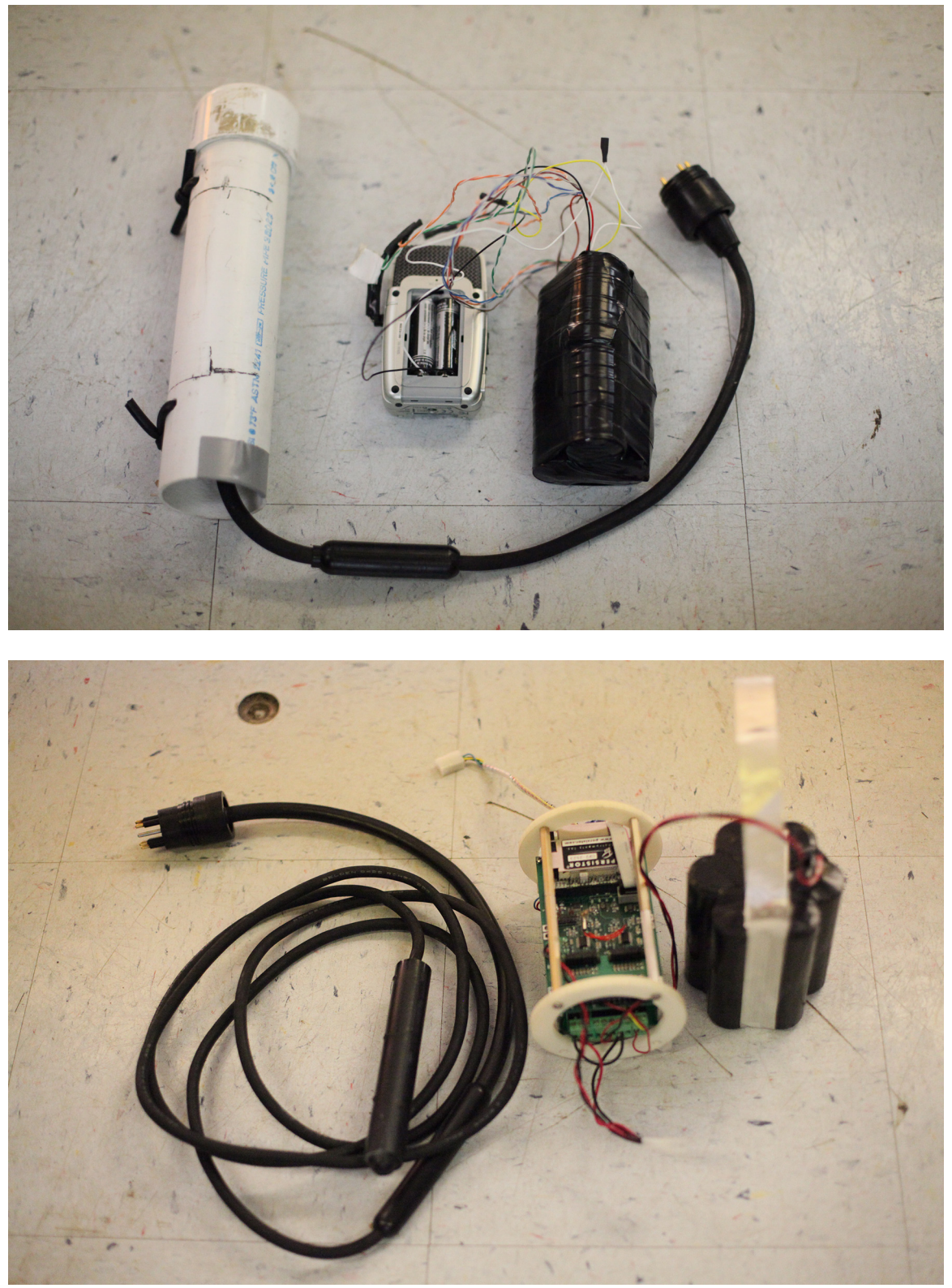

Figure 5.16: VentPhone and H2 recorders with battery packs and hydrophones. 


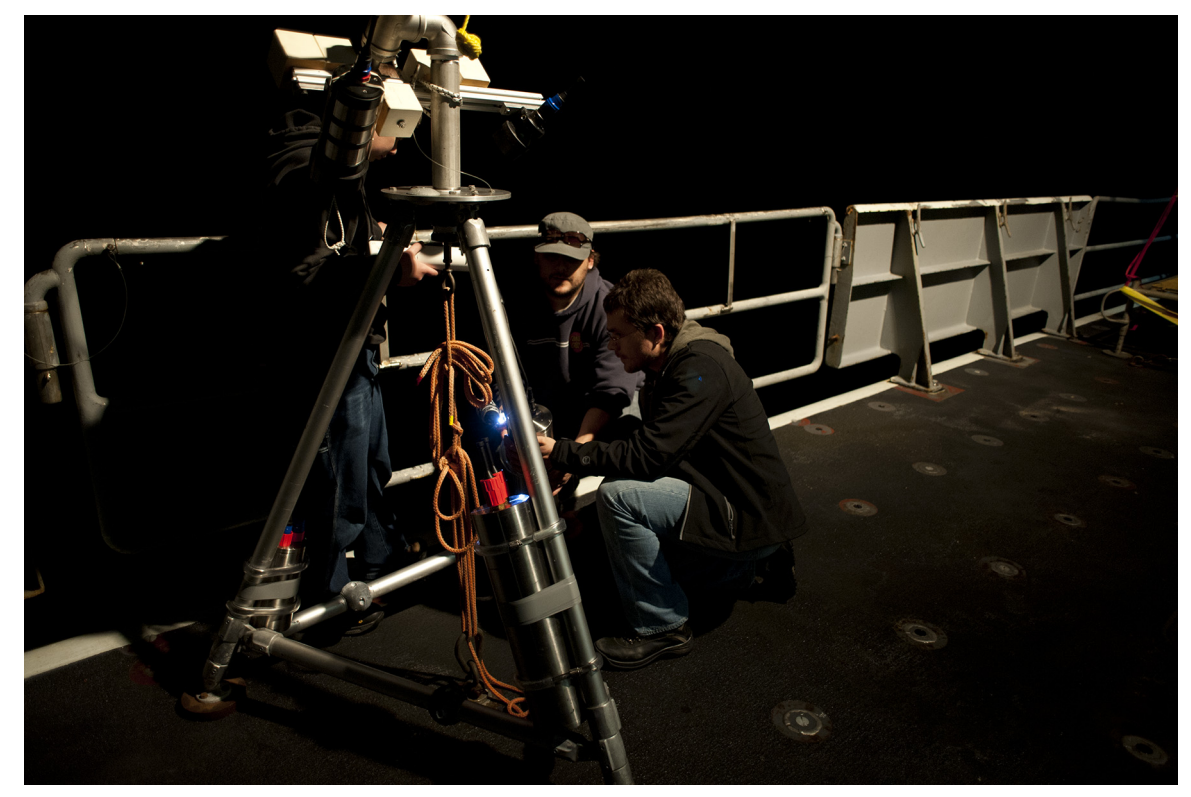

Figure 5.17: Attaching recorder and video camera to the tripod.

the size of the memory card. Since the recorder is not designed for long recordings, the batteries do not last for the required number of hours. Therefore we designed a set of external batteries for the 48 hours of the experiment. With this solution, both recorders were placed inside the pressure housing and the pressure housing was attached to the tripod.

On August 17, when the Thomson vessel was on top of the Medusa vent, the tripod was launched by free fall, with the proper balance of weights and buoys in order to slow the fall. Once the tripod was on the seafloor, the Jason Remote Operated Vehicle (ROV) moved it to a precise location in front of the vent. Jason removed the cap of the video camera and placed the hydrophone near the pipe of the vent.

All of the Jason's maneuvers are remotely controlled from the deck of the ship by the ROV's drivers. They use a set of cameras and sonar for real time visual assistance. The author followed the entire placement operation on screens inside the control room. When the author saw the arm of the ROV moving the hydrophone to place it at the vent, the author imagined an artistic gesture. Reaching that sound recording moment required a very non-trivial amount of planning, logistics, infrastructure, and support, 


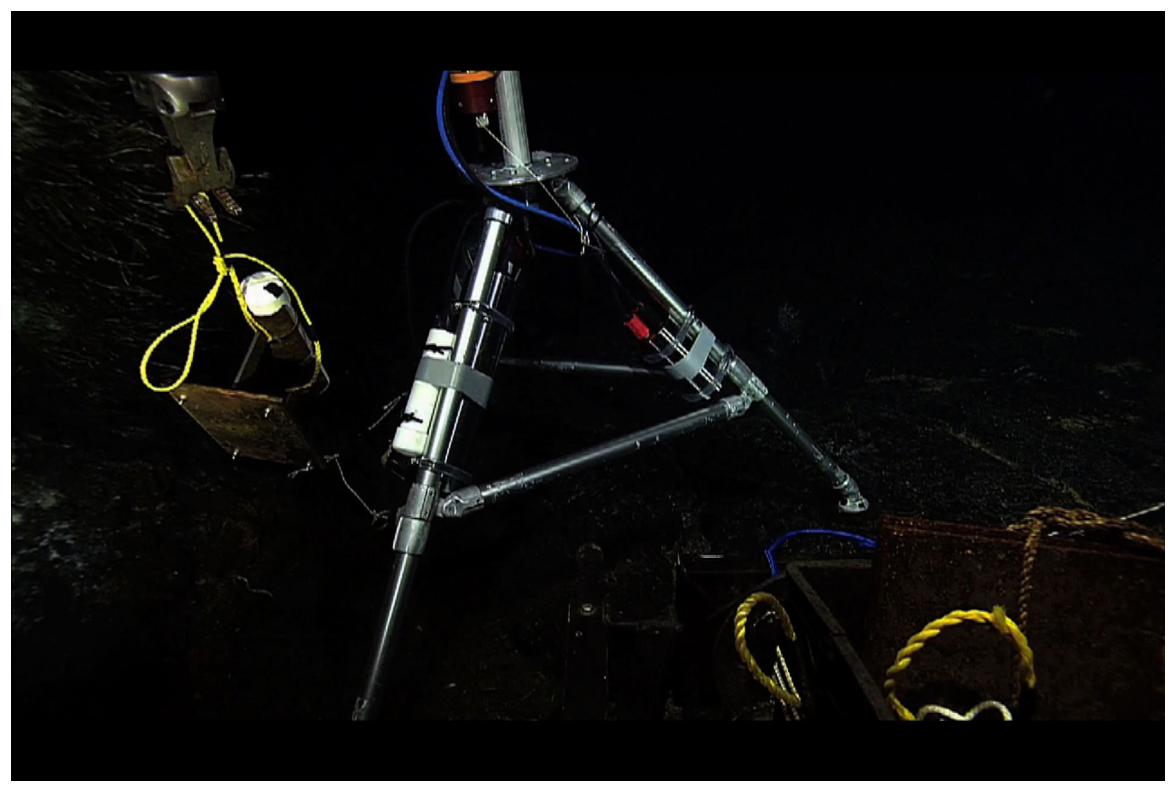

Figure 5.18: Jason arm locating hydrophone at hydrothermal vent.

all necessary to place a microphone in that remote location of the earth. Such action had the potential to become a statement and artistic gesture. The image of holding the microphone at the seafloor was so profound -beautiful, it could be said- that the author asked the scientist if it would be possible to have some time for guiding the ROV driver at the moment of the recovery, which was planned three days after the deployment.

\subsubsection{The Recording Gesture}

On August 20th, during the J2-518 dive of Jason, before the recovery of the tripod, there was a time window for the sonic experiment. Right after Dr. Crone did some camera movements with Jason's arm, he yielded the control for this work. While all cameras were recording from different angles, and right in front of the vent, the author ask the driver to grab the microphone and slowly reach the tip of the vent where the hot water flow emerged. Then the author asked the driver to move the micrpphone slowly back and forth, and in slow circles around the vent, mimicking the kind of gestures a sound artist employs when making field recordings.

The author's intention was to make evident as much as possible the performative 


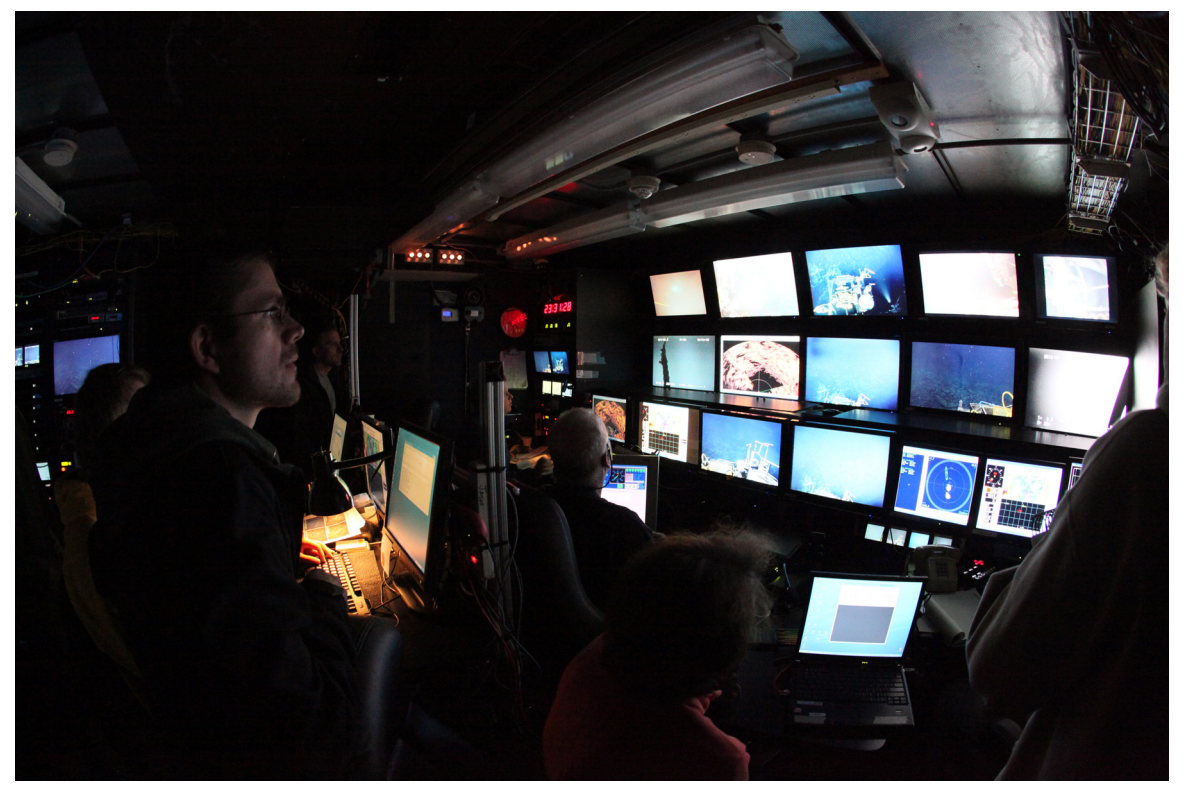

Figure 5.19: Guidance of Jason from the control room.

properties of the recording event and also to generate a narrative register of the situation. As mentioned earlier, recording sound on the seafloor is not trivial. Placing a recorder at 1,543 meters below the surface to satisfy a purely sonic curiosity is a challenge, a collaborative effort, and represents a communion between sound, art, and science. The action, which later was edited as a short video, represents the never-satisfied sonic curiosity of sound artists who transform and will transform all kind of matters, events, and actions into artistic expressions where sound plays a central role. The two-minute video became a visual element that works both as a documentary about the work as well as a manifest about sound art.

Once the action was done and the performance over, the tripod was recovered by attaching buoys that brought the equipment to the surface. The tripod was recovered with the vessel's crane and the materials were ready to be studied and analyzed at the ship. 


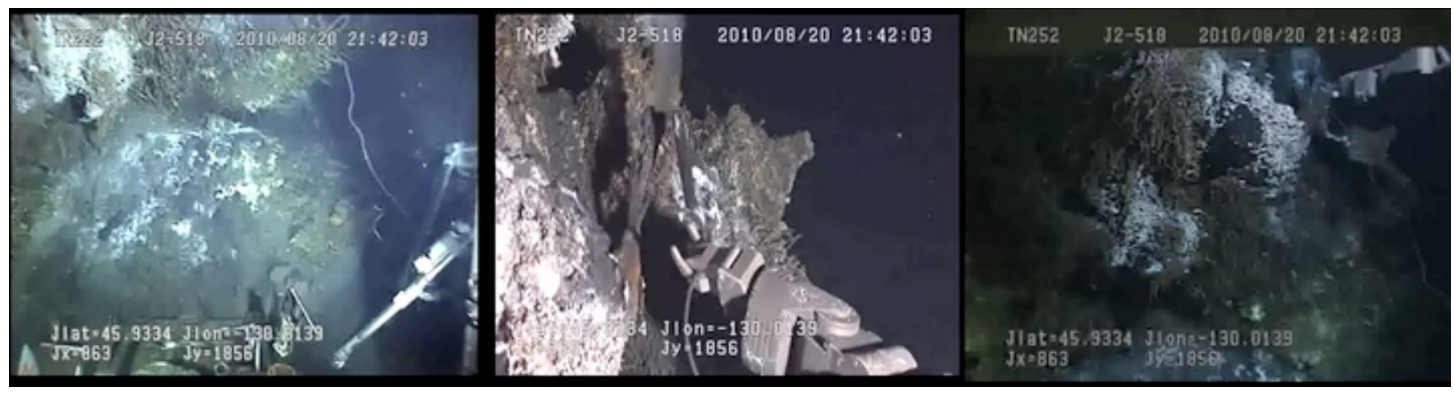

Figure 5.20: Jason's cameras recording the microphone recording.

\subsubsection{The Recorded Material}

The recordings from the seafloor were brought back to the ship for processing, editing, and manipulation. The total recording duration per recorder was 30 hours and 40 minutes. The signal shows clear data during the launching period when the tripod was deployed and also at the moment of placing the recorder on the precise site. After that, the signal became weak and sparse. After doing digital processing that included compression, equalization, normalization, and filtering the signal presented some of the vent sounds. The VentPhone recorder offered a clean signal without many harmonics due the sampling resolution, but the recorded material was extremely valuable due to the nature of the recording.

Unfortunately the $\mathrm{H} 2$ recorder presented a constant intermittent noise interference generated by the VentPhone's electronic circuits. Having such interference at the H2 recorder made it difficult to extract longer sound samples. There were, however, short windows of about three seconds that offered an excellent sonic panorama. The quality of the recorder offered a full spectrum sound, especially when the microphone was placed at the precise location by the ROV. This recording also shows the sounds of the ROV and the vessel when they were near the vent. The peculiar muted quality of sound traveling in water is appreciated on this recording.

The nature of the physical event produced steady low-frequency sounds. Musically speaking, the sounds do not offer a rich palette of direct transformation and manipulation since there are not short term variations. The sound can be described as a steady noise 


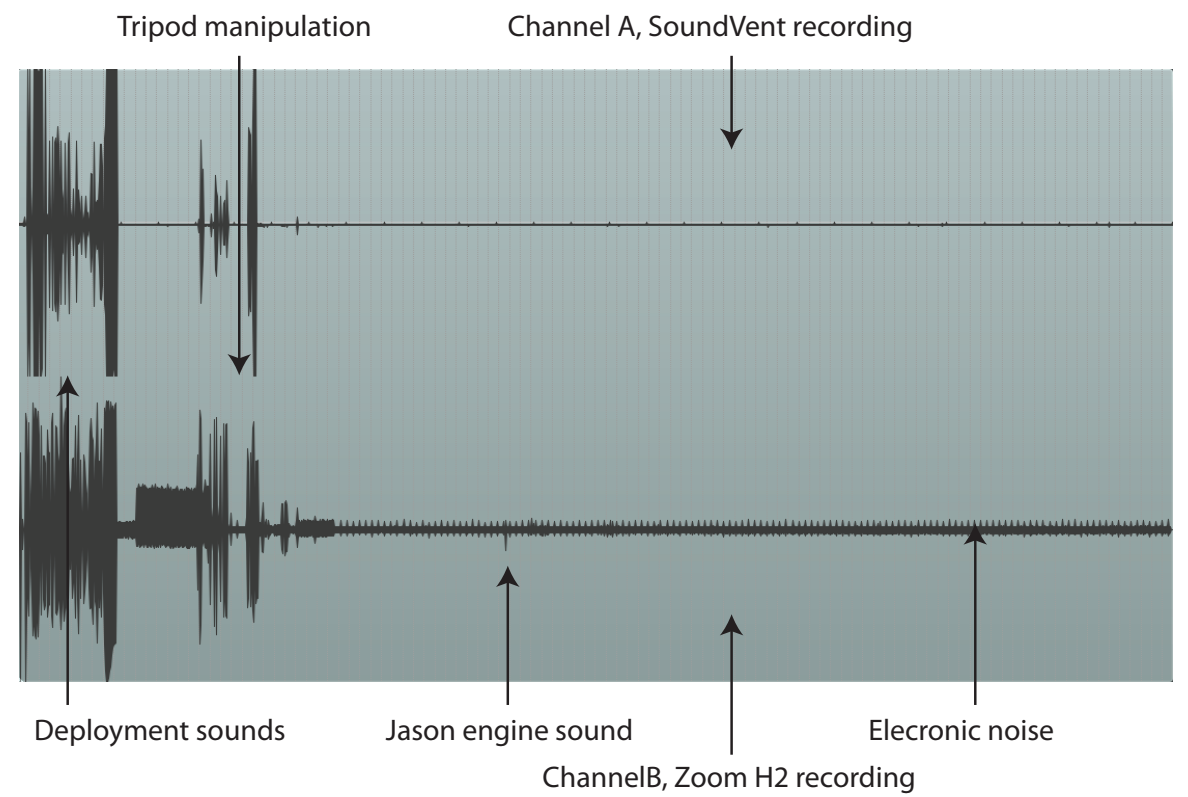

Figure 5.21: Waveform of first 30 hours of underwater recordings. Channel A shows the recordings of the VentPhone, channel B shows the recording of the Zoom H2 recorder.

with a resonator at the low frequency range. However the signal was interesting for modulating medium size structures and also as a sound source for some chains of digital processes.

\subsection{Sound Recordings on the Thompson}

Another activity performed during the Enlighten 2010 expedition was the creation of sound recordings at different locations aboard the vessel. The recordings complement the work and some of them were employed in the audio digital layer of the final work. The Thompson is $274 \mathrm{ft}$. in length, $52.5 \mathrm{ft}$. beam, and at full load has a draft of $19 \mathrm{ft}$. It is, therefore, a very big metallic resonator with several isolated rooms and heavy doors that separate the areas. The vessel is also an incredible sound generator, and a wide variety of sounds and textures can be recorded on it. The engine room, scientific labs, kitchen, pipes all around the floor and ceilings, deck, corridors, communication room, cranes, containers- these are some of the spots that generated a variety of sonic textures. 
Depending on the nature of the material and generated sound, different microphones were employed for the recordings, as described below ${ }^{2}$.

Stereo microphone Regular ambient sounds, walking around the corridors, and some deck observations were recorded using a regular stereo microphone.

Ambisonics microphone A couple of deck observations and many of the room registrations were recorded using an Ambisonics microphone. From this set of recordings, the recordings made inside the bow of the vessel deserve special attention. When in motion, waves repeatedly strike the bow, where a small storage room is located. The room is partially empty but metallic devices are hanging from the metallic walls. The sounds generated by each wave hitting the vessel are extraordinary in terms of rhythmic patterns and acoustic energy. The acoustic experience is complemented by the fast up and down movement of the vessel, which generates vertigo and whirl. Recording the situation in the Ambisonics format with the best quality possible for future processing and manipulation was valuable and important for the project.

Contact microphone The vessel is equipped with an incredible array of devices: hydraulic, pneumatic, electric, and digital equipment is spread over the entire vessel, producing micro-sonic worlds that were recorded with contact microphones. Exploring the sonic properties and surprises of laboratory equipment was also part of the research and discovery process of building the piece.

Hydrophone Sea recordings were registered using hydrophones hanging from leeward and windward. These recordings register the water hitting the hull and also the operation of the two $3000 \mathrm{hp} \mathrm{Z-drives} \mathrm{that} \mathrm{move} \mathrm{the} \mathrm{vessel.}$

\subsection{Data at the Ship}

The Thompson vessel is equipped with devices for measuring a wide variety of geographic, chemical, physical, and geological information. See section Technology at the

\footnotetext{
${ }^{2} \mathrm{~A}$ video documentary about the recording process was kindly produced by the OOI team (see Referencesapp:materials)
} 


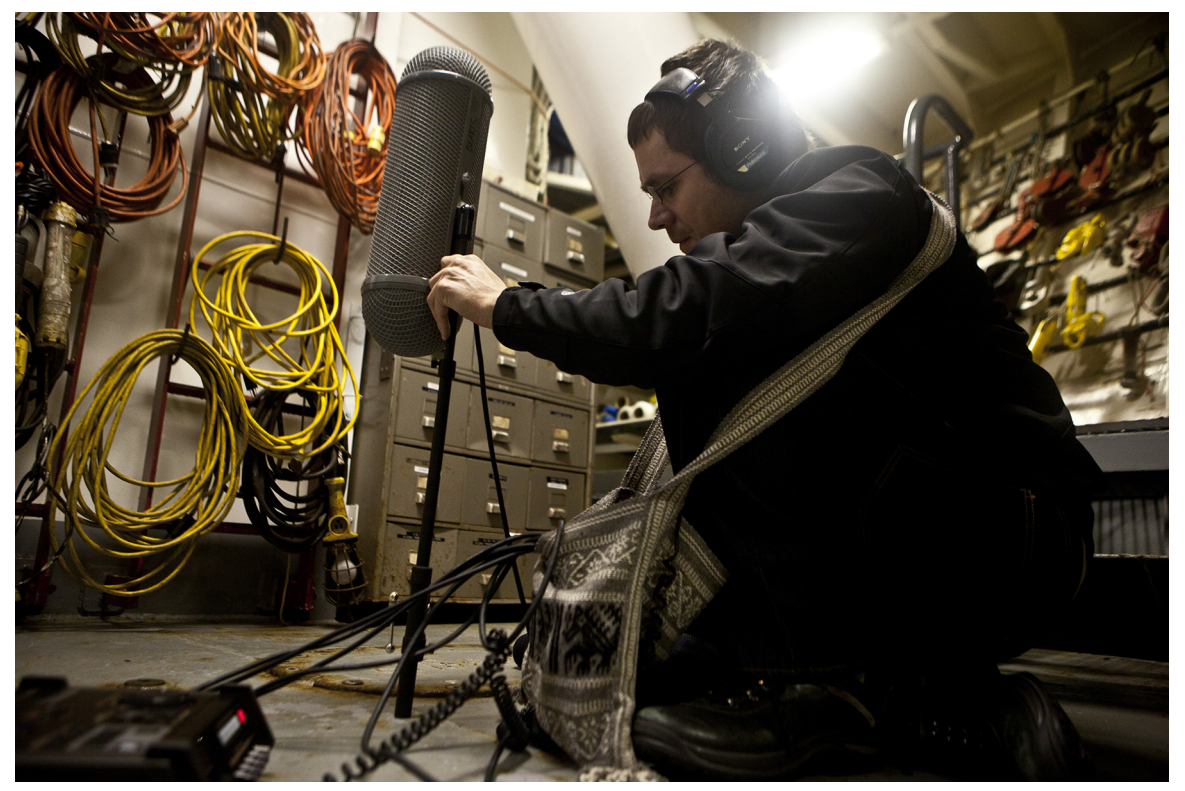

Figure 5.22: Sound recording in the bow of the Thompson.

OOI for the list of equipment and the uses of each device. The ranges, sampling rate, sampling resolution, and variability of the data is different depending on the registered physical event but all of the data can be adapted and normalized to appropriate ranges. It is important to point out that the data described in the rest of the chapter was used to modulate sonic parameters. The science team generated much more data and information, such as CTD values, but only some of them were used for the artistic project. Which materials and how they were interpreted as which sonic element is detailed in Composition and Deployment.

Most of the data consists of a single value that slowly varies over time. Slopes and spikes are rare on water profiles, where gradual changes are expected. On the other hand, bathymetric maps offers spikes and valleys. Sub-buttom profiles offer multilayer images of extraordinary sonic attraction that will be described later in this chapter.

\subsubsection{DAS}

Among the different data that the scientists produced during the expedition, we encounter a collection of information registered from equipment preinstalled on the vessel. 


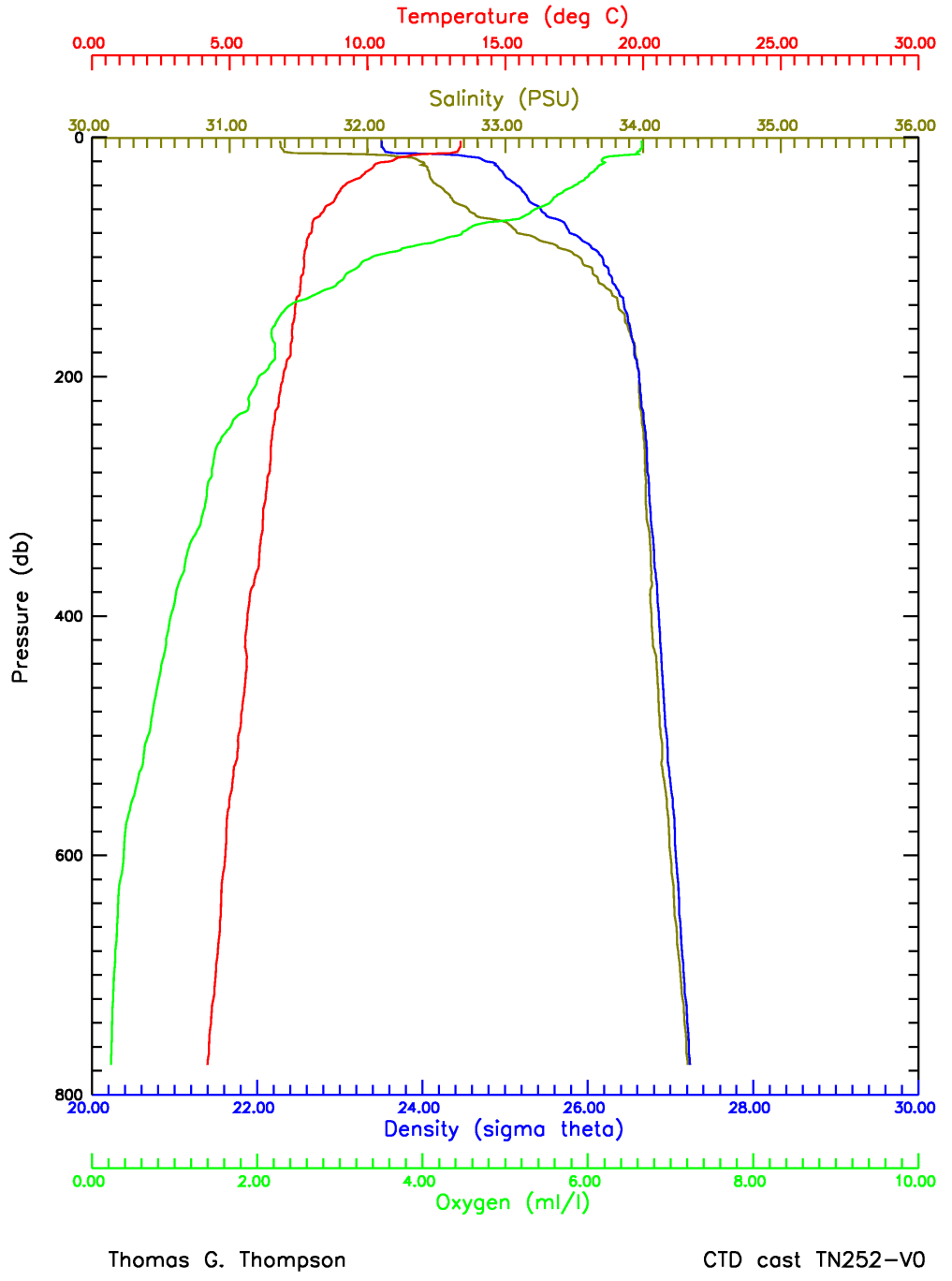

Figure 5.23: CTD plot showing temperature, salinity, density, and oxygen changes. 
This collection of data is known as DAS. These recordings are part of the regular register of all expeditions. Table DAS parameters shows all the parameters that are registered. These materials were exported as text files, manipulated with custom Groovy scripts, and read as SuperCollider buffers.

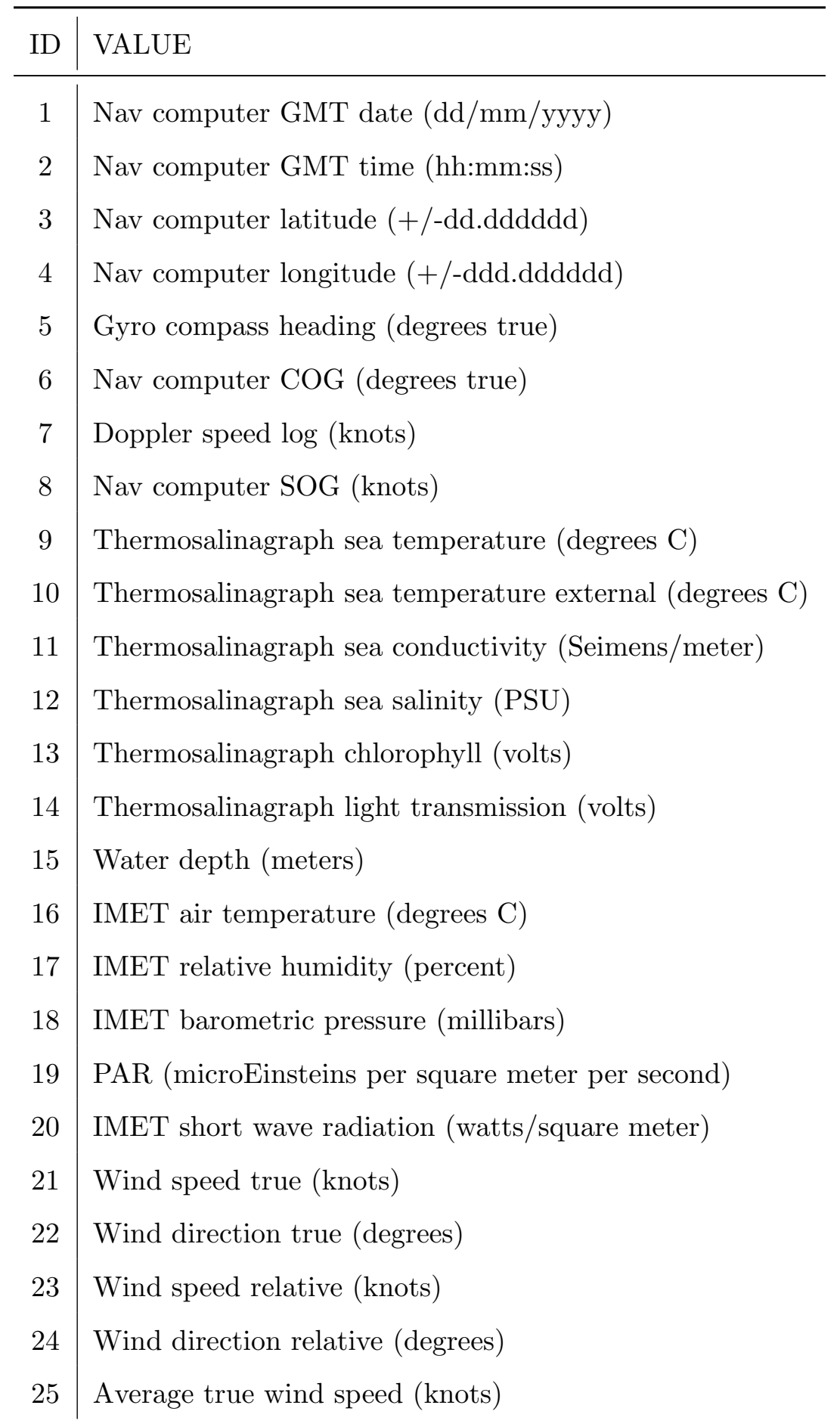



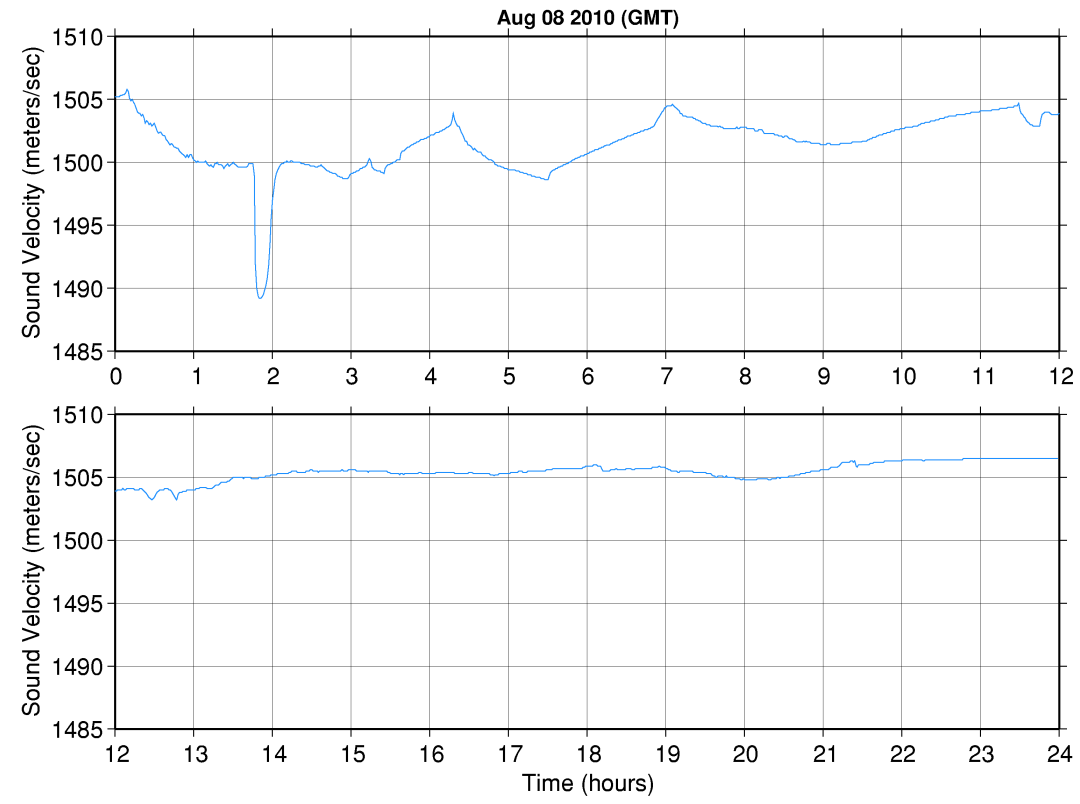

Figure 5.24: Plot of sound velocity changes over 24 hours.

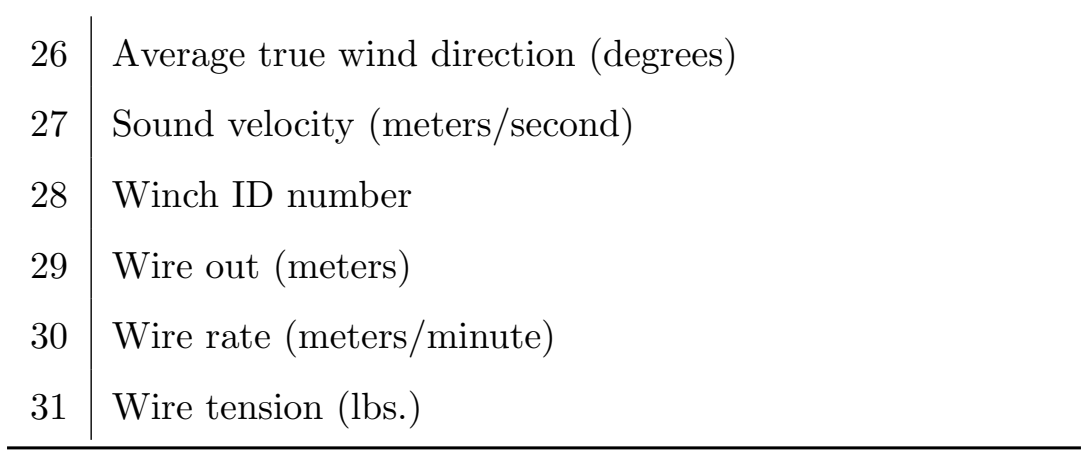

Table 5.2: Elements logged during expedition (DAS).

\subsubsection{Bathymetry}

As described in section Bathymetry of the Seafloor, bathymetry is the measuring of the deep sea floor. One of the principal objectives of the Enlighten expedition was the 


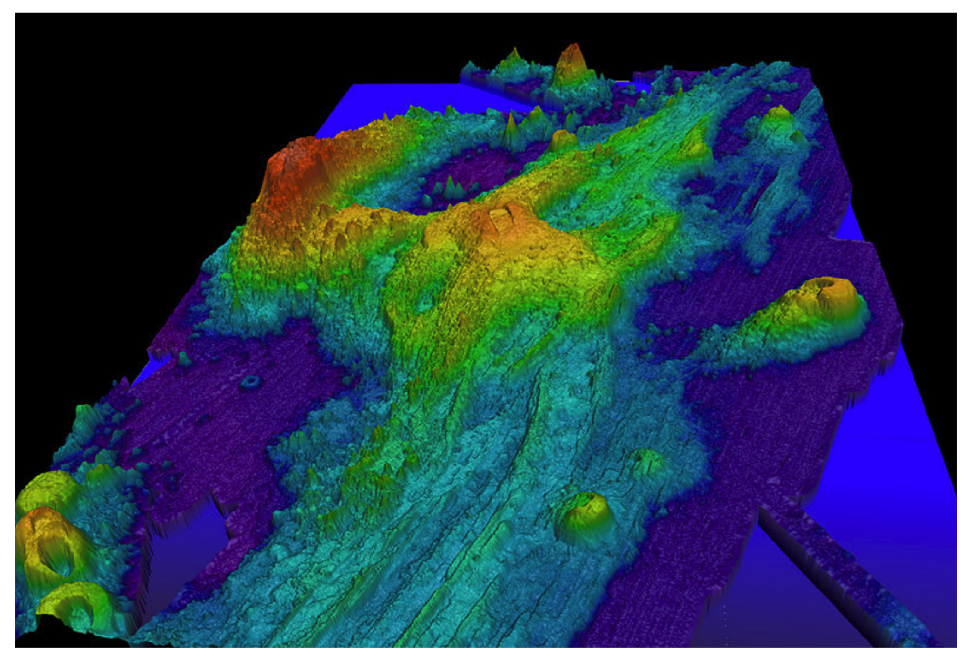

Figure 5.25: Bathymetry of Axial seamount.

generation of high-resolution bathymetric measurements of the region in order to define the best location for cables and nodes of the Ocean Observatory. For this reason, several devices and techniques were employed for measuring the elevation of the sea floor. The EM300 multibeam echosounder mounted on the hull of the Thompson generated maps with a 50-meter resolution. The RESON SeaBat multibeam sonar aboard the Sentry and the Jason created fine-scale resolution maps.

The different bathymetric data were exported as text files, and in the case of the DAS data, manipulated and transformed with custom Groovy scripts for feeding SuperCollider buffers which later modulated sonic parameters.

\subsubsection{Sub-bottom Profiles}

With the Knudsen Chirp 3260, sub-bottom profiles were obtained. The main interest of this data for our purposes is that this material provides a multilayer information. While bathymetry and DAS information offers a single value over point/time, subbottom profiles represent the different layers that composes the seafloor. Therefore, the Knudsen profiles can be interpreted as a sonic spectrogram. 


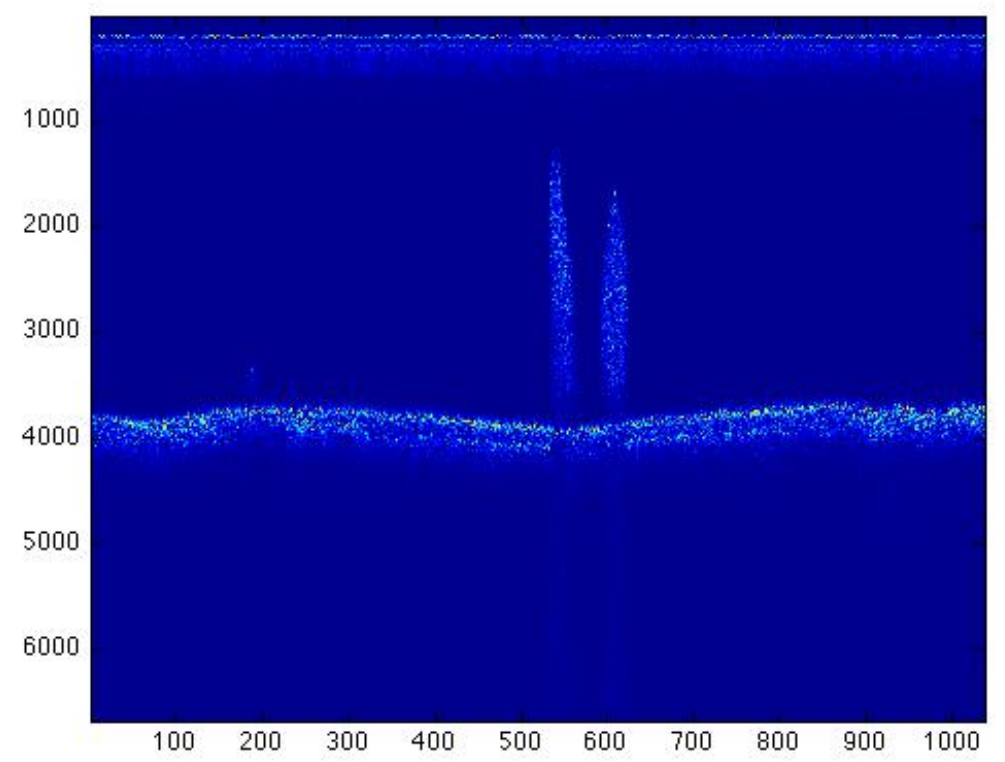

Figure 5.26: Sub-bottom profile showing two pipes.

\subsection{Cloud Synthesis}

Cloud Synthesis is the name the author coined to refer to the execution of offline sound synthesis and processing using the cloud infrastructure. In several courses and classes about computer music, professors of previous generations have recalled that when they started to compose computer music, they had to wait for days in order to listen to a finished rendering.

In contrast, computer power today seems fast enough for most sound processing activities. Real time multichannel synthesis with unnoticed latency is feasible at extremlye high sampling rates on standard portable computers, and rendering of complex processes if performed quite quickly. On the other hand, people working on video animation and video effects are still waiting a significant amount of time in order to see the final product. The use of distributed processing and farm rendering is therefore common in graphics production.

Has computer speed reached a level that satisfies most of the power requirements 
of digital sound? For regular situations current speed is enough but speed may still be a factor to consider for big multichannel works, 3D sound scenes, and remote locations with limited computer access.

The author started to create a distributed rendering infrastructure more out of curiosity than from the necessity of power or speed during Proposal One: Laboratory Performance over the Sea. During that period the author took a course in the Computer Science department that uses Android and Google Engine to leverage processing on portable devices. Right after the course, Amazon offered a set of workshops about their cloud system. Later, knowing that a significant amount of data would be produced by the oceanographic team, the author saw an opportunity to apply the knowledge in order to generate an original solution.

With those antecedents, the author worked on a digital infrastructure that takes advantages of Amazon Web Services (http://aws.amazon.com/), allows the rendering of sound scenes using a small coordinator instance as web service and as many SuperCollider servers as needed. The details about the entire system are explained in Cloud Synthesis.

\subsection{Conclusions}

As can be seen, the preparation, conceptual journey, and materialization of the project took a significant amount of time. Several different ideas and artistic logics were explored and developed in order to generate the work. All these ideas, sketches, testing, and preparations were of extreme importance in order to finally arrive at the finished piece. The final iteration of the idea that became the artwork is explained in the next chapter. 


\section{Chapter 6 COMPOSITION AND DEPLOYMENT}

“...But pardon, and gentles all,

The flat unraised spirits that have dared

On this unworthy scaffold to bring forth

So great an object: can this cockpit hold

The vasty fields of France? or may we cram

Within this wooden $O$ the very casques

That did affright the air at Agincourt?

...Piece out our imperfections with your thoughts;

Into a thousand parts divide one man, And make imaginary puissance;

Think when we talk of horses, that you see them Printing their proud hoofs i' the receiving earth; For 'tis your thoughts that now must deck our kings, Carry them here and there; jumping o'er times, Turning the accomplishment of many years Into an hour-glass: for the which supply, Admit me Chorus to this history; Who prologue-like your humble patience pray, Gently to hear, kindly to judge, our play." William Shakespeare

This chapter focuses on the composition of the work. Once the compositional idea became clear, the materials collected, the artistic context established, and the technology developed, it was the moment to materialize the idea into an observable work. This chapter presents the description of the sound installation that constitutes the actual artistic work, composed and elaborated with the ideas, concepts, logic, tools, and tech- 
niques described in the previous chapters. This section describes the properties, setup, installation procedures, and characteristics of the work in all its elements including physical properties, hardware, sound recordings, and sonic structure.

\subsection{Ideas About Composition and Creativity}

Explaining the compositional process is, for the author, without question the hardest step in explaining the entire process of Axial's development. Explaining a compositional process requires a difficult and complex self-analysis. Scientists, theorists, and philosophers have written serious theses about creativity, art creation, and composition processes, but personal introspection about how a piece is created is still complicated.

While it is hard to formalize and present a set of rules or set of instructions, there is a consensus that art composition is linked to imagination and free exploration. Evolution, transformation, and variation of previous works that society and critics point to as valuable works may be a good path for original creation. In this sense, the study and analysis of referential works is important and, while there are exemptions, most works derive from previous works.

While form and style are relatively clear concepts that remain stable for long periods of art history, in the 20th century such concepts became diluted by a set of complex justifications about liberty. Artists of the 1970s and 1960s broke paradigms and established other kinds of relationships between artworks, viewers, and tradition. Such revolution and evolution was reasonable and logical but we in the younger generation have inherited a complex panorama. While everything seems to be valid on the surface, at the bottom we still face the inevitable forces of perception limits, archetypes, and social memory.

It will take time to know if what we now know as New Media and Experimental Art will in the future have the artistic validation of other creative manifestations; in the meantime, we as artists will take advantage of the times we live in and explore the possibilities that offer working on multiple platforms, working with a variety of technologies, and creating artistic proposals that lie on the intersections of art and technology, art and science, art and sensorial experiences, and so on. 


\subsection{Compositional Process}

As mentioned in the chapter Preliminary Work the ideas, images, concepts, and elements of the project had to be materialized into a formal art piece that could poetically encompass the desired logic. Several formats were conceived and proposed, and some were discarded along the way by the author, while some were discussed with the faculty committee and revised after discussions and observations.

After months of planning, the piece was shaped and materialized into the final iteration. Specific materials were selected, specific techniques employed, and specific pieces of code written according to the sonic experience. In other words, decisions had to be made in order to achieve a finished piece. How was the compositional process? How was the piece shaped into its final manifestation? Let us review a set of time facts.

The idea to work with the sea as a final project was conceived in 2008 while living on Puget Sound. During that time, the author took the "Mechatronics Art" and "Sound Sequence" courses at DXARTS and some courses in the Computer Science department at the University of Washington. In 2009, the author took courses in the Oceanography department and also attended the Lumens expedition. In 2010 the author took the course "Sound Art" with Juan Pampin at DXARTS, detonating a set of sonic paradigms. The same year, the author participated in the Enlighten expedition and collected the required materials. A set of explorations were proposed and started but never solidified into a finish work. In 2011, the author produced the Plurifocal modules while doing an art residency in Tokyo, Japan. A couple of works from that period influenced Axial's proposal and lent a new approach to the project. During this period, the idea of working with a mechanical component gained force and two artworks using similar principles were created as preparation and testing for Axial.

In August of 2011, everything was set up and enough exploration had been produced around the main concept of the piece. None of the previous activities was consciously organized or planned specifically for the materialization of the piece as it was ultimately produced. Up until that point there was a set of opportunities, heuristic explorations, curiosity, and informal research gradually evolving. Everything remained as a combination of sonic desires, as the production of a variety of artworks not necessarily related to 


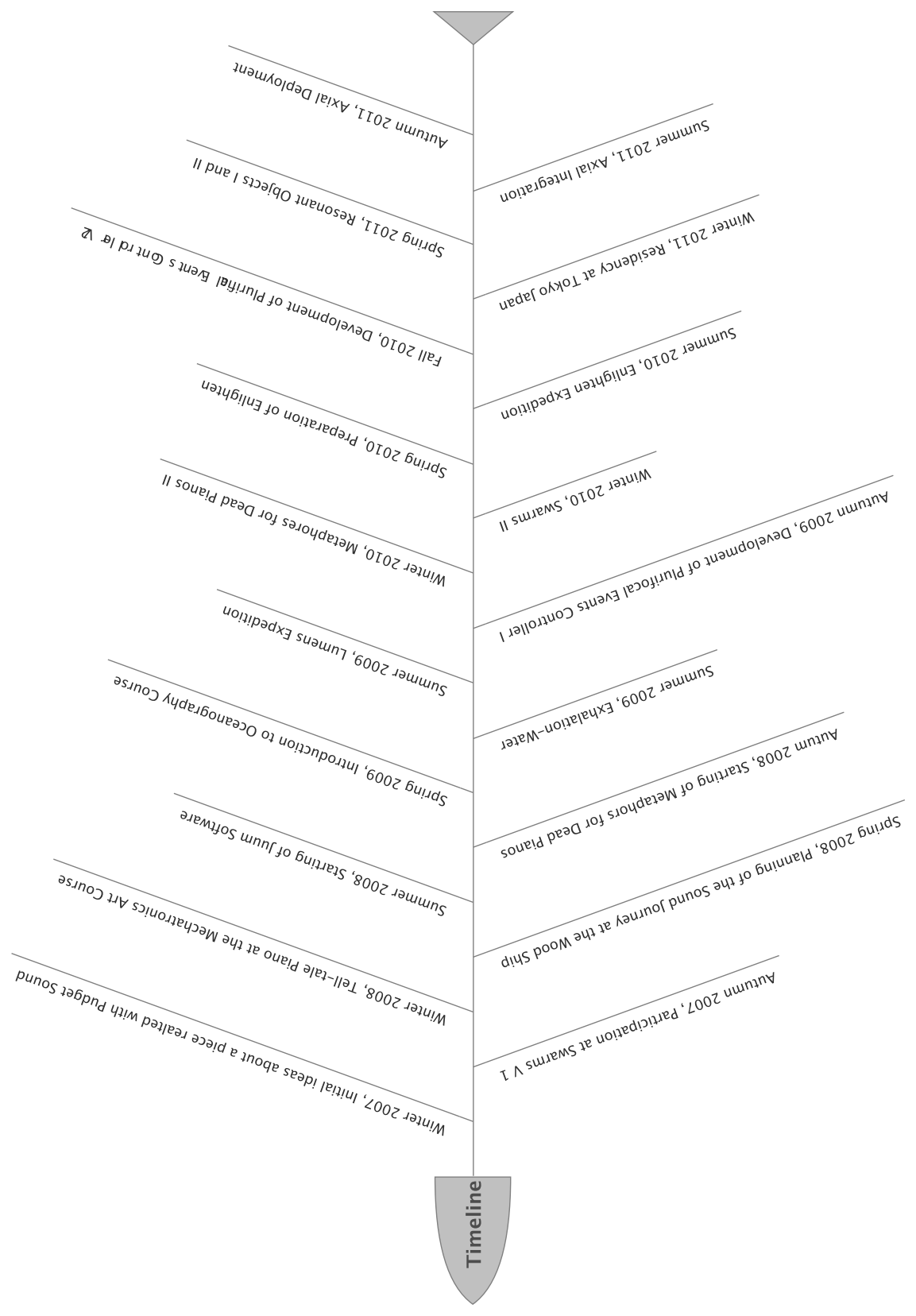

Figure 6.1: Axial's production timeline. 
Axial that helped to develop the artistic resources, and also as a set of logistic decisions. At that point the piece had to be composed and shaped.

Collecting ideas in brainstorm fashion, discarding some of them, modifying others, and confronting the benefits and issues of each remaining idea was a natural process realized over several iterations by drawing and sketching (see figure 6.2). Although the sonic and conceptual component was the guidance for the artistic decision making, the path that the piece followed was a mixture of artistic, logistic, and technical compromises. With this heuristic evaluation technique, the piece was gradually shaped and after three months achieved its final status.

\subsubsection{Planning of the Physical Manifestation}

The starting points for materializing the piece were sketches in a notebook drawn while imagining physical possibilities and distribution of elements. An important constraint was knowing in advance the possible location for installing the work. Having rented a ten foot container and having realized a set of experiments and recordings a year before helped to conceived the sound quality and the proportions of the experience. From those tests, the author knew the container would be an extraordinary resonator. At that stage of the creative process it was clear that in order to take advantage of the sonic properties of the container, a large number of solenoids would be spread along the walls of the container. Starting from that logic the focus was on the sonic development.

\subsubsection{Compositional Process of the Sound Material}

The most profound question about the entire project was how the work should sound. Sound would have to be the guidance and the core element. Sound should transmit the logic and the essence of the sea experience. While the aesthetic had been partially set up since the beginning, following on the conceptualization, design, production, and realization of previous projects, when this project was started there was not a clear idea about what would be the sonic experience. Once again, a brainstorm method and a set of iterative decisions over several months started to shape a feasible sonic experience.

The starting point was the collection of sound recordings from the ship, specifically the recording of the hydrothermal vents. Also, the author had a set of SuperCollider 


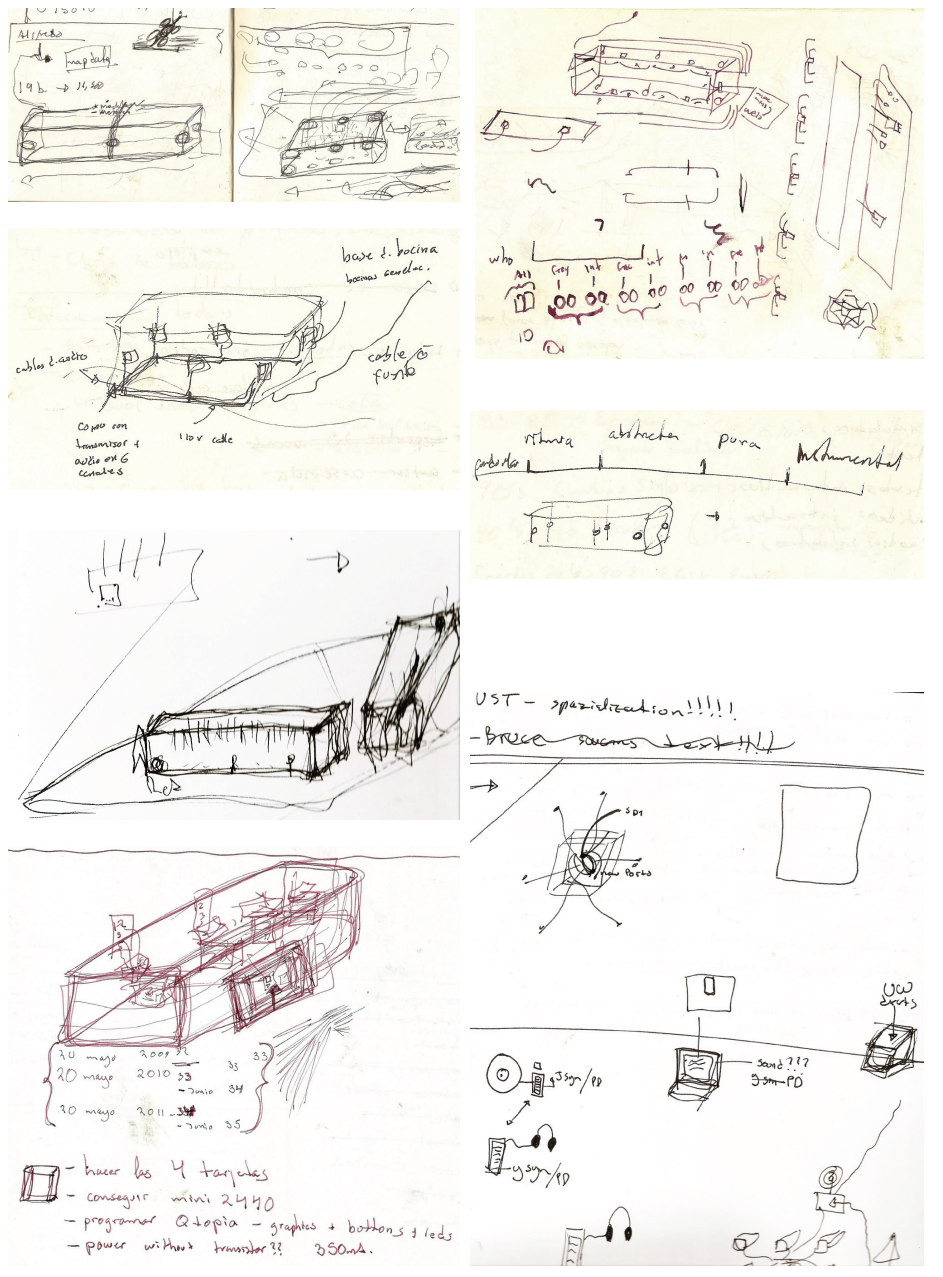

Figure 6.2: Sketches of ideas. 
codes developed during the second expedition that allow me to use scientific data generated by the equipment on the ship as modulators of sonic parameters.

At that point in time, the author had finished a set of works that employed mechanical devices to generate acoustical sounds with digital controls. It was natural to continue with such exploration of acoustic sound textures. The hardware and software was ready to be used for this project and it could be the materialization of a long-term research developed during the doctoral program.

Some of these previous mechanical pieces had an interactive component that enabled modification of structures and sonic elements depending on the physical activity and behaviors of participants. While those projects work well as interactive experiences, on this occasion the author wanted to produce a piece that does not rely on direct interactivity. ${ }^{1}$ The challenge of producing a linear work was attractive and motivating. Within this context, the author decided to work on a piece made of a fixed loop composed of two layers, an acoustic material produced with a mechanical platform and a digital material composed with the sound and sonifications obtained on the ship.

The duration and structure of the work were not determined from the beginning but they were gradually defined by an iterative process of trying, testing, redefining, and reorganization. The Juum software, which is explained in chapter Juum Software, allowed for easy manipulation and reorganization of structures while the Plurifocal Events Controller, which is explained in chapter Digital Plurifocality and the Plurifocal Events Controller allowed for the production of distributed rhythmic patterns. Such patterns can be triggered directly from the Juum timeline, making the exploration and testing process relatively simple.

\section{Compositional Process of the Analog Layer}

Even though the analog and the digital layers were produced and tuned at the same time, with interrelated influence between each other, the analog layer was probably the detonator of the structure and duration of the artwork.

\footnotetext{
${ }^{1}$ The dialogs and conversations with the faculty of the Center for Digital Art and Experimental Media were important for encouraging the production of an artwork that does not require the direct input of the observer.
} 


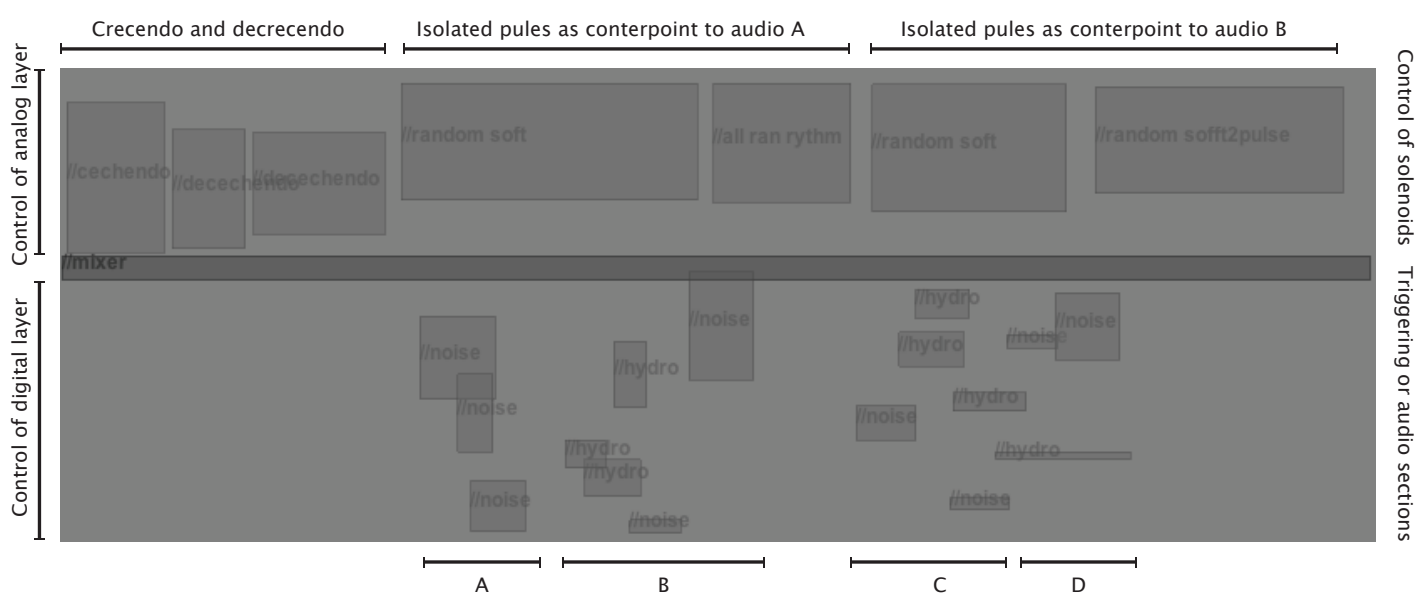

Figure 6.3: Axial score showing layers and sections.

As explained in Fabrication of Mechanical Components, the latest version of the Plurifocal Events Controller allows for the creation and manipulation of polyrhythmic structures spread over multiple sources and locations. The first explorations led to complex structures and intricate textures that resemble some avant garde percussion compositions; however after an initial period of excitement and surprise, the musical gesture started to feel out of place. The author started to feel -or hear, we should saythat the traditional musical logic was interfering more than supporting the core logic of the piece. After all, this work is not a musical composition, but a sonic experience with other kinds of relationships between time, sound, listeners, and physical space. More than ever, the author felt and lived the difference between the musical tradition and the concept of sound art.

With each iteration of the compositional process, the initial polyrhythmic complexity became more and more thin and direct. A decantation process emerged as a mechanism to homologize the core idea of the piece. The complexity was at the end transformed into a single gesture that translated better the logic of oceanographic processes. The time period of oceanographic processes are extremely long, transformations are slow and gradual, but inevitably notable in the long term. The sound logic should reveal that logic. 
Between the initial sketches and the final version of the work almost a year passed. During that period a dramatic event took place that helped to solidify the compositional structure of the piece. In April 2011, the Axial Volcano erupted, fulfilling the prediction that it would erupt before 2014. The event was carefully followed by the oceanography community and it was extremely important for the Ocean Observatory Initiative since Axial is located in the area of study. The eruption was not monitored and the opportunity to register the event was lost since the equipment of the OOI was not installed yet.

The April 2011 Axial eruption is a demonstration of the importance of transmitting seismic, video, and vent fluid chemistry information in real time. The eruptive event, or series of events, in April 2011 went undetected until a series of ROV/AUV cruises, planned separately and years in advance, made observations to discover, confirm, and detail the transformation to Axial (see Chadwick et al., this session). What transpired during and shortly after the eruption will remain largely unknown, as the initial extraordinary fluxes of heat, chemistry, and biology will have decayed in the intervening three and a half months.[32]

The author was at that moment working on pieces that used mechanical devices to produce loud masses of sound. Imagining the gradual accumulation of energy and its liberation triggered what would became the final structure and gesture of the work. After the event, the author visualized an overwhelming and direct gesture as the shape that could tie together all the different pieces that constitute the work. The unrecorded eruption of the Axial Volcano became the narrative structure of the piece and after that decision was made, the rest of the compositional process flowed naturally.

Over several days, the author played with different algorithms that could generate and control the rhythmic patterns that the Plurifocal boards could generate. Again, an iterative process of trying, listening, and modifying led to the rhythmic cell that generates the gradual accumulation. The patterns and the duration of each event was graphically controlled with the timeline of the Juum software.

The following code belongs to the crescendo element of the Juum score. 


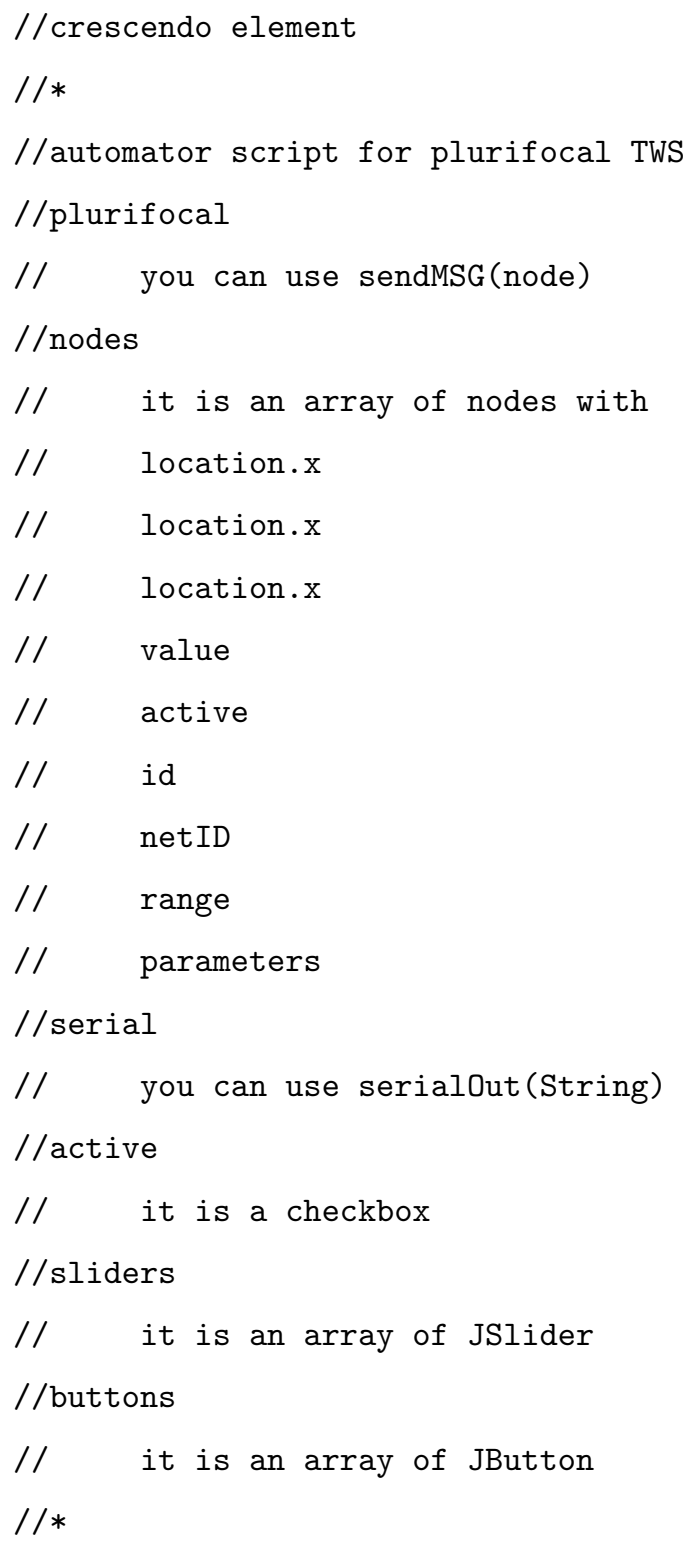




\section{//ACTIONS}

void boardsSet(intensity, period) \{

String intS = Integer.toString(intensity, 16)

String perS = Integer.toString(period, 16)

serial.serialOut("\$address FF 00 \$intS \$perS $N \backslash n \backslash r "$ )

\}

void motorSetAll (board, motor, intensity, period) \{

String brdS = Integer.toString(boardsIDs [board], 16)

String mtrS = Integer.toString(motor, 16)

String intS = Integer.toString(intensity, 16)

String perS = Integer.toString(period, 16)

serial.serialout ("\$address \$brdS 01 \$mtrS \$intS \$perS $N \backslash n \backslash r "$ )

\}

void motorSetIntensity(board, motor, intensity) \{

String brdS = Integer.toString(boardsIDs [board], 16)

String mtrS = Integer.toString (motor, 16)

String intS = Integer.toString(intensity, 16)

serial.serialOut("\$address \$brdS 02 \$mtrS \$intS $N \backslash n \backslash r "$ )

\}

void motorSetPeriod(board, motor, period) \{

String brdS = Integer.toString(boardsIDs [board], 16)

String mtrS = Integer.toString(motor, 16)

String perS = Integer.toString(period, 16)

serial.serialOut("\$address \$brdS 03 \$mtrS \$perS $N \backslash n \backslash r "$ )

\}

void boardsSync() \{

serial.serialout ("\$address FF $04 \quad \mathrm{~N} \backslash \mathrm{n} \backslash r$ ")

\}

void boardsOff() \{

serial.serialOut ("\$address FF $05 \mathrm{~N} \backslash \mathrm{n} \backslash \mathrm{r}$ ) 


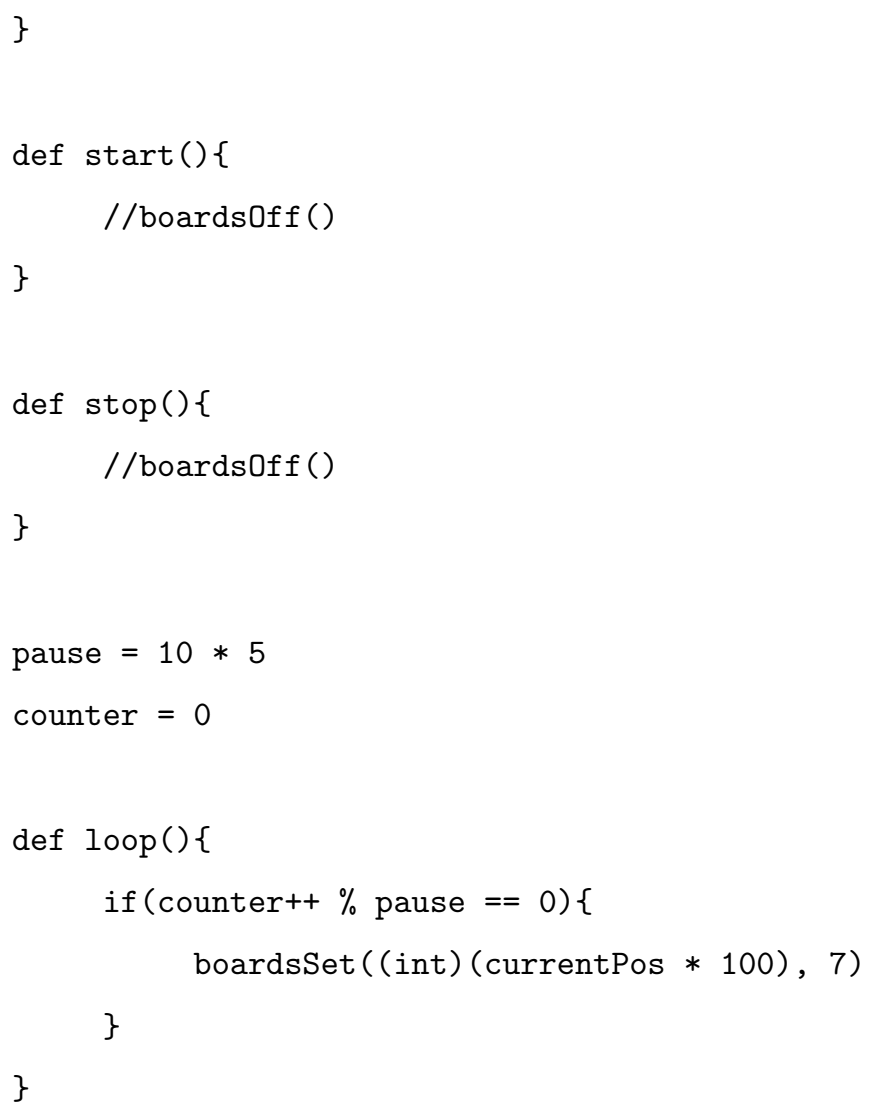

It is possible to see that global variables refers to the modules ID. There is also a set of functions (not used in this particular element) that allows control of the motors by setting the intensity, periodicity, or synchronization. For this particular element the loop function gradually increments the intensity of all the solenoids according to the currentPos variable. For a detailed description of the modules control, refer to Plurifocal Events Controller Specification.

Once the general idea of the piece was achieved and the time phase of the work obtained, the analog part was complemented with digital material.

\section{Compositional Process of the Digital Layer}

As described in chapter Preliminary Work, during the expedition to Axial in August 2011 the author started to develop a set of SuperCollider scripts that would allow the transformation of sound recordings and also a set of codes that allows the modulation of sonic parameters using data collected by the scientific party. With these techniques 
the author realized a set of short duration sound pieces. These audio files should be considered tests and explorations more than a finished composition, but they generated a small set of interesting and valuable sonic textures. The first set of short fragments were created on a personal computer on board the Thompson vessel.

Between November and December 2011, the architecture for sound rendering on Amazon Cloud was finished, allowing the production of longer textures using a bigger set of data files.

The following Groovy code allows the generation of a SuperCollider score from a Knudsen file. For simplicity, the presented code belongs to the desktop version. Please refer to Cloud Synthesis to see the entire architecture of the Amazon Cloud Synthesis version. The code makes readable a sub-bottom profile file generated by the Knudsen Chirp and maps the density of each physical point into a sound grain. Refer to the Sub-bottom Profiles section to see the description and image of a sub-bottom profile. With this code, and the later rendering of the generated score, what it is produced is a kind of granular synthesis texture where the grain density and frequency are modulated based on the information of the sub-bottom profile. In this way, the geological data is directly used to modulate the sonic parameters of a sound section. The properties of the profile can be clearly heard and sonically appreciated.

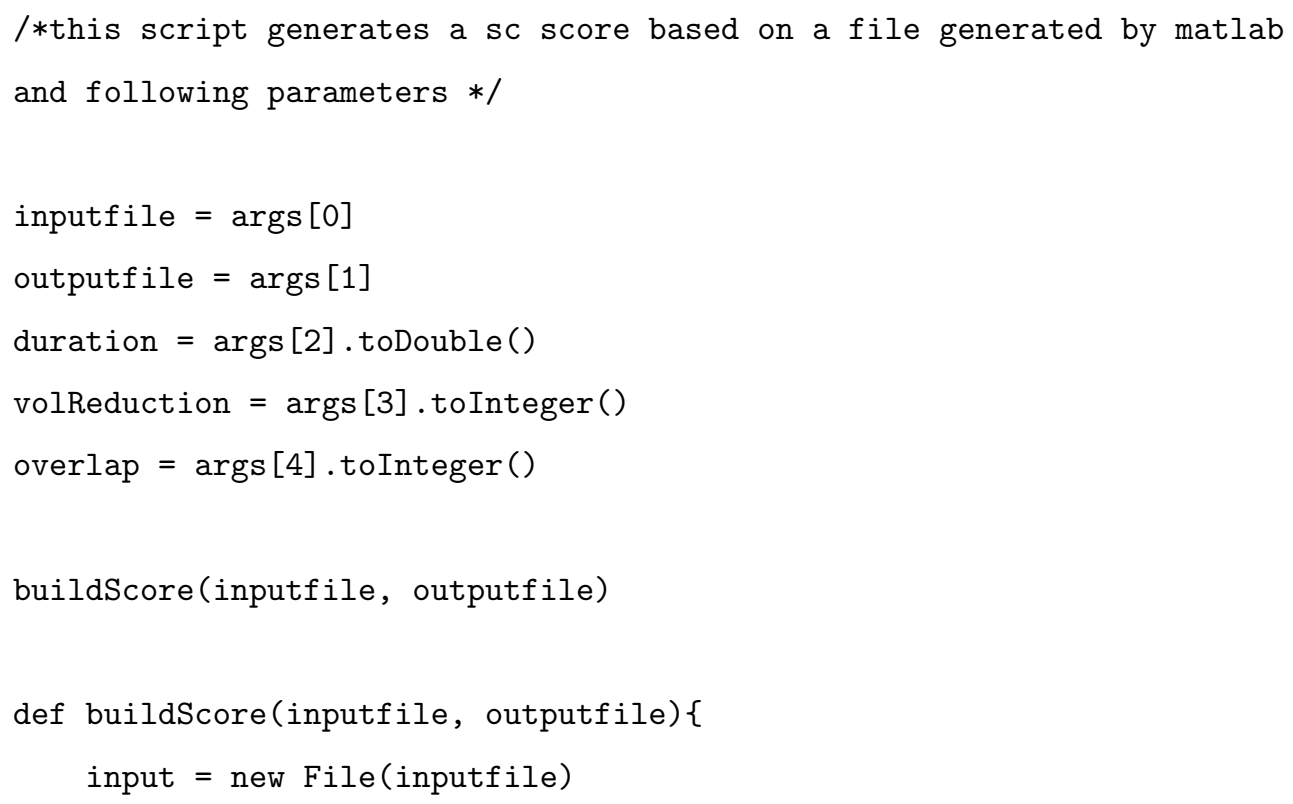




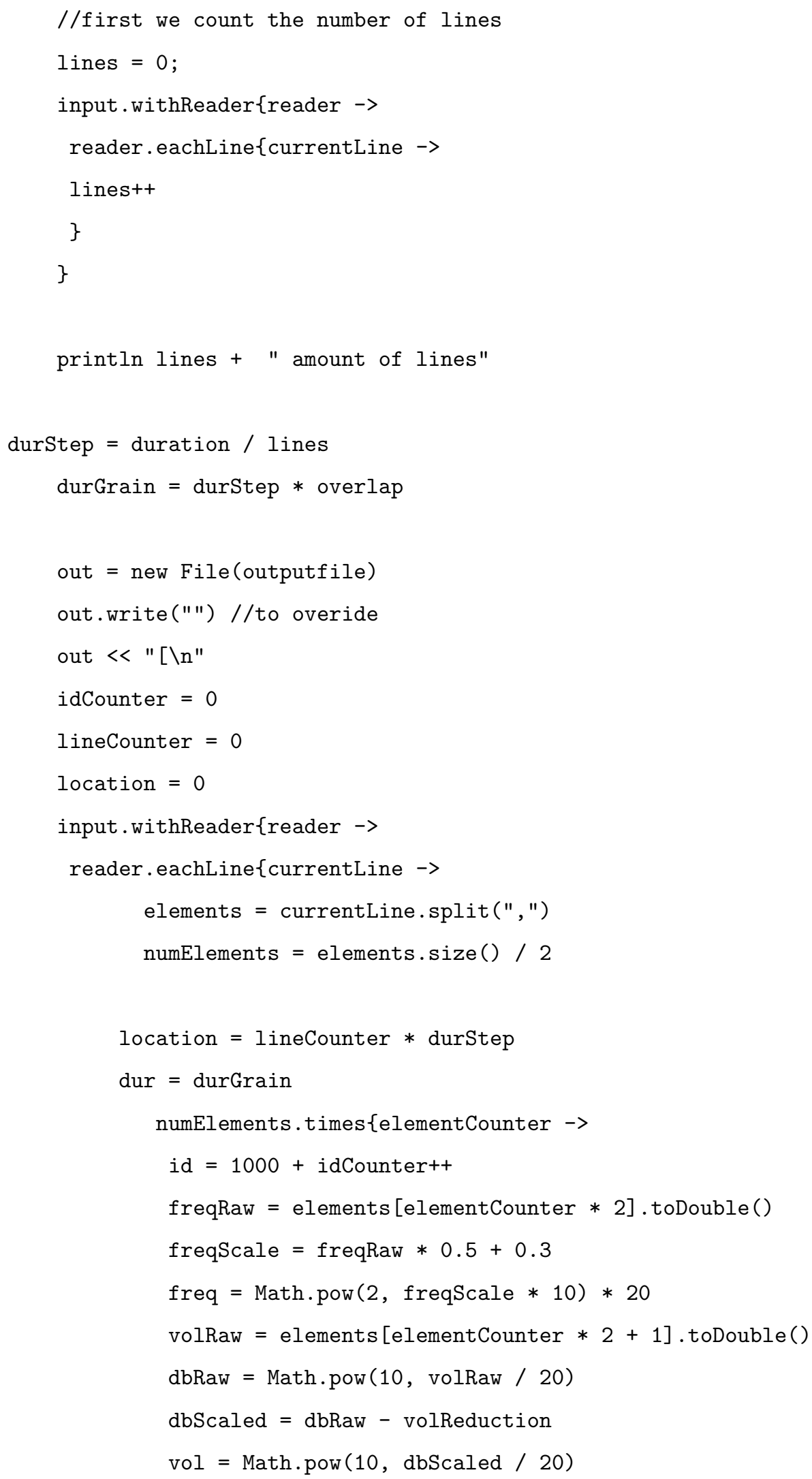




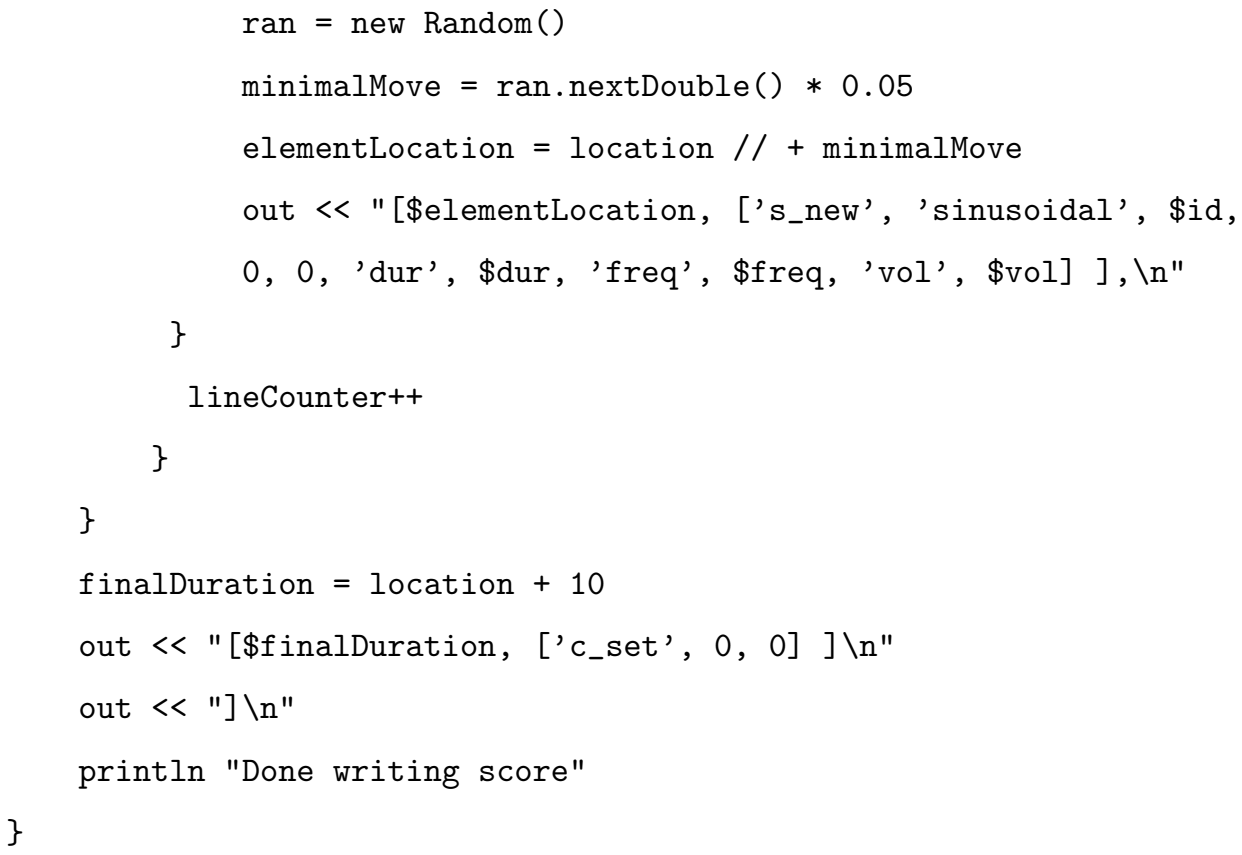

The pool of generated sound was a natural starting point for shaping the digital layer of the sound installation. Therefore, once the global structure of the piece was defined and the analog part sketched, the latter was complemented with these sonic materials. Once again, the Juum timeline helped to organized the sounds by manipulating starting and ending points as well as volumes and Ambisonics distributions. ${ }^{2}$ The organization of materials was produced similarly to the rest of the composition by planning certain heuristic sound distributions, listening, and gradually rearranging elements until the sense of balance was obtained and an attractive dialog between the digital and analog parts was reached. By successive approximations, the digital sound textures were arranged in order to generate a counterpoint with the mechanical pulses.

The compositional process between April and November of 2011 was supported by a digital emulation of the mechanical part and also by a quadraphonic sound system. The final tuning and calibration could not be realized until the physical container was obtained. At the end of November 2011 the container was rented and located on the showing spot.

\footnotetext{
${ }^{2}$ It is important to remember that Juum controls a SuperCollider server for sound generation.
} 
Compositional Process During Installation, Running, and Calibration

Between November 2nd and 7th 2011, the mechanical components were installed in the container. It was not until the installation process started that the decision about where and how to mount the solenoids was made. Being physically inside the container gave a real sense about sound distribution and resonance. Once there, the author decided to evenly distribute the solenoids at ear level and also to hang the speakers from the walls.

After installation was complete, a period of calibration started in which some compositional decisions were made. The specific acoustics of the container and the perceptual changes of being in an obscure and empty container opened a small window for calibration and compositional adjustments. The most significant changes happened at the amplitude levels of the different layers and sonic elements. A final revision of time distributions, pattern speeds, and equalizations also occurred on site during the days before showing the piece to the public.

\subsection{Axial Structure and Parts}

\subsubsection{Physical Manifestation}

The piece is materialized in a 40-foot cargo container. The container is the boundary of the micro-world to be created. It is metaphorically our deep sea and generates a strong border between the inside and the outside. It allows us to generate a dark space and is not only the space where the piece exists but it is the piece and the sound itself. The space is also the resonator and it generates a set of relationships between itself and the outside; between the audience inside and itself. The container is also a metaphor for the ship, which generates a vast void on the outside.

While not totally formalized before the exhibition, the piece generates a dual lecture according to the audience's location. It can be appreciated from the inside, generating an immersive sonic experience, or it could be appreciated from the outside, converting the audience into an observer that finds a planted sonic body in the middle of the void. 


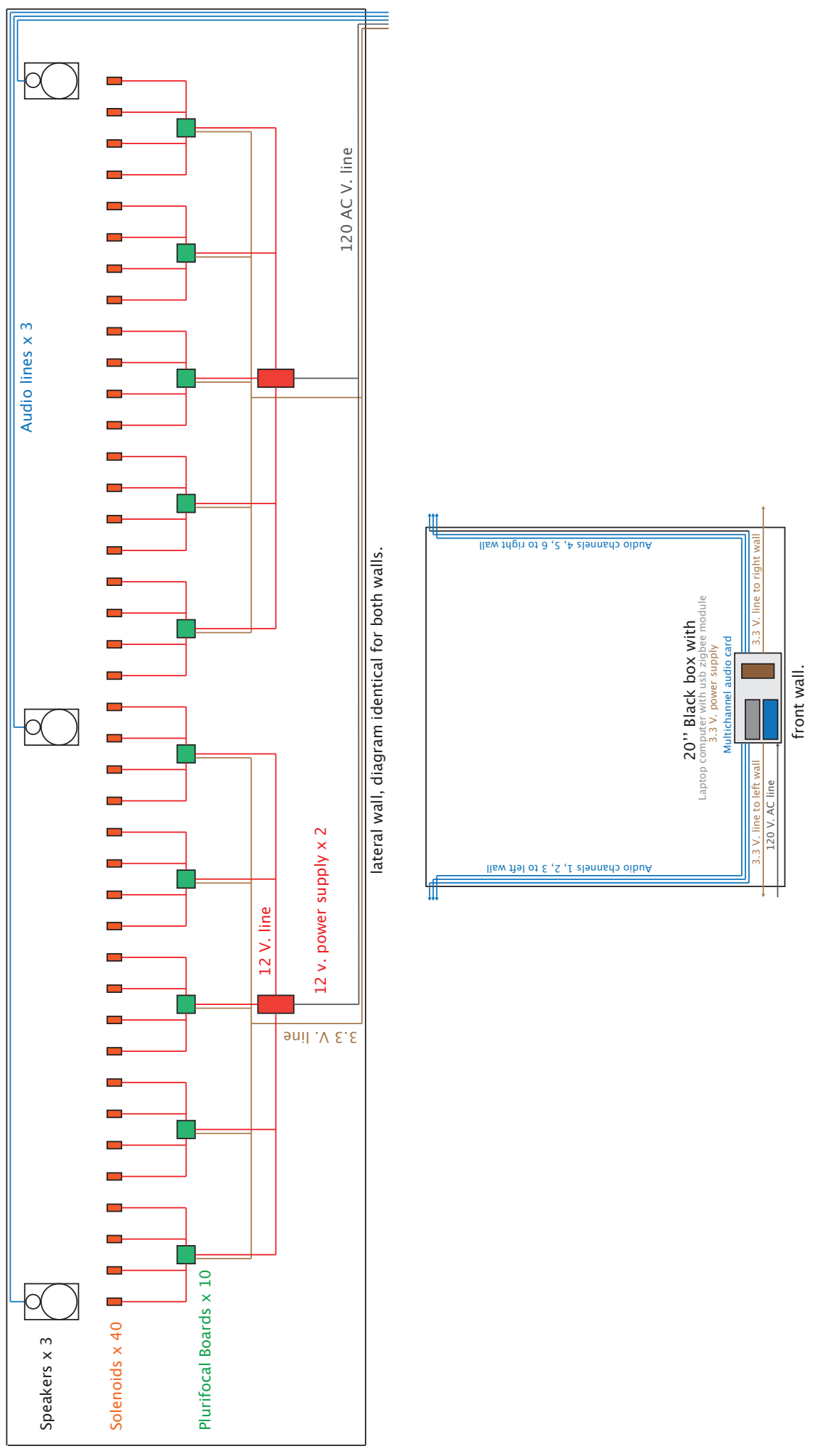

Figure 6.4: Axial's diagram connections and parts, lateral wall and front wall. 


\subsubsection{Compositional Structure}

As mentioned before, the organization of the sonic material did not follow a formal composition or a template in the sense of the traditional musical form. The work was intuitive and based on trying, listening, modification, and tuning of lengths, parts, and the overlap of elements. The composition had to interrelate and create dialog and counterpoint between two kind of materials: the digital layer and the acoustic layer made of percussion sounds produced with mechanical devices. Figure 6.5 presents the sections of both materials and gives the best representation of the compositional structure.

The digital layer starts with a section made of data sonification material followed by a transformation of a ship recording. Later a high frequency hydrothermal section anticipates a long section made of hydrophone recordings that contains sounds of the Jason ROV. After that, the hydrophone recording that has electrical interference anticipates a last version of the hydrophone recording that boosts the low-frequency component. The logic is simple and generate medium-sized periods of tension and calm.

The analog material is made of three sections spread over the piece. The first mechanical intervention is made of regular and fixed pulses. The second part is made of a basic rhythmic pattern that creates two crescendo and decrescendo elements. In this part, the rhythmic pattern is also fixed but has some instability that supports the acoustic dialog with the fourth part of the digital layer. The last analog section constitutes the loudest and most aggressive section of the entire piece. This is the section that simulates the volcanic eruption. It is a long term crescendo of all solenoids synchronized. The acoustic level due to the resonance of the container is extremely loud during this section.

The piece has a duration of 30 minutes and 44 seconds and loops indefinitely during the presentation of the sound installation. Since people are free to enter and leave the container at any time, the logic of the piece is not based on a temporal evolution of sonic ideas. The sonic composition is linked to the spatial relationships and the time organization is secondary and a product of the spatial relationship with the listener. The composition was created with the goal of generating a set of different sonic experiences, with logical transitions. In this way, the overlap between layers and the transitions between sections tends to be smooth with long periods of crossfade and gradual evolutions. 


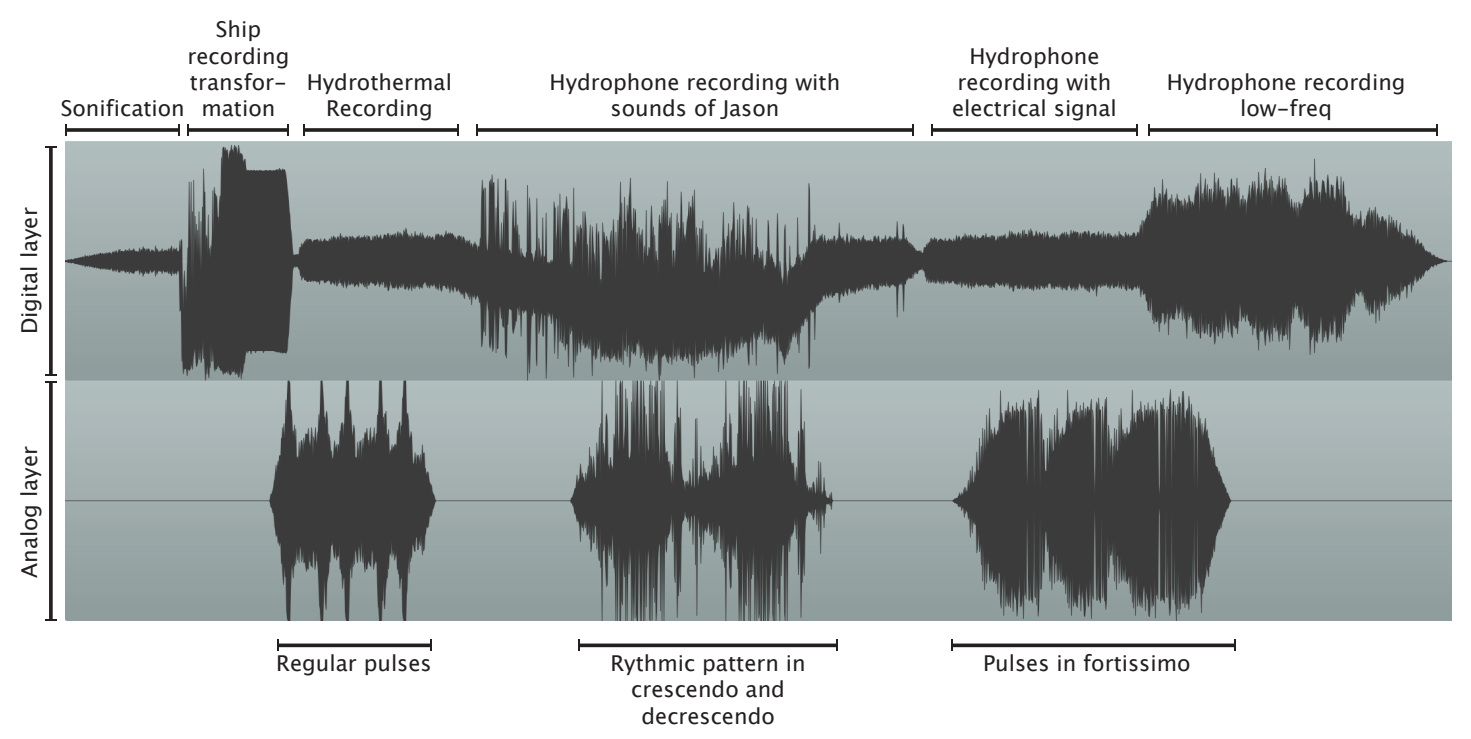

Figure 6.5: Parts and sections of the thirty minute audio loop.

\subsection{Deployment}

As can be seen, the piece was finished in all the different aspects of creation including artistic motivation, hardware and software implementation, and exploration and conceptualization of artistic ideas. The final step in the process for the artistic material was the exhibition to the public. At this point the piece starts its life, it is born when is presented to the audience generating a dialog with the external world.

A set of personal interpretations, analogies, and sometimes unexpected lectures on the work initiates the existence of the artwork. In common with human relationships, artworks in one sense have an intrinsic identity, but are also what audiences, art critics, judges, and a regular audience experiences and describes about the piece. In some way, the cycle is completed only when the piece is experienced by the audience.

\subsubsection{Location}

Up to now, Axial has been experienced by the public once, during the winter of 2011 . This is not a site-specific artwork since it could exist in different locations and situations, but the sheer size of the shipping container necessarily generates a relationship between 


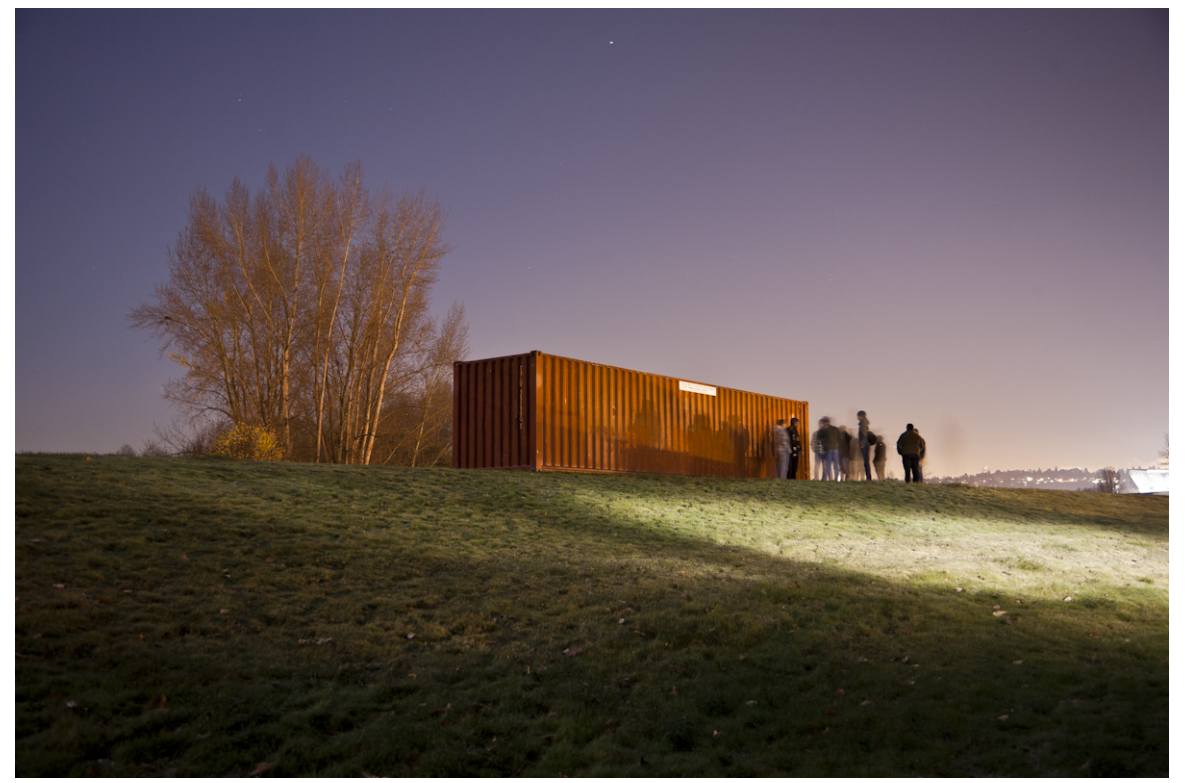

Figure 6.6: Container's view located at the Center for Urban Horticulture at UW. (picture by Daniel Hawkins)

the object and the place where it is located. This relationship is expressed in two aspects: the physical interaction between the container and the place, and the metaphorical meaning of having a shipping container on such location. When it was decided that the output format of the work would be on land, inside a cargo container rather than broadcasted live from the sea as described and detailed in chapter Composition and Deployment, the specific spot had to be decided. A number of locations were considered, among them:

- Fremont DXARTS warehouse

- Cargo and shipping deck of the Oceanography department at UW

- Harbor Island maritime area

- Washington Park Arboretum

- Elliott Bay waterfront 
- Center for Urban Horticulture at UW

Each of the aforementioned places could have generated valuable curatorial relationships with the artwork, and each location required certain logistical issues to be solved. After a natural compromise between feasibility, geographical properties, and the location's meaning, the Center for Urban Horticulture was selected.

The main idea was to select a location in an open space, big enough to plant a full-sized, 40-foot cargo container where the audience would have the feeling of being surrounded by the location. Big enough to perceive the container as small when at a distance and to be able to approach it from different points. The space should be open enough to have a view to the horizon, and silent. The space should work as a reminiscence and analogy of being a ship in the middle of the ocean.

The Center for Urban Horticulture is part of the UW Botanic Gardens. It includes 16 acres of landscaped grounds and 74 acres in the Union Bay Natural Area. It was within this region that the specific spot was selected. The exact location where the container was placed is at latitude $47.659051^{\circ}$, and longitude $-122.291855^{\circ}$. This location faces the UW Ceramic Metal Arts building to the north, UW lands for botanic research to the south, the entrance to the Union Bay Natural Area to the west, and Mary Gates Avenue to the east.

The spot turns out to be ideal for several reasons: it belongs to the UW and so it was relatively easy and economical to rent, it is in a open and silent space, it is near a power line, and it is on a small hill which produces an open viewpoint to the horizon unobstructed by buildings or construction. In addition, the location is accessible by public transportation and also by car, having a small parking lot in the back.

\subsubsection{Setup}

The cargo container was rented from the Aztec Containers Company located in Rochester, Wash. (http://www.azteccontainer.com). The company brought the container by truck and placed it on the right spot and the right direction, orienting the doors to the view of Union Bay. Later, with the support of a volunteer crew, the piece was installed over a period of four days. First, the container was cleaned and the eight speakers 


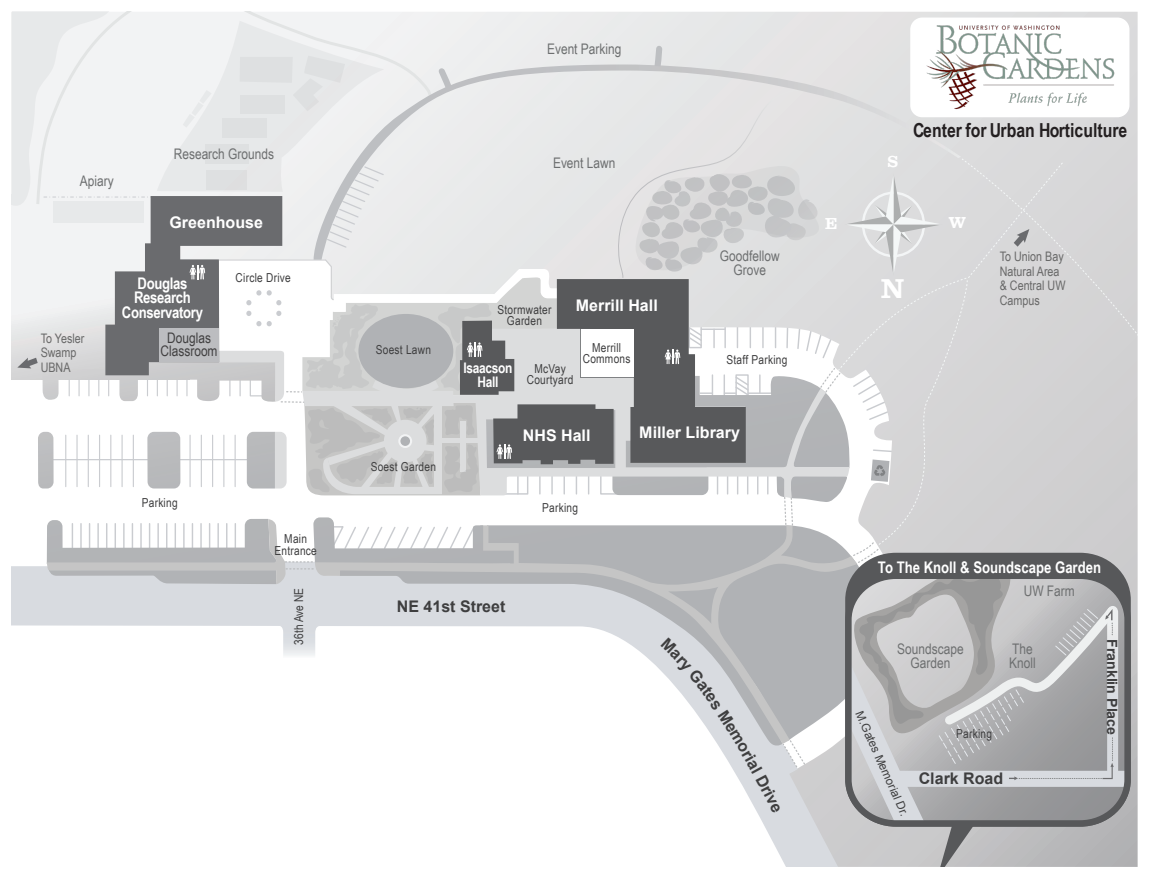

Figure 6.7: Map of the Center for Urban Horticulture.

placed at ear level with air cable. Later the wireless plurifocal control boards and the power supplies were hung with cables. Finally the array of 80 mechanical percussion mechanisms was placed at the same level spread evenly over three inside faces of the container. At the end, the devices were wired to the power supplies. The main power was brought to the container by a power extension cord coming from the art building.

Inside a small black box at the end of the container, a laptop computer was placed. The computer ran the Juum software with the Axial composition.

\subsubsection{Presentation}

Made of two layers, the mechanical and digital, the score looped, indefinitely repeating the 30-minute piece. The work was planned to be experienced inside the container in order to appreciate the mix between the analog material, the digital sounds, and the resonances of the space. The work could be appreciated from dusk to midnight, creating a subtle relationship with the night, enhancing the sound perception and blurring notions 


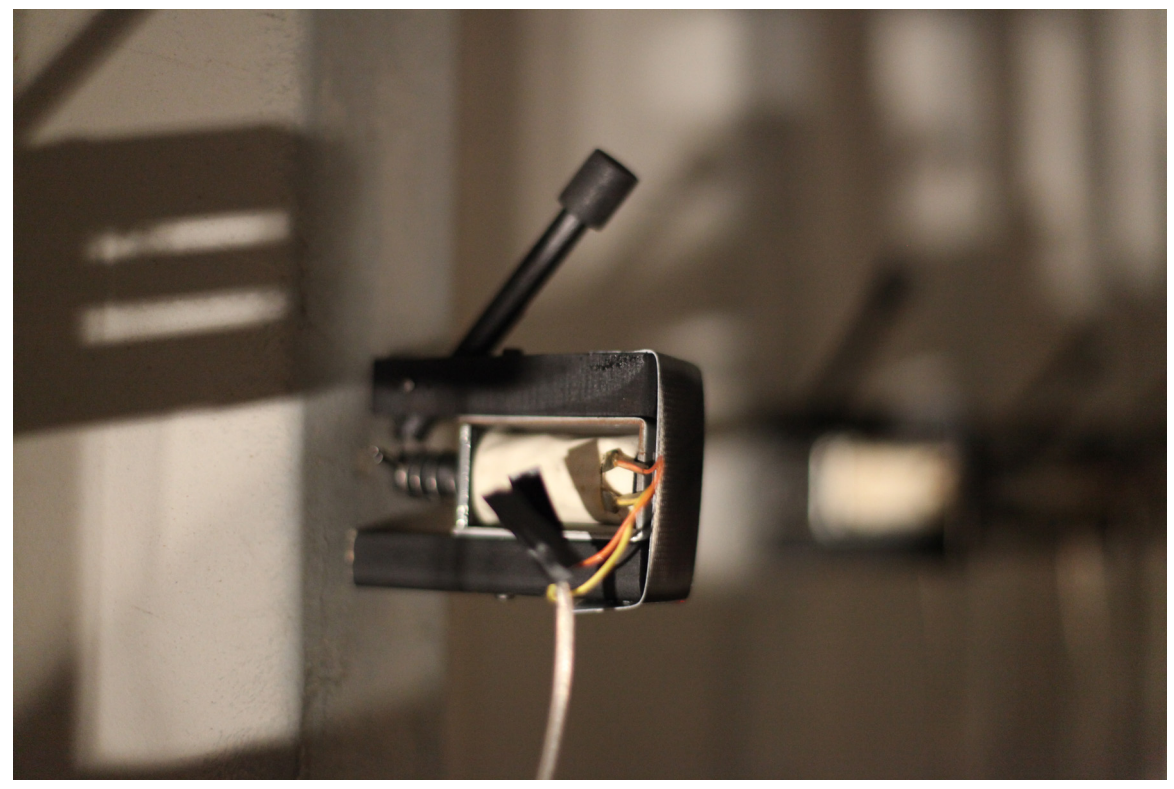

Figure 6.8: Solenoid mounted on the wall of the container.

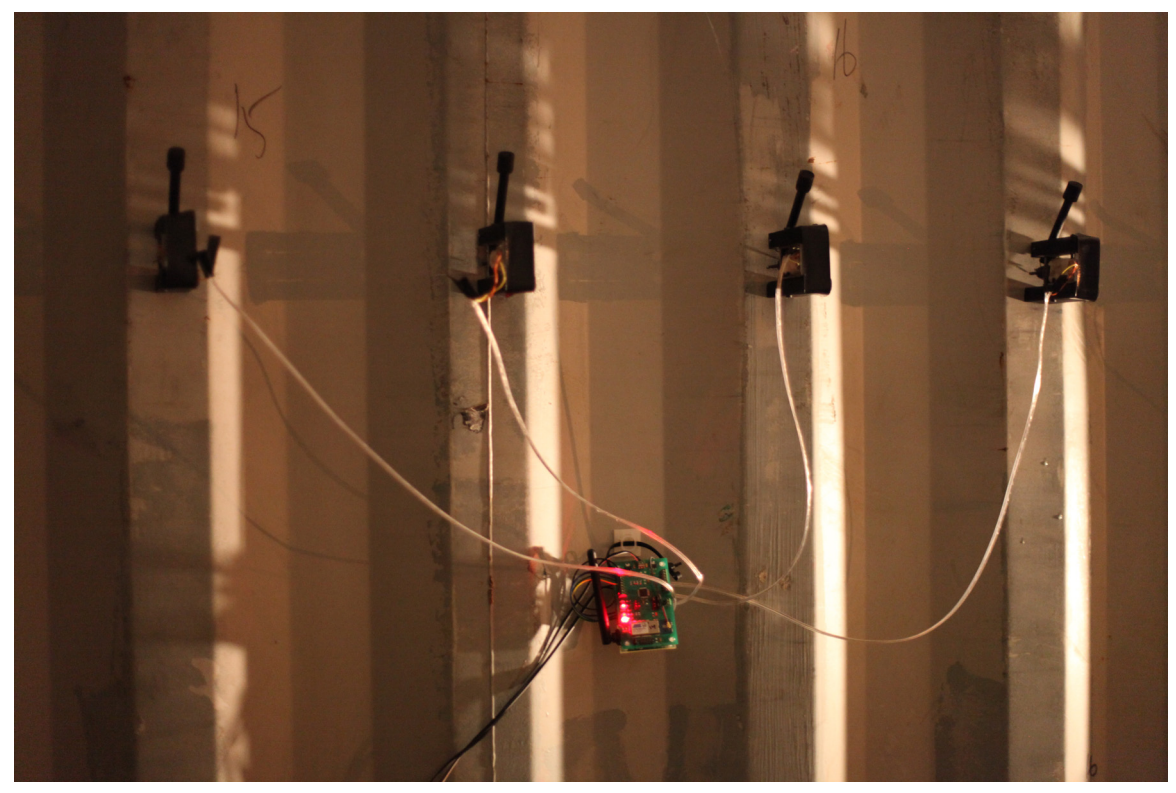

Figure 6.9: Set of four solenoids and one Plurifocal Events Controller. 


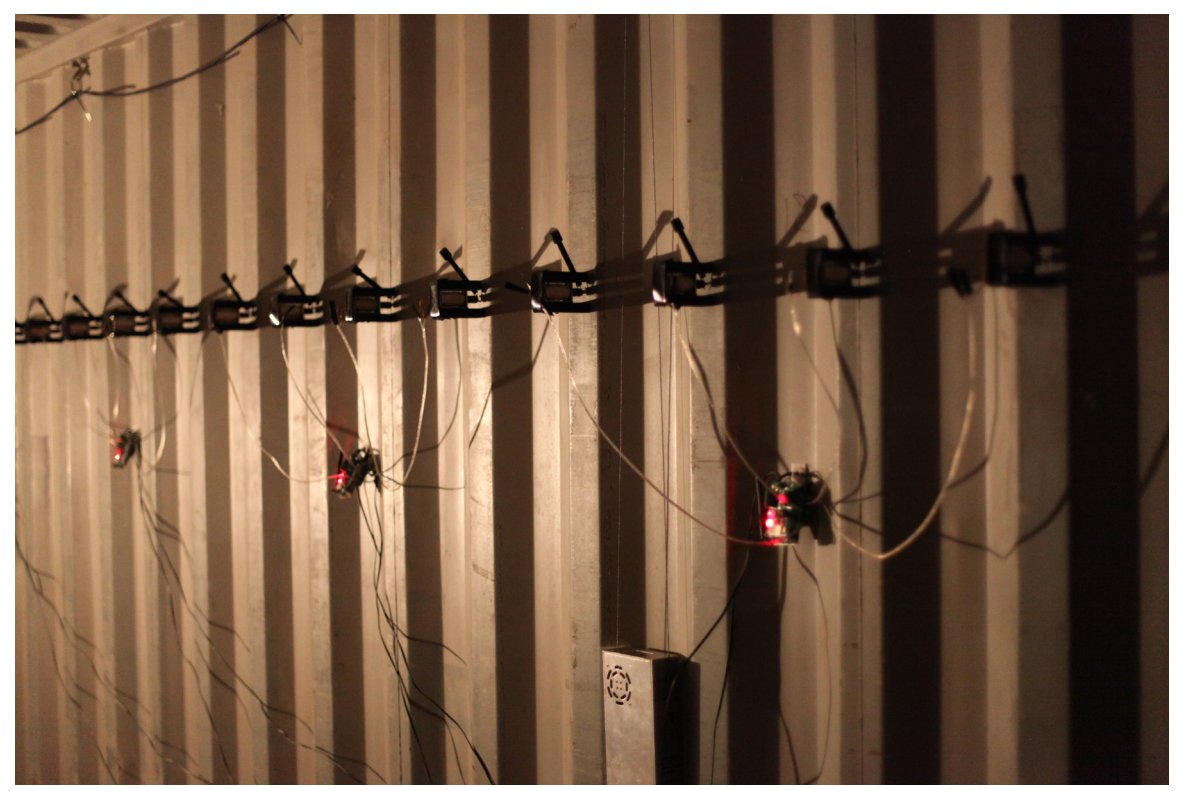

Figure 6.10: Wall of solenoids, PEC, and power supply.

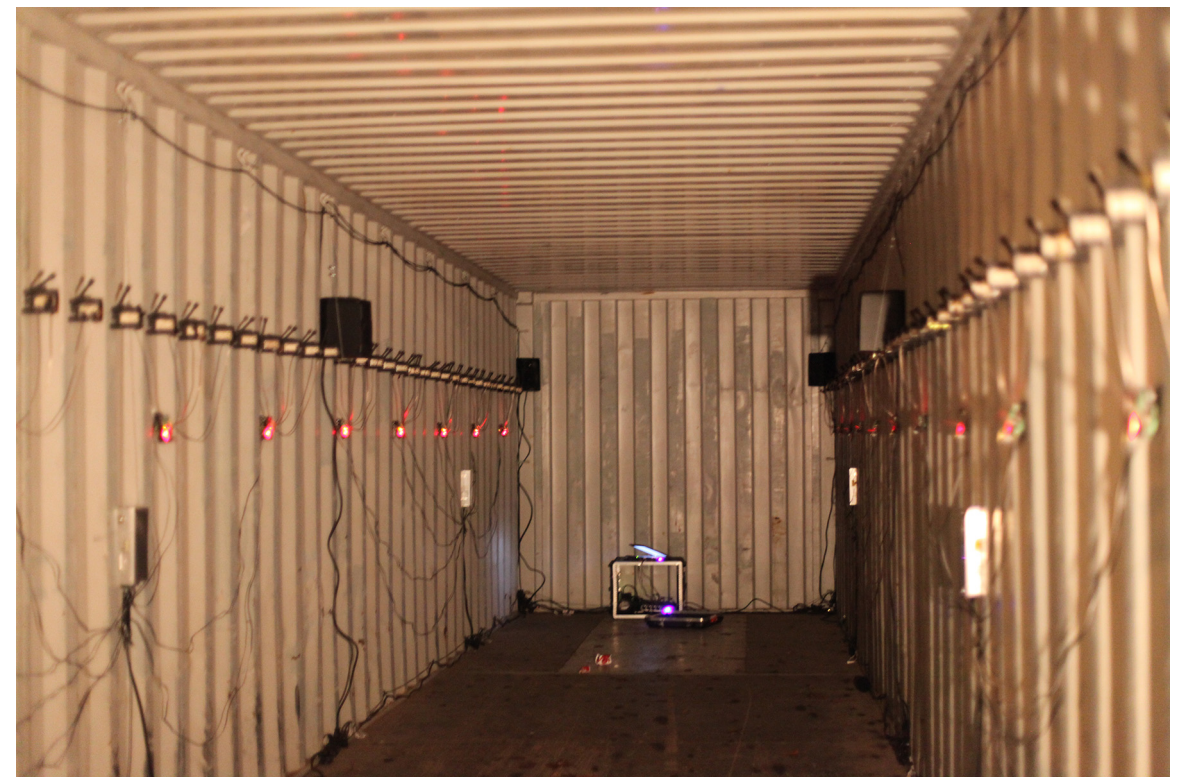

Figure 6.11: Internal setup of Axial. 
of distance and proximity. The two doors of the container were partially open, which allowed a segmented view of the horizon and the entrance of a little light and air into the exterior, and allowed a metaphoric dialog between inside and outside, surface and profundities.

The work was open during two periods of time; on the first occasion it was open only as a preview for the faculty committee, and a month later it was open to the public. Compositionally, the piece was altered very little between these two showings, mainly on the balance between the digital sound and the mechanical layer. Technically the piece reduced the amount of computers used for the execution.

\section{Exhibition for the Committee}

The exhibition for the committee was private in order to generate a space for discussion, analysis, and observations without disturbance. The committee arrived at the location at night, as requested. Bypassing any introduction or preamble, they experienced the piece without any direction or guidance for about an hour. They spend time inside the cargo container but they also walked around it, listening to the merging of different acoustic elements from the outside, from different locations between 10 and 30 meters away.

Once they finished experiencing the work, they generated space for dialog and discussion. First they listened to an explanation by the artist describing the artistic nature of the work and several points about composition, organization, and decision making. Later they shared opinions between them, pointing out observations, ideas, and references. At the end the committee asked questions about the work and made some observations, culminating in a conversation about sound art and sound installations.

\section{Public Exhibition}

A month after the private showing to the committee, the piece was open to the regular public for three days. With about 50 visitors per day, the piece generated valuable interactions with visitors. People remained for 20 minutes on average, listening to the work inside the container, and feeling the vibrations of the place. Some audience members walked around the container, similar to the committee, while one person decided 


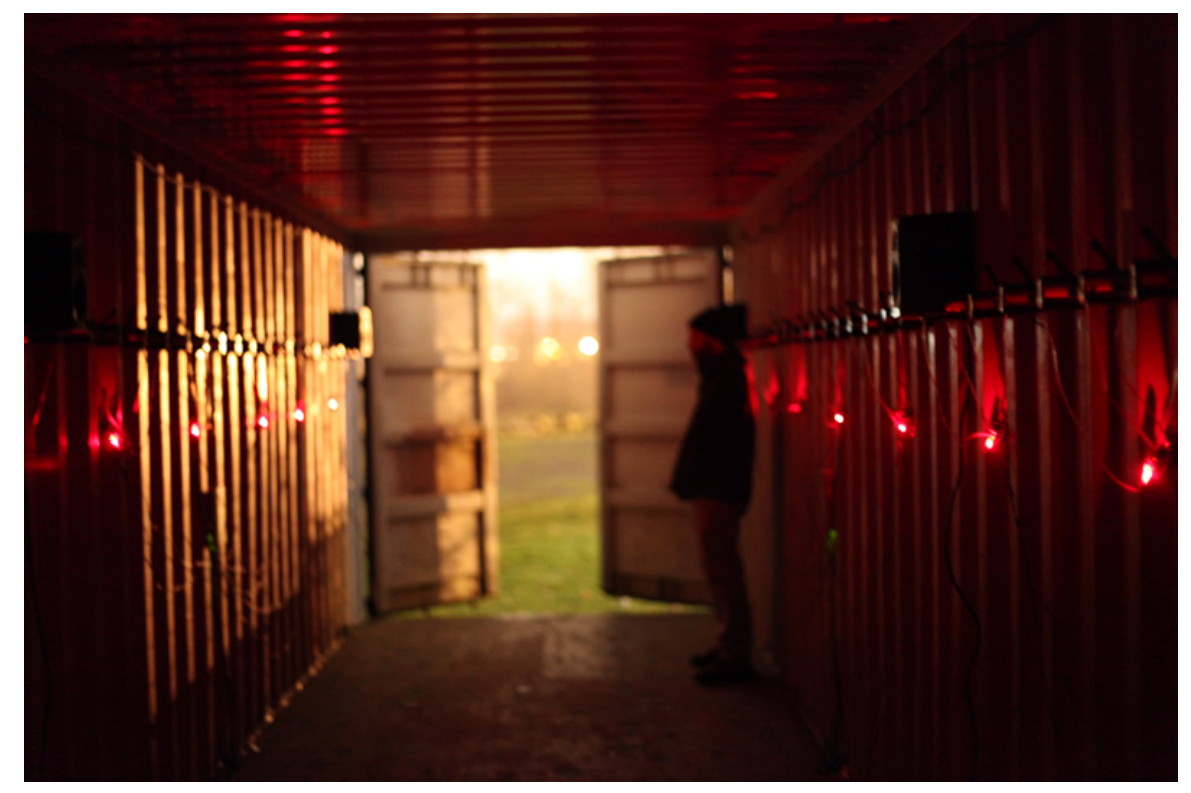

Figure 6.12: Inside view of Axial installation (A).

to experience the work from the top of the container and so climbed onto the roof.

\section{Artist Talks}

The opening of the public exhibition was complemented with an artist talk where the artist gave an overview of the project, including the work and research at the ship, the process of recording the hydrothermal vents, the development of the Plurifocal Events Controller technology, and the translation of the idea to the cargo container on land. About 50 persons attended the talk at the Laurel Village Center, located just across the street from the cargo container.

\subsubsection{Feedback and Comments}

Many of the visitors belonged to the oceanography community. Some of them expressed the feeling that the installation evoked the sea. Although a literal representation was not the artist's intent, it still was interesting and rewarding to have such feedback.

One of the committee members mentioned the work resembles the work of Richard Serra. The mechanical art pioneer Trimpin also visited the work and mentioned that it 


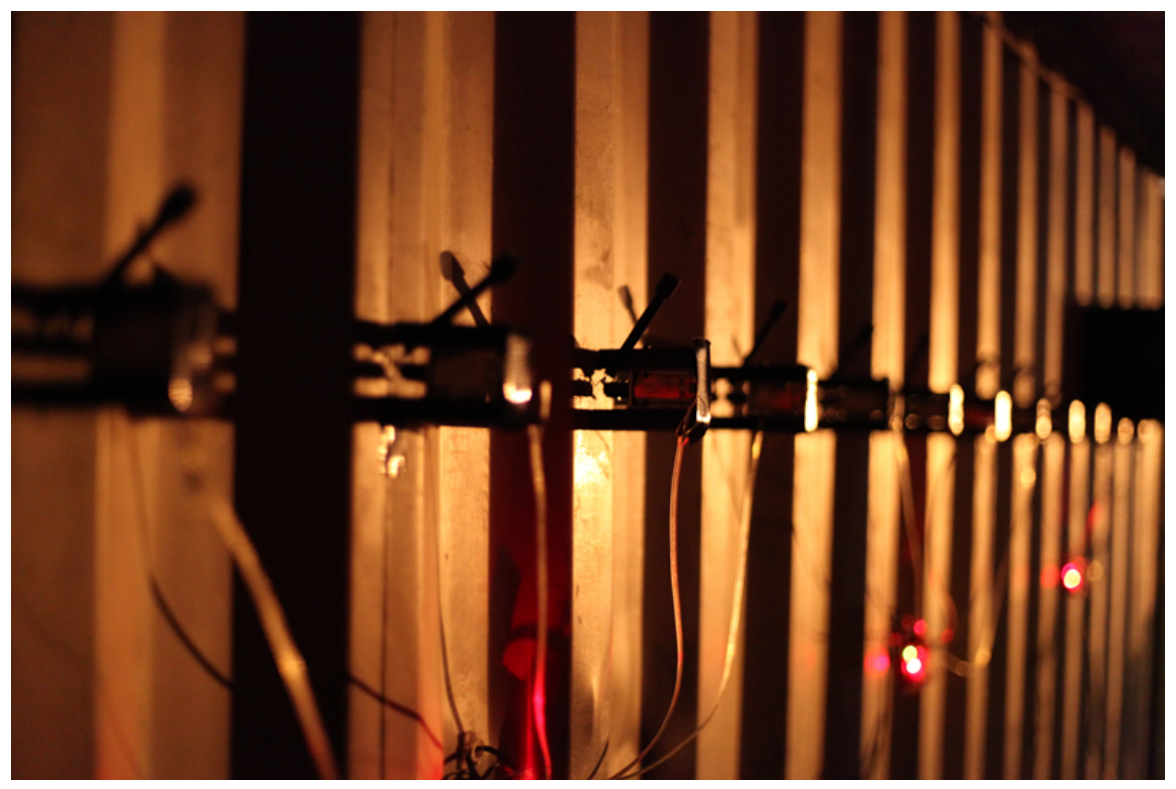

Figure 6.13: Inside view of Axial installation (B).

was inspiring and acoustically interesting. He talked with the artist about percussion controlled by digital devices and how to obtain rich timbre pallets.

\subsection{Chapter Conclusions}

This chapter presented the ideas behind the artwork, some personal ideas about composition and creativity, and the compositional process used during the creation of the work. As can be seen, there is a balance between intuitive decisions, structural and formalized organization, technical constrains, and operational logistics.The different stages of the work and the development of the work swings and orbits between such fields of exploration. This logic is the force that gives momentum to the poetics and aesthetic sub-button profile of Axial. The next chapter gives a general summary of the entire process. 


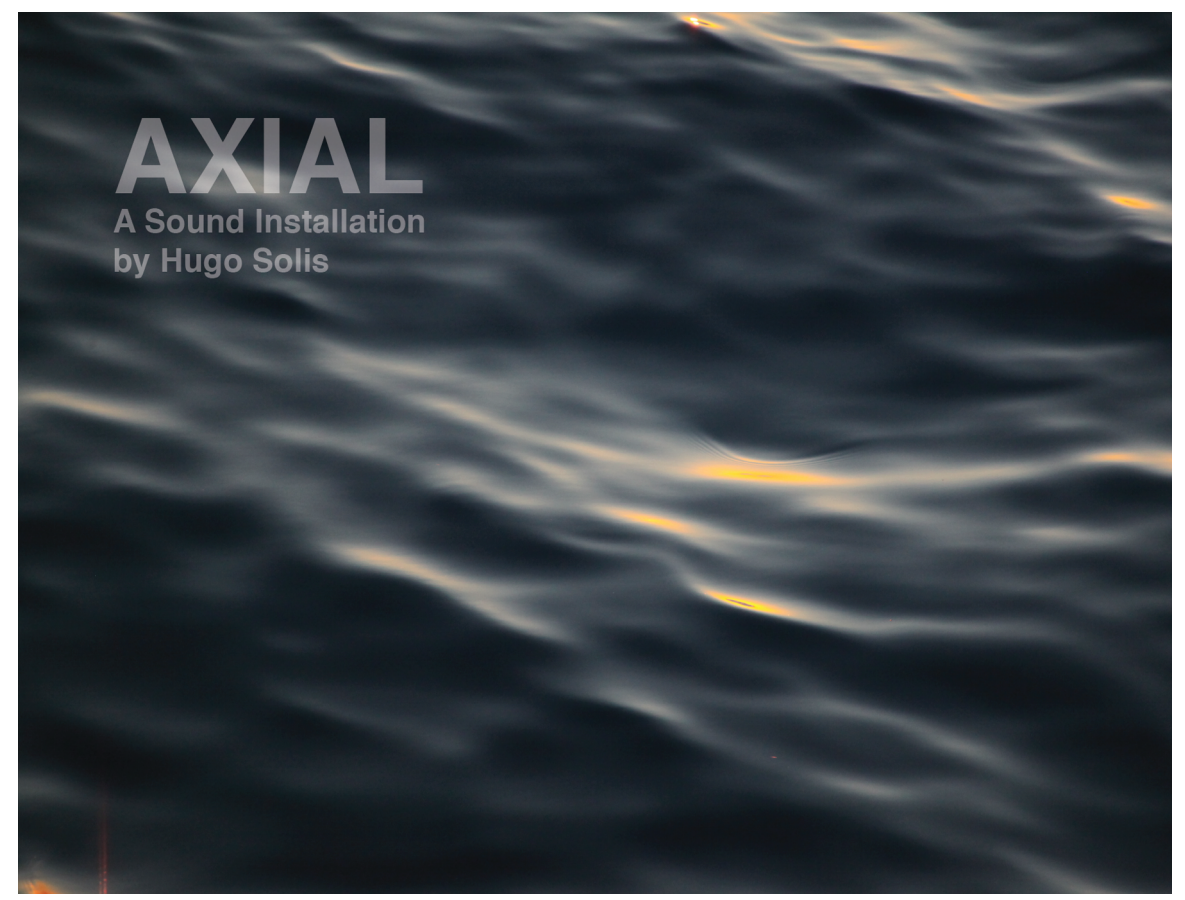

\section{AXIAL \\ A Sound Installation by Hugo Solis \\ December 8th, 9th \& 10th, 2011 Open from 4:18 PM to 11:00 PM}

Axial is a sound installation where a cargo container is $\overline{\text { tol-5 exit } 169}$ employed as a resonant object. Resonant in its acoustic sense because the container is used as sound generator but also resonant in its metaphorical connotation because the container and the generated sounds translate and represent the geological properties of Axial, an active submarine volcano on the Juan de Fuca Ridge located about 250 miles off the coast of Oregon. The container is also the space/instrument for interpreting the scientific data obtained during the oceanographic expedition Enlighten'10 where the artist recorded the sounds of the hydrothermal vents located in the area at depth of over 4500 feet.

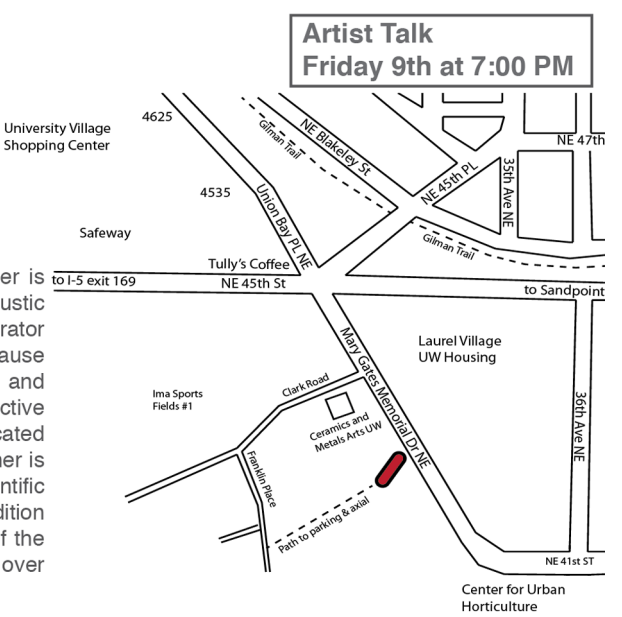

Center for Urban Horticulture I UW Botanic Gardens I 3501 NE 41st St., Seattle, 98195 More info at http://hugosolis.net D:ARTS W UNIYERSITY of
WASHINGTON

Figure 6.14: Postcard invitation to the exhibition and artist talks. 


\section{Chapter 7 \\ CONCLUSIONS AND FUTURE WORK}

"Hace treinta y dos años que estás viendo el mar, Novecento. Desde aquí. Yo quiero verlo desde allí. No es lo mismo"

In Novecento by Alessandro Baricco

This chapter presents the conclusions drawn from having realized Axial and also the future work to be realized in the future as a direct and indirect result of this experience. Since producing Axial was a long term process which required a huge amount of planning, logistics, development, testing, research, and production, different kinds of conclusions are presented.

\subsection{Conclusions}

There is no doubt that the principal reasons for creating Axial were curiosity regarding sound and a desire to explore its potential. The ocean as detonator of images, metaphors, and fantasy permeated the general concept of the work and directed the research and development of the piece.

The sketches and initial experiments started in 2008, which means that it took more than four years to realize the work. Learning about the ocean and the deep sea floor, studying and analyzing previous works with similar concepts, developing the technology both in hardware and software, planning the logistics, creating secondary works where components could be individually tested, and finally presenting and documenting the artwork was a profound and exhausting process with back and forth steps, reversals, errors, and repetitions.

Successive approximations, heuristic evaluations, compromises, trial and error, copying of working models, and repetition of working processes were methods for gradually producing the work. Judging or evaluating the work is perhaps out of the scope of this analysis but it is evident that Axial is not a simple or fleeting work. The challenges, 
the infrastructure, and the required support made Axial a proposal that tries to deeply contribute to the perspectives of sound art.

While several elements could have been achieved better or more directly, the successive approximations that shaped the piece helped to create a wide variety of experimentation, which gradually became narrower until the final version was obtained. Such a method was, arguably, not the ideal means of producing a product, but it was certainly the best circumstance for learning and discovery. Pedagogically, the production of this work gave the author an enormous set of resources and skills for future art creation.

As with any artwork and any aesthetic proposal, this work derives from a body of previous works and proposals created by other artists. The work strives to combine specific aspects of these in order to generate an original proposal, with the understanding

that originality comes from evolution and not from spontaneous generation. Writing about the finished product has provided the opportunity to analyze the evolution of the work, and also to point out the good as well as the less fortunate decisions made along the way. As with any human endeavor, this work is subject to improvements, but what shape they might take can be ascertained only with the perspective gained from the passage of time.

The experience of creating Axial provided expertise and knowledge in the field of sound-art technology and also resulted in a set of mechanisms and processes that future artists may apply to their own creative endeavors.

\subsection{Contributions}

Axial contributes to the field of sound art and electronic art in several different ways:

- The piece as a finished work, with its internal nature, paradigm, and concept, adds to the list of works that explore sound in relationship with space and public spaces. It also enlarges the list of works that have the ocean as main character, creating a dialog with the human and human proportion.

- The work is also a case study of sound and space. Based on the works and concepts of several artists including Pampin, Karpen, and Barber, among others, this work 
explores the logics and nuances of sound and space not only in the acoustical sense but also in the conceptual and social sense.

- The work offers also a set of sound recordings of a location on earth that had never been recorded from a purely artistic perspective. This work and the recordings of the hydrothermal vents work as a manifest of sound curiosity and exploration.

- A technical contribution of this work is the creation of the Plurifocal Events Controller, which offers a solution to analog sound distribution. While there are several mediums to solve this situation, the PEC condenses the process because it was specifically created with this goal in mind.

- Another technical contribution is the creation of the Juum software, which facilitates the time control of hybrid works that require the use of a structured and extended timeline for controlling scripting pieces of code.

- A third technical contribution is the creation of a software infrastructure that offers a solution to the rendering of audio scores. This solution, which uses the cloud computing paradigm, can be easily extended if a large amount of data needs to be processed.

\subsection{Future Work}

The following two projects are current works that are related in some way or another to Axial. The relationship can be in the conceptual, implementation, or technological aspects. These works have had a direct influence on the author's doctoral work and can be seen as a continuation of the research path started as a student. Some are individual works and others are collaborations with other artists. Additional works that do not have a direct relationship to Axial are described on the artist's web page (www. HugoSolis.net).

\subsubsection{Delta 16.57/-99.00}

Delta 16.57/-99.00 is a year-long project with the goal of analyzing and studying the possible, visible, and hidden relationships between sound, nature, water, sustainability, 


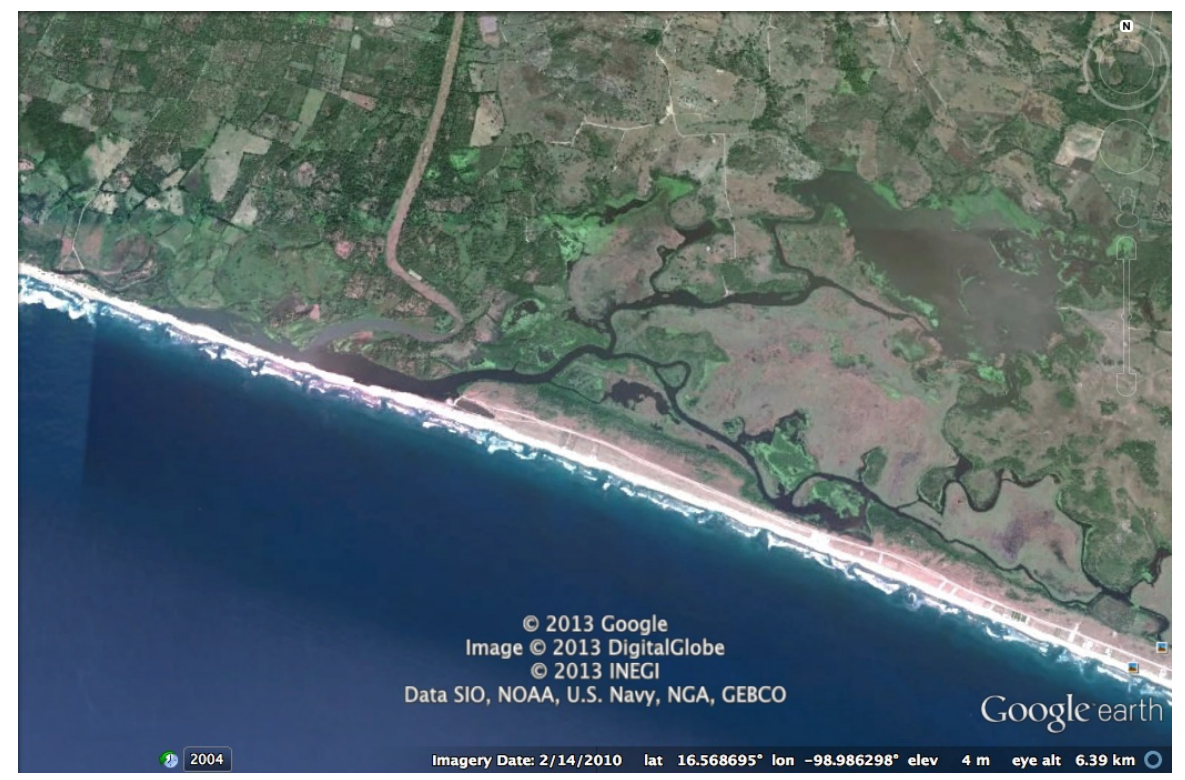

Figure 7.1: Delta location. Aerial image of the Copala River.

and rural elements ${ }^{1}$. On the coast of Guerrero, 120 kilometers south of Acapulco, the Copala River flows into the Pacific Ocean. It is a zone of small rivers and freshwater ponds. There is a delta that opens during the summer and allows the flow of water from the Copala River to the ocean. In the area is a town of about 1000 inhabitants working in agriculture, fishing, and animal husbandry.

This project establishes a dialog between nature, humanity, and technology under several perspectives within the field of land art[25]. It is a hybrid work in the sense that it is a sound installation, a land art project, and also a workshop for exploring the topics of sustainability and art. Two questions form the core logic of the project: Could a technological platform with a nonintrusive infrastructure based on alternative energy generate the poetics and the imagery for a sound work in a rural village, with sound and environmental data as source materials? Could a technological artwork become a cultural gesture that resonates with the use of alternative energy, and being at the same time functional to a rural community that usually uses information technology only as

\footnotetext{
${ }^{1}$ Delta 16.57/-99.00 has received the support of the Programa de Fomento a Proyectos y Coinversiones Culturales 2013 of CONACULTA.
} 


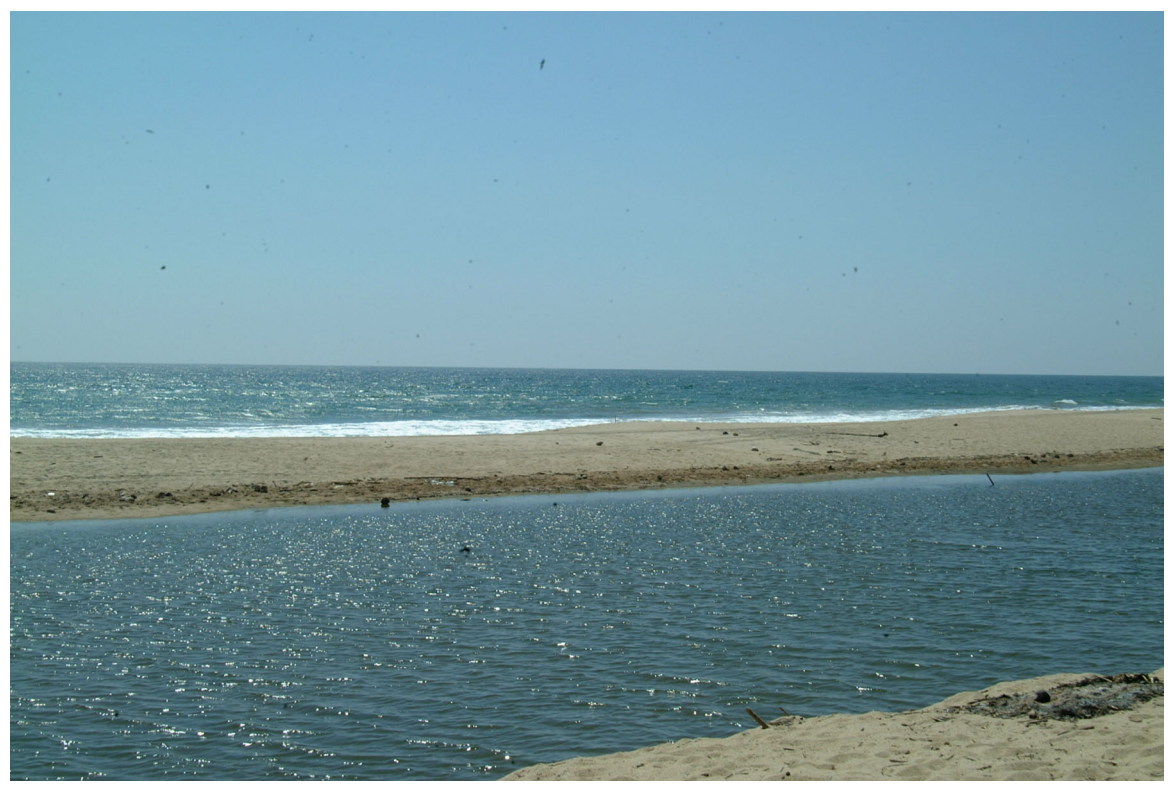

Figure 7.2: Sea and fresh water at the Copala delta.

entertainment?

Just in front of the beach, there is a $80 \times 20$ piece of land that currently is nothing but sand. About 200 meters away there is a freshwater pond and $2 \mathrm{~km}$ away is the delta of the river. The project consists of building a structure on this piece of land that can function as a sound sculpture as well as a central data node. This sculpture will be made of ecological and sustainable materials, and will work also as a shade structure and a site for workshops and courses offered to young people in the area.

In addition, a set of sensor boxes will be designed and built that can measure temperature, humidity, wind speed, pressure, and other meteorological information. These boxes will use solar energy and will create a wireless network sending the collected data to the central sculpture. Since the project is just starting, at the moment of writing several questions are open and will be clarified while the creative process continues. Among the questions to answer:

- What will be the form, size, and materials of the central node?

- What will be the correlation between the registered data and the sound material? 


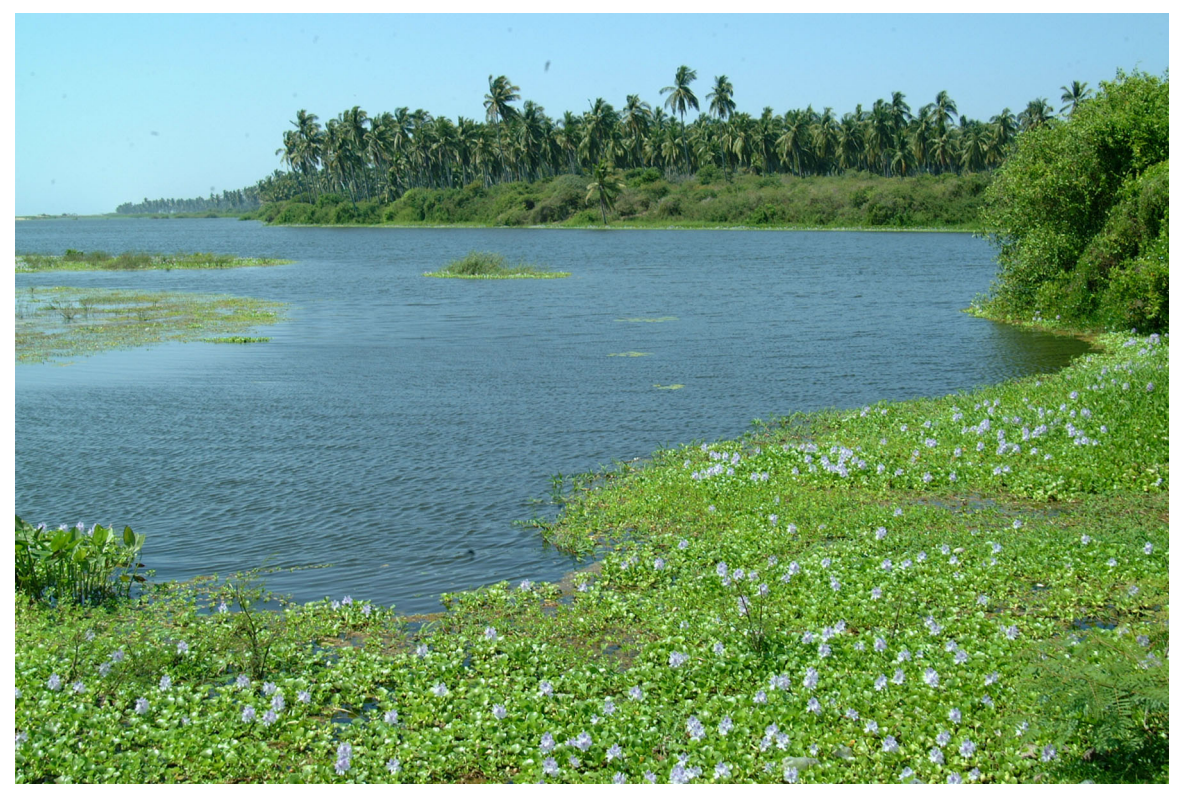

Figure 7.3: Copala River.

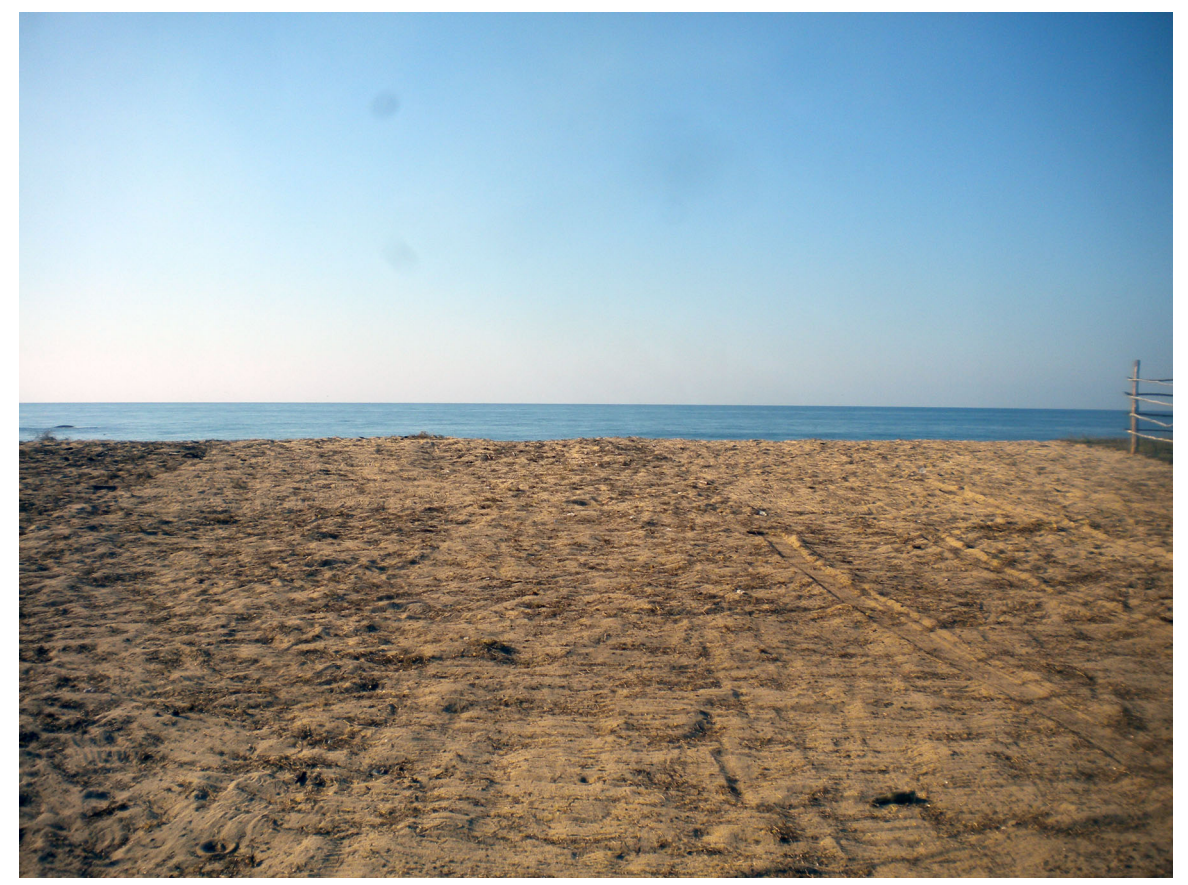

Figure 7.4: Location for the Delta main node. 
- Which medium will generate the sound?

As can be seen from this description of the Delta project, there is a direct connection between Axial and this work in progress. The central use of water, data sonification, and the communion between nature, sound, and technology make this new work a continuation and evolution of the Axial concepts and paradigms. While Axial explored the deep sea as a trigger of metaphors related to unknowns and distances, Delta explores the nearby rural region as a trigger of communion and social integration. Therefore there is a kind of tropicalization of ideas, concepts, and resources.

\subsection{2 $A T L$}

ATL, which means water in Nahuatl, is an abstract narration of Mexico's unique historical journey that goes from Aztec Tenochtitlan to contemporary Mexico. The project will portray social, symbolic, historical, and cultural aspects of one of the largest, most populated, and ecologically most affected cities in the world using water as narrative conductor. ATL is a multimedia experience with water as main character. It is a collaboration with the Mexican musician Alejandro Escuer.

One output of the project will be a multichannel audio artwork at the Chapultepec Mayor lake. Chapultepec is the biggest park in Mexico City, located in the hear of the city and divided into sections. The first section has two lakes of different sizes. The border of the smaller lake is periodically used for dance events. The larger lake has two small islands and has about a hundred plastic boats that visitors can rent. It has an area of 52,852 square meters and a perimeter of 1,079 meters.

The goal of one section of ATL is creating a multichannel sound work at the lake, distributing an amplified central choir, floating drummers, and soundings speakers and metal ensembles. The piece consists also of a set of screens spread over the perimeter of the lake. The piece will have a duration of about 40 minutes and will be repeated for several days during one of the major festivities in the park.

This work will recover the notion of journey that triggered Axial's initial concepts and will also revisit the plurifocality aspect of that work by using a fairly large area for the performance. The compositional structures and sound development will be planned 


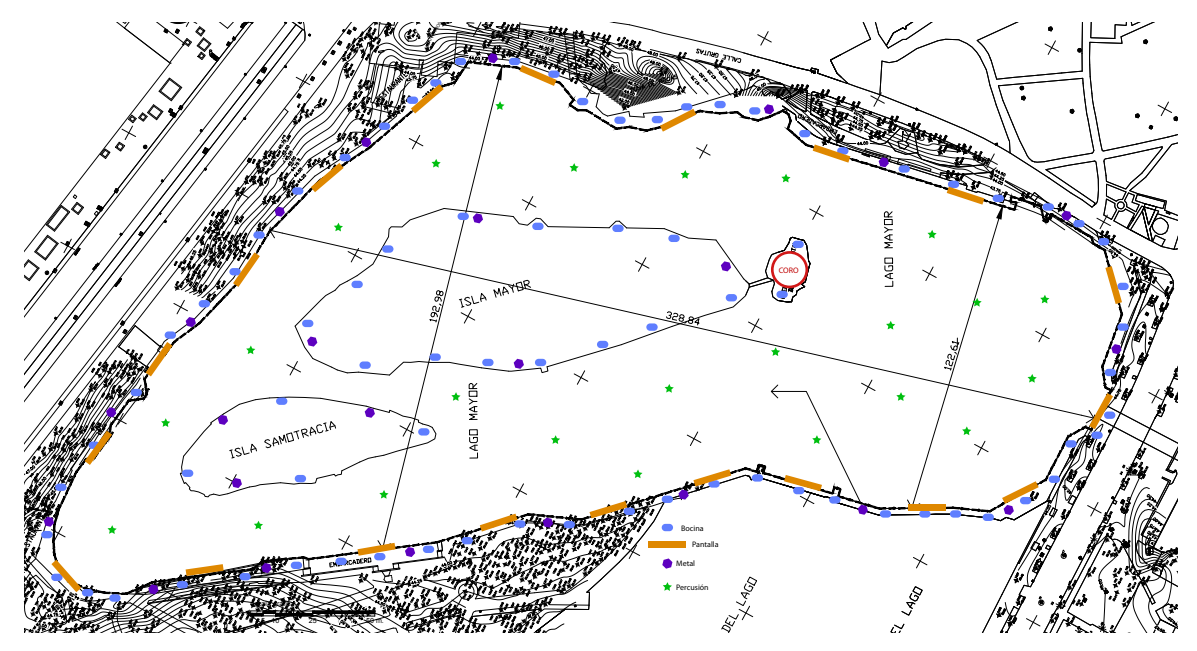

Figure 7.5: Distribution of sonic elements in ATL.

according to the particular space, considering the mobility of the audience and the mobility of some of the sources. Metal assemblies will move the instruments during the performance, affecting sound propagation. Digital sound will be constructed in such a way that sound objects will also move in space. The piece, as with any plurifocal work, should consider the different types of listening that occur in such open and big spaces and also the propagation of sound clouds. 


\section{Appendix A}

\section{DIGITAL MATERIALS}

The following table presents a list and description of all digital materials of personal authorship that constitute the materials, components, and audiovisual documentation for this thesis. This material has been submitted in digital format during the registration process of the thesis, keeping to the submission specifications of the University of Washington. These versions are guaranteed to be preserved and maintained by the University. Additionally, some materials have an external link where identical or alternative versions can be downloaded. The author will try to maintain such documentation, though there is no guarantee as to the long-term persistence of these versions.

\begin{tabular}{|c|c|}
\hline FILE & LINK AND DETAILS \\
\hline \multirow[t]{2}{*}{ juum.zip } & http://hugosolis.net/juum-software/ \\
\hline & Source code of the Juum software. \\
\hline \multirow[t]{2}{*}{ cloudSynthesis.zip } & http://hugosolis.net/cloud-synthesis/ \\
\hline & Source code of the Cloud Synthesis infrastructure. \\
\hline \multirow[t]{2}{*}{ pec.zip } & http://hugosolis.net/plurifocal-events-controller/ \\
\hline & $\begin{array}{l}\text { Source code and Eagle files of the Plurifocal Events Con- } \\
\text { troller. }\end{array}$ \\
\hline \multirow[t]{2}{*}{ axialSound.zip } & http://hugosolis.net/axial/ \\
\hline & Stereo audio mix of the final work. \\
\hline \multirow[t]{2}{*}{ axialVideos.zip } & http://hugosolis.net/axial/ \\
\hline & Two videos of audio recording and one of the installation. \\
\hline
\end{tabular}

Table A.1: List of materials. 


\section{Appendix B \\ PLURIFOCAL EVENTS CONTROLLER SPECIFICATION}

This appendix offers details about the Plurifocal Events Controller. A list of materials with quantity, costs, and number ID is presented. A set of diagrams and schematics, a table of protocol controls, and the core part of the code are also presented.

\section{B.1 Technology of the Plurifocal Events Controller}

The Plurifocal Events Controller uses a set of standard integrated circuits and secondary electronic components such as voltage regulators, capacitors, LEDs, resistors, and clocks. The core of the system is a microcontroller and a ZigBit module. All the components are mounted on a two-layer printed circuit board designed on Eagle and fabricated externally in a professional PCB board factory that uses professional silk screen printing, the main commercial method for high-volume production.

The core elements of the board are two integrated circuits, an AVR ATmega168 and an AVR ZigBit Amp. The former works as the main control, handling the Pulse Wide Modulation (PWM) of all the ports according to the data received by the ZigBit module. The latter handles the wireless connection by establishing communication to the master board at startup and updating live data sent from the computer.

In order to connect both integrates circuits and create a wireless network, both devices had to be programmed using the libraries offered by the fabricant. In the course of this artistic project, several versions were programmed and tested until a robust and efficient version emerged. Details about the creation of the board are presented at Plurifocal Events Controller Specification.

\section{B.1.1 Microcontrollers}

Microcontrollers are integrated circuits that encapsulate a variety of units including a CPU, flash memory, SRAM, analog comparators, analog-to-digital converters, digitalto-analog converters, time generators, EEPROM, USART, Arithmetic Logic Units, and 
general working registers that can be used an input and output ports. With a rich instruction set, these devices can be programmed to execute a variety of digital functions. Due their low cost, small size, and relative power and speed, they are an ideal solution for embedded systems where the sequential logic has been predefined and computer usage is intensive. Their input and output ports make them and ideal solution for creating bridges between the digital world of computers and the analog world familiar to humans.

The term physical computing describes the essence of such dynamics, wherein embedded digital devices interact with real objects such as lights, motors, sensors, knobs, and various gadgets and physical devices. It is natural that the potential of microcontrollers attracts artists interested in interactive experiences where audience feedback and digital generation of material is required. Microcontrollers have to be programmed first in order to set the rules and sequential logic of the device. There is an immense variety of microcontrollers and programming languages but many microcontrollers are based on the C standard. That is the case of the AVR 8-bit microcontrollers. The ATmega168 used on the Plurifocal Events Controller belongs in this category. The ATmega168 was a good option for our module because it is a $20 \mathrm{MHz}$ device with $16 \mathrm{~KB}$ of memory, three timer/counters, six PWM channels, 8-channel 10-bit ADC, USART, and 23 programmable I/O lines. [28]

\section{B.1.2 ZigBee and BitCloud}

Having a wireless connection between devices was important for our artistic proposal because we wanted to control devices distributed over long distances. A cable solution would have been expensive, hard to maintain, and difficult to install and transport. Therefore we studied the wireless options on the market and decided to use the ZigBee technology.

ZigBee is a public standard created in 2003 by a consortium of network companies. For many applications, Wi-Fi and Bluetooth protocols are not good solutions. Wi-Fi requires big infrastructures. Bluetooth was designed for communication over short distances, less than about twenty meters. Neither Wi-Fi nor Bluetooth allows the creation of mesh networks; ZigBee allows such a configuration, where nodes can retransmit messages, allowing the propagation of messages over longer distances. It is a 


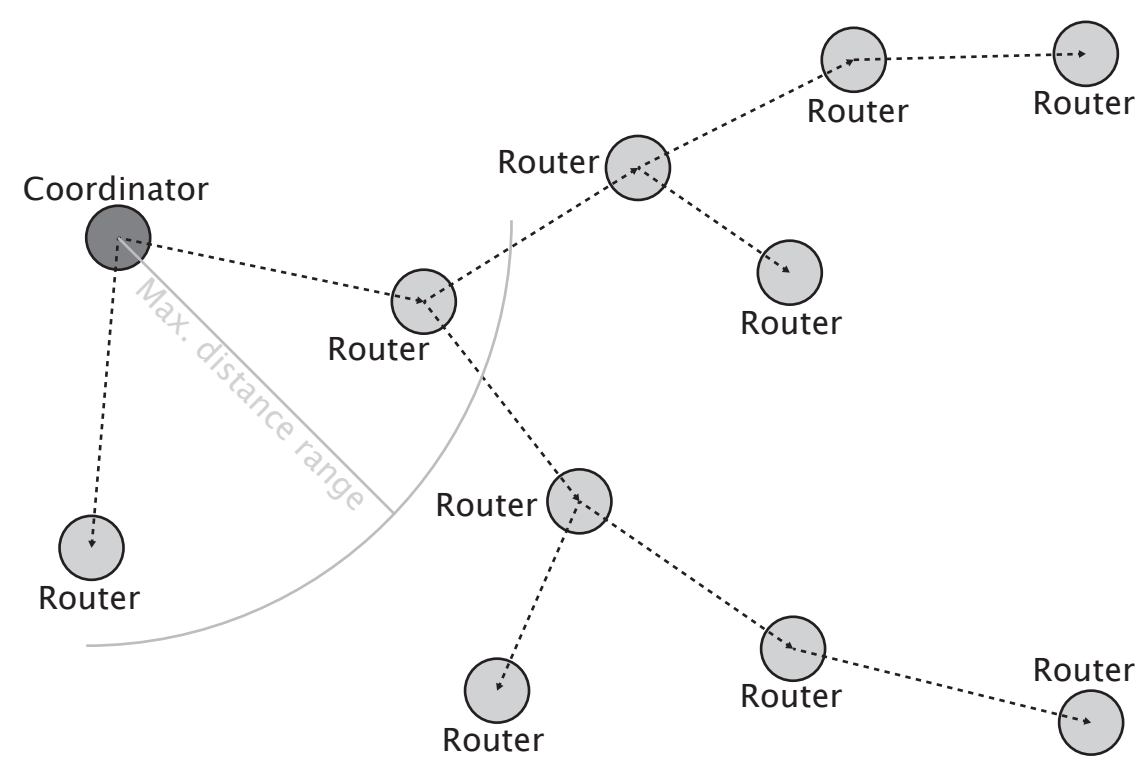

Figure B.1: Mesh topology of a BitCloud network as used by the Plurifocal Events Controller.

low power consumption option, with a bit rate of $250 \mathrm{~KB}$ per second. It is intended for consumer and industrial applications including medical equipment, traffic management systems, home automatization, and other situations that require low data transfer rates over medium-sized distances. The ZigBee network layer supports star, tree, and mesh topologies. In a star topology, the network is controlled by a single coordinator. All the other devices communicate directly with the coordinator. In mesh and tree topologies, the network may be extended with the use of routers. Routers move data and control messages through the network, allowing full peer-to-peer communication.[44]

BitCloud is the commercial name of the API library built on top of the ZigBee protocol by Atmel Corporation. BitCloud can be used on all of the ZigBit devices from this company including the ZigBit (ATZB-DK-24), ZigBit Amp (ATZB-DK-A24), and ZigBit 900 (ATZB-DK-900). BitCloud is a set of C functions that allows users to create entire ZigBit applications without starting from scratch. In addition, it has a top layer called SerialNet that allows users to call several of the $\mathrm{C}$ functions with a light serial protocol. SerialNet was used on the fist development versions of our module. 
[1][34][2][45] BitCloud in C was used over the last versions in order to have better control of the communication and mesh networks.

\section{B.2 Fabrication of Electronics}

Creating the Plurifocal Events Controller was an iterative project of several months. Several versions were created over time, and adaptation and errors that occurred during the evolution of the idea helped to improve the work. As mentioned earlier, the circuit was designed with Eagle software, which allows the generation of electronic schematics. From the schematics a trace diagram is created. The diagram can be printed for creating hobby boards using chemical etching techniques or can be saved in the Gerber format. This format is used for CNC milling machines or for industrial fabrication.

In our case, we produced a prototype with a CNC machine and later fabricated boards with professional silk screen printing. For all the produced versions, the electronic components were mounted by hand and soldered one by one with the help of magnifying glasses.

\section{B.2.1 Prototype}

In order to test our technology, the first step was the creation of a testing prototype. The two-layer diagram was created with Eagle and then printed with the Circuit Board Plotter at the university. A significant amount of time was employed joining both sides of the thru-holes by hand. Most of the components were thru-hole devices, increasing the size and complicating the layering. While the prototype worked fine and helped for testing purposes, it was clear this would not be the technology for mass production.

\section{B.2.2 First Version}

Once the prototype worked fine and we were able to communicate wirelessly between the board and a AVR Raven board, we proceeded to fabricate the first version of the board with the specific goal of controlling as many low-power motors as possible. The board is simple, with a ZigBit module, a Mega 168, and a set of twelve TPIC6A596DW 8-output shift registers with power transistors integrated on each output. Having the power transistor inside the shift register simplified the layout significantly and we managed to 


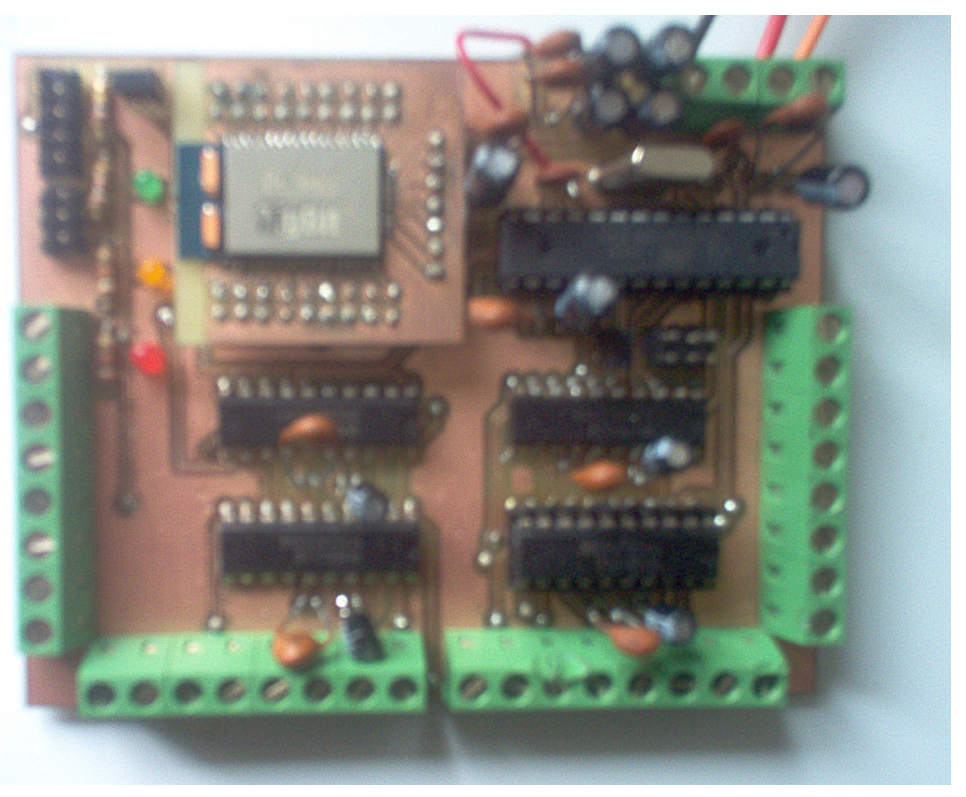

Figure B.2: First prototype of the Plurifocal Events Controller.

have 96 outputs that could control small motors without too much external equipment. This board also has eight ports that worked as analog inputs, allowing the sensing of external changes.

\section{Code and Protocol}

Two different but interconnected pieces of code had to be developed for the module: the code that handles the ZigBee communication inside the ZigBit module, and the code in the main microcontroller that handles the motor controls and communicates with the ZigBee module.

With the physical board fabricated, several code versions were tested. Initially, the ZigBee module used the SerialNet prefabricated solution. It allowed connecting to the network, sending, and receiving messages easily but some parameters could not be selected and it worked erratically under certain circumstances. Later, the SerialNet version was replaced by custom software that allowed star network configurations. At that point there were no artistic projects that required mesh networks.

The code of the main microcontroller also changed several times, not only with 


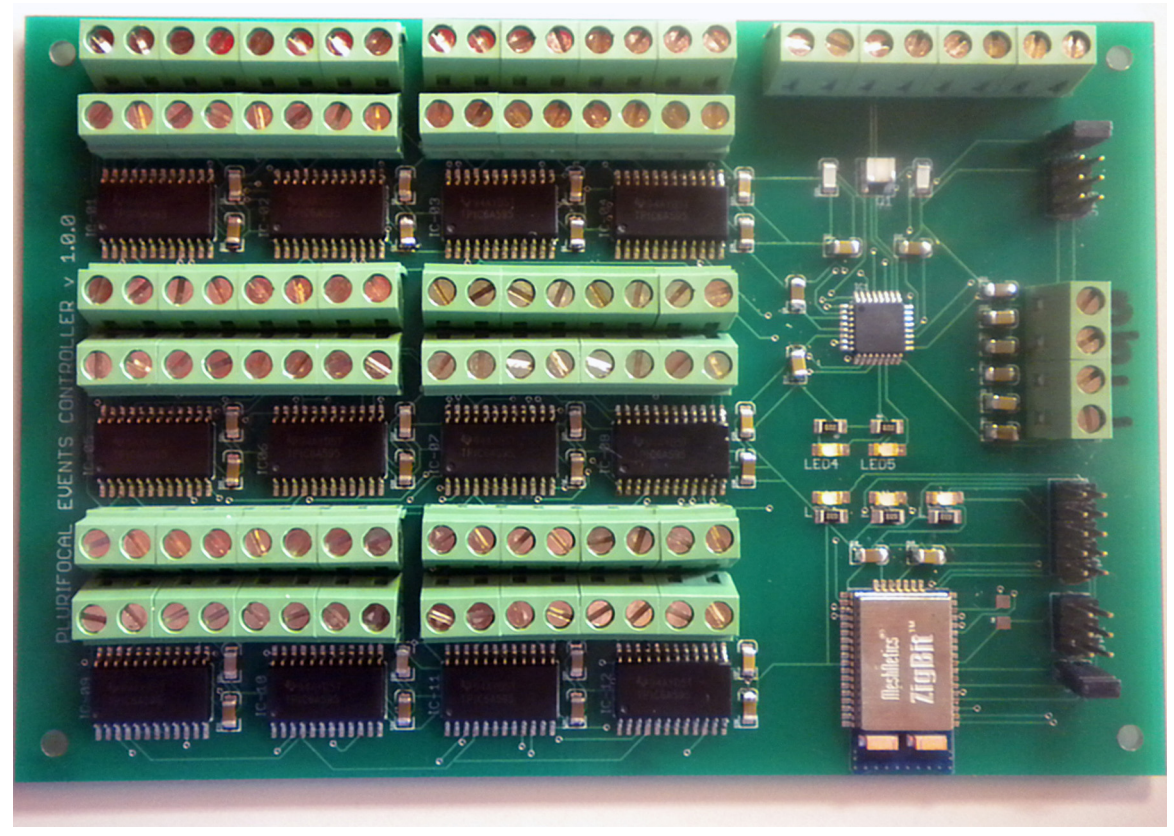

Figure B.3: First version of the Plurifocal Events Controller.

changes in the code of the ZigBit module, but also with the exploration of the sonic properties of the motors. While the parameters, ranges, and protocols changed over the time, the logic or state flow remains similar. At boot loading, the code asks the ZigBit module to connect to the network and waits until the connection has stablished. Once the board is connected to the network, it starts sending frequent messages to the coordinator with the values of the eight ADC registers. It also activates an interrupt that waits for messages coming from the ZigBit module. The message that comes from the coordinator represents the relative value that each output port should set. The board handles only discrete on/off values for the motors. The code and the protocol are presented in Plurifocal Events Controller Specification.

\section{B.2.3 Second Version}

The first version of the board was used on all of the series of Dead Pianos art pieces, and worked without any problems; however, while planning new works, it became clear that two important elements needed to be updated. The first one was the desire to 


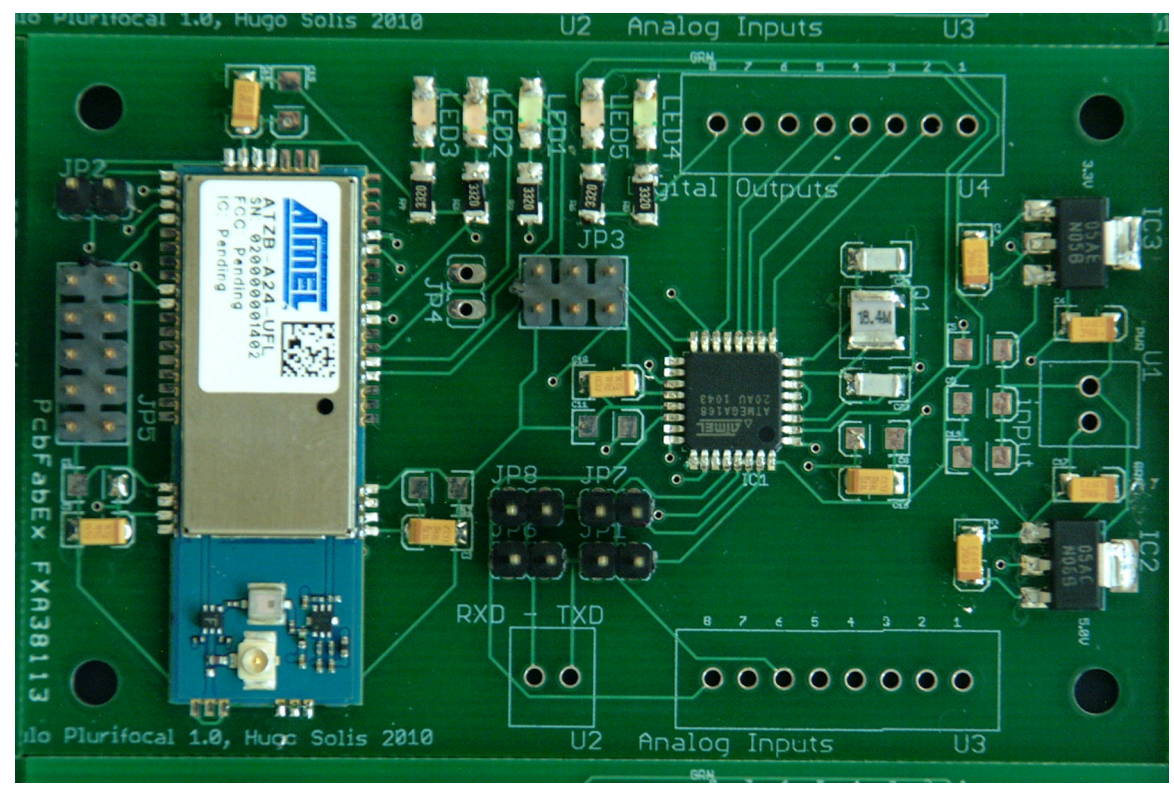

Figure B.4: Second version of the Plurifocal Events Controller (board in process).

spread the percussion devices over bigger spaces which meant we needed to use bigger antennas; the second was the possibility of controlling bigger motors which meant we needed bigger power amplifiers.

With this in mind, a second version of the Plurifocal Events Controller was designed and produced. With the experience of the previous board, a smaller modular circuit with better configuration was created. The board now included a set of voltage regulators, which allows us to use a variety of batteries or external regulators. The board now uses the ZigBit amp module with external antenna, offering a much longer connection of about 100 meters.

Since the goal of this board was to control a few devices over longer distances rather than controlling many motors near each other, it has only eight output ports. On the other hand, the benefit is that with two internal clocks and an arrays of variables, the output of each port is now adjustable using Pulse Wide Modulation (PWM). Using PWM on the outputs allowed us to experiment with the force and speed of the solenoids and motors, allowing for the generation of dynamics and changes of amplitude on the sonic materials. 


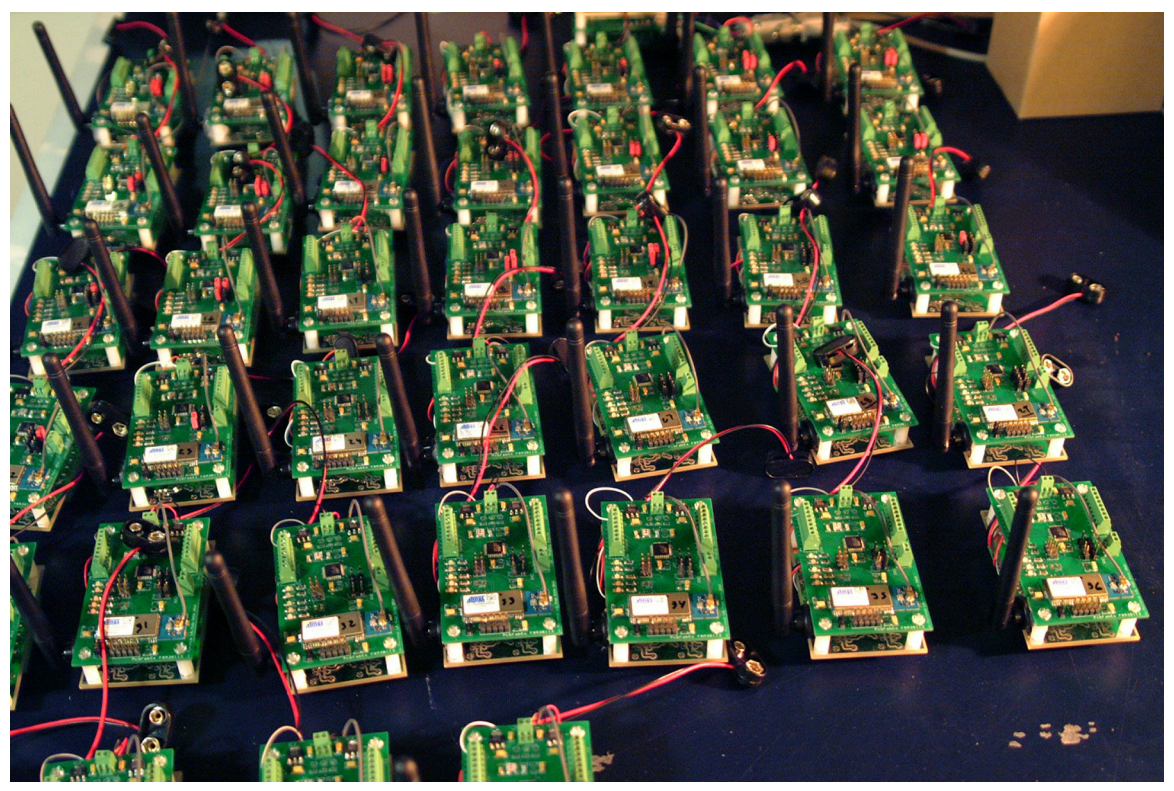

Figure B.5: Second version of the Plurifocal Events Controller finished.

The board do not have any power amplifier, leaving the power capabilities to a secondary board that can be modified or substituted according to the artwork. To date, only one version of the power board has been developed. Using power Darlington transistors and "bare bones" PCBs, a cheap solution of four single-direction and one bidirectional power amplifiers were cheaply fabricated. The TIP transistors allow the control of medium-sized, high-current motors and solenoids.

\section{Code and Protocol}

As in the case of the first board, several versions of the code were developed over time, the last version proving to be a robust and malleable solution. The ZigBit module contains a custom $\mathrm{C}$ code that connects automatically to the mesh network, setting each board as a router. It has a set of simple but important features; for example, if the connection is lost the module tries to reconnect, if corrupted messages are received they are discarded, etc.

The code at the main microcontroller had the most significant improvement. Leaving all the connection and radio functionality to the ZigBit module permits the ATmega168 
to focus on the control of solenoids and motors. With a direct connection between the custom register of the microcontroller and the output pins, a better resolution was gained. The control speed of the ports is now fast enough to control not only the speed and force but also the pulse of repetition. The protocol allows individual addressing, synchronization, global parameters, and change of modulation in such a way that the data transmission over the air is reduced in comparison to the previous iterations. The simplification of the messages and the reduction of transmitted data produced a highly reliable network with better resolution, allowing many more types of sound generation and rhythmic patterns.

\section{B.3 Fabrication of Mechanical Components}

The rationale for developing the Plurifocal Events Controller system was to produce acoustic sounds controlled with digital resolution spread over large areas. However, at the end of the process, the sound has to be generated with some kind of mechanical activator. Over the years there was a secondary exploration of motors and solenoids. The exploration was certainly not methodological and it was based more on pragmatic and economic concerns.

Acquiring new motors and solenoids in large quantities can be extremely expensive. For this reason obtaining used parts or getting them at surplus stores was a natural $\operatorname{path}^{1}$.

The first explorations were made with small DC motors that allowed us to rub piano strings. While the sound was attractive, the lifespan was extremely short. Later medium-sized DC motors were used as pendular hitters. Still later, extremely small vibrating motors were used to vibrate piano strings by empathy. The exploration of using motors to hit objects in a semi-chaotic but rhythmic manner continued during an art residency in Japan. Back in Mexico, a set of powerful solenoids was finally obtained and a percussion mechanism was developed which allowed strong and precise attacks.

\footnotetext{
${ }^{1}$ The online store http://allelectronics.com was the main source for motors and solenoids in the USA. In Japan, the Akihabara district was an extraordinary place to get some motors. In Mexico the street of República del Salvador in Downtown is also a good place to get used motors and solenoids from printers and recycled electronic equipment.
} 


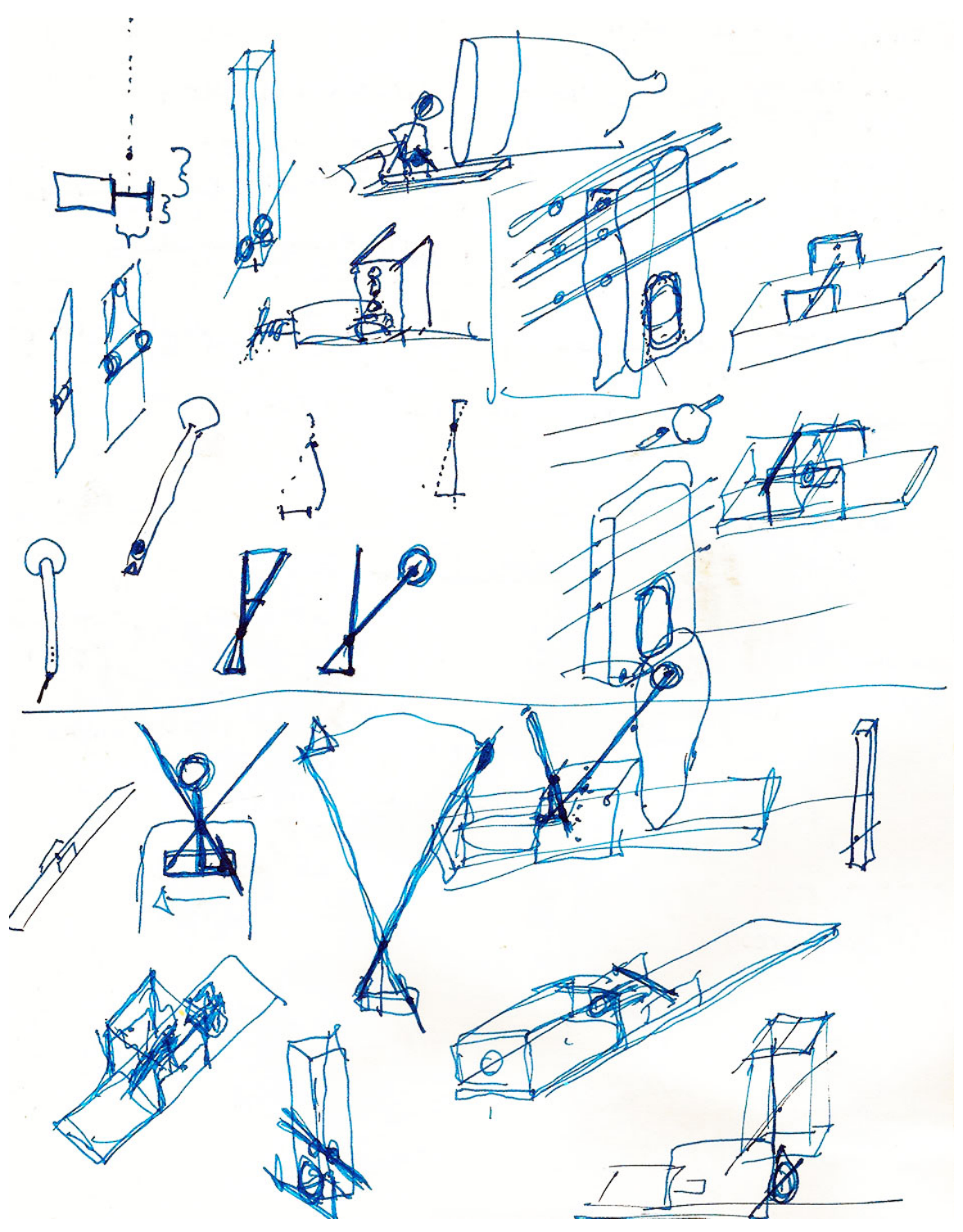

Figure B.6: Initial sketches for solenoid mechanism. 


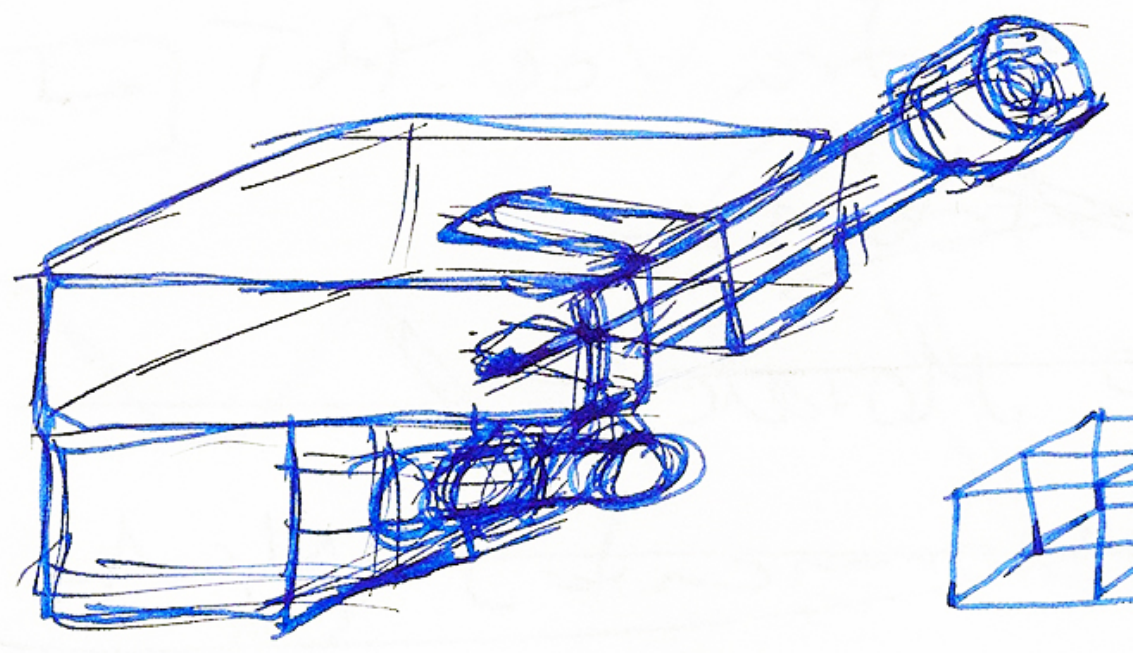

Figure B.7: Sketch of solenoid mechanism.

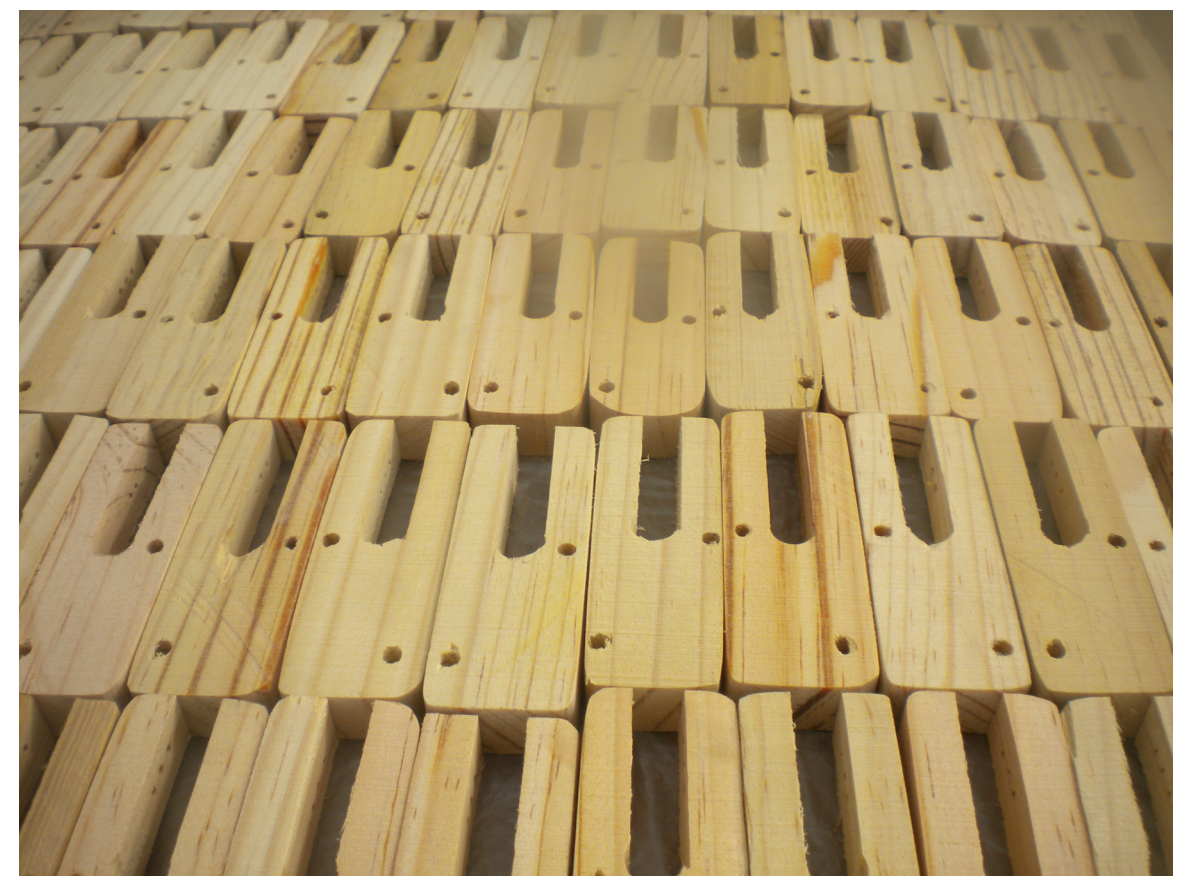

Figure B.8: Wood bases for solenoids. 


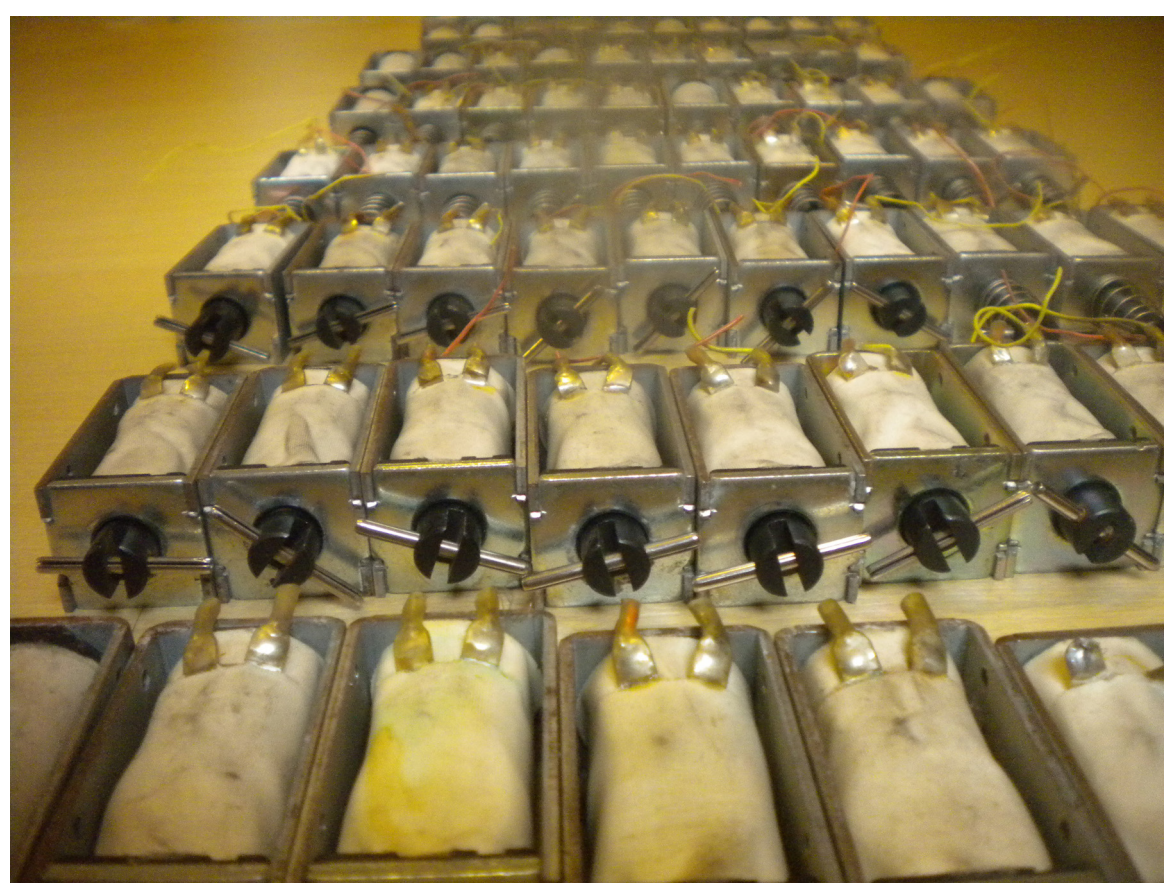

Figure B.9: Twenty-four volt solenoids.

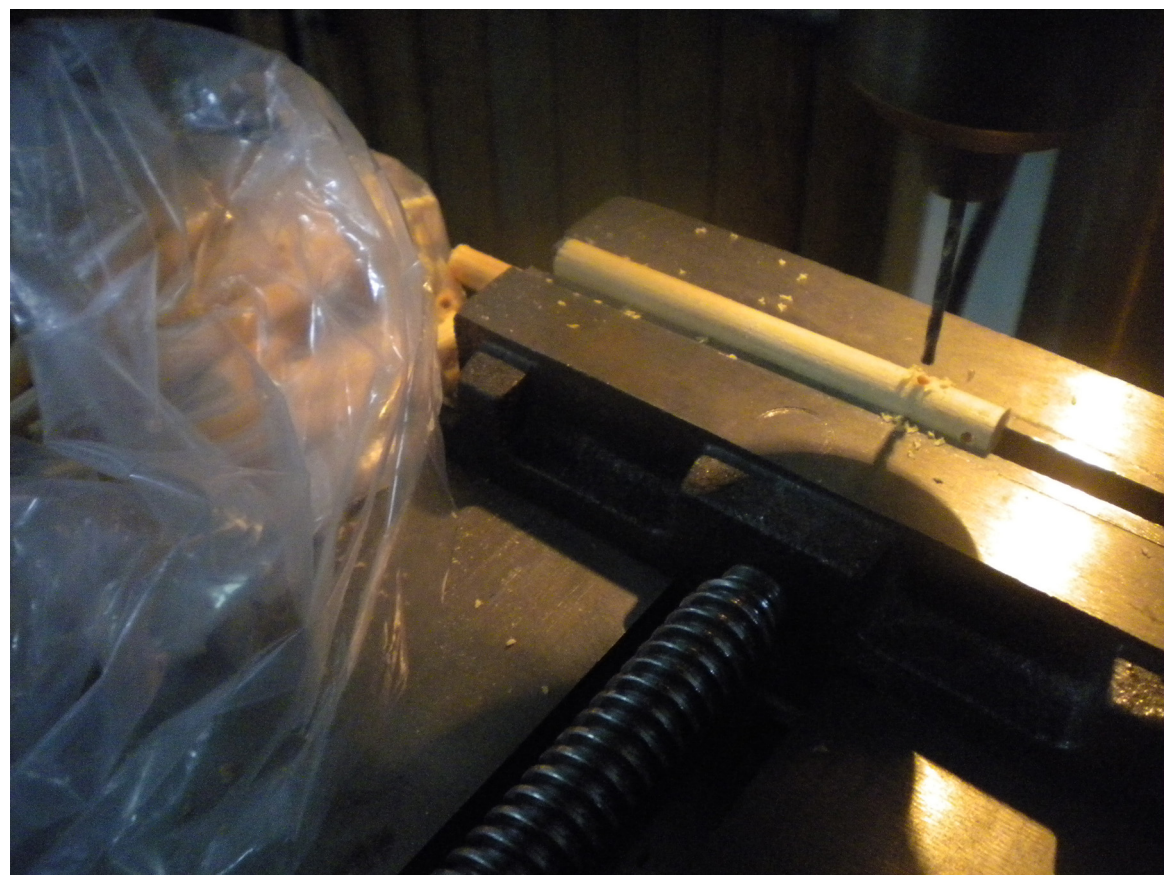

Figure B.10: Drilling sticks for percussion. 


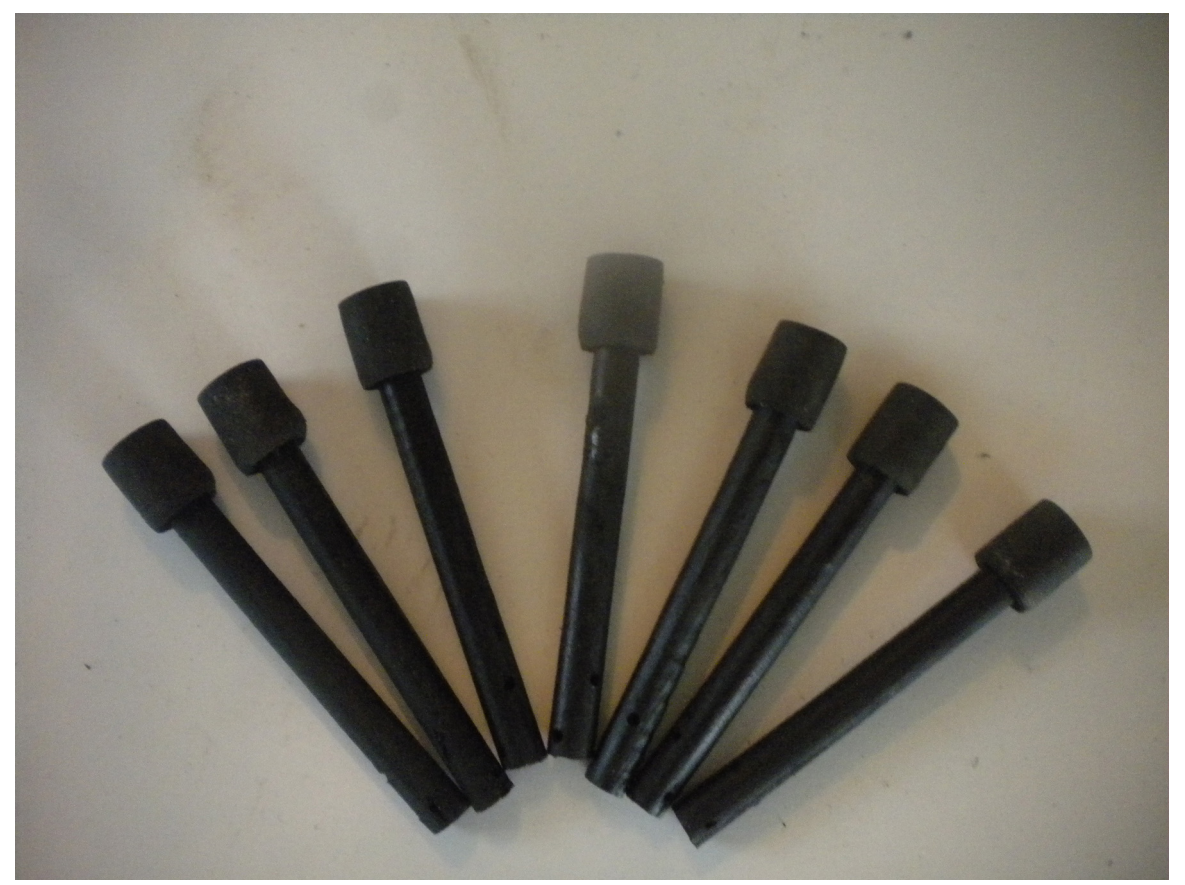

Figure B.11: Damper heads for hitting sticks.

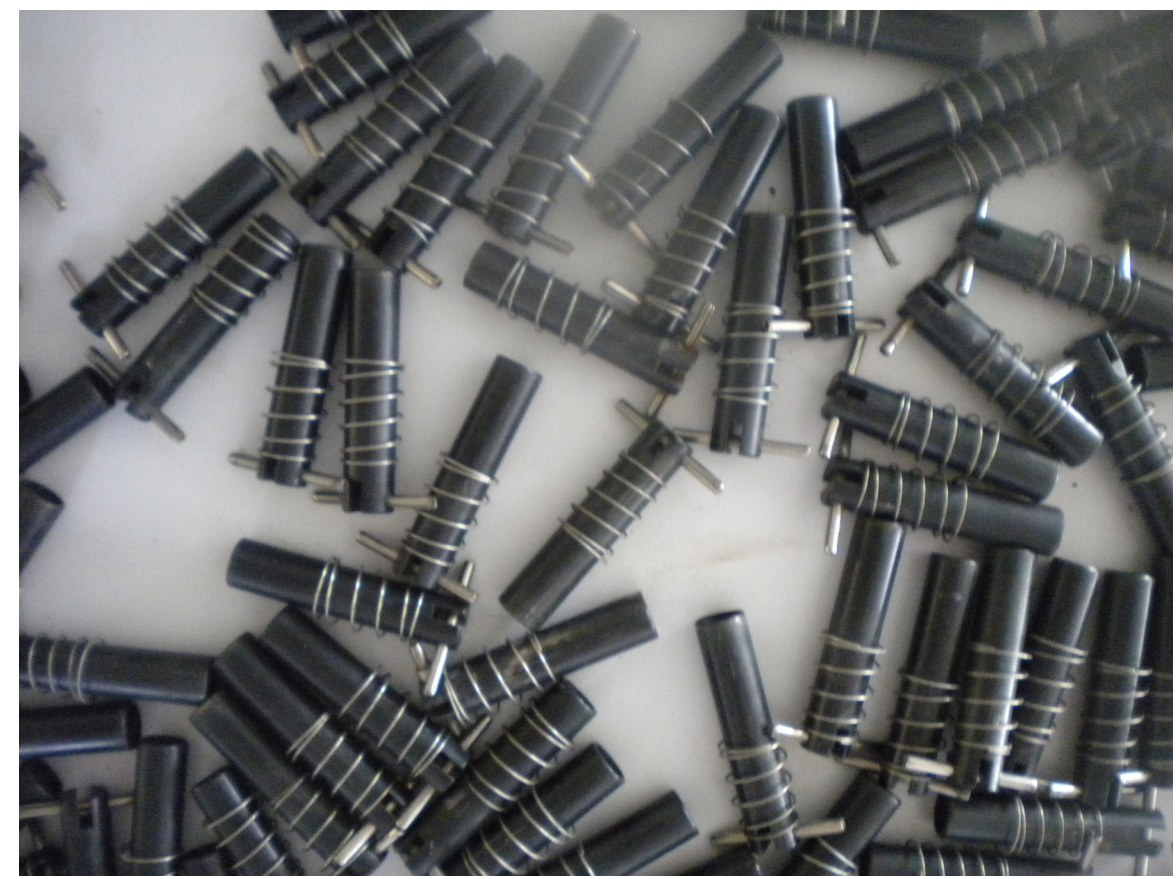

Figure B.12: Adjusting and placement of springs into the solenoids. 


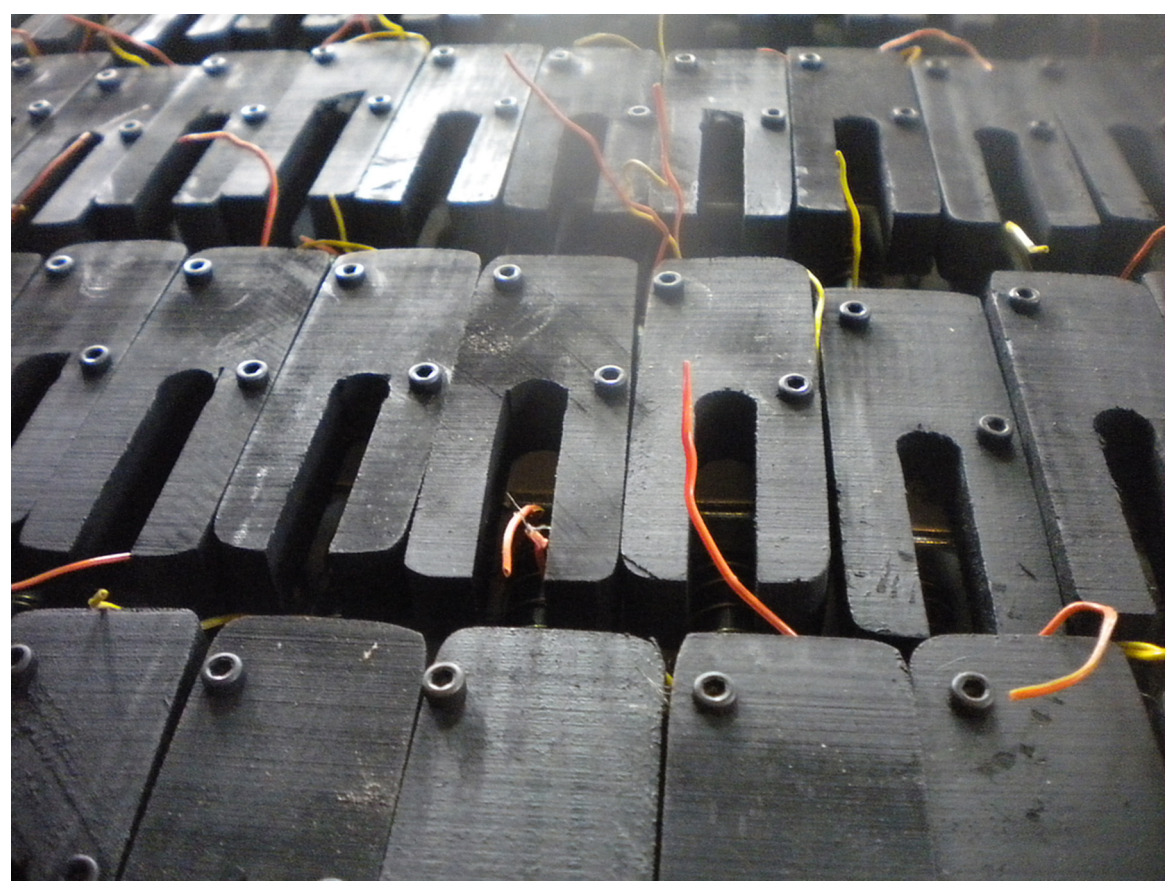

Figure B.13: Assembly of bases and solenoids.

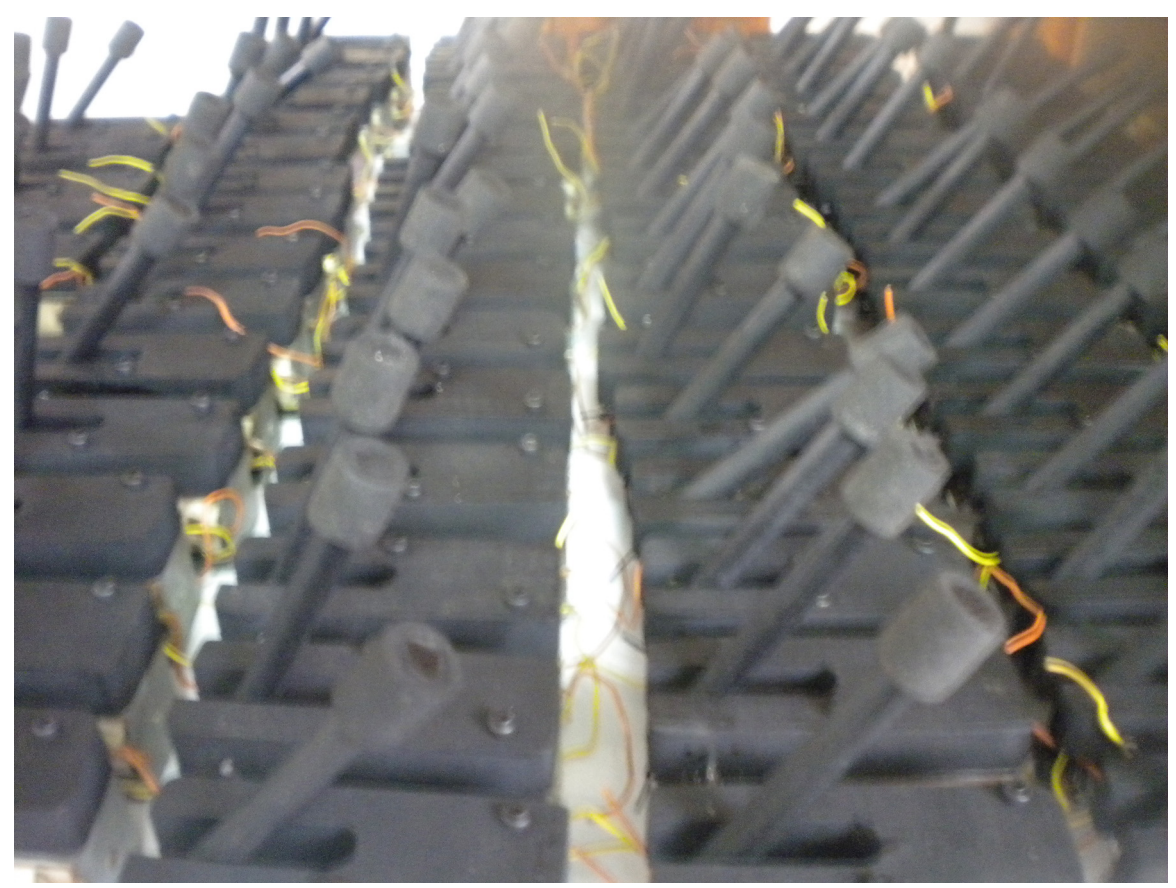

Figure B.14: Set of solenoids and sticks finished. 


\section{B.4 Applications of the Plurifocal Events Controller}

The different iterations and versions of the Plurifocal Events Controller have been used in several artworks. The details of these works can be found on the artists webpage. However, it is important to list the works that have been created with the modules specially because of the creative feedback loop between the artistic and expressive goals of each work and the development of the module.

Metaphors for Dead Pianos I. The first version of the module was employed in this work, in which several DC motors were controlled by the board. Using the SerialNet protocol, a small microcomputer sent messages to the board. For this first work there were no modulation values so only on or off were possible. Since the DC motors consumed about 800 milliamperes each, an external power board with an array of power transistor was needed in addition to the integrated transistors.

Metaphors for Dead Pianos II. The second version of this work, which also used the first version of the board, required four suspended pianos. The SerialNet still in use, now with a star network configuration. The boards were also controlled by a small Linux microcomputer. Motors were hung from cables bouncing chaotically on the sound board. These new motors did not consume much current, therefore no external power board was used.

Metaphors for Dead Pianos III. The third version of this work used two pianos and also employed the first version of the board. A custom BitCloud Application was used in the ZigBit modules, and the communication protocol was updated. Tiny vibrator motors employed glue and silicon, simplifying the layout of the work and offering a much better pallet of sounds. The microcomputer was replaced by a regular computer and the coordinator board was connected by USB.

Ensemble Modern Concert. The second version of the Plurifocal Events Controller premiered with this performance, in which twelve of the boards were distributed over an entire concert hall. Each board activated motors that struck the metallic 


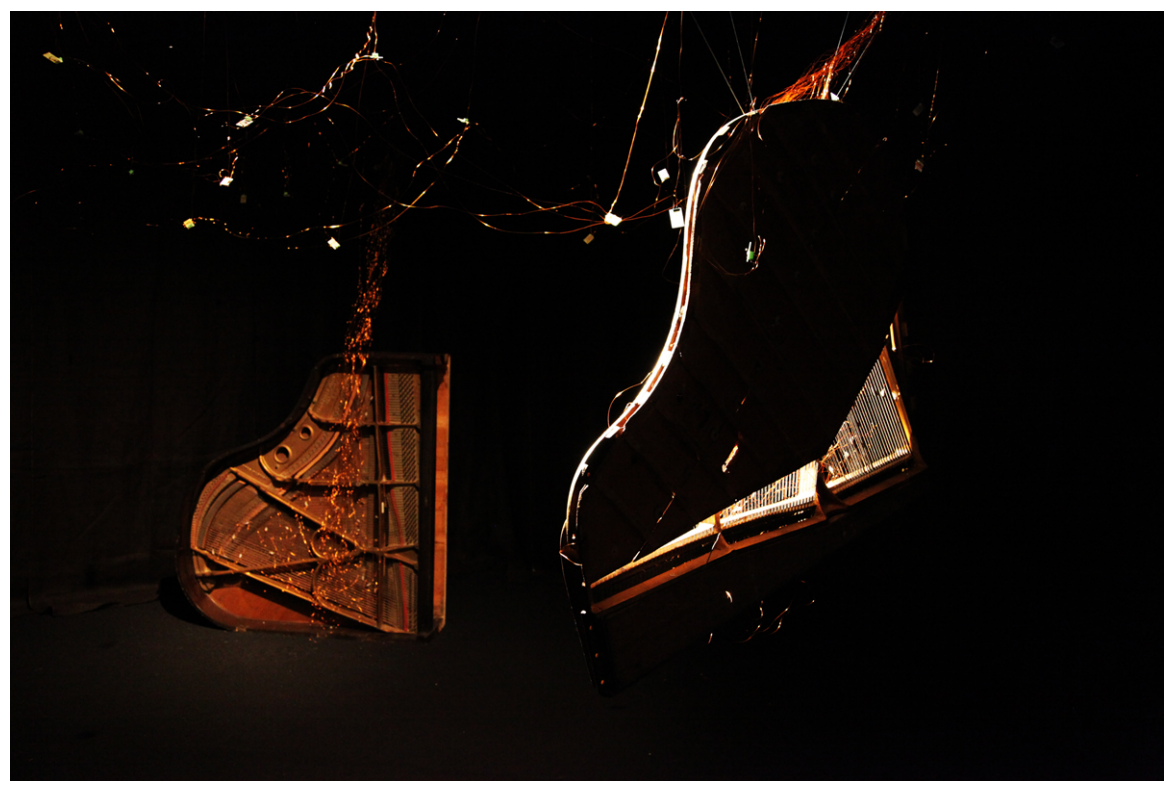

Figure B.15: Metaphors for Dead Pianos at the Jack Straw Gallery.

walls of the venue. SerialNet was used again, but PWM on the ports was now possible and the use of a mesh topology allowed a connection over longer distances.

Concert for piano, viola, and gallery. Also using the new version of the board, the walls of the gallery were struck in a similar fashion to the previous work. Minor changes to the protocols and code were tested.

Resonant Objects. The second version of the board was used for controlling 96 highpowered solenoids. The transmit protocol was updated, synchronization parameters were developed, but we returned to the unmodulated on/off condition.

Echoes for Forgotten Locations. This piece employed a physical configuration similar to the previous work but with updated software that allowed a wide variety of sonic textures using PWM.

Axial. For this work, all the previous experience contributed to the achievement of a robust and efficient technology. The data protocol allowed the modulation not 


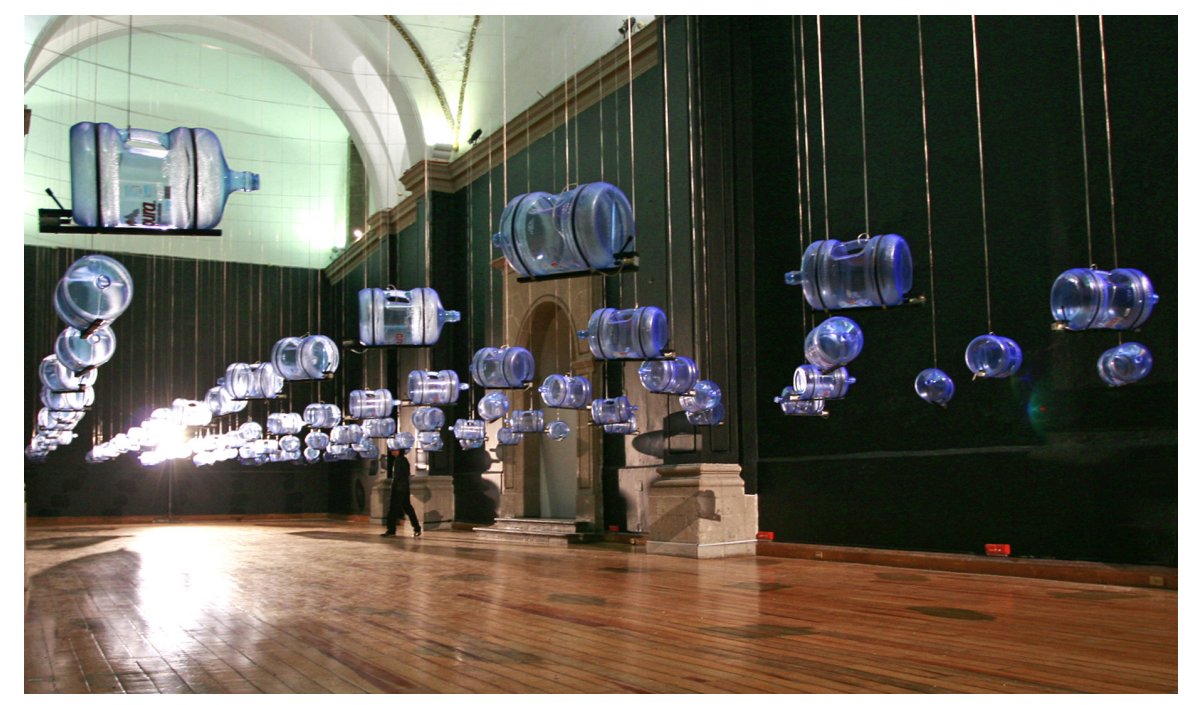

Figure B.16: Objetos Resonantes at the Laboratorio Arte Alameda in México City. (Photo: Sergio Rosas)

only of volume but also of rhythmic structures. A collection of messages for synchronization and global changes allowed rhythmic games. The rhythmic pulses were now generated inside the board if needed, reducing significantly the transmitted data between modules, which are now in a mesh network of routers allowing connections of several hundred meters. A custom BitCloud code replaced the SerialNet, creating a much more robust wireless network.

\section{B.5 Materials}

Table B.1 lists the parts required for building one module. Table B.2 lists the parts required for building the power module ${ }^{2}$.

\begin{tabular}{|c|c|c|c|}
\hline Quantity & description & Digi-key part number & Unit price $(\mathrm{Dls})$ \\
\hline 01 & $\begin{array}{l}\text { IC AVR MCU } 16 \mathrm{~K} \\
20 \mathrm{MHZ} 32 \mathrm{TQFP}\end{array}$ & ATMEGA168-20AU-ND & $\$ 2.71$ \\
\hline
\end{tabular}

\footnotetext{
${ }^{2}$ It is not recommended to replicate the power module since there are simpler and more economical modules to drive motors and solenoids.
} 


\begin{tabular}{|c|c|c|c|}
\hline 01 & ZigBit Module & ATZB-A24-UFLRDKR-ND & $\$ 30.70$ \\
\hline 01 & $\begin{array}{l}\text { RESONATOR CER } \\
\text { 18.43MHZ SMD }\end{array}$ & 535-10026-1-ND & $\$ 0.34$ \\
\hline 02 & $\begin{array}{l}\text { LED RED FACE UP } \\
1206\end{array}$ & P11141CT-ND & $\$ 0.28$ \\
\hline 01 & $\begin{array}{l}\text { LED ORANGE FACE } \\
\text { UP } 1206\end{array}$ & P11526CT-ND & $\$ 0.32$ \\
\hline 02 & $\begin{array}{l}\text { LED GREEN FACE } \\
\text { UP } 1206\end{array}$ & P11529CT-ND & $\$ 0.29$ \\
\hline 09 & $\begin{array}{l}\text { CAP CERAMIC } 22 \mathrm{PF} \\
50 \mathrm{~V} \text { NP0 } 1206\end{array}$ & 311-1154-1-ND & $\$ 0.07$ \\
\hline 09 & $\begin{array}{l}\text { CAP CER 1.0UF 16V } \\
10 \% \text { X7R } 1206\end{array}$ & 399-3678-1-ND & $\$ 0.13$ \\
\hline 09 & $\begin{array}{l}\text { CAP CER 10UF } 16 \mathrm{~V} \\
\text { X7R } 1206\end{array}$ & $478-1655-1-\mathrm{ND}$ & $\$ 0.35$ \\
\hline 01 & $\begin{array}{l}\text { CAP CER 100UF 6.3V } \\
\text { X5R } 1206\end{array}$ & 718-1740-1-ND & $\$ 0.76$ \\
\hline 05 & $\begin{array}{l}\text { RES } 332 \text { OHM } 1 / 8 \mathrm{~W} \\
.1 \% 1206 \mathrm{SMD}\end{array}$ & RNCF32T2332BICT-ND & $\$ 0.20$ \\
\hline 02 & $\begin{array}{l}\text { TERM BLOCK } 2 \mathrm{POS} \\
\text { SIDE ENT } 2.54 \mathrm{MM}\end{array}$ & A98333-ND & $\$ 0.53$ \\
\hline 02 & $\begin{array}{l}\text { TERM BLOCK } 8 \text { POS } \\
\text { SIDE ENT } 2.54 \mathrm{MM}\end{array}$ & A98338-ND & $\$ 2.16$ \\
\hline 01 & $\begin{array}{l}\text { IC REG LDO } 800 \mathrm{MA} \\
5.0 \mathrm{~V} \text { SOT-223 }\end{array}$ & LM1117IMP-5.0CT-ND & $\$ 1.17$ \\
\hline 01 & $\begin{array}{l}\text { IC REG LDO } 800 \mathrm{MA} \\
3.3 \mathrm{~V} \text { SOT-223 }\end{array}$ & LM1117IMP-3.3CT-ND & $\$ 1.17$ \\
\hline 01 & $\begin{array}{ll}\text { ANTENNA } & \text { RUBB } \\
\text { DUCK } 2.4 \mathrm{GHZ} & 6 \text { "CA- } \\
\text { BLE } & \end{array}$ & 730-1004-ND & $\$ 13.84$ \\
\hline
\end{tabular}




\begin{tabular}{l|lr|r|r}
02 & CONN & HEADER & 609-3363-ND & $\$ 0.25$ \\
& 12POS .100 STR 30AU
\end{tabular} \mid

Table B.1: Plurifocal Events Controller list of components.

\section{B.6 Diagrams}

The following four graphics show the schematics and diagrams of the final version of the Plurifocal Events Controller and the power module. Both boards were designed using the freeware version of the Eagle software (http://www.cadsoftusa.com). Both boards are $4 \times 6$ inches. Wiring of each board required several hours of trial and error in order to generate the optimal two-layer board routes. The boards were manufactured by the PC Fab Express company (http://www.pcbfabexpress.com). Digital files are included in Digital Materials. 


\begin{tabular}{|c|c|c|c|}
\hline Quantity & description & Digi-key part number & Unit price (Dls) \\
\hline 08 & $\begin{array}{l}\text { TRANS NPN DARL } \\
60 \mathrm{~V} 5 \mathrm{~A} \text { TO-220 }\end{array}$ & TIP120-ND & $\$ 0.37$ \\
\hline 08 & $\begin{array}{l}\text { DIODE GEN PUR- } \\
\text { POSE 50V 1A DO41 }\end{array}$ & 1N4001FSCT-ND & $\$ 0.06$ \\
\hline 08 & Resistance & NA & $\$ N A$ \\
\hline 01 & $\begin{array}{l}\text { TERM BLOCK } 2 \mathrm{POS} \\
\text { SIDE ENT } 2.54 \mathrm{MM}\end{array}$ & A98333-ND & $\$ 0.53$ \\
\hline 01 & $\begin{array}{l}\text { TERM BLOCK } 6 \text { POS } \\
\text { SIDE ENT } 2.54 \mathrm{MM}\end{array}$ & A98337-ND & $\$ 1.76$ \\
\hline 01 & $\begin{array}{l}\text { TERM BLOCK } 2 \mathrm{POS} \\
\text { SIDE ENT } 5.0 \mathrm{MM}\end{array}$ & A98076-ND & $\$ 0.39$ \\
\hline 01 & $\begin{array}{l}\text { TERM BLOCK } 8 \text { POS } \\
\text { SIDE ENT } 5.0 \mathrm{MM}\end{array}$ & A98080-ND & $\$ 1.45$ \\
\hline
\end{tabular}

Table B.2: Plurifocal Events Controller list of power module components. 


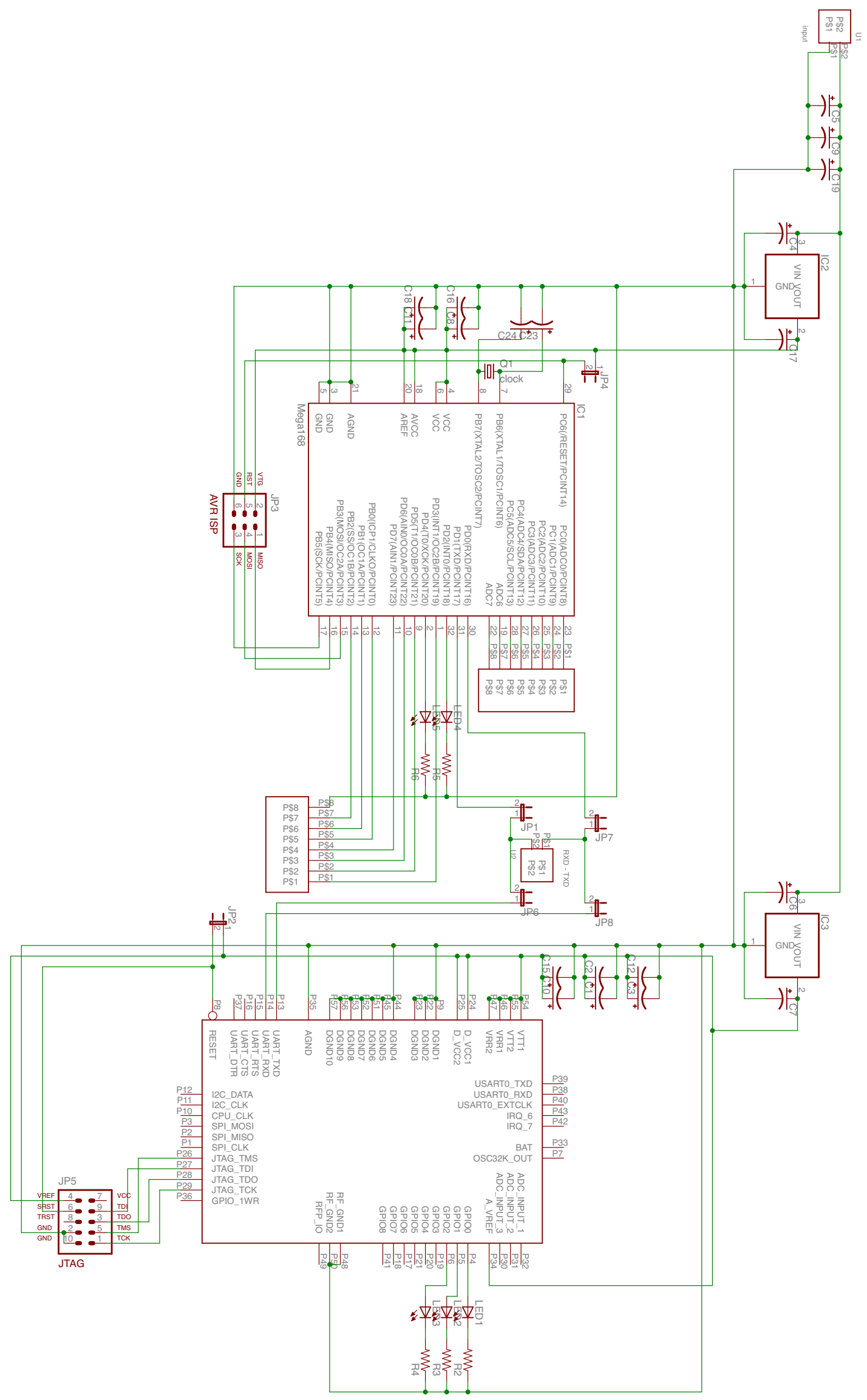




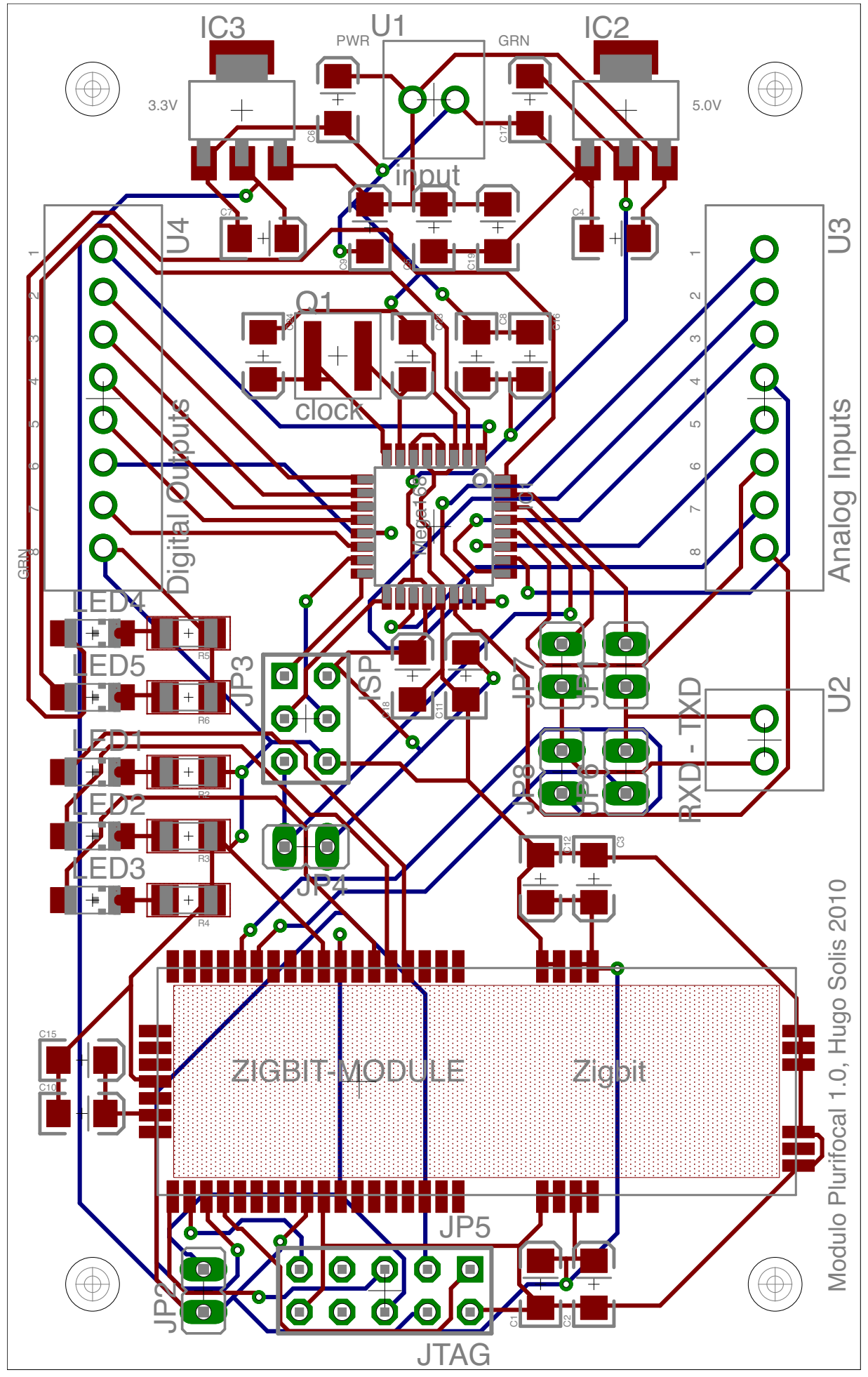




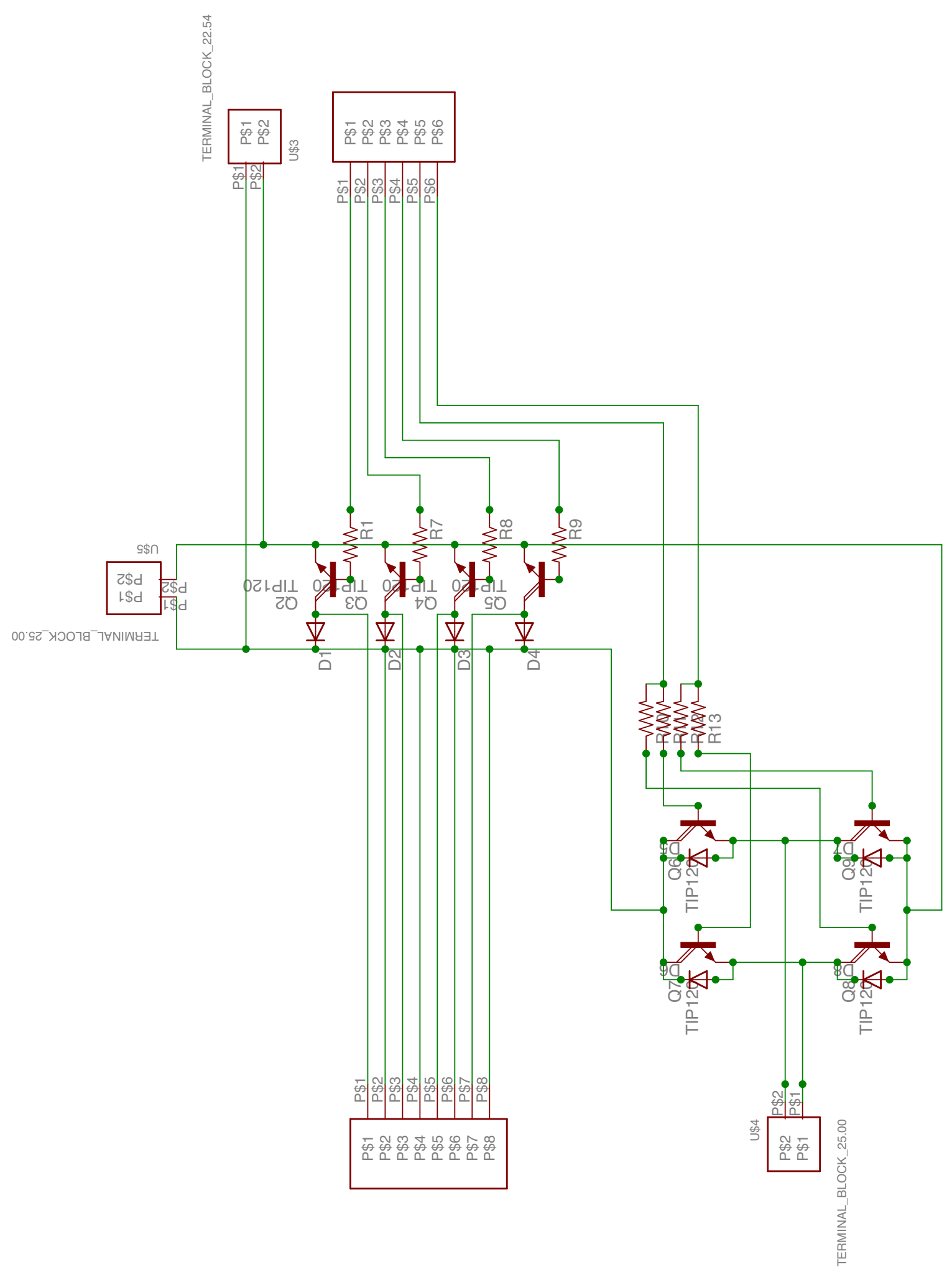




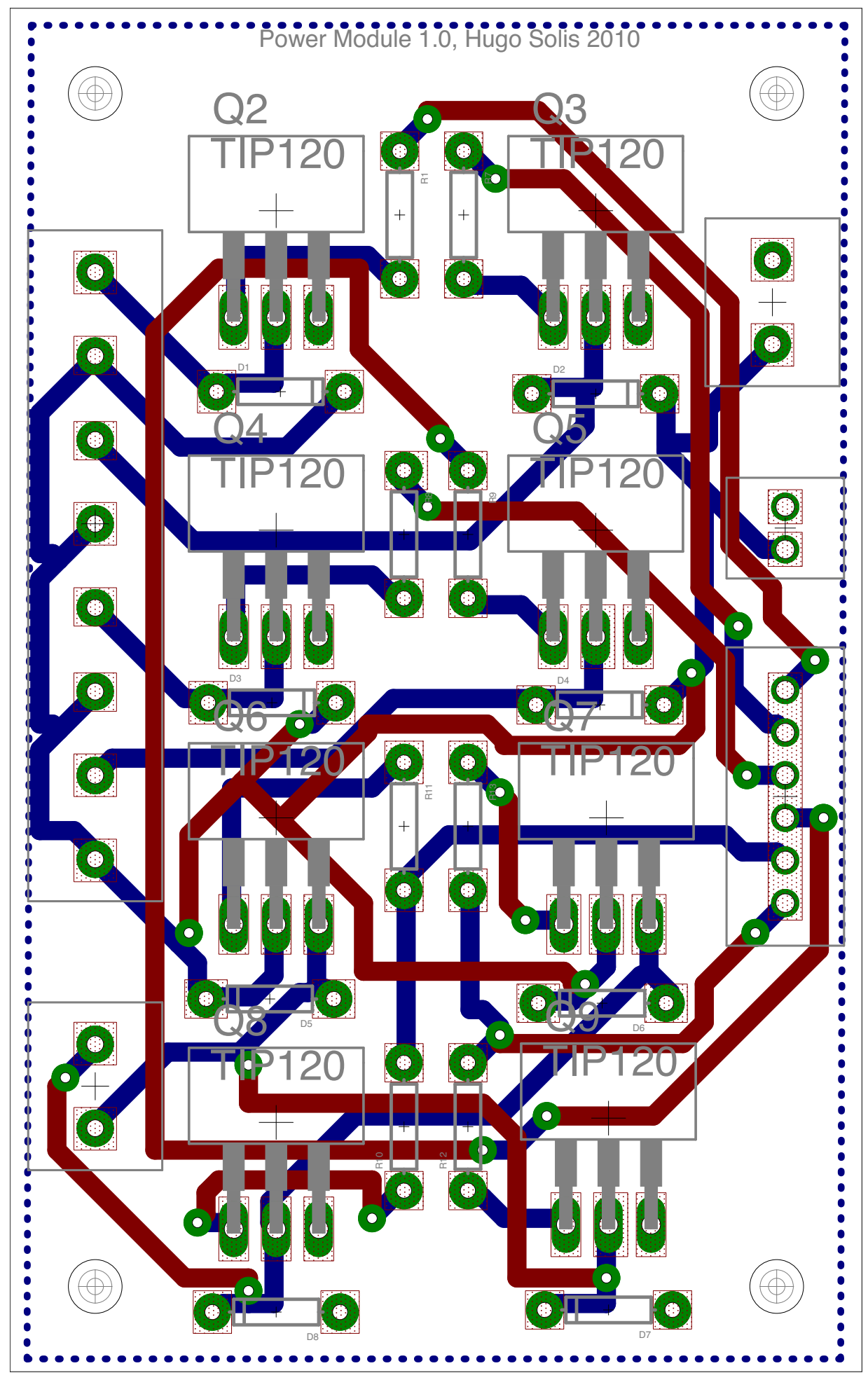




\section{B.7 Protocol}

\section{B.7.1 ZigBee Protocol}

The ZigBee protocol loaded in the ZigBee module is a small variation of the Peer2Peer code included as an example of the API for the BitCloud package provided by ATMEL. While the original Peer2Peer example provides a serial communication between a master device and a slave device, the modification allows the propagation of messages among all of the slave devices and then the extraction of the target ID as a filtering mechanism. This modification allows one master device to send messages to addressed slaves and also to receive messages from all the slaves. The source code is included in the file pec.zip in Digital Materials.

\section{B.7.2 Rhythm Protocol}

The data transmitted over the wireless connection is small, allowing a fast and secure data transmission. Messages are transited line by line, formatted as string messaged. Each section of the message is separated by a blank space. The full source code is included in Digital Materials.

Tables B.3 and B.4 presents the data protocol. Table B.5 presents some examples for guidance.

\section{B.8 Code}

The following code presents the instructions loaded into the ATmega168 microcontroller by the Plurifocal modules. This code was created as a project in the AVR Studio IDE. It can be observed that the code is reading serial data using an interrupt. Once the message is complete, it is interpreted by the main routine which uses it to modify two different Pulse Wide Modulation (PWM) processes. One PWM is used to control the intensity and the other the rhythmic pulse. In order to have these two PWMs, two timers have to be set up at the init function. The main function interprets the serial data using the protocol described at Rhythm Protocol. The source code of the $\mathrm{C}$ file and the $\mathrm{H}$ file are included in the file pec.zip of Digital Materials. 
\#include <avr/io.h>

\#include <avr/interrupt.h>

\#include <inttypes. h>

\#define F_CPU 18432000UL // has a clock speed of 18.4MGH

\#include <util/delay.h> // uses F_CPU to provide delay routines

\#include <string.h>

\#include <stdlib.h>

\#include <stdio.h>

\#define ID 38

\#define SOLENOID_SLOWER 10 //for gettig 7200 per second

\#define EVENT_UNIT 512UL

\#define BUFFER_SIZE 32

\#define BAUD 38400

\#define MYUBRR F_CPU/16/BAUD-1

\#define SET_LED_PINS DDRD $\mid=(1<<$ DDD2 $) \mid(1<<$ DDD4 $) / /$ enable output pins

\#define LED_RED ( $1<<$ PD4)

\#define LED_RED_ON PORTD I $=$ LED_RED
\#define LED RED_OFF PORTD $\&=\sim$ LED RED

\#define LED_RED_TOGGLE PORTD $\stackrel{\wedge}{=}$ LED_RED

\#define LED_GREEN (1<<PD2)

\#define LED_GREEN_ON PORTD |= LED_GREEN

\#define LED_GREEN_OFF PORTD $\&=\sim$ LED_GREEN

\#define LED_GREEN_TOGGLE PORTD ^= LED_GREEN

\#define SET_OUTPUT_PINS_D DDRD $\mid=(1<<$ DDD3 $) \mid(1<<$ DDD5 $) \mid(1<<$ DDD6 $) \mid(1<<$ DDD7 $)$ //enable output pins

\#define SET_OUTPUT_PINS_B DDRB $\mid=(1<<$ DDBO $) \mid(1<<$ DDB1 $) \mid(1<<$ DDB2 $) / /$ enable output pins

\#define OUTPUTS 7

$\begin{array}{lll}\text { \#define OUTPUT_1 } & (1<<\text { PD3 }) \\ \text { \#define OUTPUT_2 } & (1<<\text { PD5 }) \\ \text { \#define OUTPUT_3 } & (1<<\text { PD6 }) \\ \text { \#define 0UTPUT_4 } & (1<<\text { PD7 }) \\ \text { \#define OUTPUT_5 } & (1<<\text { PB0 }) \\ \text { \#define 0UTPUT_6 } & (1<<\text { PB1) } \\ \text { \#define 0UTPUT_7 } & (1<<\text { PB2 })\end{array}$

\#define OUTPUT 7 (1<<PB2)

\#define OUTPUT 1 ON PORTD I= OUTPUT

\#define OUTPUT_1_OFF PORTD $\&=\sim$ OUTPUT

\#define OUTPUT_2_ON PORTD I= OUTPUT_2

\#define OUTPUT_2_OFF PORTD \&= OUTPUT_2

\#define OUTPUT_3_ON PORTD |= OUTPUT_3

\#define OUTPUT_3_OFF PORTD $\&=\sim$ OUTPUT 3

\#define OUTPUT 4 ON PORTD |= OUTPUT 4

\#define OUTPUT_4_OFF PORTD \&= OUTPUTT4

\#define OUTPUT 5 ON PORTB I= OUTPUT 5

\#define OUTPUT_5_OFF PORTB $\&=\sim$ OUTPUT_5

\#define OUTPUT_6_ON PORTB |= OUTPUT_6

\#define OUTPUT_6_OFF PORTB $\&=\sim$ OUTPUT' 6

\#define OUTPUT_7_ON PORTB |= OUTPUT_7

\#define 0UTPUT_7_OFF PORTB $\&=\sim 0 U T P U T+7$

typedef enum

NEW_MESSAGE_YES,

NEW MESSAGE NO

// There is a new message

NewMessagestate $t$

$/ /$ There is not a new message

typedef enum

SOLENOID_INTERRUPT_DIRTY_STATE,

SOLENOID_INTERRUPT_UPDATED_STATE

\} SolenoidInterruptState $t$;

// The values has to be updated

// The values hare updated

typedef enum

EVENT READY,

EVENT'DONE,

\} EventStäte_t 


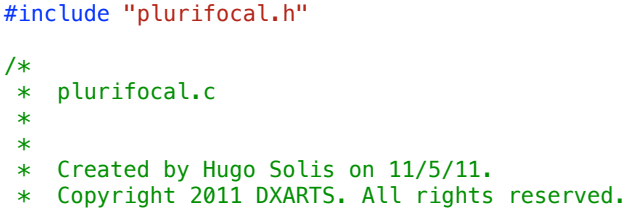




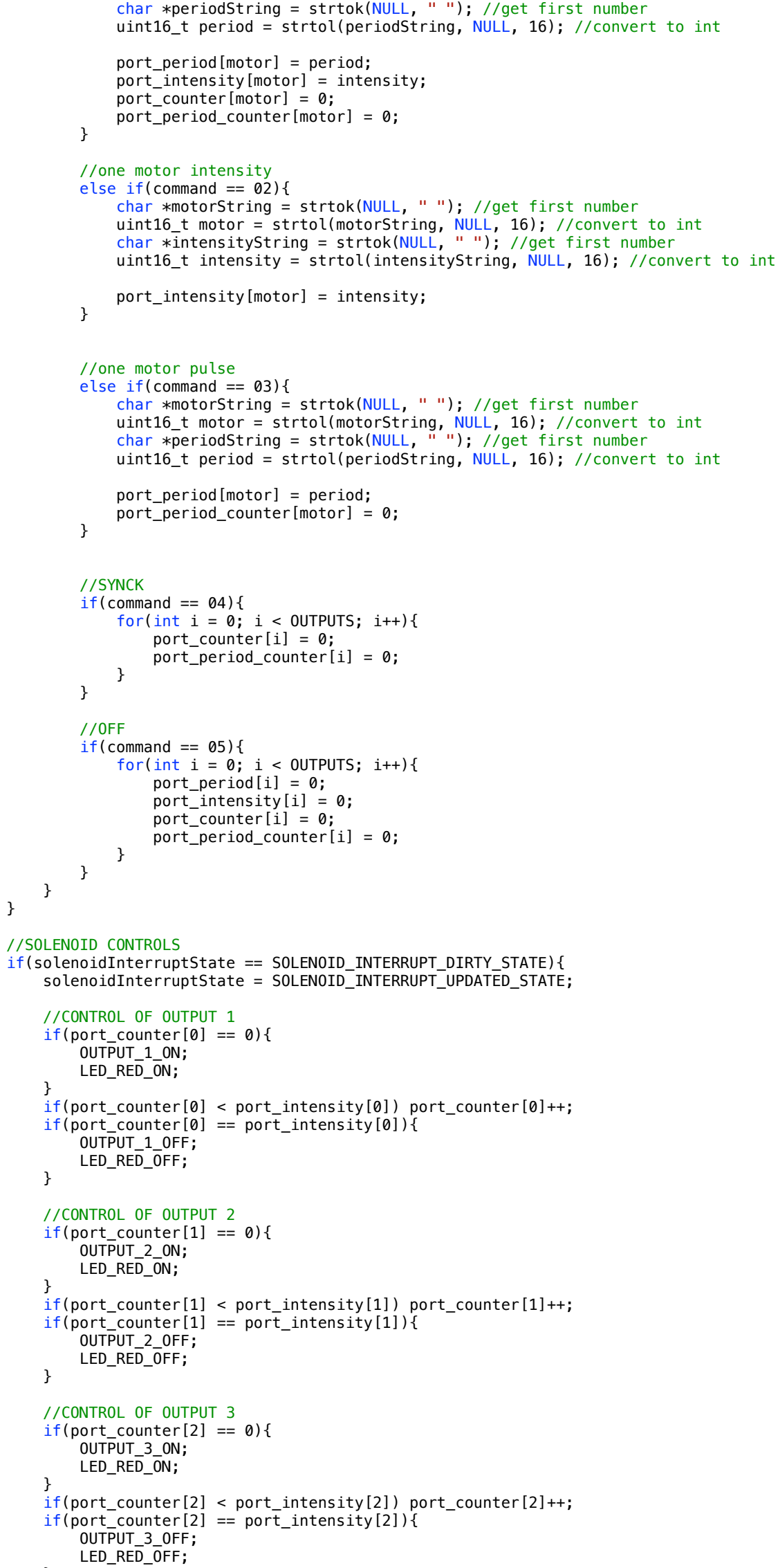




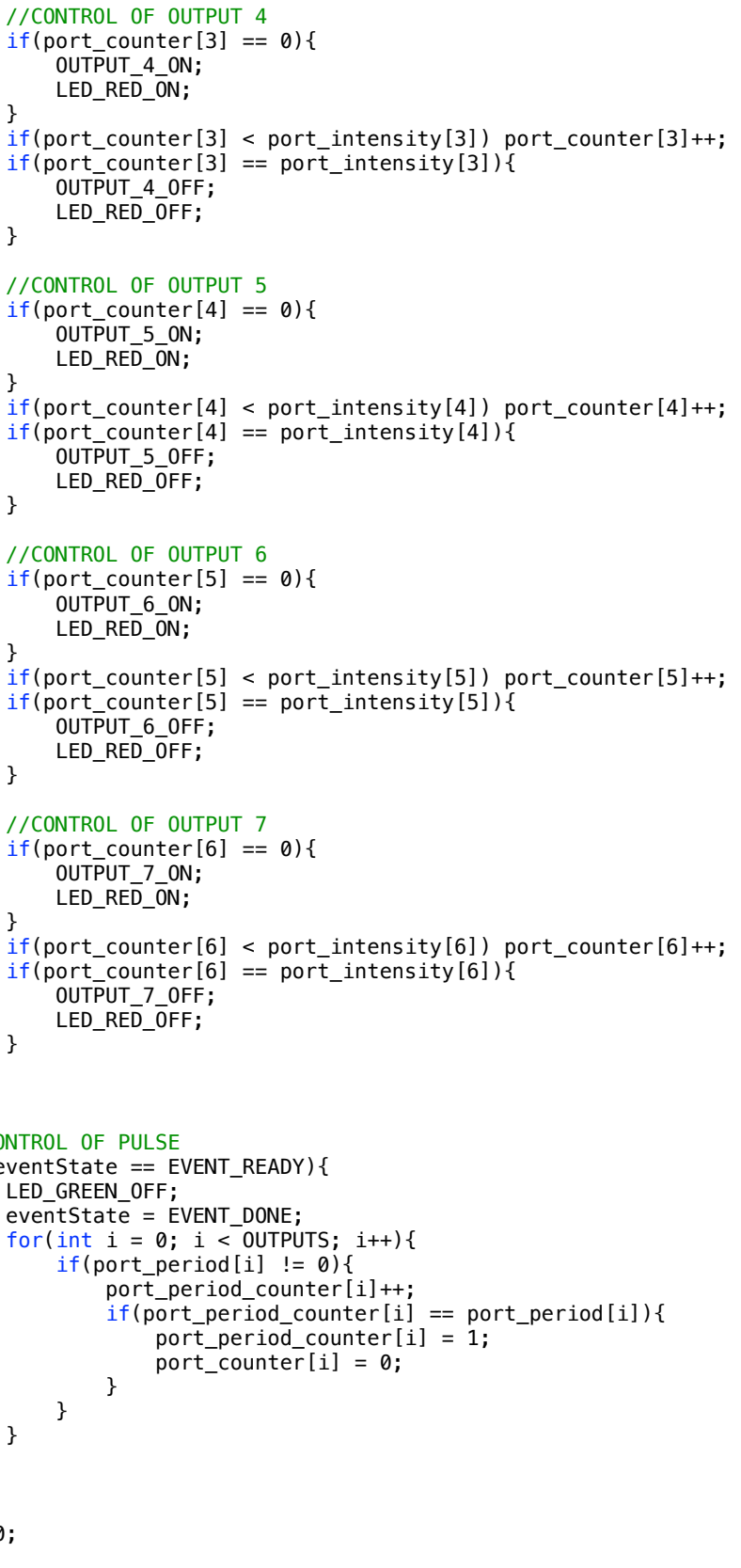

return 0

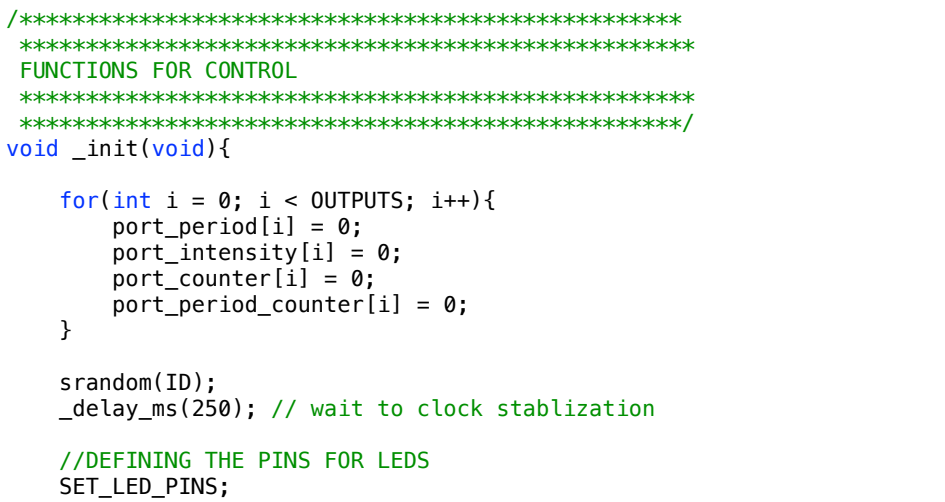


LED_RED_OFF;

LED_GREEN_OFF;

//DEFINIE THE PINS FOR DIGITAL OUTPUTS

SET_OUTPUT_PINS_B;

SET_OUTPUT_PINS_D;

//put outputs off

OUTPUT_1_OFF;

OUTPUT_2_OFF;

OUTPUT_3_OFF;

OUTPUT_4_OFF;

OUTPUT_5_OFF;

OUTPUT_6 OFF;

OUTPUT_7_OFF;

//TIMERO FOR SOLENOID RESOLUTION TIMES

TIMSKO $\mid=(1 \ll O C I E O A)$; //setting the compare match overflow $A$

TCCROB $\mid=(4 \ll$ CSOO); / / prescalar 256

OCROA = SOLENOID_SLOWER;

//TIMER1 FOR RYTHM

TIMSK1 $\mid=(1 \ll$ OCIE1A $) ; / /$ setting the compare match overflow A

TCCR1B $\mid=(5 \ll$ CS10); //prescalar 1024

\}

OCR1A = EVENT_UNIT;

ISR (TIMERO_COMPA_vect) \{

TCNTO = 0 ;

solenoidInterruptState $=$ SOLENOID_INTERRUPT_DIRTY_STATE;

\}

ISR (TIMER1_COMPA_vect) \{

TCNT1 = 0 ;

eventState = EVENT_READY;

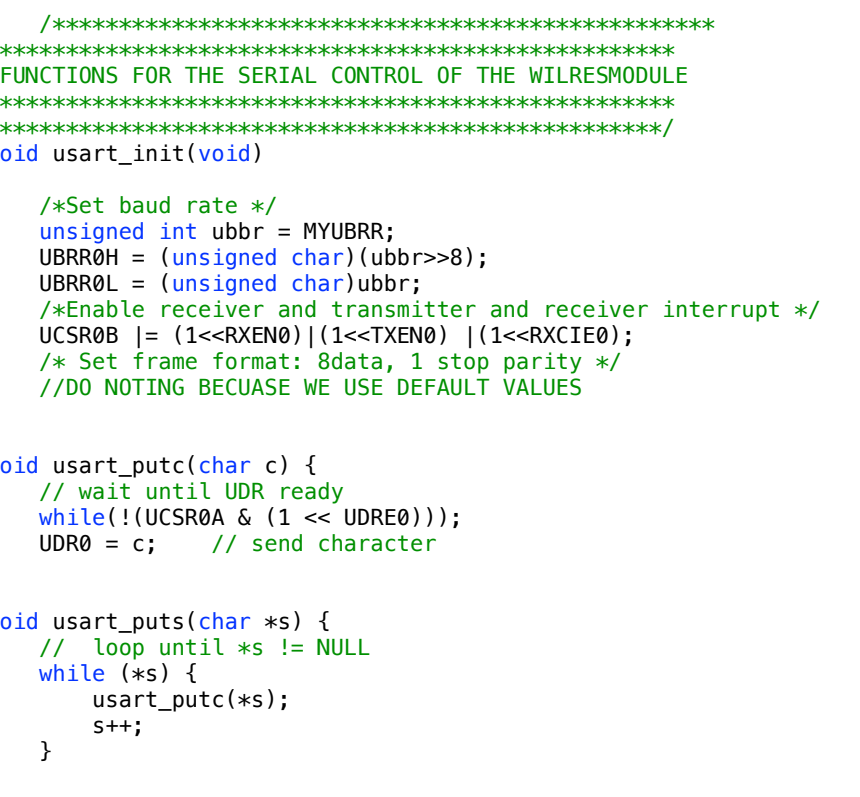

//USART receve

ISR(USART_RX_vect) \{

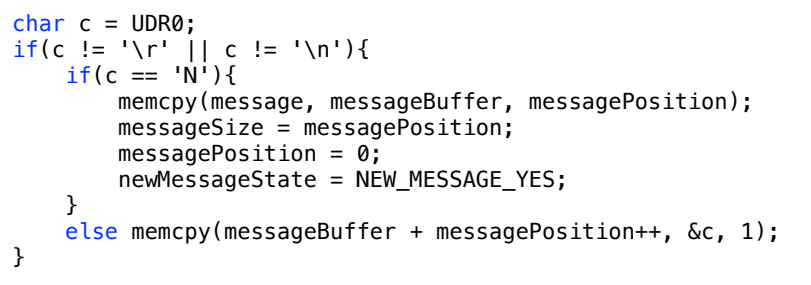




\begin{tabular}{l|l}
\hline word & description \\
\hline $\mathrm{XX}$ & $\begin{array}{l}\mathrm{XX} \text { is a number not used in the pec protocol but transmitted by the } \\
\text { Bitcloud protocol. } \\
\mathrm{YY}\end{array}$ \\
$\mathrm{YZ}$ is a hexadecimal number representing the module ID. If the message \\
$\begin{array}{l}\text { is OxFF the message is transmitted to all modules. } \\
\mathrm{ZZ} \text { is a hexadecimal number representing the command number. This } \\
\text { command is described in B.4. }\end{array}$ \\
\hline
\end{tabular}

Table B.3: Plurifocal Events Controller header protocol. 


\begin{tabular}{|c|c|}
\hline command & description \\
\hline $00 \mathrm{AA} B \mathrm{~B}$ & $\begin{array}{l}\text { ALL OUTPUTS SET INTENSITY AND PULSE. } 00 \text { command has two } \\
\text { parts. AA represents the intensity as a two-digit hexadecimal number. } \\
\text { BB sets the period between pulses. This message resets the starting } \\
\text { phase of the pulse. }\end{array}$ \\
\hline $01 \mathrm{AA} \mathrm{BB} \mathrm{CC}$ & $\begin{array}{l}\text { ONE MOTOR SET INTENSITY AND PULSE. } 01 \text { command has three } \\
\text { parts. AA represents the motor to be modified. BB sets the intensity } \\
\text { as a two-digit hexadecimal number. CC sets the period between pulses. } \\
\text { This message resets the starting phase of the pulse of the modified motor. }\end{array}$ \\
\hline $02 \mathrm{AA} B \mathrm{~B}$ & $\begin{array}{l}\text { ONE MOTOR SET INTENSITY. } 02 \text { command has two parts. AA } \\
\text { represents the motor to be modified. BB sets the intensity as a two- } \\
\text { digit hexadecimal number. This message does not reset the starting } \\
\text { phase of the pulse of the modified motor. }\end{array}$ \\
\hline $03 \mathrm{AA} B \mathrm{~B}$ & $\begin{array}{l}\text { ONE MOTOR SET PULSE. } 03 \text { command has two parts. AA represents } \\
\text { the motor to be modified. BB sets the pulse as a two-digit hexadecimal } \\
\text { number. This message resets the starting phase of the pulse of the } \\
\text { modified motor but not the intensity. }\end{array}$ \\
\hline 8704 & $\begin{array}{l}\text { RESETS PULSE PHASE. } 04 \text { command does not have more words. This } \\
\text { message resets the pulse phase of all motors allowing to resync rhythmic } \\
\text { patterns. }\end{array}$ \\
\hline 2205 & $\begin{array}{l}\text { OFF PARAMETERS. } 05 \text { command does not have more words. This } \\
\text { message sets the intensity to zero and stops the pulse patterns setting } \\
\text { all motors to off. }\end{array}$ \\
\hline
\end{tabular}

Table B.4: Plurifocal Events Controller body protocol. 


\begin{tabular}{|c|c|}
\hline command & description \\
\hline $67010030 \mathrm{~F} 0$ & $\begin{array}{l}\text { This message would set all motors of module } 01 \text { to intensity } \\
\text { of } 48(0 \times 30) \text { and pulse of } 240(0 \times \mathrm{F} 0) \text {. }\end{array}$ \\
\hline $87 \mathrm{FF} 04$ & This message would reset phase of all motors of all modules. \\
\hline $45090104 \mathrm{FF} 90$ & $\begin{array}{l}\text { This message would set motor id } 4 \text { of module id } 09 \text { to inten- } \\
\text { sity of value } 255(0 \mathrm{xFF}) \text { and pulse of } 144(0 \times 90) \text {. }\end{array}$ \\
\hline 2310020309 & $\begin{array}{l}\text { This message would set motor id } 3 \text { of module id } 10 \text { to inten- } \\
\text { sity of value } 09 \text {. }\end{array}$ \\
\hline 780005 & This message would turn off module 05 . \\
\hline 99 FF 030210 & $\begin{array}{l}\text { This message would set the pulse of motor id } 02 \text { of all mod- } \\
\text { ules to value } 10 \text {. }\end{array}$ \\
\hline
\end{tabular}

Table B.5: Plurifocal Events Controller body protocol examples. 


\section{Appendix C \\ JUUM SCORES}

The following images show screenshots of different digital scores created with the Juum software. These images give and idea of the structure, shape, and organization of each artwork. The pieces presented are:

Axial The first screenshot shows the score of the final version of the Axial work. The upper section shows the control of solenoids; the lower region shows the starting point for audio files. The central line represents the audio mixing control.

Tokyo's concerts The second screenshot presents a testing window used for control of DC motors during the concerts in Tokyo. This version demonstrates graphically the distribution of the different motors along the space. This particular screenshot shows twelve motors spread over two floors.

Conjuro de la Memoria The third screenshot shows the structure of the Conjuro de la Memoria concert. This piece, for prerecorded voices, piano sounds, viola sounds, and live piano and viola, has a duration of 45 minutes. The score coordinates the different layers of sound at the same time, which allows for the sound spatialization of the viola player. The temporal element (circular object) is used to represent the physical location of the viola player in the hall.

Piano solo The fourth screenshot shows the material used during the piano improvisation at the Chapel Performance Space. The material presents a long audio control object and several short elements where different kind of audio processes and variations are generated using the MIDI information registered with a PianoBar ${ }^{1}$.

\footnotetext{
${ }^{1}$ The PianoBar is a device, invented by D. Buchla in 2001 and commercialized by Moog, which reads movement from the keys of a regular acoustic piano and translates the movement into MIDI format. The device has since been discontinued.
} 
Just a Minute The fifth screenshot presents the score used during the Just a Minute a collaboration with Maria Cynthia Anderson and Devin McDermott, as part of the $3 \mathrm{D}$ concert. Seven sections of less than a minute comprise the piece. Since the specific duration of each section could vary on each execution, the point in time can be manually updated using numbers on the computer keyboard. For this performance Juum controlled the video transformations of a real-time video feed pointed to the dancer at the same time that generated electronic ambisonics sounds based on the MIDI input.

Piece for Computer and Viola The final screenshot presents the first use of Juum in a concert setting. Juum was used to generate a basic visual background at the same time that it generated sound synthesis based on the 2D plane-projection of a 3D trajectory. This version did not yet have the final graphical template and had a very limited control pallet. 


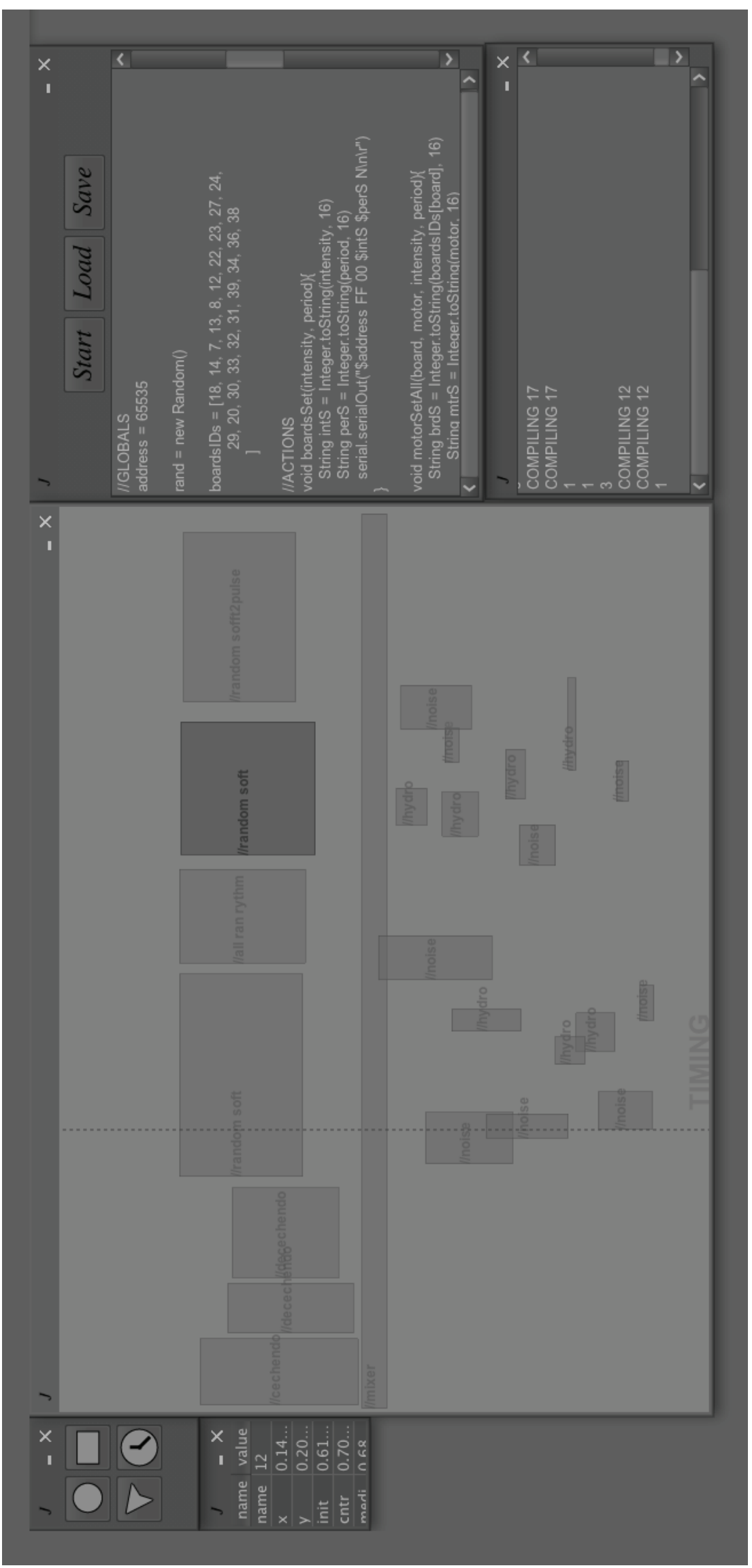




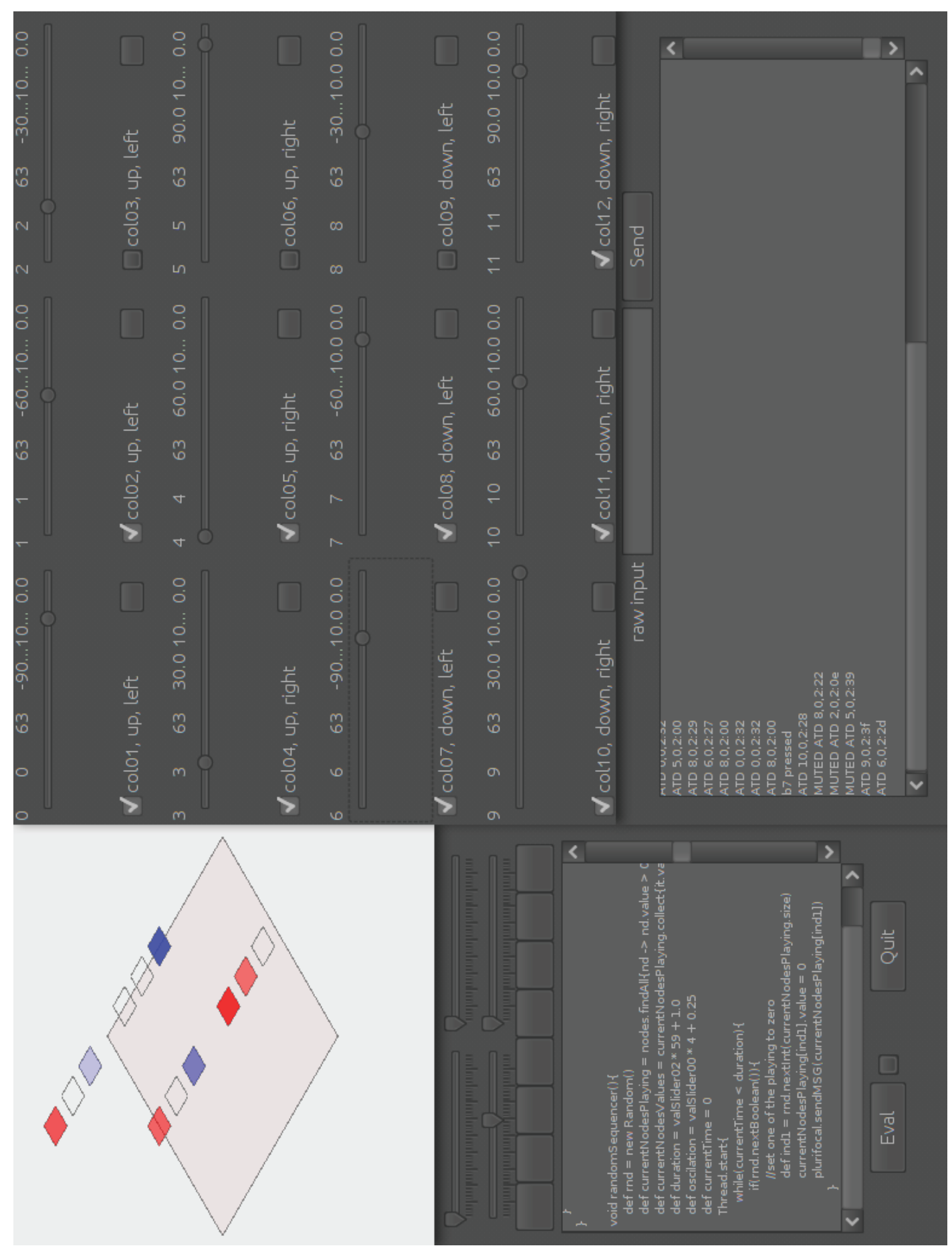




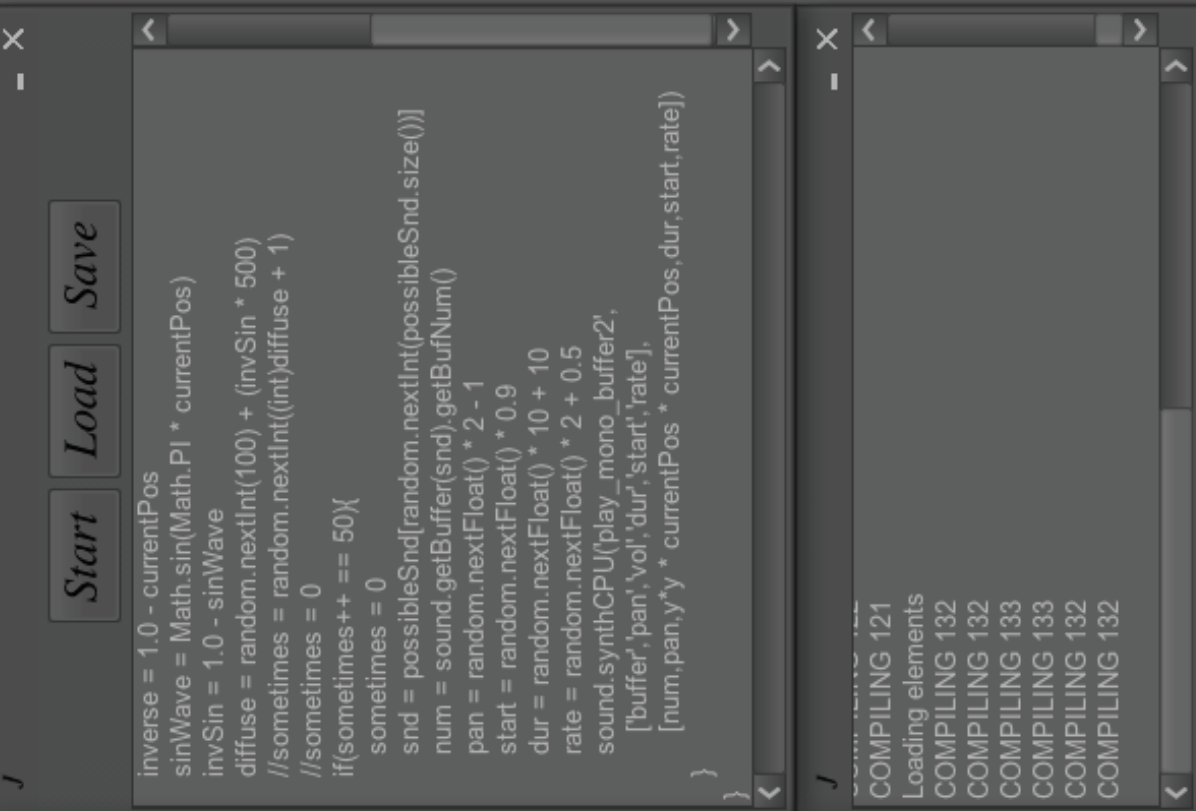

I 


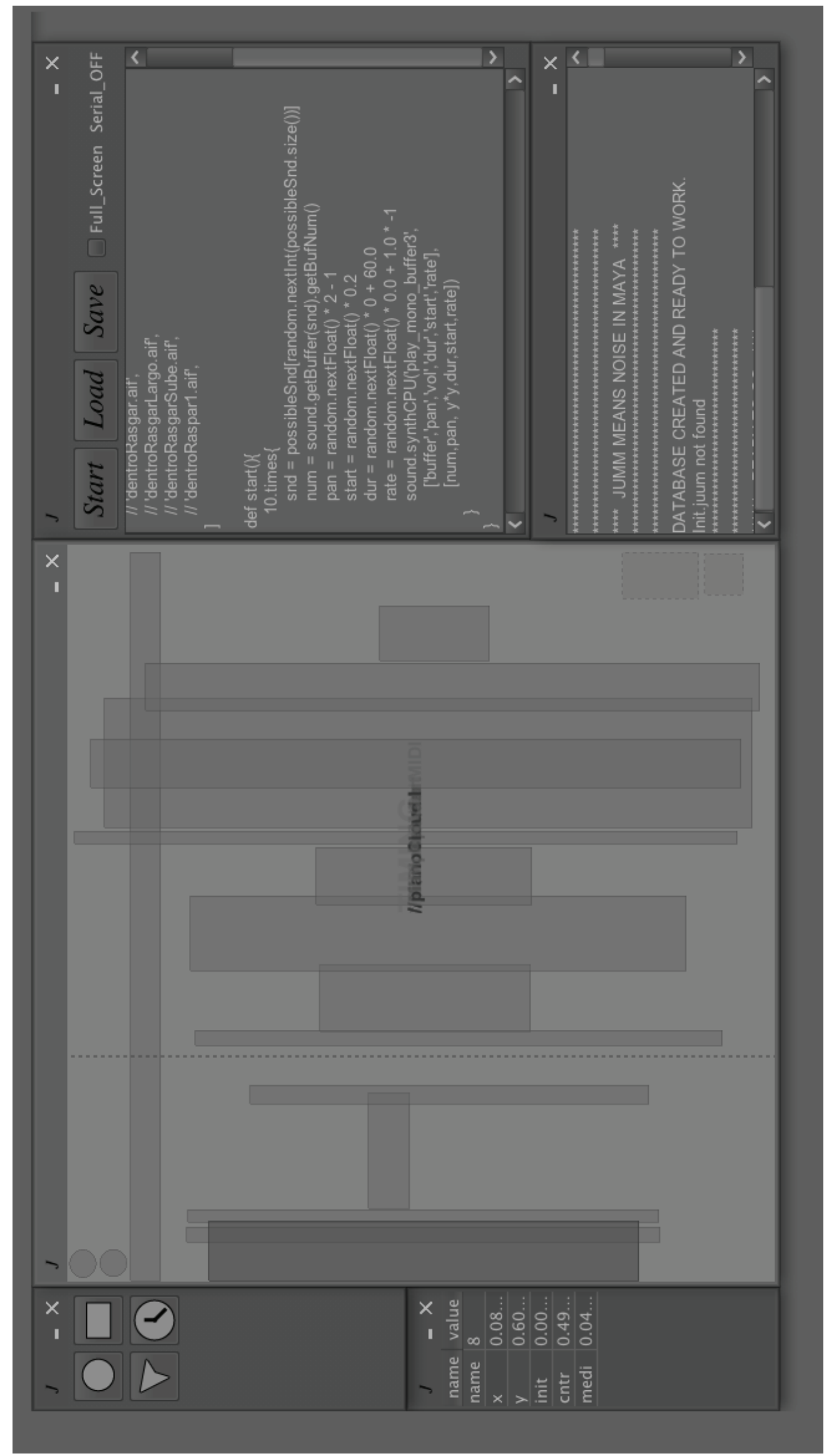




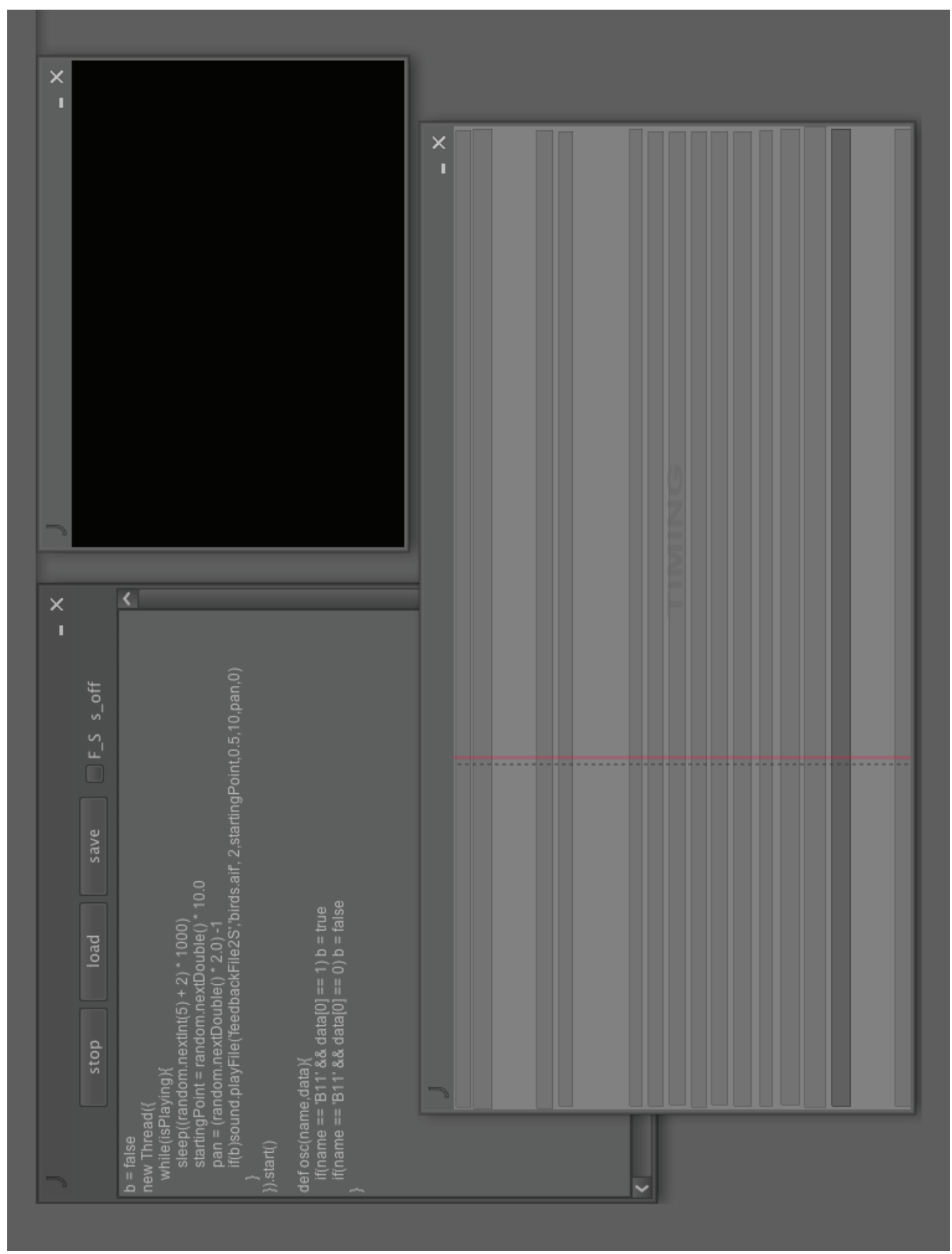




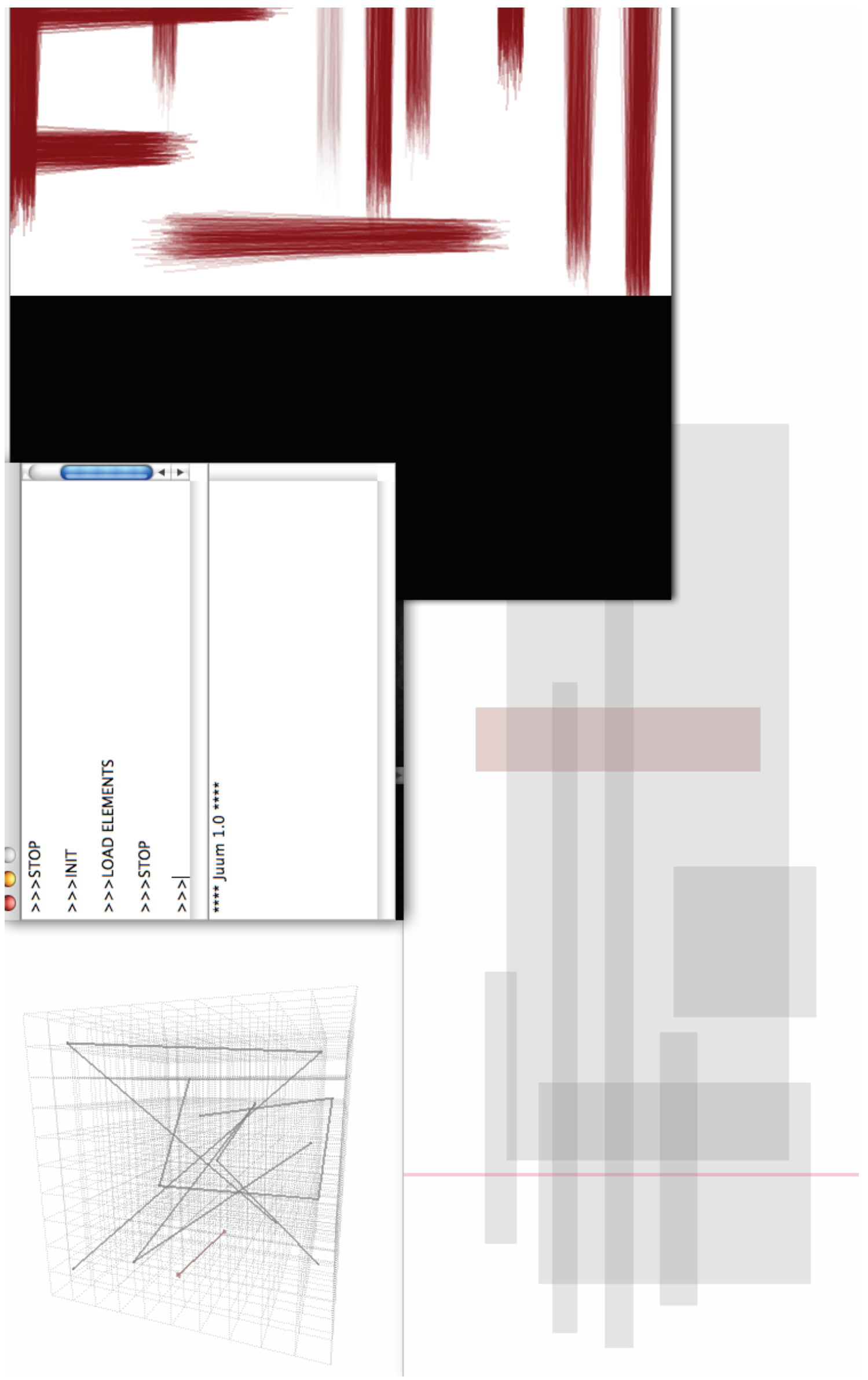




\section{Appendix D}

\section{CLOUD SYNTHESIS}

\section{D.1 Amazon Web Services}

Amazon offers a set of computation tools under the name of Amazon Web Services (http://aws.amazon.com/). Among these services, the Elastic Computer Cloud (E2) provides virtual servers of different sizes and CPUs. The servers can be customized and later saved as images from which other instances can be created. The charge for these servers is by the hour but the cost is not high. The Simple Storage Service (S3) provides a data storage service through a web services interface. Amazon charges users monthly on a gigabyte and bandwidth basis for sending or receiving data. The Amazon SimpleDB allows customers to run queries on structured data. It provides the database functionality to control and access the E2 and S3 infrastructure. The Amazon Simple Queue Service (SQS) is a hosted message queue for web applications that allows users to communicate and send data between different elements of a cloud system, with delivery guaranteed between components of the infrastructure.

\section{D.2 Grails}

Grails (http://grails.org/) is a web application framework that follows the coding by convention paradigm. It works to hide the configuration details from the developer and to quickly create full web applications. Grails is similar to Ruby on Rails but uses the Groovy (http://groovy. codehaus.org/) programming language instead of Ruby ${ }^{1}$.

\section{D.3 The Cloud Synthesis Infrastructure}

The first step in creating the operational system was to generate a server instance that could render sound. The SuperCollider source code was downloaded to one running instance using the preinstalled curl command. SuperCollider was then complied and

\footnotetext{
${ }^{1}$ The author started to study Grails during the mechatronic course as a means to coordinate distributed actions.
} 
the appropriated folders created for placing the classes and synth definitions. Java and Groovy were also installed on this server, as well as a set of Groovy scripts that allows to run the server of SuperCollider. These scripts handle the connection with the S3 data storage, allowing for retrieval of oceanographic data, audio data and, once the render was finished, putting the audio result into the S3 storage location. The instance was then saved as a EBS image for future duplications.

Another image was created that also has Java and Groovy. This image does not render audio but works as the coordinator of the audio images and also works as a web server for user interaction. Grails was installed on this image and a basic Grails application was created that allows users to select types of processes and source materials. Using SQS messaging, this server knew how many renders were in the queue, turning off all sound-render servers if the list of render sounds was empty and turning on soundrender servers if the list grew. The balance between speed of rendering and number of sound servers (cost) was selectable by setting the maximum amount of elements in the queue.

With this structure, just one small server was constantly running and the CPUintensive audio servers turn on only when needed. While never required, this technique would allow for the activation of as many servers as needed in order to maximize sound processing speed. The data sources for sonification were centralized into a single location, keeping the required space as small as possible. After being rendered, sounds were immediately accessible with a web link. The entire logic of the system is presented in a diagram at the end of this appendix.

The following code part of the Grails application counts the amount of the sound instances running in the system. The entire source code is contained in the cloudSynthesis.zip file in the Digital Materials.

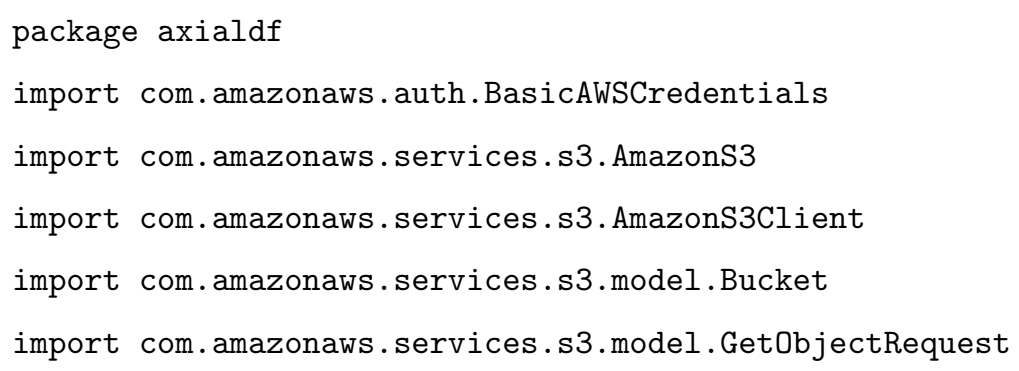




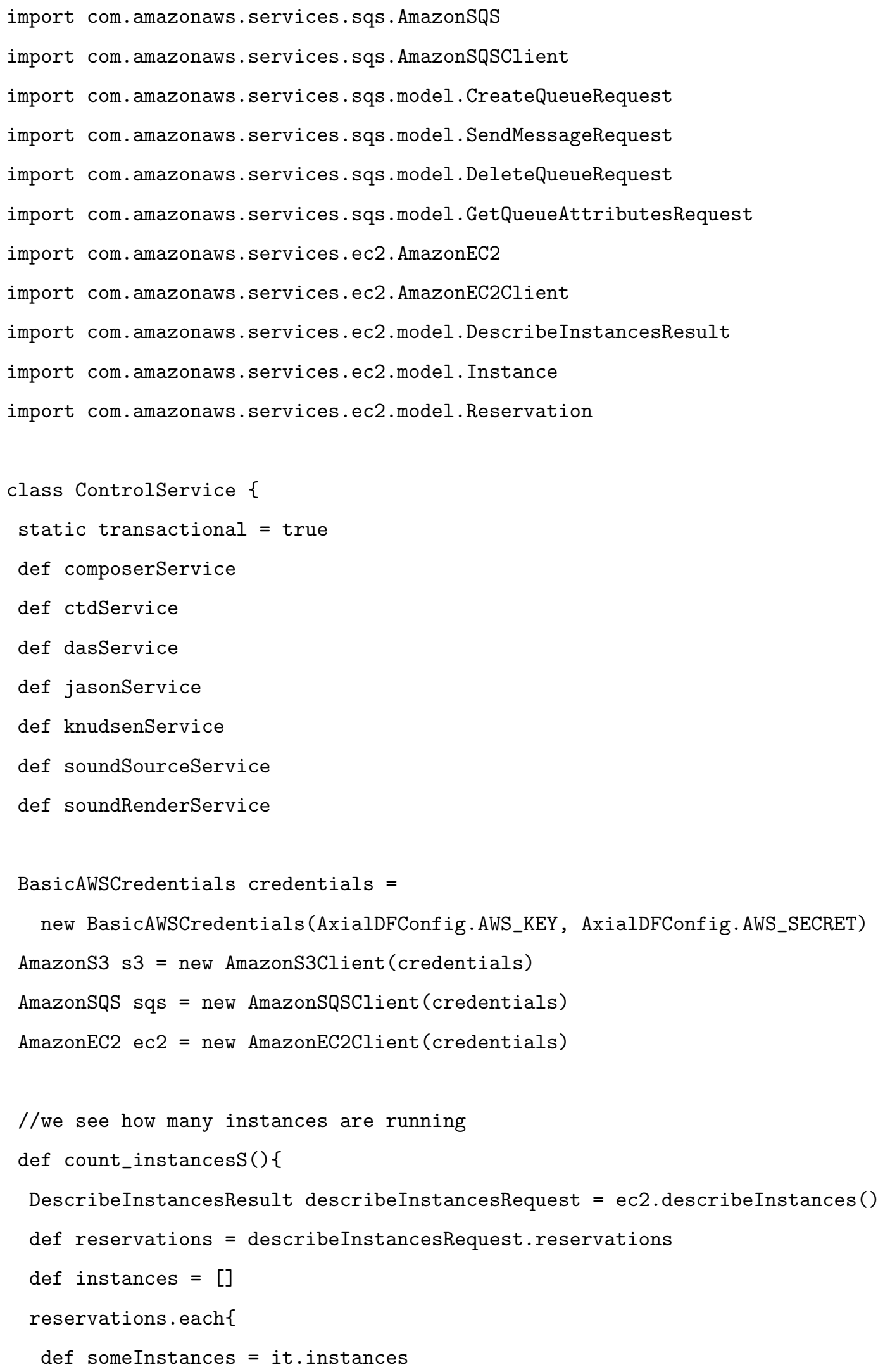




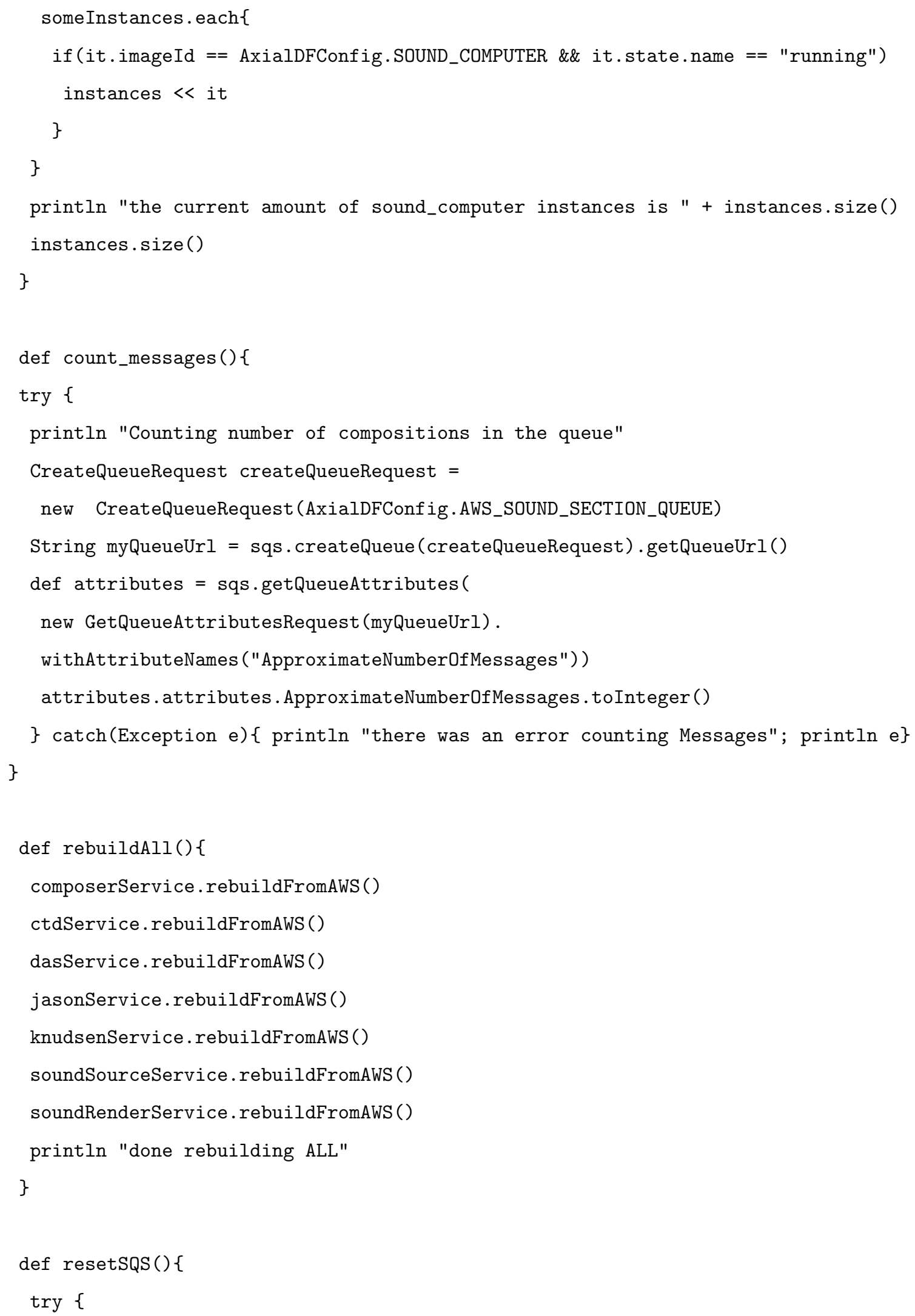




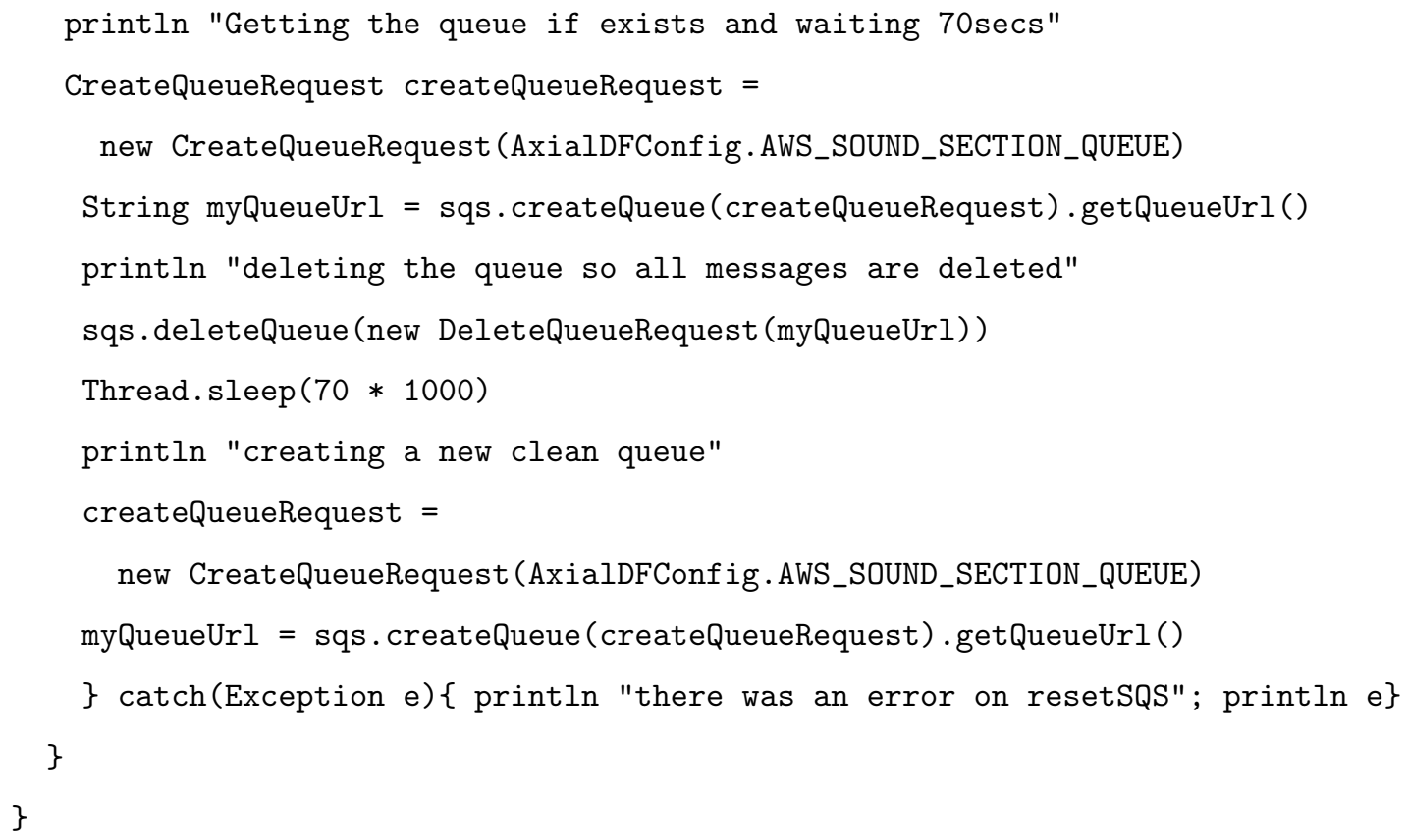

\section{D.4 Audio processes}

The architecture of the system allows for the generation of small processing scripts that work as Plugins. These small pieces of code contains the processing logic and can be copied into a particular directory. Once loaded, the system has them as a processing option in the list of audio rendering processes. This mechanism helped to keep the architecture in one part and sound logic in another part.

The following code corresponds to the main script that each audio instance run when the computer initiated. The script searches for materials that have not been rendered and if they exist, the script downloads all the required materials, including the composer script, and runs the entire process.

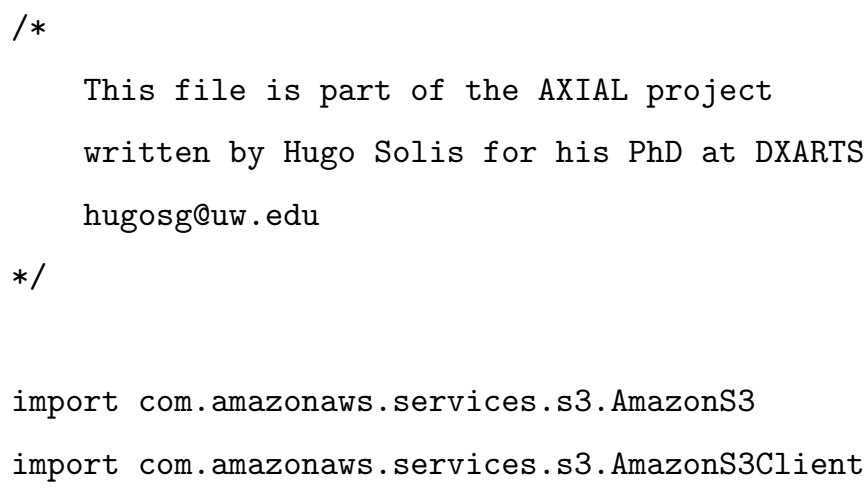




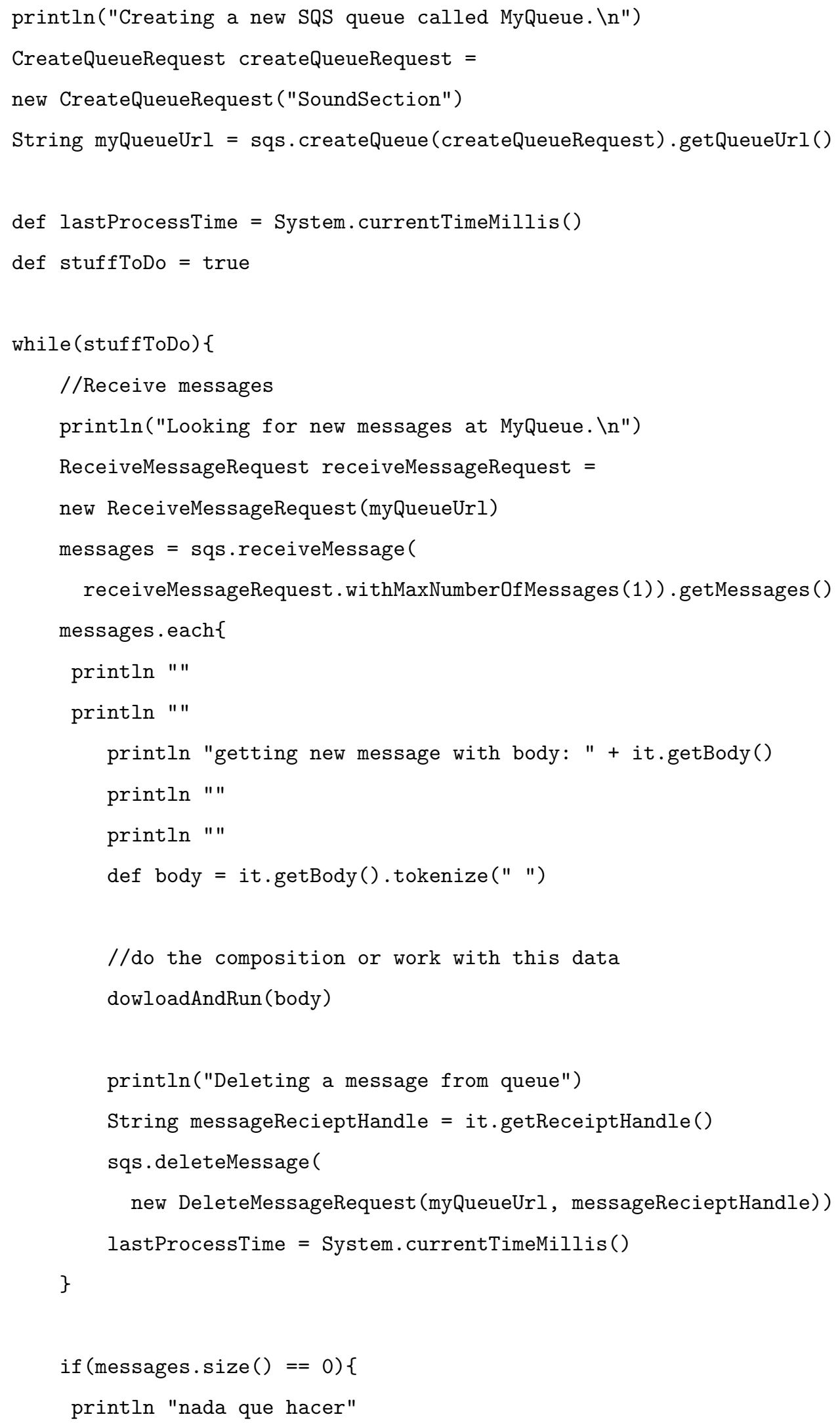




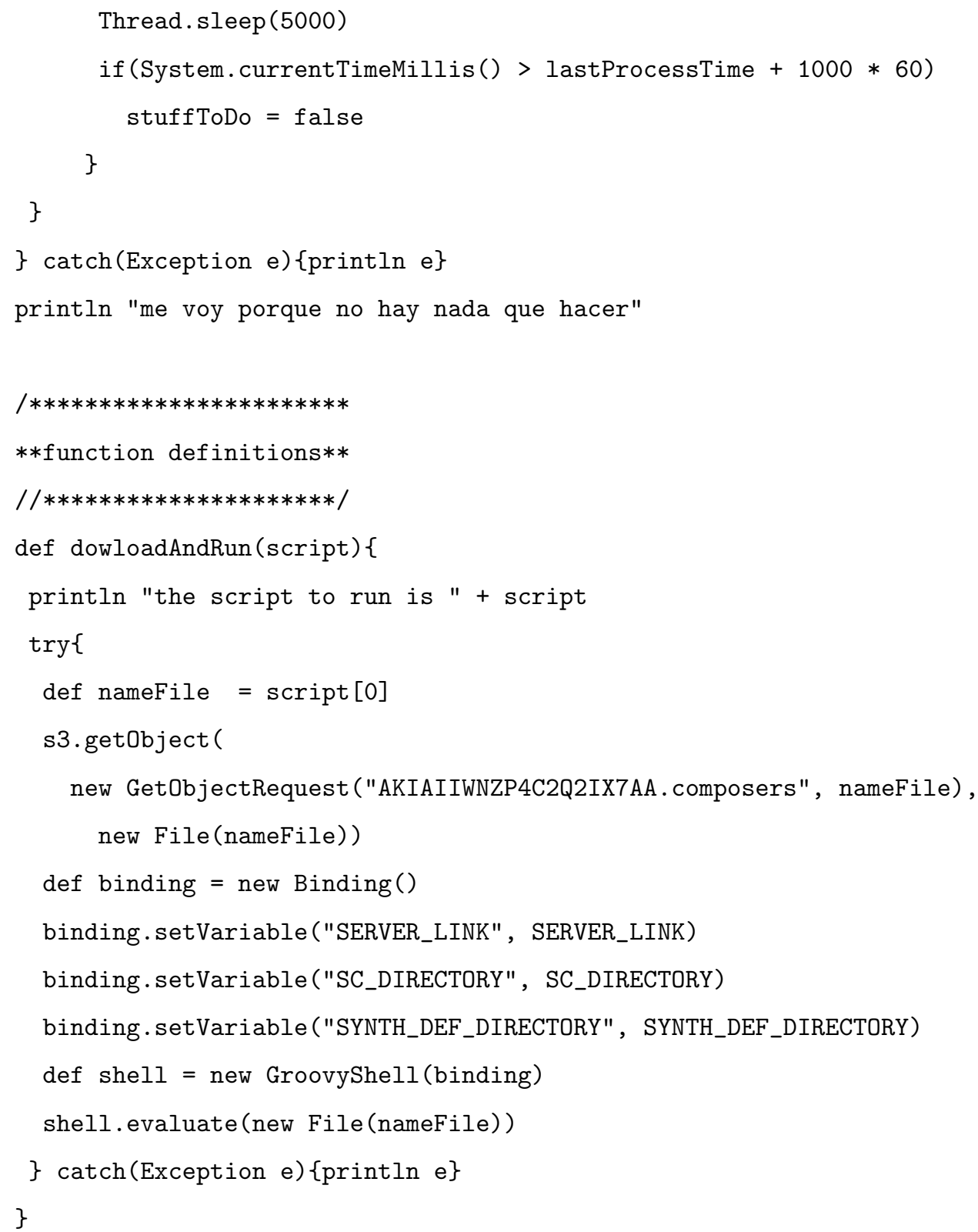

The following code shows a Groovy script that works as a plugin for the system. It loads a set of data, generates a score, renders the score running the SuperCollider server, uploads the sound and notifies the databases. This particular version uses the EchoNest API to generate sound segmentation-based perceptual changes of acoustic regions ${ }^{2}$.

${ }^{2}$ The EchoNest API (http://echonest.com/) offers a mechanism to obtain audio descriptions of musical pieces. It was used as an off-the-shelf mechanism to get information about audio recordings. For a more robust and self-contained system it would be much better to write the code in order to locally generate the analysis. 
import com.amazonaws.auth.BasicAWSCredentials

import com.amazonaws.services.s3.AmazonS3

import com.amazonaws.services.s3.AmazonS3Client

import com.amazonaws.services.s3.model.Bucket

import com.amazonaws.services.s3.model. CannedAccessControlList

import com.amazonaws.services.s3.model.GetObjectRequest

import com.amazonaws.services.s3.model.PutObjectRequest

import com.amazonaws.services.simpledb.AmazonSimpleDB

import com.amazonaws.services.simpledb.AmazonSimpleDBClient

import com.amazonaws.services.simpledb.model.Attribute

import com.amazonaws.services.simpledb.model. PutAttributesRequest

import com.amazonaws.services.simpledb.model.CreateDomainRequest

import com.amazonaws.services.simpledb.model.DeleteAttributesRequest

import com.amazonaws.services.simpledb.model.DeleteDomainRequest

import com.amazonaws.services.simpledb.model.Item

import com.amazonaws.services.simpledb.model. PutAttributesRequest

import com.amazonaws.services.simpledb.model.ReplaceableAttribute as RA

import com.amazonaws.services.simpledb.model.ReplaceableItem

import com.amazonaws.services.simpledb.model.SelectRequest

import groovyx.net.http.RESTClient

import groovy.util.slurpersupport.GPathResult

import static groovyx.net.http. ContentType.URLENC

import com.echonest.api.v4.EchoNestAPI

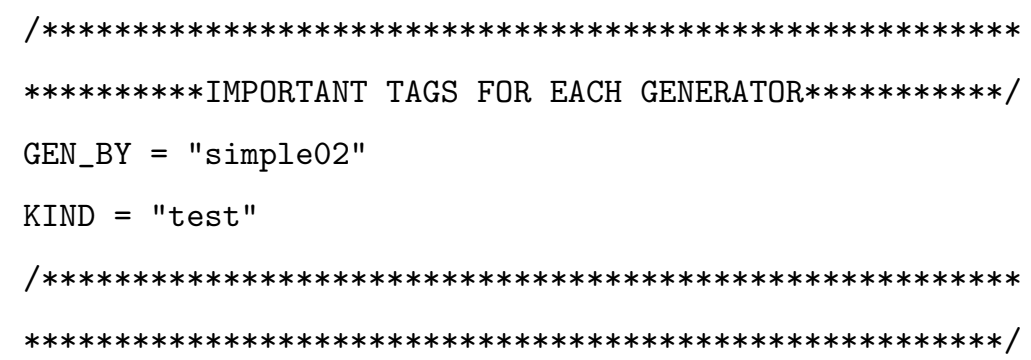

//THESE 3 GLOBALS COMES FROM THE BINDING THAT RUNS THE SCRIPT//

//SERVER_LINK = args[0] //here in the web is our mainwebApp 


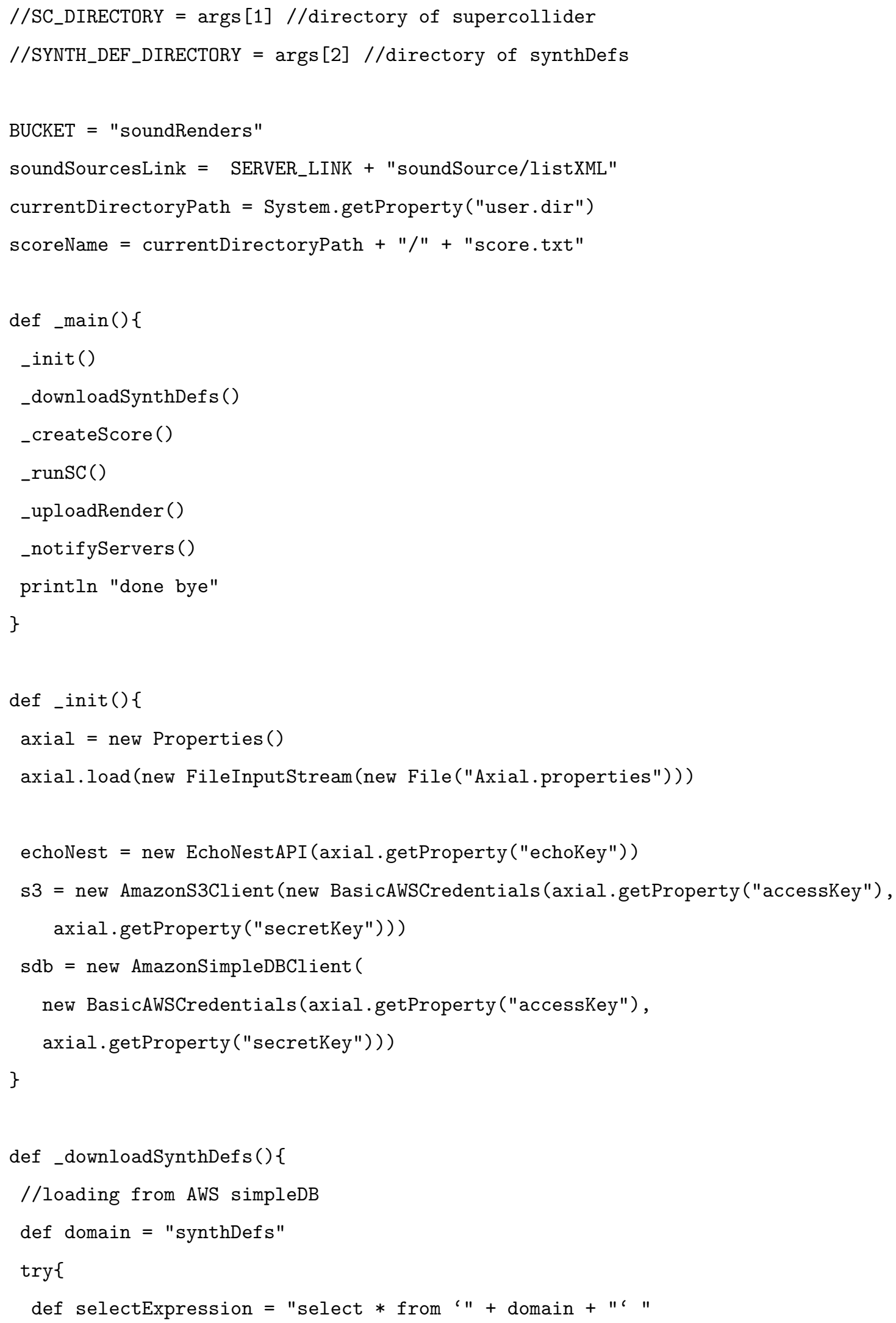




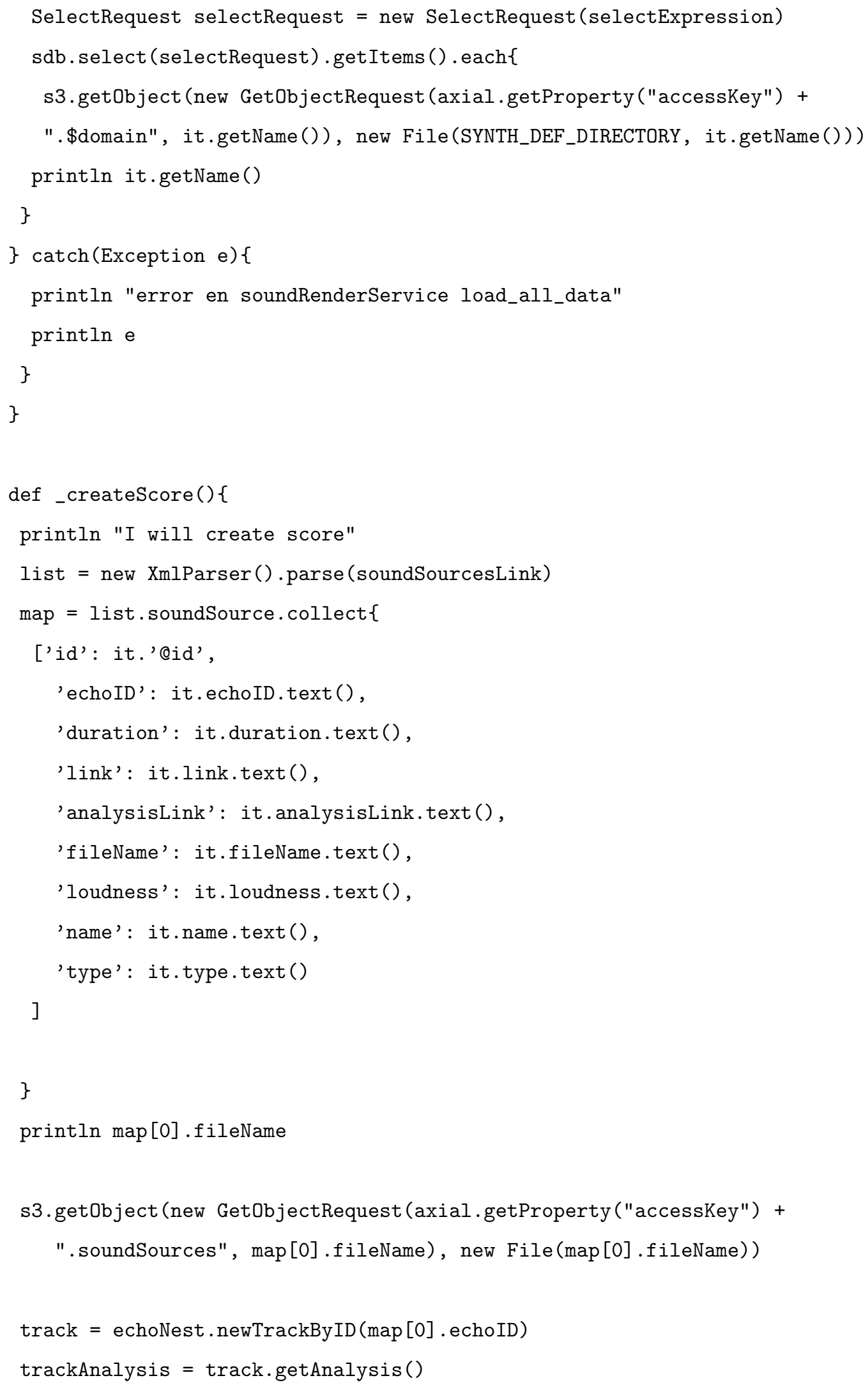




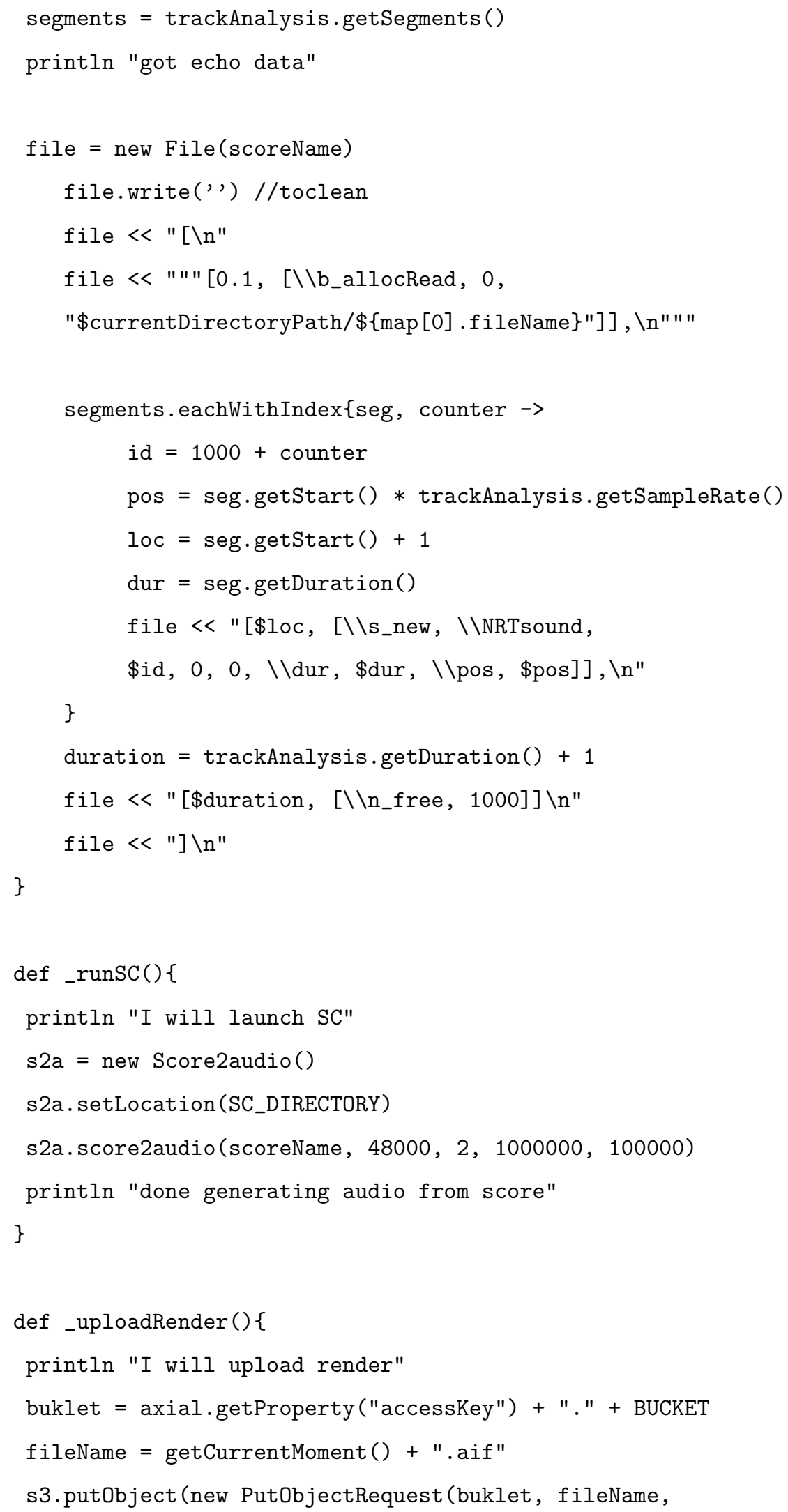




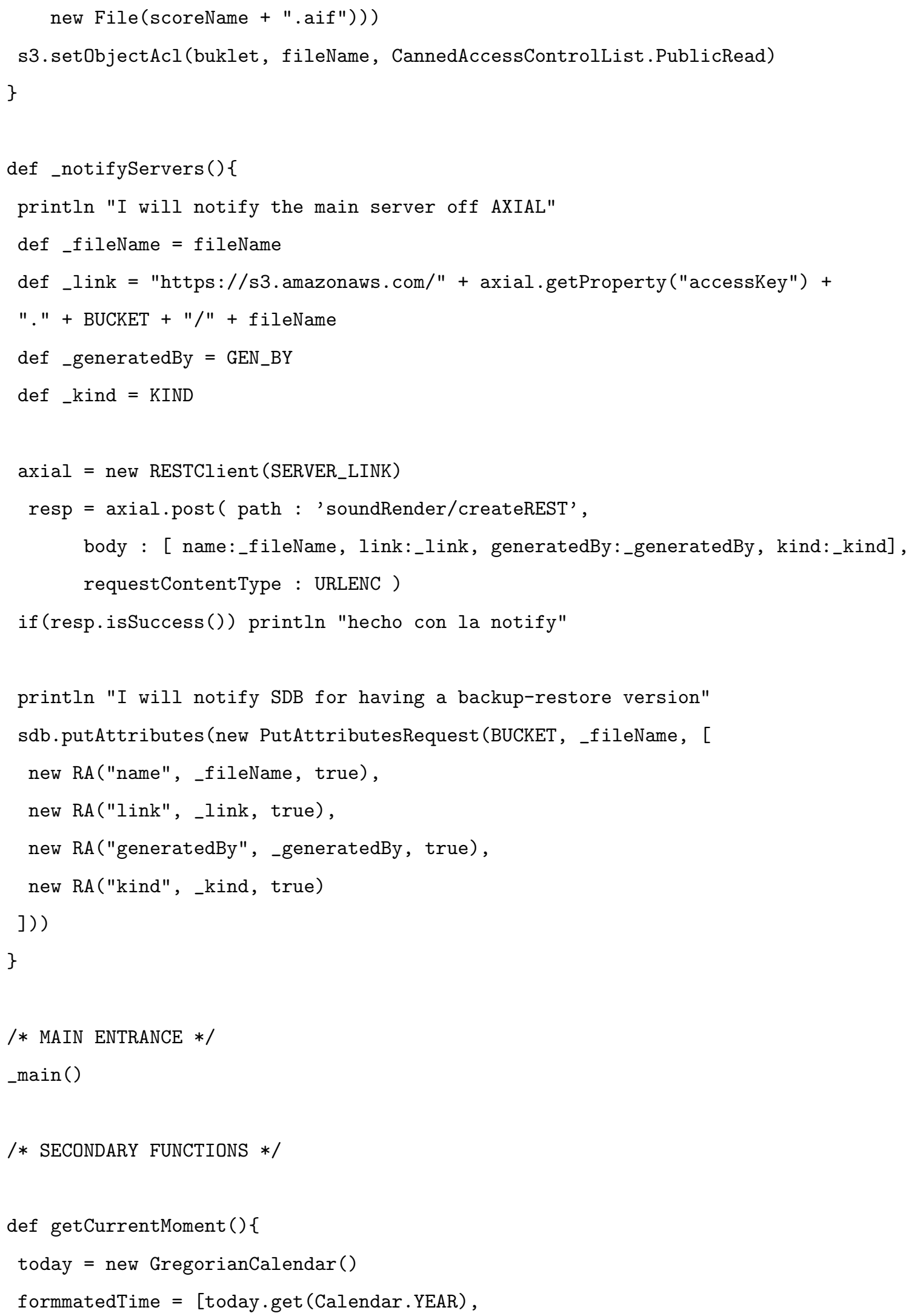


today .get (Calendar.DAY_OF_YEAR),

today.get (Calendar.HOUR_OF_DAY),

today .get (Calendar.MINUTE),

today.get (Calendar.SECOND)] . join("_")

\} 


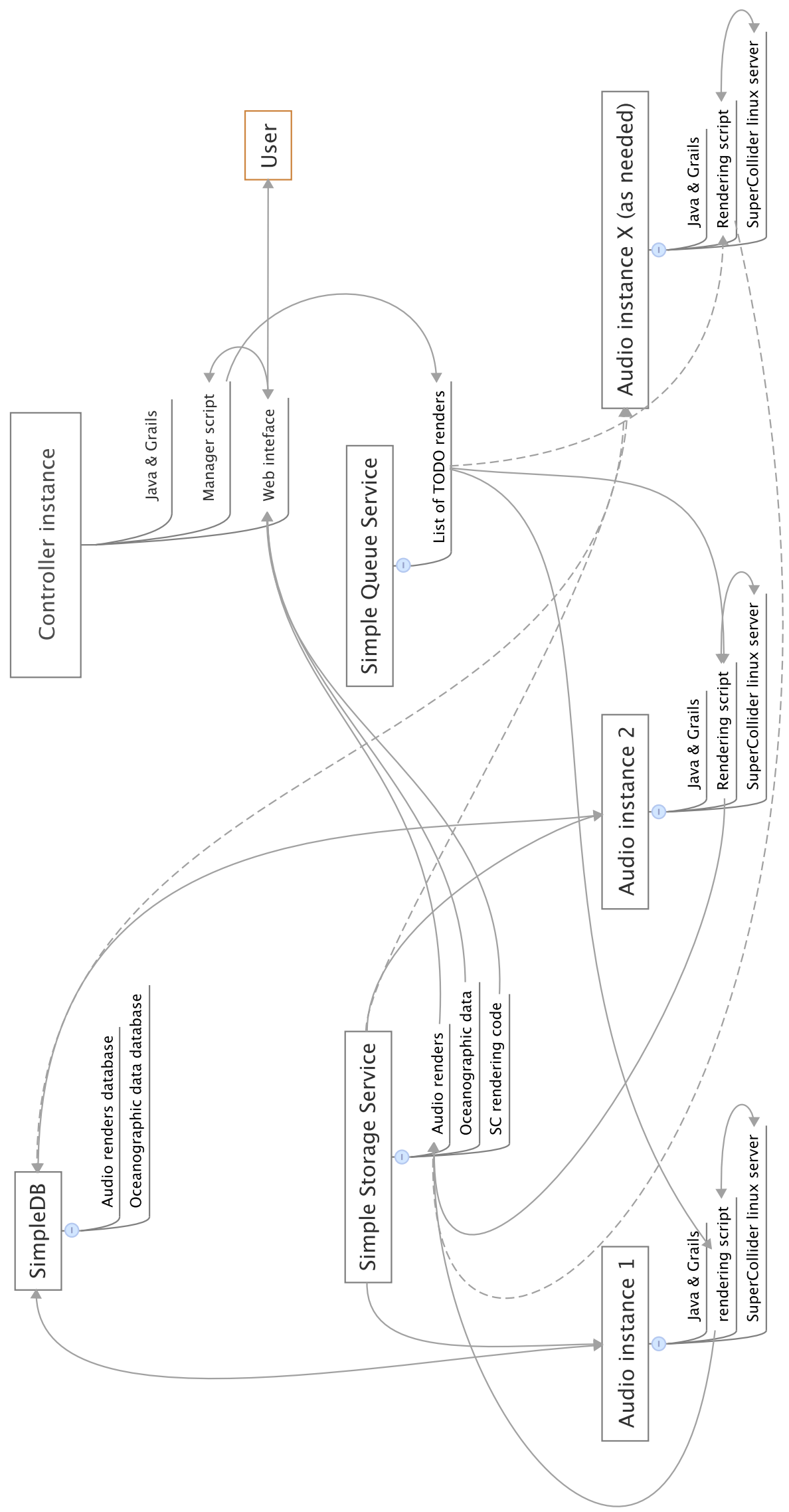




\section{Appendix E \\ MARINE GEOLOGY CONTEXT AND THE OCEAN OBSERVATORY INITIATIVE}

This chapter offers an introduction to elements and knowledge about the deep sea that were used to generate the sound installation's logic. The idea is to give a basic vocabulary that can help to frame the inner logic and artistic decisions of the sound installation $^{1}$. Also, this chapter presents a detailed description of the Oceans Observatory Initiative, an ongoing and ambitious research project that, using the most advanced technology available, actualizes an entire new way of studying the ocean by deploying a network of fully interconnected devices capable of sending constant data to land. The information will be available for researchers and general audiences for studying and exploring ocean behavior with a time/space resolution never before available. ${ }^{2}$.

\section{E.1 Art, Ocean, and Myths}

One of the most poetical images in all of human history is the sea, and innumerable artistic works employ the sea as their primary subject. The reason is assuredly simple: the sea has been there since we emerged as humans, and for millenia it was the limit of our space, the clear boundary of our surroundings, and has always possessed an overwhelming power that no human effort can match. There is an ontological relationship between the sea and the human, a dichotomy of sensations based on fear and thankfulness. With its force, dimension, and inhability by mankind, the sea establishes our human proportions and limits. On the other hand, the power and mystic nature of the sea has fed cultures for centuries; humanity therefore is thankful to this precious and exuberant liquid. For thousands years, in the human imagination the sea was an

\footnotetext{
${ }^{1}$ This chapter summarizes the first six chapters of the book An introduction to the worlds oceans[38]. Several sentences are literal quotations or modification of the original.

${ }^{2}$ This chapter summarizes the content of the web pages http://www.interactiveoceans. washington.edu and http://www.oceanobservatories.org. Several sentences are literal quotations or modifications of the original sources.
} 


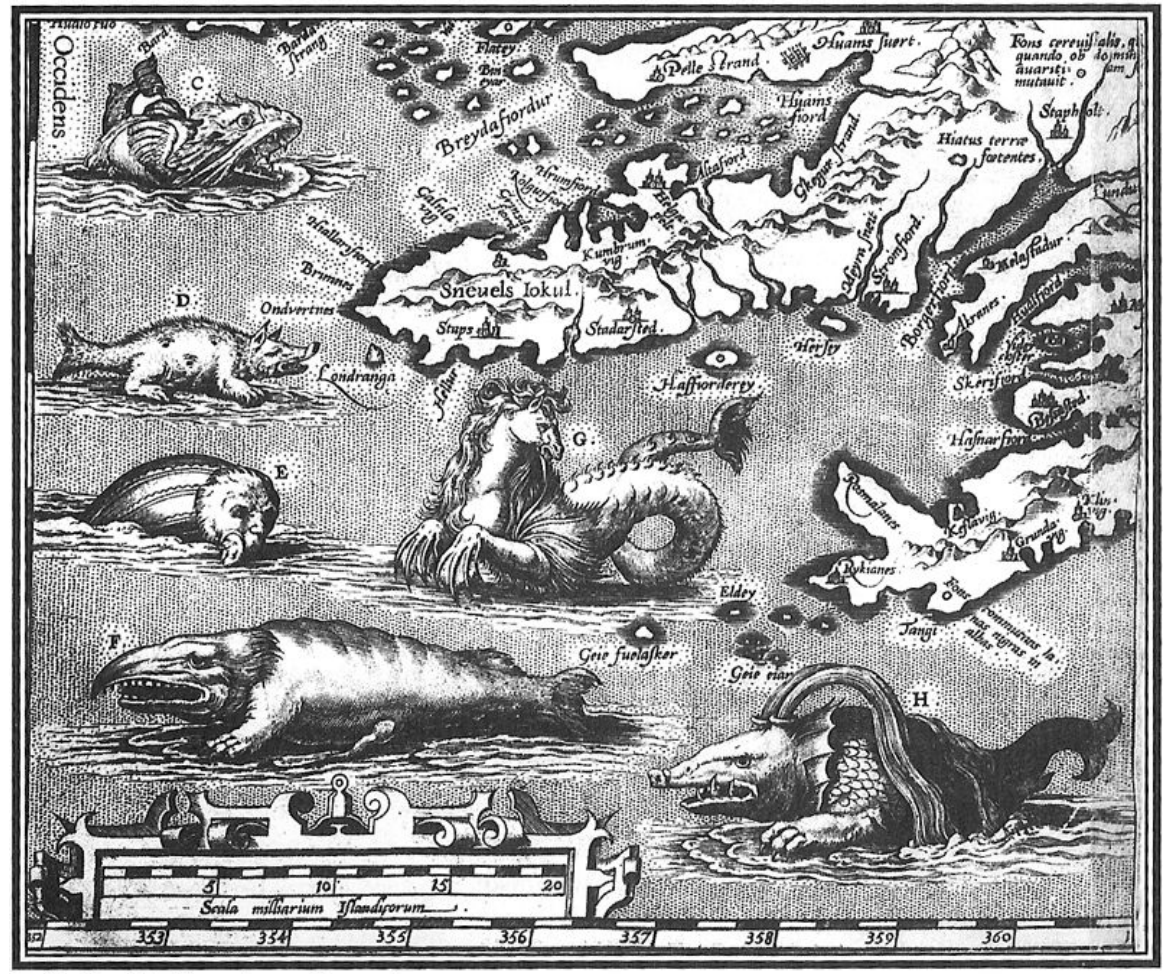

Provided by: http://www.strangescience.net

Originally published in: Theatrum Orbis Terrarum
Now appears in: Monsters of the Sea by Richard Ellis

Figure E.1: Theatrum Orbis Terrarum.

impenetrable space inhabited by gods, monsters, and beasts. According to the Bible "the waters" was one of the first concepts created by God. Greek mythology has Poseidon and Roman mythology has Neptune as their gods of the sea. In Aztec mythology, Acuecucyoticihuati is the goodness of oceans and rivers.

Over time, with improvements to marine navigation, humans started to explore the ocean's surface and delineate the contours of the seas. With the gradual acquisition of knowledge, tools, and techniques, humans started to discover properties, nuances, and scientific facts about the sea and also about the seafloor. The exploration of the deep sea and the seafloor are relatively new, and much like the exploration of outer space, generate illusions, human curiosity, and poetical meaning. The nature of the deep ocean was a mystery until the mid-19th century. Inventions like sonar and later the use of manned deep-diving submersibles helped to clarify the nature of such areas. In 1521, 
Ferdinand Magellan dropped a 700 meter long rope from his ship, which did not reach the bottom, and so he concluded that the sea was of infinite depth. Nowadays with the help of multibeam echo sounders we have bathymetries with resolutions of centimeters and a majority of the ocean has been scanned. Marine geology and oceanography are now stable and mature fields of study. Several universities around the world have groups that study the sea and the seafloor. The attention, support, and contributions of such groups will grow in the coming years now that we see more clearly the importance of the sea for the stability of the entire living system, including humans.

On the artistic side, the sea has been a rich source of inspiration over time. Paintings, sculptures, and sound compositions have been created with the sea as the main character. We could mention as musical examples La Mer by Debussy, completed in 1905 after the composer spent several days camping in solitude in front of the sea; Toward the Sea by Toru Takemistu premiered in 1981 as one of several works on the subject of water. As visual works we could mention Sea Life, a woven tapestry created in 1972 by Alexander Calder; and several works of Gauguin and Monet. The list of art that has the sea as topic is so long that it is out of the scope of this work.

\section{E.2 Marine Geology}

\section{E.2.1 Oceanography}

Oceanography is a huge field where many sciences join forces with the goal of understanding the oceans. Geology, geography, geophysics, physics, chemistry, geochemistry, mathematics, meteorology, botany, and zoology play key roles in expanding the knowledge of oceans. Oceanography is such a vast field of study that usually it is broken down into subdisciplines, producing a highly interdisciplinary practice.

Marine geology, for example, includes the study of Earth at the sea's edge and below its surface. Started as an empirical exploration as soon as humans first stood on the shore, it is gradually becoming a formal science. Commercial shipping in the eighteenth century required better charts and more accurate navigation techniques, leading to the first books on oceanography. Formal ocean science began with the expeditions of the nineteenth century and the research of Darwin, Forbes, Mülcer, and others. In the twentieth century, the largest push came from the needs of the military during World 


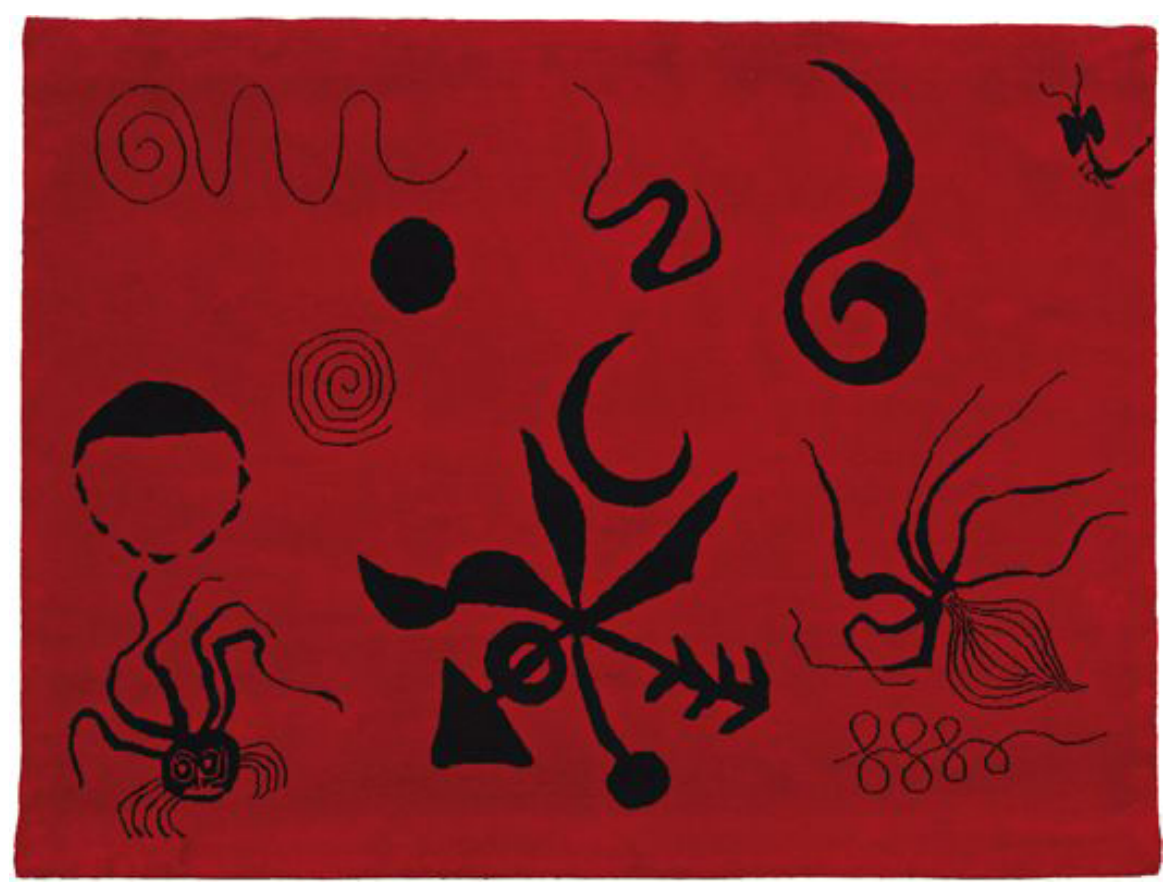

Figure E.2: Alexander Calder, Sea Life (1972).

War II. Nowadays, academic and industrial research play a fundamental role in the study of the sea, focused on global studies and the management of resources. We now know the key role of oceans in the stability of all ecosystems and global stability, therefore its understanding is of extreme importance for human beings.

\section{E.2.2 Earth Origins}

There are many theories about the origins of the planet and the oceans but current evidence suggests the following path as the most accepted version. The theory of the Big Bang states that roughly 13.7 billion year ago a concentrated, extremely hot spot containing all the energy and matter in the universe exploded, causing the universe to expand. In a few seconds the temperature cooled down so light and other forms of radiation could be converted into atoms. Small concentrations of matter began to grow gravitationally, increasing density further and further. About 200 million years after the Big Bang, gravity began to pull matter into the structures we see today, and the first 
stars were formed.

Stars are responsible for the formation of heavy elements by fusing lighter elements in their interiors. While some stars are burning out or exploding, others are still forming with original matter from the Big Bang and recycling matter from older generations of stars. Present theories state that 5 billion years ago our solar system was formed by the collapse of a rotating interstellar cloud of gas and dust, the product of old stars' explosions. A nearby exploding star is thought to have imparted spin to the cloud, generating heat by compression. At the center the sun was formed. Self-sustaining nuclear reactions kept the sun hot but the outer region cooled down and aggregated into structures that formed the planets.

Earth is thought to have been formed from cold matter but it was bombarded by particles that gradually heated it at the same time that layers and layers of elements compressed its interior, heating it even more. A few hundred million years after Earth formed, the interior reached the melting point of heavy metals, $2000^{\circ}$, and the less dense material moved upwards, cooling and solidifying. As Earth warmed and melted, water, hydrogen, and oxygen locked in the minerals ere released and brought to the surface in volcanic eruptions. As Earth's surface cooled, water vapor condensed to form the oceans. Another possible source of water for the ocean is from space objects, such as comet balls or ice meteorites. Oxygen accumulated in the atmosphere until its production exceeded its loss by chemical reactions with the crust. This did not happen until life evolved to a level in which photosynthetic organisms could convert carbon dioxide and water into organic matter and free oxygen. As Earth cooled, the Sun's energy replaced the heat of planet formation in maintaining its surface temperature. The rotation of the Earth, its solar orbit, and its atmospheric gases produce surface temperatures that allow the existence of liquid water.

Today, oceans cover 362 million $\mathrm{km}^{2}$, which represents $71 \%$ of Earth's surface. The volume of water in the oceans is 1.35 billion $\mathrm{km}^{3}$, making it the reservoir for $97.26 \%$ of the water on Earth. The distribution and shape of the continents delineate the Pacific, Atlantic, and Indian oceans. The Arctic Ocean is located at the North Pole and some people count the Southern Ocean as a fifth ocean. 


\section{E.2.3 Earth's Structure and Composition}

After years of research, scientists now know much about the Earth's composition. At the center of the planet is the inner core with a radius of $1222 \mathrm{~km}$. It is composed mainly of iron and is extremely dense due to high pressures. The temperature ranges from $4000^{\circ} \mathrm{C}$ to $5500^{\circ} \mathrm{C}$. The inner core is surrounded by a shell called the outer core, $2258 \mathrm{~km}$ thick, made also of iron but at a lower temperature of about $3200^{\circ} \mathrm{C}$. The outer core is liquid and therefore exhibits fluid dynamics. Outside the outer core we have the mantle, which makes up about $70 \%$ of Earth's volume. The mantle is $2866 \mathrm{~km}$ thick, less dense, and cooler $\left(1100^{\circ} \mathrm{C} 3200^{\circ} \mathrm{C}\right)$ than the core. It is made of magnesium-iron silicates. The mantle is solid but some areas are weaker than others. It is divided in two sublayers: the asthenosphere on the inside which is weak and has ductile behaviors, and the lithosphere on the outside which is strong and has rigid behaviors. In oceanic regions, the thickness of the lithosphere thickens with increasing age of the seafloor, reaching a thickness of about $100 \mathrm{~km}$ at an age of 80 million years.

The exterior of the Earth is a cooler and more rigid layer called the crust. The boundary between the crust and the mantle is a chemical boundary known as the Moho. There are two kinds of crust: continental and oceanic. The continental crust has an average thickness of about $40 \mathrm{~km}$ and is composed primarily of granite-type rock. Oceanic crust is denser than continental crust, has an average thickness of about $7 \mathrm{~km}$, and is composed primarily of basalt-type rock.

\section{E.2.4 Movement of the Continents and Plate Tectonics}

After more than 400 years of speculation and theories, there is now a strong theory supported by experimental data that explains the movement of the continents. Between 1947 and 1965, geologists Bruce Heezen and Marie Tharp systematically mapped Earth's seafloor features, producing the first comprehensive study of seafloor bathymetry. The map revealed the presence of a series of ridges, rises, and trenches. Of high interest were the deep sea's mid-ocean ridge and rise systems and the steep-sided trenches of the Pacific Ocean.

In the early 1960s, H. H. Hess suggested the existence of patterns of moving mantle 


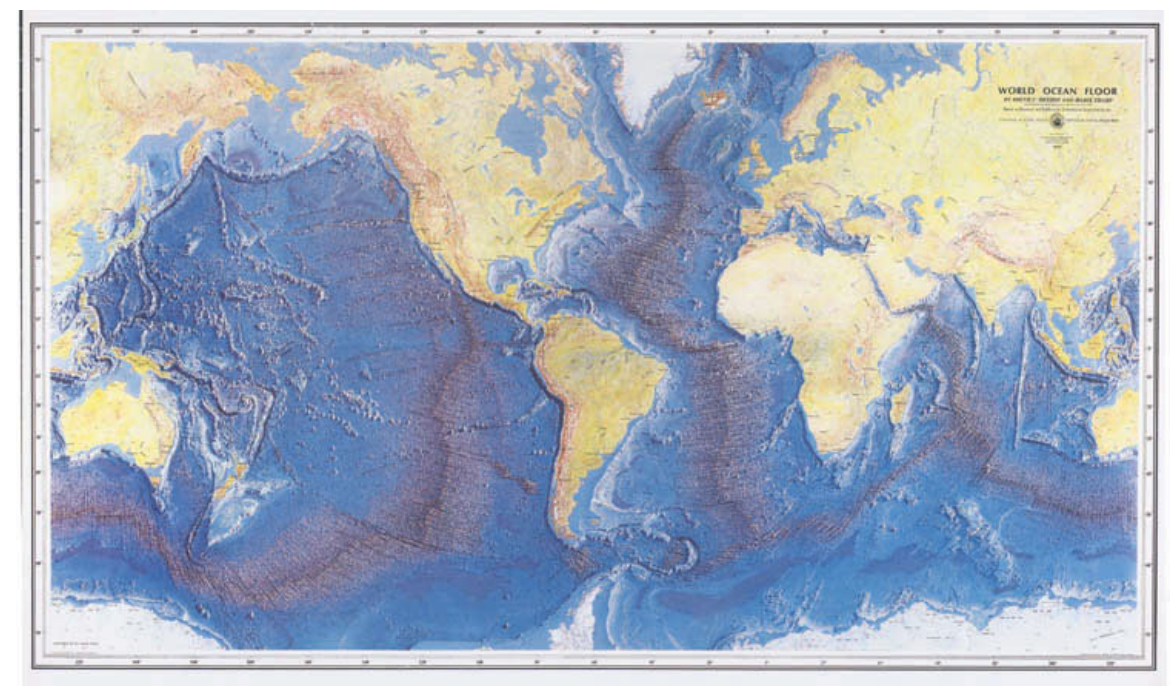

Figure E.3: First physiographic chart of the world's oceans, published in 1974

material called convection cells. According to this theory, there are currents of lowdensity molten material heated by Earth's radioactivity. These currents move mantle rocks upward, producing a horizontal displacement beneath the lithosphere, cooling and increasing in density. At some point, the mantle rock is cool and dense enough to sink once again. The theory explains the creation of the mid-ocean ridge, the creation of new seafloor and oceanic crust, and how old crust descends back into Earth's interior at the deep ocean trenches. The process would produce seafloor spreading, spreading centers, and subduction zones. Seafloor spreading is the force causing movement of the lithosphere, therefore the movement of the continents.

Later data gathered by oceanographers, geologists, and geophysicists validated Hess's theory. New technologies for earthquake measurement and the use of sound waves, the radiometric dating of rocks at different depths and regions obtained by drilling, and finally research in paleomagnetism proved the majority of Hess's hypothesis.

According to the plate tectonics theory, the fragmentation and movement of Earth's outer rigid shell produced the drifting of continents and the motion of the seafloor. The lithosphere is fragmented into seven major plates and a number of smaller ones. Some of them are capped by oceanic, continental, or mixed crust. The plates move with respect 


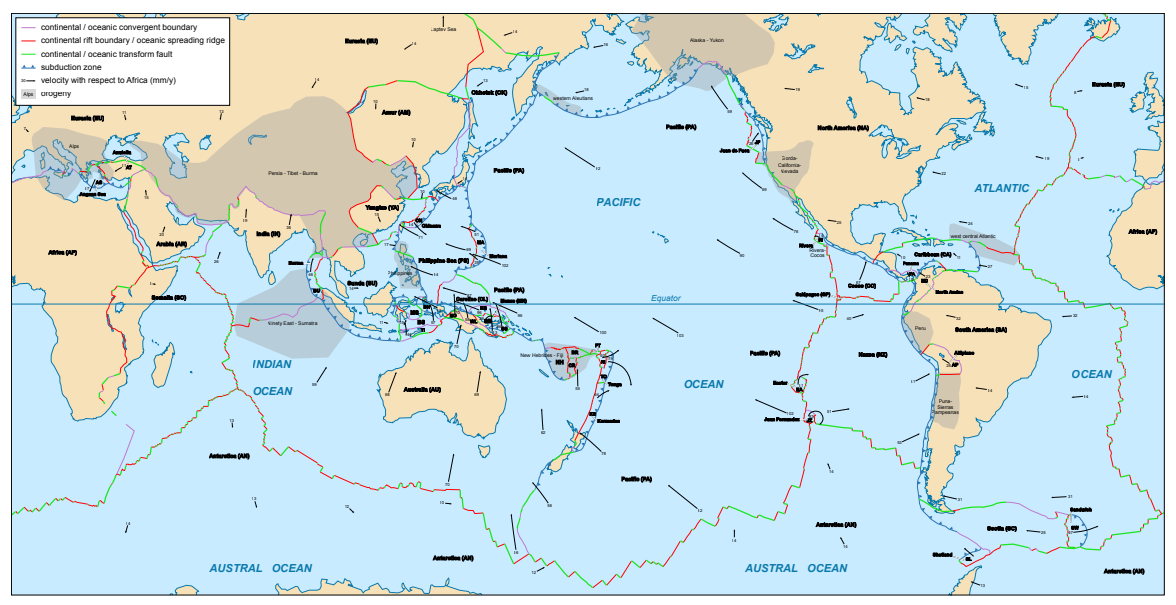

Figure E.4: Earth's lithosphere plates.

to others on the asthenosphere below. As the plates move they interact with one another along their boundaries. There are three basic kinds of plate boundaries, each one associated with different kinds of geological processes. A divergent plate boundary occurs where plates move away from each other, producing ocean basins and new seafloor. Convergent plate boundaries occurs where plates move towards one another, producing deep-ocean trenches, destruction of seafloor, and the creation of massive mountain ranges. Transform boundaries occur where one plate slides past another, making large faults.

\section{E.2.5 Hydrothermal Vents}

The FAMOUS project in 1973 was the first project that explored the rift valley of the mid-ocean ridge. One year before, plumes of hot water were discovered in the Galápagos Rift. The mix of hot water with cold water produced shimmering, upwardflowing streams rich in silica, barium, lithium, manganese, hydrogen sulfide, and sulfur. Surrounding areas were covered with chemical deposits rich in metals precipitated out of the vent waters. In 1979, near the tip of Baja California, at a depth of 2000 meters, oceanographers discovered mounds and chimney-shaped vents 20 meters high ejecting $350^{\circ} \mathrm{C}$ hot water and black streams of particles containing sulfides of lead, cobalt, zinc, 


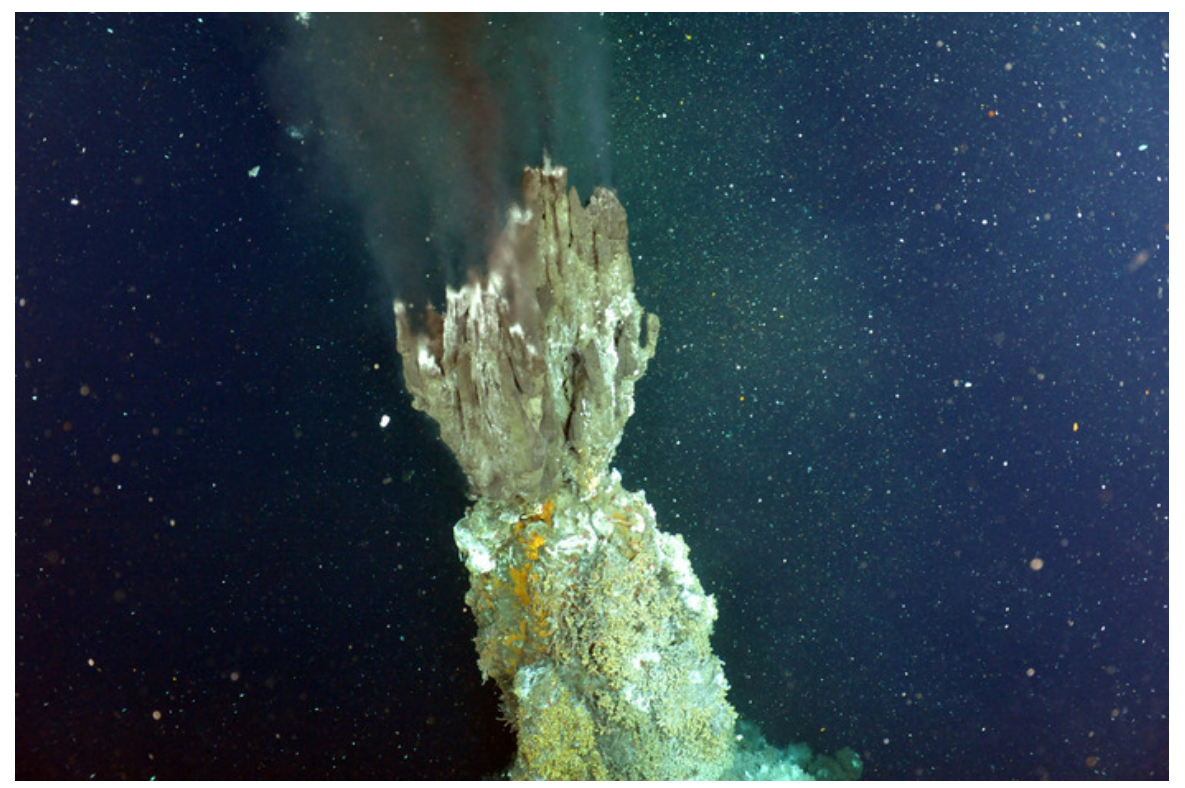

Figure E.5: El Guapo hydrothermal vent.

silver, and other minerals.

It is now known that hydrothermal vent activity occur worldwide at the seafloor. Cold, dense seawater circulates near magma chambers close to the seafloor. It is heated and then released through cracks in the newly created upper lithosphere. Some vents produce low-temperature discharges of clear water up to $30^{\circ} \mathrm{C}$, white smokers that reach $300^{\circ} \mathrm{C}$, and black smokers that release jets of black sulfide water at temperatures of up to $400{ }^{\circ} \mathrm{C}$. The activity of a hydrothermal vent goes on for years, with rapid and unpredictable temperature fluctuations on a timescale of days or seconds, indicating unstable environments.

In the 1970s, the presence of large communities of animals were discovered at hydrothermal vents. Clams, mussels, limpets, tube worms, and crabs live and depend on bacteria that rely on the hydrogen sulfide and particulate sulfur in the hot vent water. No sunlight is necessary in these chemosynthetic communities and no food is needed from the surface. Since the initial discoveries along the Galápagos Rift, communities of vent organisms have been discovered in several rifts worldwide.

Although common hydrothermal vents are located along the axes of ocean ridges 
on young seafloor, there is growing evidence of hydrothermal systems with different chemical signatures located on older seafloor. The discovery and investigation of a hydrothermal vent field named Lost City, located at the axis of the Mid-Atlantic Ridge, is the strongest evidence of such types of systems and features cold water, abnormal alkalinity, and chimneys composed of carbonate and magnesium-rich minerals up to 60 meters tall.

\section{E.2.6 Bathymetry of the Seafloor}

In 85 B.C., the Greek geographer Posidonius, curious about the depth of the ocean, eased a large rock attached to a long rope over the side of a ship in the middle of the Mediterranean Sea. This technique was the method of obtaining soundings for about 2000 years. In the 1920s, acoustic sounding equipment was invented. The echo sounder was used to measure the time required for a sound pulse to leave the ship, reflect at the bottom, and return. Today, there are several techniques for obtaining the depth of the seafloor at several resolutions and producing representations of the depths. Bathymetric measures can be generated by satellite measures, laser airborne depth sounders, and ROVs cameras, among others. The deep seafloor, ranging from 4000 to 6000 meters in depth, covers $30 \%$ of Earth's surface. The deepest known spot in the ocean has a depth of 11,020 meters. Similar to continental crust, the seafloor has an orography made of submarine canyons, abyssal pains, abyssal hills, seamounts, guyots, ridges, rises, and trenches.

\section{E.2.7 Sea Water}

The salt water which comprises the oceans has an average salinity of approximately $35 \%$, but this salinity varies, generally decreasing with latitude and changing in response to the effects of evaporation, precipitation, freezing, thawing, and freshwater runoff from land. The original sources of sea salts include the crust and the interior of Earth. The salt compound is sodium chloride $(N A C l)$, which breaks into positive sodium ions and negative chloride ions. Six ions make up more than $99 \%$ of the salts dissolved in seawater and are known as major constituents. It is known that this proportion in the open ocean is constant with regard to location, salinity, or depth. 


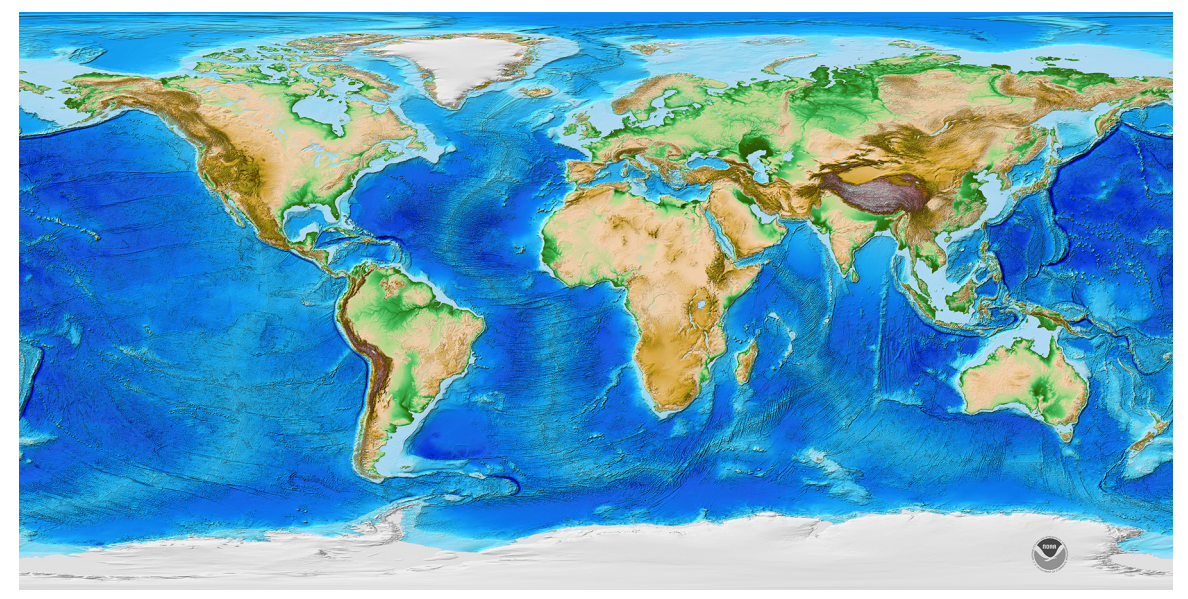

Figure E.6: Bathymetry of the world's ocean basins.

\begin{tabular}{c|c|c|c}
\hline Constituent & Symbol & $\mathrm{g} / \mathrm{kg}$ & Percentage by weight \\
\hline Chloride & $C l^{-}$ & 19.35 & 55.07 \\
Sodium & $\mathrm{Na}^{+}$ & 10.76 & 30.62 \\
Sulfate & $\mathrm{So}_{4}^{2-}$ & 2.71 & 7.72 \\
Magnesium & $\mathrm{Mg}^{2+}$ & 1.29 & 3.68 \\
Calcium & $\mathrm{Ca}^{2+}$ & 0.41 & 1.17 \\
Potassium & $\mathrm{K}^{+}$ & 0.39 & 1.10 \\
\hline
\end{tabular}

Table E.1: Major constituents of seawater without nutrients and dissolved gases. 


\begin{tabular}{l|c|c|c|c}
\hline Gas & Symbol & $\begin{array}{c}\text { Percentage by } \\
\text { volume in } \\
\text { atmosphere }\end{array}$ & $\begin{array}{c}\text { Percentage by } \\
\text { volume in } \\
\text { surface seawater }\end{array}$ & $\begin{array}{c}\text { Percentage by } \\
\text { volume in } \\
\text { total oceans }\end{array}$ \\
\hline Nitrogen & $N_{2}$ & 78.03 & 48 & 11 \\
Oxygen & $\mathrm{O}_{2}$ & 20.99 & 36 & 6 \\
Carbon dioxide & $\mathrm{CO}_{2}$ & 0.03 & 15 & 83 \\
Argon, helium, & $\mathrm{Ar}, \mathrm{He}, \mathrm{Ne}$ & 0.95 & 1 & 100 \\
neon, etc. & & 100.0 & 100 & \\
Totals & & & & \\
\hline
\end{tabular}

Table E.2: Abundance of gases in air and seawater.

It is estimated that the overall salinity of the oceans has remained constant for the last 1.5 billion years, which means that salt ions are removed in some way. The removal of salt ions is through sea spray onto the shore, producing evaporites, and also by precipitation into sediments on the seafloor.

At the sea's surface, gases move between the sea and the atmosphere. The gases dissolve in seawater and are distributed by mixing in different proportions at different depths. The maximum amount of any gas that can be retained in a solution is known as the saturation concentration and depends on the temperature, salinity, and pressure of the water. Therefore the amount of different gases at the ocean varies over regions and depths, producing particular profiles widely used by oceanographers to infer ocean information.

Seawater is slightly alkaline, with a $\mathrm{pH}$ between 7.5 and 8.5 . The $\mathrm{pH}$ of the world's oceans averaged over all depths is approximately 7.8 and remains relatively constant because of the buffering action of carbon dioxide. It is clear that the $\mathrm{pH}$ of seawater strongly depends on the concentration of $\mathrm{CO}_{2}$ in the water which in turns depends on the amount of carbon dioxide in the atmosphere.

Water transmits energy. Seawater transmits electricity because it contains dissolved ionic salts. It also transmits heat, light, and sound. Water is a poor heat conductor by 
conduction; on the other hand, several processes occur through heat convection. Solar radiation also increases the sea temperature, but since it comes from above only a small amount of such energy is slowly transmitted downward by conduction.

Seawater transmits only a small portion of the electromagnetic spectrum. Light energy is attenuated very rapidly by absorption, and scattered by water molecules, ions, and suspended particles. In typical open ocean water, about $50 \%$ of the entering light energy is attenuated in the first 10 meters, and almost all of the light is attenuated at 100 meters. Light in the blue-green to blue-violet range is attenuated less rapidly.

\section{E.2.8 Sound in Seawater}

Sound travels farther and faster in seawater than it does in air; the average velocity of sound in seawater is $1500 \mathrm{~m} / \mathrm{s}$. The speed of sound in seawater is a function of the axial modulus and density of the water:

$$
s=\sqrt{\frac{a m}{d}}
$$

Where $s$ is the speed of sound, am the axial modulus, and $d$ is the density. The axial modulus is a measure of how it compresses. The axial modulus and the density of seawater depends on temperature, salinity, and pressure. The speed of sound increases with increasing temperature, salinity, or depth. Water functions as a low-pass filter so it dissipates the energy of high-frequency sounds.

Since sound is reflected after striking an object, it is has been used for measuring distances. Echo sounders or depth recorders are used to measure the depth of water and such recordings are used to create depth charts. The reflection of sound varies according to the nature of the objects; therefore geologists can detect properties of the seafloor by studying echo charts. Basalt reflects a stronger signal than sediments. The frequency of the sound will also determine the depth of penetration of the sound energy beneath the seafloor. Sound frequencies used by Precision Depth Recorders are generally 5-30kHz. Usually high frequencies are used to measure distance and low frequencies are used to study floor properties since they can penetrate the seafloor sediments and reflect from boundaries between sediment layers. 
At about 1000 meters, the combination of salt content, temperature, and pressure creates a zone of minimum velocity for sound, the SOFAR channel. Sound waves produced in the SOFAR channel do not escape from it unless they are directed outward at a sharp angle and the majority of the sound energy is refracted back and forth along the channel and thus travels great distances. This channel is being used to send sound pulses over long distances to look for long-term changes in the temperature of seawater.

For military purposes, the U.S. Navy installed large arrays of hydrophones on the seafloor. They are now used for acoustic monitoring of marine mammals and seafloor seismic events. Another interesting recent project was the study of pure low-frequency tones of long durations. The sound source was tracked to a region of seamounts at shallow depths. It appears that erupting seamounts produce clouds of bubbles that act as a resonating chamber, and this allows sound waves to oscillate vertically between the volcanic source and the surface of the sea, producing the low-frequency tones.

\section{E.3 The Oceans Observatory Initiative}

The vision of the Oceans Observatory Initiative is to "launch a new era of scientific discovery and understanding of the oceans by creating a human telepresence within the ocean".[40] It is evident that oceans are extremely important for the stability and dynamics of the entire Earth, including human life. For this reason, understanding the way in which oceans behave is vital for understanding life on Earth and ultimately for understanding more about our own relationship with the rest of our environment.

The Ocean Observatories Initiative (OOI) will construct a networked infrastructure of science-driven sensor systems to measure the physical, chemical, geological, and biological variables in the ocean and seafloor. Greater knowledge of these variables is vital for improved detection and forecasting of environmental changes and their effects on biodiversity, coastal ecosystems, and climate.

The ocean is the planet's largest ecosystem. It drives an incredible range of natural phenomena, including our climate, and thus directly impacts human society. New approaches are crucial to bettering our scientific understanding of episodic and long-term changes at work in our oceans. Resolving 


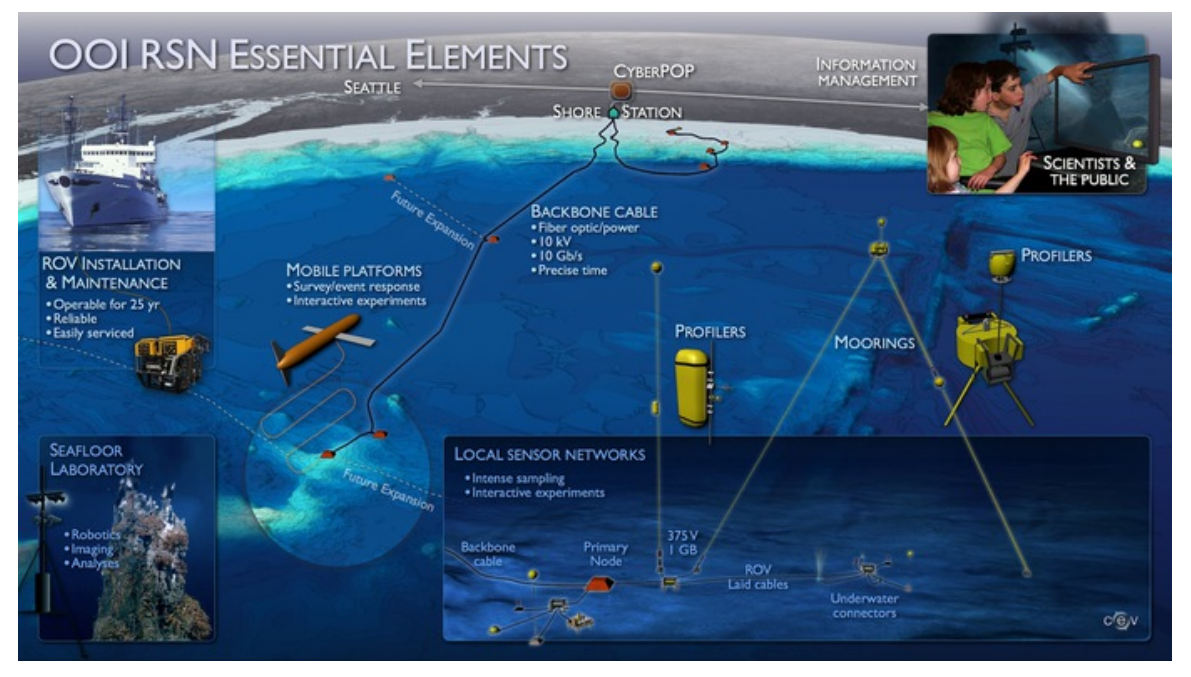

Figure E.7: OOI RSN essential elements. (courtesy of OOI)

pressing issues related to climate variability, severe weather, ocean turbulent mixing, changes in ocean ecosystems, plate tectonics, and sub-seafloor chemistry and biology depend upon these new approaches. The OOI's goal is to install transformational technology in ocean observatories where it can serve researchers, policymakers, and the public.

Building on last century's era of ship-based expeditions, recent technological leaps have brought us to the brink of a sweeping transformation in our approach to ocean research- the focus on expeditionary science is shifting to a permanent presence in the ocean. The ocean itself presents major obstacles to oceanographic exploration. We cannot live in it or even visit for long. We cannot see through it, nor probe effectively with satellites. But new tools permanently installed in our oceans can communicate instantly with scientists on land. They require less power and can carry out user commands or complex pre-programmed instructions; the tools can provide long-term, continuous, and real-time understanding of critical ocean phenomena.

Advanced ocean research and sensor tools represent a significant improvement over past techniques. Remotely operated and autonomous vehicles go deeper and perform longer than submarines. Underwater samplers do in 
minutes what used to take hours in a lab. Telecommunications cables link experiments directly to office computers and supply unparalleled power. Farther asea, satellite uplinks shuttle buoy data at increasing rates.

With these advances the OOI will improve the rate and scale of ocean data collection, and its networked observatories will focus on global, regional, and coastal science questions. They will also provide platforms to support new kinds of instruments and autonomous vehicles.

The major partners in the OOI construction process, three of the four OOI Implementing Organizations (IO), were selected in 2007 by an acquisition process similar to that used in large federal acquisitions. Subawards were established to the University of Washington as the IO for the Regional Scale Nodes, the University of California San Diego (UCSD) as the IO for the Cyberinfrastructure, and the Woods Hole Oceanographic Institution with two consortium partners, Scripps Institution of Oceanography and Oregon State University, as the IO for the Coastal and Global Scale Nodes. The fourth IO, Rutgers, The State University of New Jersey, was selected in 2011 as the IO for the Education and Public Engagement software infrastructure component, with its partners the University of Maine and Raytheon Mission Operations and Services.[30]

As mentioned in the previous quotation, the way oceanography has been studying ocean processes has been limited to short periods of time in comparison with the time span of geological processes. Some of the reasons for this are the complicated logistics involved with long term expeditions, the difficulty of staying on the ocean due to adverse weather conditions, the difficulty of maintaining moorings for long term experiments, and the lack of high power and high bandwidth using solar power technology.

Knowing the limitations of traditional procedures of oceanographic research and knowing the benefits of stable technologies such as fiber optics, the time arrived for proposing a cabled observatory capable of carry electrical power (up to $200 \mathrm{~kW}$ ) and telecommunications bandwidth (up to $240 \mathrm{Gbits} / \mathrm{sec}$ ) into the oceans. This network would power scientific devices such as sensors, cameras, and robots; and would allow 


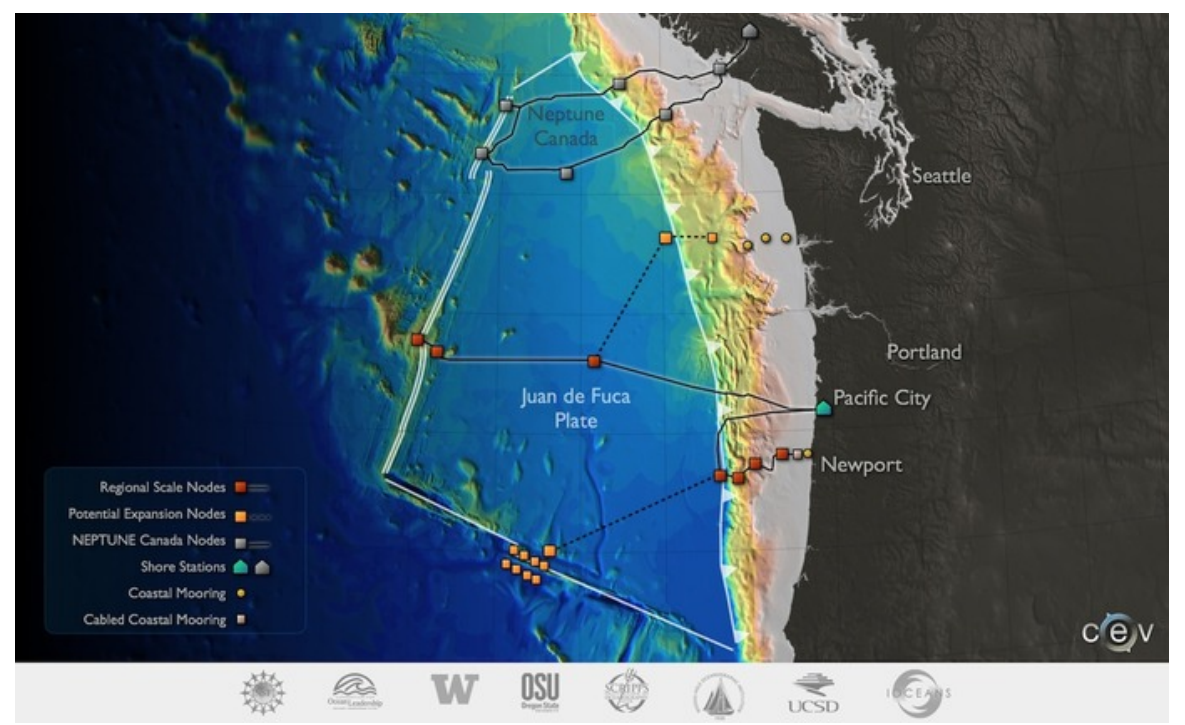

Figure E.8: OOI network plan. (courtesy of OOI)

the continuous transmission of data in real time.

\section{E.3.1 Ocean Observatories Initiative's Regional Scale Nodes}

The University of Washington is leading the design, development, and construction of the NSF Ocean Observatories Initiative's Regional Scale Nodes, the first U.S. regional cabled ocean observatory. This novel cabled ocean observatory will be launched with nearly 900 kilometers of cable across the Juan de Fuca tectonic plate, running from a shore station in Pacific City, Oregon, out to the Juan de Fuca Ridge and south along the Cascadia subduction zone to Hydrate Ridge. Nodes densely populated with instruments and full water column moorings will be installed at study sites at Hydrate Ridge, Axial Volcano, and shallow coastal sites west of Newport Oregon. High bandwidth data and video imagery will be transmitted in real time via the Internet and will be accessible to users around the world: to scientists, educators, students, and decision-makers. The system is designed for a 25 year lifetime. 
The scientific potential and benefits of such an endeavor are evident and will lead to new discoveries and understanding of the ocean. Observing a region of the sea with the continuity and accuracy that the Observatory will provide will certainly provide information and knowledge never available before. Subtle and gradual changes that are lost in the step observations made by ship expeditions will be clearly seen and will offer a set of information that will derive new knowledge that may clarify and explain a set of unknown behaviors of the sea. Knowing more about how the sea works is, in the end, a way to know more about the Earth and more about ourselves as humans.

\section{E.3.2 History}

It is fascinating to observe the evolution of human ideas. As clearly seen along the entire history of humanity, nothing grows from the bloom and all ideas evolved from previous concepts and previous ideas nurtured by developments in other fields of knowledge. Originality relies not on creating something absolutely different but on knowing which previous ideas should be integrated in order to create something that should have been there before. Recording oceanographic data continuously had been done before with much more modest equipment but the OOI puts the logistical, organizational, economical, and technical elements all together.

The concept of a regional cabled ocean observatory -a system that would provide continuous high power and interactive real-time high-bandwidth data transfer to and from shore- began to be seriously explored by scientists at the University of Washington in the mid 1990s.

This vision carried with it solutions to some of oceanography's most difficult challenges: how to study natural phenomena on time scales that range from seconds to decades and on spatial scales from microns to kilometers. The vision provided novel alternatives to science investigations based on limited and uncertain life spans of battery-powered instruments and on the vagaries of northeast Pacific storms that restrict ship-based expeditions to field seasons of only a few months each year.

Exciting exchanges between scientists and engineers led to a plan for a 
network of distributed sensors in the oceans attached to submarine fiberoptic/power cables connected to land. The fiber would provide continuous power to interactive, networked instrument arrays that would capture data and immediately send it to shore and onto the Internet. Such a system could encircle an entire tectonic plate and the ocean above it.[40]

\section{E.3.3 Research Within the OOI}

The Ocean Observatory Initiative develops a variety of research during the expeditions that includes ${ }^{3}$ :

- Methane ice on the seafloor

- Interdisciplinary research on submarine volcanoes

- Cascadia Subduction Zone and large earthquake events

- Climate change

- Oceans and life

- Carbon cycle

- Ocean acidification

- Societal implications

\section{E.3.4 Technology at the OOI}

In order to achieve the project's goals, the expedition requires and uses a set of cuttingedge technologies for measuring, sensing, locating, plotting, and analyzing oceanographic information. Some of these technologies are presented on the following list $^{4}$ :

\footnotetext{
${ }^{3}$ This information has been extracted from Interactive Oceans[40].

${ }^{4}$ This information and the description has been copied from Interactive Oceans [40].
} 
RESON Sonar The RESON SeaBat 7125 multibeam sonar aboard both the AUV Sentry and ROV Jason enables scientists to collect extremely detailed 3-D representations of underwater features and seafloor conditions.

Chirp Sonar A Chirp sub-bottom profiler aboard the AUV Sentry will aid in seafloor surveys of methane seeps at the summit of the Southern Hydrate Ridge. This equipment helps to classify the distribution of gas hydrate within the study area because a distinctive acoustic reflection known as the bottom-simulating reflector occurs at the boundary of zones that have solid gas hydrates.

HD Imagery High-definition video imagery from the seafloor is collected using an Insite Pacific underwater video camera. The camera is mounted on ROV Jason and images are transmitted to the ship via fiber-optic cable.

ROV Jason The remotely operated vehicle (ROV) Jason 2 is a robotic vehicle tethered to the ship by a fiber optic cable that is capable of working in water depths up to 6500 meters. Jason is designed to conduct a diverse range of scientific investigations in the world's oceans. It is operated by the Woods Hole Oceanographic Institution and is part of the National Deep Submergence Facility.

AUV Sentry Sentry is an autonomous underwater vehicle (AUV), an underwater robot that swims freely beneath the surface without being tethered to a ship, in water depths up to 4500 meters. During the cruise, Sentry was preprogrammed to "fly" over the node sites along overlapping survey lines at a constant altitude above the seafloor. Sentry is operated by the Woods Hole Oceanographic Institution and is part of the National Deep Submergence Facility.

Gravity Core Gravity cores enable scientists to sample and study sediments from the seafloor reliably and at relatively little cost. During this cruise, we will use gravity cores to help identify the best locations for the RSN nodes.

EM 300 Sonar The EM300 system is mounted on the hull of the R/V Thomas G. Thompson. This multibeam echosounder sends out multiple waves of sound (pings 
of $30 \mathrm{kHz}$ acoustic energy) that cover a distance on the seafloor that is roughly equal to two times the water depth. The sound signals that bounce off the seafloor are received back at the ship at different times. Computers on the ship process this information to determine water depth, which is then plotted as a bathymetric map. On this cruise, we expect the EM300 to generate maps at 50-meter resolution. Data collected from EM300 surveys are used to generate base maps that cover more extensive areas of the seafloor that we can then use for planning higher resolution near-bottom surveys with vehicles like Sentry and Jason.

\section{E.3.5 Expeditions of the OOI}

In order to achieve such a long-term and ambitious project, the goals had to be segmented into several sequential steps. Each step contains an expedition that works for several proposes. Each expedition has a main research goal, an educational section, and a set of secondary research objectives that complement the main objective. Through 2013, seven expeditions have been organized by the University of Washington:

Visions 2005 At this point project NEPTUNE was still not part of the Ocean Observatory Initiative but the cabling observatory was already a central part of the expedition. The major components of the expedition were exploring the limits of life in hydrothermal sulfide deposits by drilling holes and inserting an instrument known as the microbial incubator; documenting the linkages among deformation, venting behavior, fluid chemistry, and microbial activity along the boundaries of tectonic plates; conducting a program focused on professional development for $\mathrm{K}$ 12 science teachers; conducting a cable route survey for the northern portion of the NEPTUNE network; and, transmitting high-definition video imagery live from the seafloor and the ship via satellite to shore.

Insite 2008 The primary purpose of this cruise was to map two regions of the seafloor in order to determine the best location for setting the primary nodes of the Regional Scale Nodes. One area is near Hydrate Ridge, just off the central Oregon coast due west of Newport, Oregon, and the other area of interest is near Axial Volcano, about 250 miles west of Newport. The mapping was obtained with the 
EM300 multibeam sonar mounted on the hull of the vessel, with the Autonomous Underwater Vehicle Sentry, and also with a deep-towed digital camera, TowCam mounted on a ROV.

Lumens 2009 The cruise was scheduled from August 1 to August 15 with the major goal of mapping node sites from Hydrate Ridge east towards Newport Oregon, and west of Grays Harbor, Washington. The expedition got only as far as the Ballard Locks before the $\mathrm{R} / \mathrm{V}$ Thompson experienced serious mechanical problems and the cruise was cancelled. The Axial project was initially planned to be realized on this cruise.

Enlighten 2010 The primary purpose of the cruise was to lay the foundation for the installation of secondary infrastructure (nodes, junction boxes, sensors, moorings, and extension cables) on the Regional Scale Nodes (RSN) cabled ocean observatory. The Axial project was realized during this expedition while water was sampled and analyzed at depths of 500 to 2500 meters; sediments and gas hydrates were sampled in order to understand the seafloor's composition, "strength," and coarseness; high-resolution mapping systems were employed to produce a meterscale resolution bathymetric map in order to produce a site characterization for mooring west of Grays Harbor; Sentry and Jason were used to produce a one-meter resolution bathymetric map of the Southern Hydrate Ridge, one of the most well studied gas hydrate systems; and RESON was used to map the most robust volcano on the Juan de Fuca Ridge, the Axial Seamount.

Visions 2011 The major goals for the expedition were conduct deployment and recovery tests of the RSN primary nodes by L3-MariPro; complete site verification of secondary node and sensor locations at Southern Hydrate Ridge and Axial Seamount; conduct mapping and physical properties studies of the $10 \mathrm{~km}$ extension cable route from primary node 1B to the Southern Hydrate Ridge; complete site verification for full water column mooring infrastructure at the base of the Axial Seamount; and, conduct surveys of the backbone cable deployed at Axial Seamount by L3-MariPro prior to this cruise. The Axial exhibition was produced 


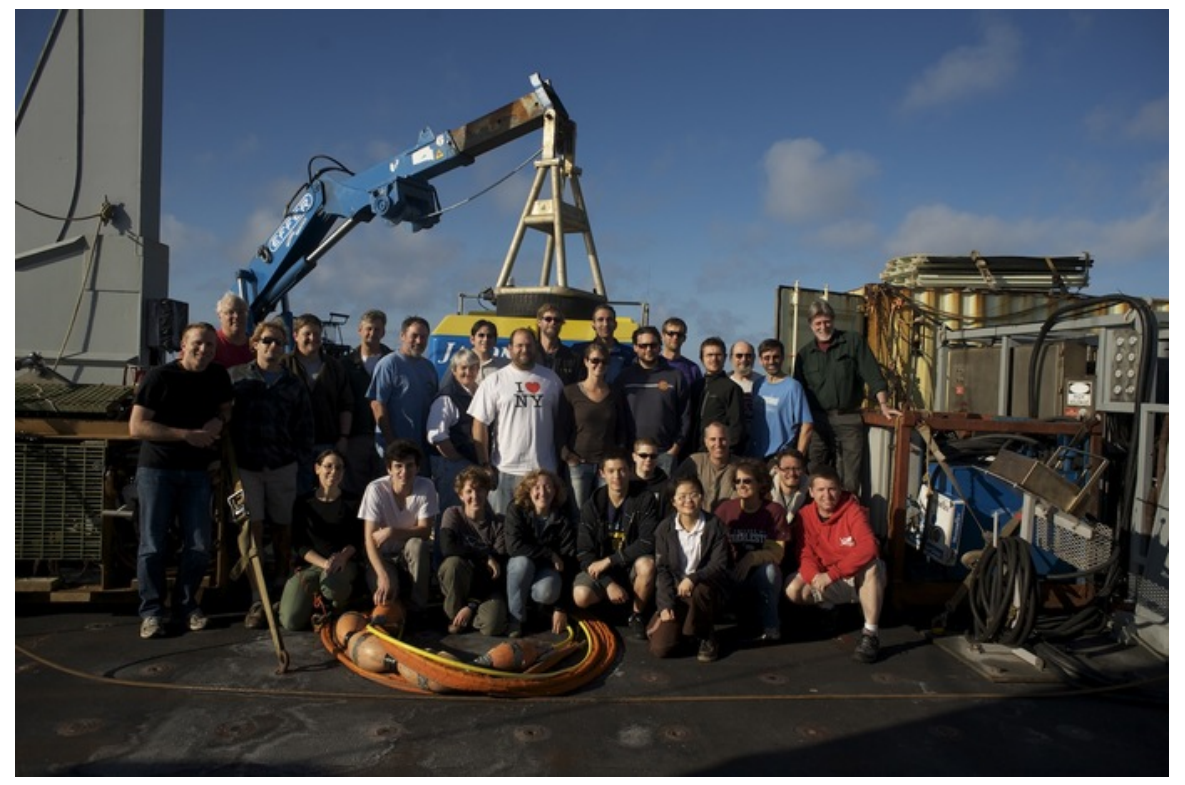

Figure E.9: Enlighten Leg 2 Science Party (courtesy of OOI).

immediately prior to this cruise.

Visions 2012 The primary purpose of this expedition was to continue surveys and assessments related to the installation of the OOI's high-power and high-bandwidth cabled ocean observatory. The expedition was canceled due to necessary repairs on the $\mathrm{R} / \mathrm{V}$ Thompson.

Visions 2013 This expedition is underway at the time of writing. It is focused on deploying and testing nearly 24,000 meters of extension cables that are part of the secondary infrastructure. Some of the cable had been recently deployed by the contractor L-3 MariPro. A set of sensors is also being installed. 


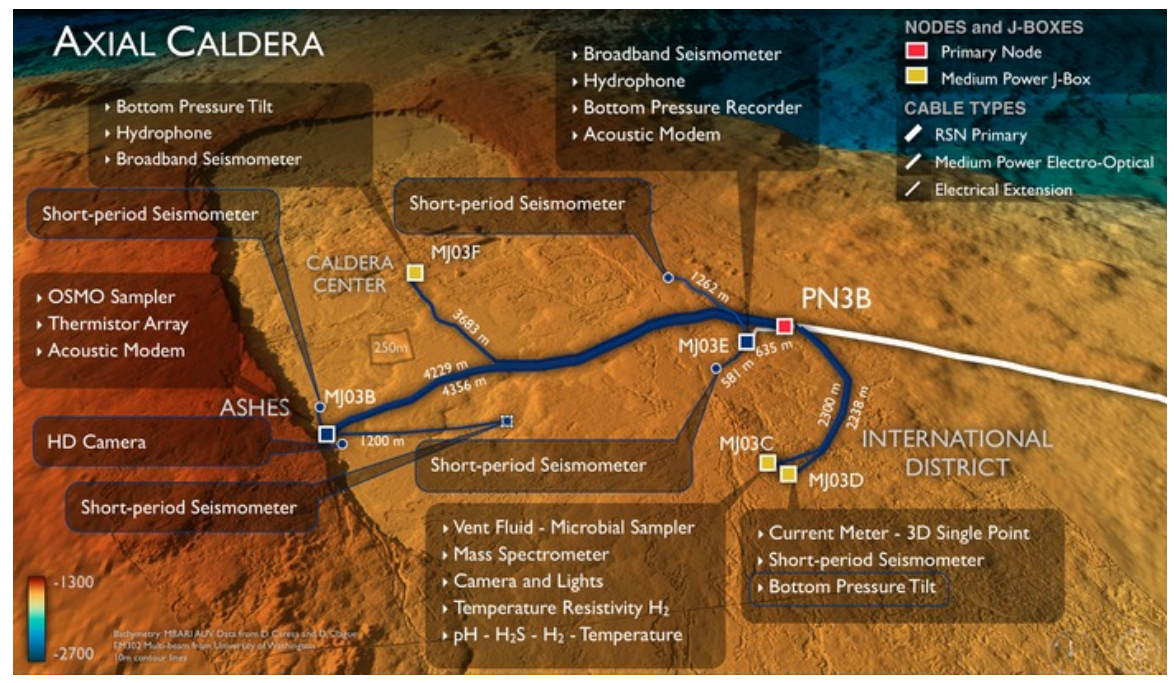

Figure E.10: Axial Caldera infrastructure (courtesy of OOI). 


\section{BIBLIOGRAPHY}

[1] BitCloud Developers Guide. ATMEL.

[2] BitCloud Quick Start Guide. ATMEL.

[3] Francesco Careri. Walking as an aesthetic practice. Land\&Scape. Gustavo Gili, SL, 2007.

[4] Joel Chadabe. Electric Sound: The Past and Promise of Electronic Music. Prentice-Hall, 1997.

[5] Nick Collins and Julio d'Escrivan, eds. The Cambridge Companion to Electronic Music. Cambridge University Press, 2007.

[6] Richard Colson. The Fundamentals of Digital Art. AVA, 2007.

[7] Christoph Cox and Daniel Warner, eds. Audio Culture; readings in modern music. Continuum, 2007.

[8] Timothy J. Crone et al. "The Sound Generated by Mid-Ocean Ridge Black Smoker Hydrothermal Vents". In: PLoS ONE 1 (2006).

[9] Steve Dixon. Digital Performance; A History of New Media in Theater, Dance, Performance Art, and Installation. MIT press, 2007.

[10] Umberto Eco. La Obra abierta. Plantea De-Agostini, 1992.

[11] Anne Focke, ed. Trimpin; Contraptions for Art and Sound. University of Washington Press, 2010.

[12] Hans-Georg Gadamer. La actualidad de lo bello; el arte como juego, símbolo y fiesta. Ediciones Paidós, 1977.

[13] Kyle Gann. The music of Conlon Nancarrow. Cambridge University Press, 1995.

[14] Maria Anna Harley. "Space and Spatialization in Contemporary Music; History and Analysis, Ideas and Implementations". PhD thesis. McGill University, Faculty of Music, 1994. 
[15] Bruce Hemingway, Waylon Brunette, and Gaetano Borriello. "Variations on the Flock: Wireless Embedded Systems in an Undergraduate Curriculum". In: Australian Telecommunications, Networks and Applications Conference. Dec. 2006, pp. $366-370$.

[16] Bruce Hemingway et al. "The Flock: Mote Sensors Sing in Undergraduate Curriculum". In: vol. 37. 8. IEEE Computer. 2004, pp. 72-78.

[17] Douglas Kahn. Noise Water Meat; A history of sound in the arts. MIT press, 2001.

[18] Gary Kendall. "A 3-D Sound Primer: Directional Hearing and Stereo Reproduction". In: Computer Music Journal 19.4 (1994).

[19] Gary Kendall. "The Decorrelation of Audio Signals and Its Impact on Spatial Imagery". In: Computer Music Journal 19.4 (1995).

[20] Seth Kim-Cohen. In the Blink of an Ear; Toward a Non-Cochlear Sonic Art. 1st. Bloomsbury Publishing, 2009.

[21] Dierk König et al. Groovy in Action. 2nd. Manning Publications, 2009.

[22] Berlin Akademie der Kunste. Klangkunst. Prestel-Verlag, 1996.

[23] Brandon LaBelle. Acoustic Territories; sound culture and everyday live. Continuum, 2010.

[24] Brandon LaBelle. Background Noise; Perspectives on Sound Art. Continuum, 2007.

[25] Michael Lailach. Land Art. Ed. by Uta Grosenick. Taschen, 2007.

[26] Alan Licht. Sound Art; Beyond Music, Between Categories. Rizzoli International Publications. Inc., 2007.

[27] Rubén López-Cano. Música Plurifocal; Conciertos de Ciudades de Llorenç Barber. Euterpe. Biblioteca Litterarum Humaniorum, 1997.

[28] Mega 168 Datasheet. ATMEL.

[29] Paul D. Miller, ed. Sound Unbound; Sampling Digital Music and Culture. MIT press, 2008. 
[30] Ocean Observatories Initiative Web. July 2013. URL: http : / / www . oceanobservatories.org (visited on $07 / 23 / 2013$ ).

[31] Christiane Paul. Digital Art. 2nd. World of art. Thames and Hudson, 2008.

[32] G. Proskurowski et al. "Ocean Observing at Axial Seamount: Details of the Current OOI-RSN Design Within the Context of the April 2011 Eruption". In: AGU Fall Meeting Abstracts (Dec. 2011).

[33] Bernd Schulz, ed. Resonances. Kehrer, 2002.

[34] SerialNet User Guide. ATMEL.

[35] Edward A. Shanken, ed. Art and Electronic Media. Phaidon, 2009.

[36] Hugo Solís. El espacio físico como variable estructural en música. Tech. rep. Escuela Nacional de Música and Instituto de Investigaciones Estéticas, UNAM, 1999.

[37] Hugo Solís. "GAB: Sistema de reinterpretación para pianos". MA thesis. Escuela Nacional de Música UNAM, Nov. 2001.

[38] Keith A. Sverdrup and Ginger (Virginia) Armbrust. An introduction to the worlds oceans. McGraw-Hill, 2008.

[39] Bruce Wands. Art of the Digital Age. Thames and Hudson, 2006.

[40] University of Washington. Interactive Oceans. URL: http : / / www . interactiveoceans, washington. edu (visited on 07/23/2013).

[41] Scott Wilson, David Cottle, and Nick Collins, eds. The SuperCollider Book. MIT Press, 2011.

[42] Stephen Wilson. Information Arts; intersections of art, science, and technology. MIT Press, 2002.

[43] Trevor Wishart. On Sonic Art. Contemporary Music Studies. Taylor and Francis, 1996.

[44] ZigBee Specification Document 05347r17. Tech. rep. ZigBee Standards Organization, Jan. 2008.

[45] ZigBit 2.4 GHz Amplified Wireless Modules Datasheet. ATMEL. 


\section{VITA}

Hugo Solís García was born in Mexico City in 1976. He holds a Bachelors degree in Piano performance from the Escuela Nacional de Música of the Universidad Nacional Autónoma de México; a Masters degree in Media Arts and Sciences from the Media Laboratory of the Massachusetts Institute of Technology; and a Diploma de Estudios Avanzados of Computer Sciences and Digital Communication from the Music Technology Group of the Pompeu Fabra University.

He has received grants, prizes, and awards from SEP, FONCA, UNAM, TELMEX, MIT, UW, IMEB, 4Culture, and the City of Seattle among others. His work is shown and performed regularly in festivals and events internationally.

He is currently Professor of Computer Science at the Instituto Tecnológico de Estudios Superiores de Monterrey campus Ciudad de México where he teaches courses in Digital Sound and conducts research in the field of Sound and Electronic Art. Hugo is member of the Sistema Nacional de Creadores de Arte of Mexico. 


\section{COLOPHON}

This document was developed using the iOS operating system. The text was produced with $\mathrm{AT}_{\mathrm{E}} \mathrm{X} 2_{\varepsilon}$. The $\mathrm{ATT}_{\mathrm{E}} \mathrm{X} 2_{\varepsilon}$ template was derived from the University of Washington template of Jim Fox. $\mathrm{HT}_{\mathrm{E}} \mathrm{X} 2_{\varepsilon}$ was manipulated with TeXSHop. References were organized and generated with BibTex. Most images were edited at some point with Adobe Photoshop. Diagrams were produced with Xmind Pro.

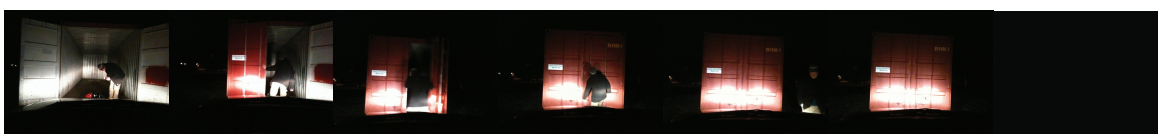

